\title{
A Study of Muon Ionization Cooling at MICE
}

\author{
A DISSERTATION \\ SUBMITTED IN PARTIAL FULFILLMENT OF \\ THE REQUIREMENTS FOR THE DEGREE OF \\ DOCTOR OF PHILOSOPHY
}

Department of Physics, Graduate School of Science Osaka University

by

Hideyuki Sakamoto

February, 2010 
Abstract : An Neutrino Factory based on a high-energy muon storage-ring is proposed to study neutrino oscillation with high precision. An emittance reduction of muon beam by ionization cooling, which has never been demonstrated in practice, is one of the critical issues for Neutrino Factory. The international Muon Ionisation Cooling Experiment (MICE) is the first experiment to verify an effect of the ionization cooling with muons. MICE will measure a change in transverse emittance of approximately $10 \%$ with a precision of $\pm 0.1 \%$.

In order to meet the requirements, muon trackers based on $350 \mu \mathrm{m}$ diameter scintillating fibers have been proposed. The construction of such trackers is a very challenging task and some innovative techniques are needed to realize, since there have been no trackers made with such a small diameter of scintillating fibers in the world. Upstream and downstream SciFi trackers have been successfully constructed with the international collaboration of UK, US and Japan by 2008. Both of the trackers have been tested with cosmic-rays at the RAL by 2009 , at which high tracking efficiencies more than $90 \%$ are measured for both trackers. It is also confirmed that by collecting the misalignments found in both of the trackers, the requirements for the emittance measurement is met. 


\section{Contents}

1 Physics Motivations for Neutrino Factory 1

1.1 Neutrino Oscillation Physics . . . . . . . . . . . . . . . . . . . . . . 1

1.1.1 Neutrino Oscillation . . . . . . . . . . . . . . . . . . . . 1

1.1.2 Forthcoming measurements of the oscillation parameters . . . . . . . . . 2

1.2 Neutrino Factory Concept . . . . . . . . . . . . . . . . . . . 3

1.2.1 Neutrino oscillation measurements at a Neutrino Factory . . . . . . . . . . . 3

1.2.2 High-intensity neutrino deep inelastic scattering . . . . . . . . . . . . . 6

1.2 .3 Non-neutrino science at a Neutrino Factory . . . . . . . . . . . . . . . . 8

1.2.4 Neutrino Factory accelerator complex . . . . . . . . . . . . . . . 8

2 Principle of Ionization Cooling $\quad 11$

2.1 Ionization cooling . . . . . . . . . . . . . . . . . . . . . 11

2.2 Cooling channel design . . . . . . . . . . . . . . . . . . . 12

3 The International Muon Ionization Cooling Experiment (MICE) 15

3.1 Layout of the experiment . . . . . . . . . . . . . . . . 15

3.2 Beam dynamics and quantities to be measured . . . . . . . . . . . . 16

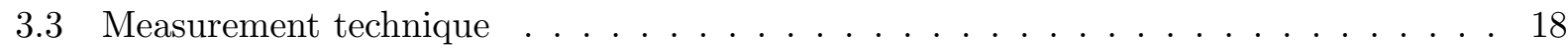

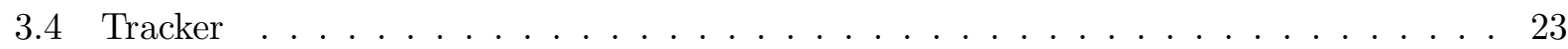

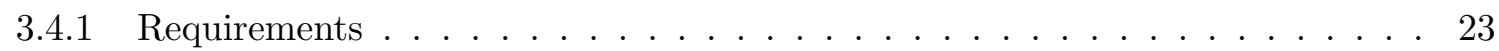

3.4 .2 Layout . . . . . . . . . . . . . . . . . . . . . . 23

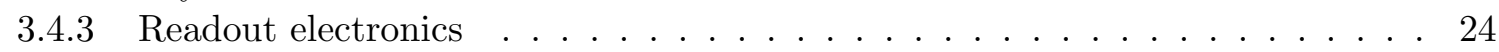

3.4.4 TPG for the Optional Tracker . . . . . . . . . . . . . . . . 24

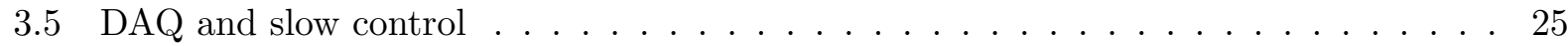

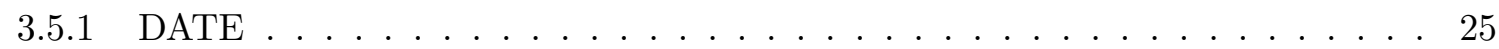

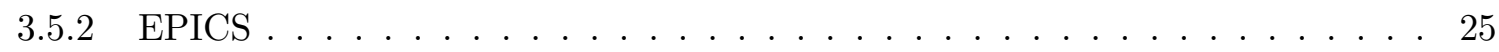

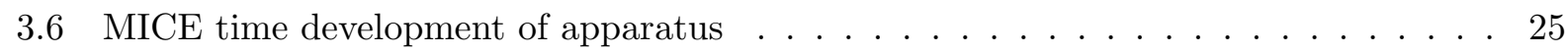

4 Overview of the MICE Scintillating Fiber Tracker $\quad 27$

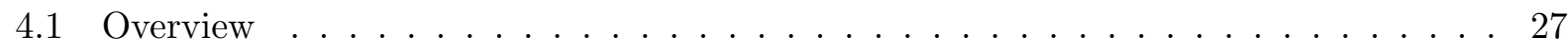

4.1 .1 Requirements . . . . . . . . . . . . . . . . 27

4.1 .2 Key challenges . . . . . . . . . . . . . . . . . 27

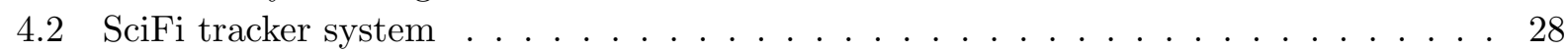

4.2 .1 Layout of the system . . . . . . . . . . . . . . . . . . . . . . . . . . . . . . . . .

4.2 .2 SciFi tracker . . . . . . . . . . . . . . . . . . . 28

4.2 .2 .1 SciFi ribbon . . . . . . . . . . . . . . . 28 
4.2 .2 .2 SciFi station . . . . . . . . . . . . . . . . . . 28

4.2.2.3 Station support frame . . . . . . . . . . . . . . . . 28

4.2 .3 Light-guide . . . . . . . . . . . . . . . . . . . . . . . . 30

4.2.3.1 Internal light-guide . . . . . . . . . . . . . . . . . 30

4.2.3.2 External light-guide . . . . . . . . . . . . . . . . . 32

4.2 .3 .3 Patch panel . . . . . . . . . . . . . . . . 32

4.2 .4 Photon detecting system . . . . . . . . . . . . . . . . . . 32

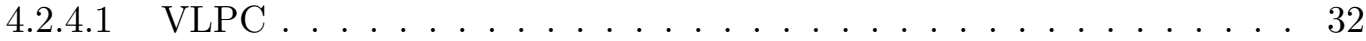

4.2 .4 VLPC cassette . . . . . . . . . . . . . . . . 34

4.2.4.3 VLPC cryostat with cryocooler . . . . . . . . . . . . 35

4.3 Tracker Readout . . . . . . . . . . . . . . . . . . 36

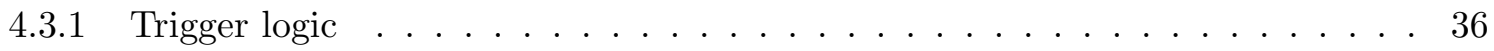

4.3 .2 Electronics . . . . . . . . . . . . . . . . . 37

4.3.2.1 AFEIIt board . . . . . . . . . . . . . . . 37

4.3.2.2 VLSB slave board . . . . . . . . . . . . . . . . . 37

4.3.2.3 DG2020/VLSB master board . . . . . . . . . . . . . . . . . . 38

4.3.2.4 MIL-1553 board . . . . . . . . . . . . . . . 38

4.3 .3 Tracker DAQ . . . . . . . . . . . . . . . . . . 38

4.3 .4 Tracker slow control . . . . . . . . . . . . . . . . . 38

5 SciFi Tracker Prototype $\quad 41$

5.1 Mechanical design for prototype . . . . . . . . . . . . . . . . 41

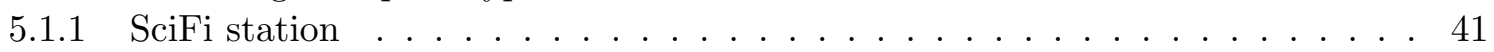

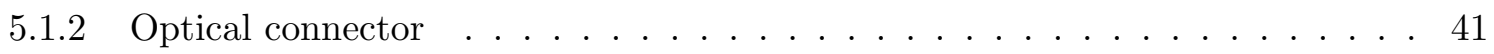

5.1 .3 Scintillating fiber . . . . . . . . . . . . . . . . . . 42

5.1 .4 Light-guide . . . . . . . . . . . . . . . . . . . . 43

5.2 Attenuation length measurement . . . . . . . . . . . . . . . . . 43

5.2 .1 Scintillating fiber . . . . . . . . . . . . . . . . . . . . . . . . . . . . . .

5.2 .2 Clear fiber . . . . . . . . . . . . . . . . . . . . 44

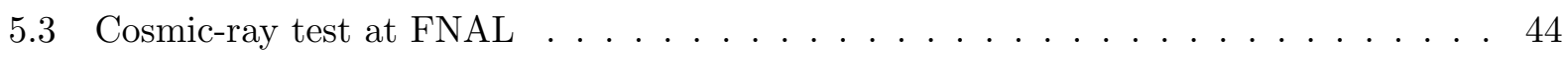

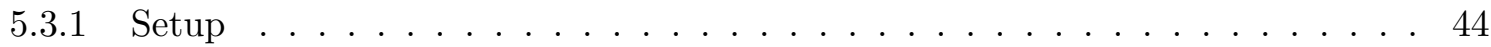

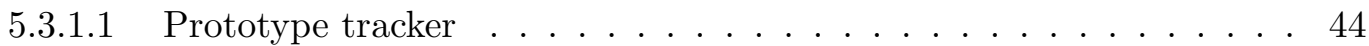

$5.3 .1 .2 \quad$ Light-guide . . . . . . . . . . . . . . . . . . . . . . . . . . . . . . . . . 48

$5.3 .1 .3 \quad$ VLPC cryostat . . . . . . . . . . . . . . . . . 48

5.3 .1 .4 Trigger counter . . . . . . . . . . . . . . . 48

5.3 .2 Read-out . . . . . . . . . . . . . . . . . 48

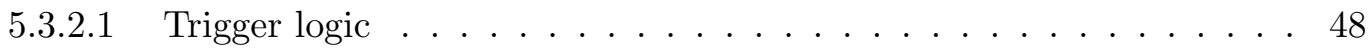

5.3 .2 .2 Electronics . . . . . . . . . . . . . . . . . . . 48

5.3 .2 .3 DAQ and slow control . . . . . . . . . . . . . 48

5.3.3 Light-yield measured with different kinds of concentrations of $3 \mathrm{HF} \ldots \ldots$

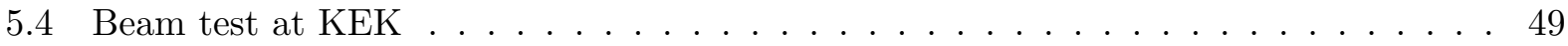

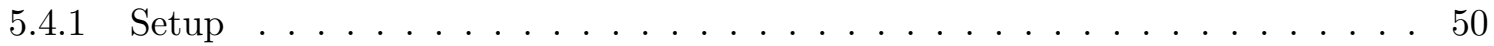

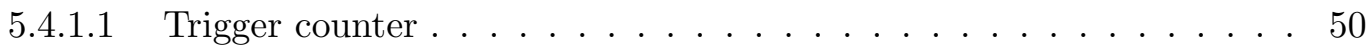

5.4 .1 .2 Cherenvkov counter . . . . . . . . . . . . 50

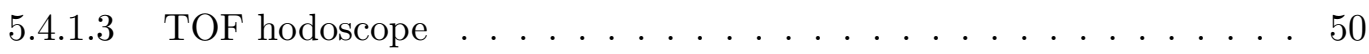

5.4 .1 .4 Beam defining counter ..................... 51 
5.4 .2 Spectrometer . . . . . . . . . . . . . . . . . . 51

5.4.2.1 Superconducting magnet . . . . . . . . . . . . . . 51

5.4 .2 .2 Prototype tracker . . . . . . . . . . . . . . . 51

5.4.2.3 VLPC cassette with cryocooler . . . . . . . . . . . . . 53

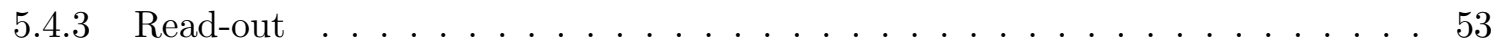

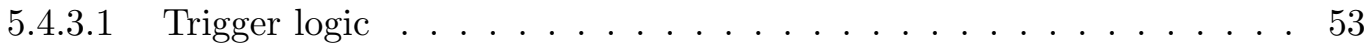

5.4.3.2 Electronics for tracker . . . . . . . . . . . . . . . . 54

5.4 .3 .3 Electronics for beam monitors . . . . . . . . . . . . 56

5.4 .3 .4 DAQ and slow control . . . . . . . . . . . . . 56

5.4 .4 Track reconstruction . . . . . . . . . . . . . . . . 56

5.4 .4 .1 Helical tracking . . . . . . . . . . . . . . . 56

5.4.4.2 Reconstructed momentum distribution . . . . . . . . . . . 57

6 SciFi Tracker Production $\quad 59$

6.1 SciFi stations . . . . . . . . . . . . . . . . . . . . . 59

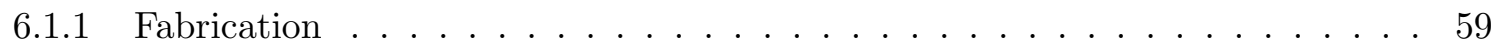

6.1.1.1 Bundling/Connecting . . . . . . . . . . . . . . . 59

6.1.1.2 Gluing/Polishing . . . . . . . . . . . . . . . . . . . . 59

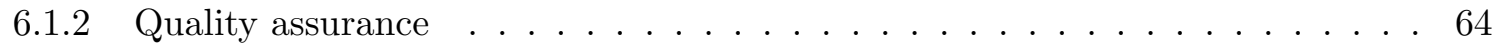

6.1.2.1 Ensuring of bundling/connecting with a LED-CCD system . . . . . 64

6.1.2.2 Light-yield measurement with a ${ }^{57}$ Co radio-active source . . . . . . 64

6.2 Light-guides . . . . . . . . . . . . . . . . . . . . . 70

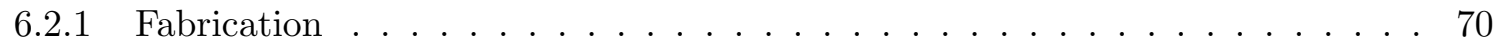

6.2.1.1 Good-fiber selection . . . . . . . . . . . . 76

6.2 .1 .2 Assembling . . . . . . . . . . . . . . . 76

6.2 .1 .3 Gluing . . . . . . . . . . . . . . . . . 77

6.2.1.4 Polishing . . . . . . . . . . . . . . . . 77

6.2 .2 Quality assurance . . . . . . . . . . . . . . . 77

6.2.2.1 Transmittance measurement with a LED-CCD system . . . . . . . . 77

6.2.2.2 Ensuring of channel assignment . . . . . . . . . . . . . . 79

6.3 Tracker assembling and installation . . . . . . . . . . . . . . . . . 79

7 Study of Performance of SciFi Trackers $\quad 83$

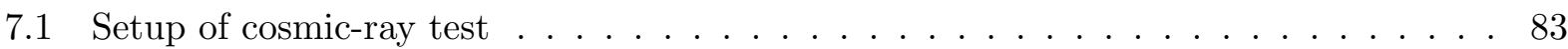

7.2 Tracker read-out . . . . . . . . . . . . . . . . . . . 83

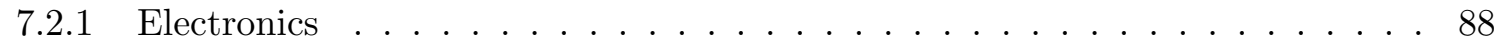

7.2 .2 Trigger $\operatorname{logic} \ldots \ldots \ldots \ldots \ldots \ldots \ldots$

7.2.2.1 Timing adjustment . . . . . . . . . . . . . . . 89

7.2 .3 Tracker slow control . . . . . . . . . . . . . . . . . . . 90

7.2 .4 Tracker DAQ . . . . . . . . . . . . . . . . . 94

7.3 Data taking . . . . . . . . . . . . . . . . . . . 96

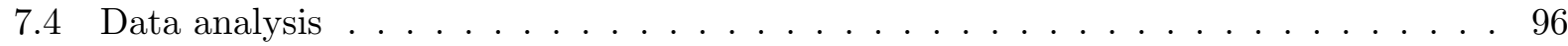

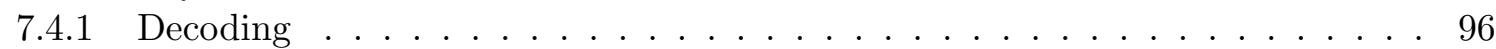

7.4 .2 Calculating light-yield . . . . . . . . . . . . . . . . . 99

7.4.3 Converting VLPC channel number to fiber number . . . . . . . . . . . . . 99

7.4 .4 Finding hits . . . . . . . . . . . . . . . . . . . 99 
7.4 .5 Finding triplets . . . . . . . . . . . . . . . . . . . . . 99

7.4.6 Correcting misalignments . . . . . . . . . . . . . . . . . . 102

7.4.6.1 Correcting misalignments of views . . . . . . . . . . . . . 104

7.4.6.2 Correcting misalignments of stations . . . . . . . . . . . . . . . 104

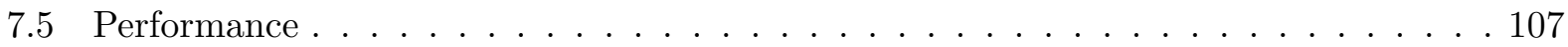

7.5 .1 Alignment . . . . . . . . . . . . . . . . 107

7.5.1.1 Residuals expected by Monte-Carlo simulation . . . . . . . . . . 107

7.5.1.2 Residuals measured by cosmic-ray data . . . . . . . . . . . . . . . . 110

7.5 .2 Efficiency . . . . . . . . . . . . . . . . . 110

7.6 Discussion . . . . . . . . . . . . . . . . . . . . . . 114

8 Discussion $\quad \mathbf{1 2 1}$

8.1 Effects on misalignments . . . . . . . . . . . . . . . . . . . 121

8.2 Status and schedule of MICE SciFi Tracker . . . . . . . . . . . . . . . . 122

9 Conclusion $\quad 125$

$\begin{array}{ll}\text { Acknowledgement } & 127\end{array}$ 


\section{Chapter 1}

\section{Physics Motivations for Neutrino Factory}

\subsection{Neutrino Oscillation Physics}

\subsubsection{Neutrino Oscillation}

It is now well established, from the observation of neutrino oscillations, that neutrinos have mass and mix. Taken together with the present limits on the absolute neutrino mass scale the results indicate that neutrinos are much lighter than all the other fundamental fermions [94], while the values of the mixing angles are also very different from those of the quarks. These facts are difficult to understand in the context of the Standard Model, and it is quite possible that the mechanism that generates neutrino masses and mixing angles may be different to that which generates those of the other fermions [81]. The answer to this puzzle could possibly be found in grand unified theories, which provide a unified description of quarks and leptons at a very high energy scale and could make predictions for the parameters that describe neutrino oscillations. Precise measurements of the oscillation parameters can be used, therefore, to test the ideas of unification, and will perhaps lead to a deeper understanding of the nature of quark and lepton flavour.

The observation of neutrino oscillations also has far-reaching implications in astrophysics and cosmology. The small, but non-zero, neutrino mass may mean that neutrinos contribute as much mass to the universe as all the visible stars [122]. Moreover, the recent KamLAND result strongly indicates that leptonic $\mathrm{CP}$ violation will be observable in neutrino oscillations, possibly leading to an understanding of the observed matter-antimatter asymmetry in the universe. The best, and possibly the only, machine that will allow this discovery is a Neutrino Factory.

None of these fundamental questions can find answers in the physics programme of either LHC or an $e^{+} e^{-}$collider, to which a Neutrino Factory would therefore be completely complementary.

Present results on neutrino oscillations can be readily understood if neutrinos have mass and there is mixing among the three known neutrino flavours; the three neutrino mass-eigenstates $\left(\nu_{1}, \nu_{2}, \nu_{3}\right)$ are different from the three light-neutrino flavour-eigenstates $\left(\nu_{e}, \nu_{\mu}, \nu_{\tau}\right)$ [81], and related to them by the neutrino mixing matrix:

This chapter is cited from Section 1.2 of the Proposal to the Rutherford Appleton Laboratory[1] 


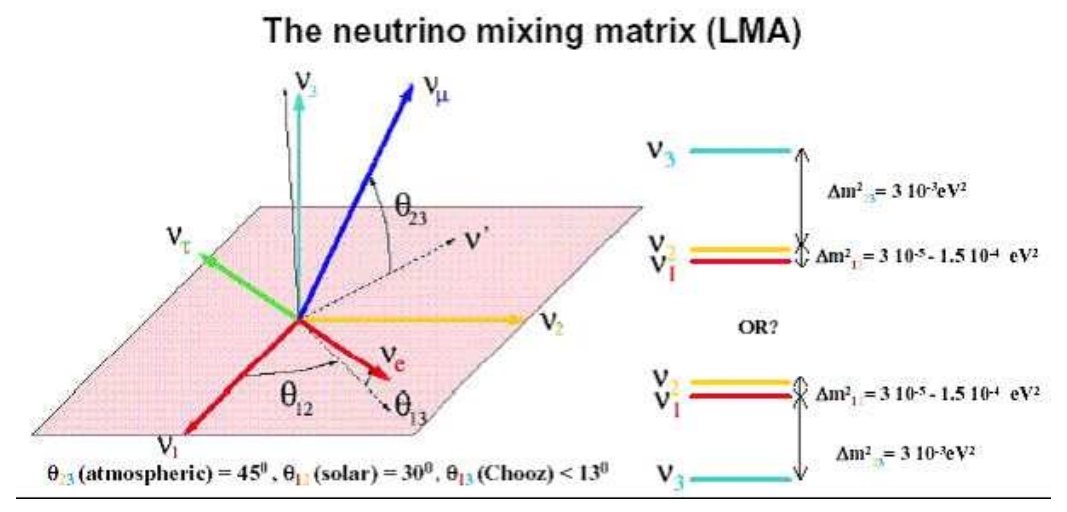

Figure 1.1: a) Rotation of the neutrino mass-eigenstates $\nu_{1}, \nu_{2}$ and $\nu_{3}$ into the flavour eigenstates $\nu_{e}, \nu_{\mu}$ and $\nu_{\tau}$ implied by equation 1.1. The definitions of the Euler angles $\theta_{12}, \theta_{13}$ and $\theta_{23}$ are indicated. b) Neutrino mass spectra allowed by the present data. $\Delta m_{12}^{2}$ is the mass-squared difference that relates to the solar neutrino data, $\Delta m_{23}^{2}$ the one that relates to the atmospheric neutrino data.

$$
U=\left(\begin{array}{ccc}
c_{12} & s_{12} & 0 \\
-s_{12} & c_{12} & 0 \\
0 & 0 & 1
\end{array}\right)\left(\begin{array}{ccc}
c_{13} & 0 & e^{-i \delta} s_{13} \\
0 & 1 & 0 \\
-e^{-i \delta} s_{13} & 0 & c_{13}
\end{array}\right)\left(\begin{array}{ccc}
1 & 0 & 0 \\
0 & c_{23} & s_{23} \\
0 & -s_{23} & c_{23}
\end{array}\right)
$$

The correspondence between the mass eigenstates and the weak eigenstates and the mixing angles that arise in equation (1.1) are depicted in Figure 1.1.a. For three-neutrino flavour oscillations, there are six parameters: the three mixing angles shown in Figure 1.1a, a CP-violating phase parameter, $\delta$, and two mass-squared differences, $\Delta m_{12}^{2}$ and $\Delta m_{23}^{2}$, where $\Delta m_{i j}^{2}=m_{i}^{2}-m_{j}^{2}$. Figure 1.1b shows schematically the two alternatives for the neutrino mass spectrum that are allowed by the present data. Atmospheric neutrino measurements indicate that $\left|\Delta m_{23}^{2}\right|=2 \times 10^{-3} \mathrm{eV}^{2}$ while solar and reactor neutrino measurements indicate that $\Delta m_{12}^{2}=5 \times 10^{-5} \mathrm{eV}^{2}$. The atmospheric neutrino experiments indicate that $\theta_{23}$ is nearly $45^{\circ}$ (maximal mixing), while solar neutrino experiments require $\theta_{12}$ in the range of $25-40^{\circ}$. Reactor experiments indicate that $\theta_{13}$ is smaller than about $10^{\circ}$.

\subsubsection{Forthcoming measurements of the oscillation parameters}

The next few years should produce major improvements in the knowledge of the neutrino oscillation parameters. Further results from SNO and KamLAND are expected soon. The combination of these measurements should, in particular, refine the knowledge of the small mass difference $\Delta m_{12}^{2}$. In addition, several new experiments, SIREN, LENS and HELLAZ, have been proposed to measure the $p p$ solar neutrino spectrum $[113,71,46]$. These experiments should further improve the accuracy with which the solar parameters can be determined.

Over the next ten years, long baseline experiments such as K2K (which has already reported results from its first two years of running), MINOS and the CERN to Gran Sasso (CNGS) experiments will report results $[67,12,51]$. These experiments are expected to confirm the neutrino oscillation interpretation of the Super-Kamiokande atmospheric neutrino results and determine the parameters $\theta_{23}$ and $\Delta m_{23}^{2}$ with an accuracy of about $10 \%$. MINOS and ICARUS will also be sen- 
sitive to $\sin ^{2}\left(2 \theta_{23}\right)$ values as low as 0.04 . In addition to these long baseline experiments there is a proposal to build an off-axis neutrino beam line at the recently approved Japanese Proton Accelerator Research Complex (J-PARC [60]) to illuminate the Super-Kamiokande detector. Discussions have also begun on building such a high-flux beam line using the NuMI beam line at Fermilab [90]. In addition, it is proposed to use the proton driver for an eventual CERN Neutrino Factory, the superconducting proton linac (SPL), initially to provide a low energy super neutrino beam, pointing at the International Frejus Laboratory in the Alps [24]. This would form the first stage of a CERN-based Neutrino Factory complex. These super neutrino beam experiments are likely to bring an improvement of about a factor of 5-20 on the $\theta_{13}$ sensitivity that will be achieved by the MINOS experiment. Such superbeam projects are natural stepping-stones to a Neutrino Factory.

Despite all the improvements in the knowledge of the neutrino parameters that these future experiments will bring, much will remain unresolved. In particular:

1. It will be necessary to determine whether three-flavour mixing is the correct framework or whether sterile neutrinos, neutrino decay or CPT violation also contribute;

2. $\sin ^{2}\left(2 \theta_{13}\right)$ will still be poorly determined (or perhaps unmeasured);

3. the $\mathrm{CP}$ violating phase delta will be unmeasured;

4. the sign of the 23 mass splitting $-\operatorname{sign}\left(\Delta m_{23}^{2}\right)$-is unlikely to be known.

\subsection{Neutrino Factory Concept}

\subsubsection{Neutrino oscillation measurements at a Neutrino Factory}

Physics with the Neutrino Factory has been discussed in several articles [89] and at the Neutrino Factory (NuFact) workshops that have taken place annually since 1999. It is the subject of active investigations by study groups in Europe, Japan, and the US, and during the forthcoming workshops of the NuFact series.

The primary objective of physics at the Neutrino Factory will be the precise measurement of the elements of the neutrino mixing matrix. A neutrino beam derived from the decay of an intense stored-muon beam is the optimum tool for this purpose because it offers substantial advantages over conventional neutrino beam:

1. The energy spectrum of the neutrino beam may be calculated precisely given the muon-beam energy, divergence and polarization.

2. The flavour composition of the neutrino beam is precisely known. Furthermore, the lepton numbers of the neutrino flavours that make up the beam are opposite, so that there is no 'pollution' of the oscillation signals.

3. The neutrino beam is unique in that it contains high-energy electron neutrinos. Not only will this allow the $\nu_{e} \rightarrow \nu_{\mu}$ oscilalitons to be tagged using an experimentally clean 'wrongsign' muon tag, but, since the beam energy will be above the tau-production threshold, the important oscillation channel $\nu_{e} \rightarrow \nu_{\tau}$ can be studied. The ability to study $\nu_{e} \rightarrow \nu_{\tau}$ oscillation is unique to the Neutrino Factory.

4. Changing the polarity of the stored muon beam yields a charge-conjugate neutrino beam. 
5. In the immediate vicinity of the muon storage ring, the neutrino beams are very small and extremely intense.

The Neutrino Factory data set will be diverse, yielding six distinct sub-samples containing events tagged by the appearance of: (i) a right-sign muon; (ii) a wrong-sign muon; (iii) an $e^{+}$ or an $e^{-}$; (iv) a $\tau^{+} ;(\mathrm{v})$ a $\tau^{-}$; or (vi) the absence of a lepton. Measurements can be made with $\mu^{+}$and then with $\mu^{-}$stored in the ring. The most important event samples are those tagged by wrong-sign muons, which provide evidence for oscillations between electron neutrinos and muon neutrinos. For this signal the background rates at a Neutrino Factory are very low, about two orders of magnitude lower than the corresponding rates using conventional neutrino beams. This results in an improvement in experimental sensitivity of about two orders of magnitude. In addition, since the Neutrino Factory will provide intense high-energy beams, oscillation baselines can be very long (thousands of $\mathrm{km}$ ). The muon storage ring can serve two experiments located at significantly different long baselines.

The major physics measurements that will be made with this wealth of data are:

1. Precise determination of $\Delta m_{23}^{2}$ and of the mixing angle $\theta_{23}$;

2. Measurement of the small mixing angle $\theta_{13}$ with a precision of better than half a degree;

3. Observation of the matter effects incurred by electron neutrinos in their passage through the earth. The resulting asymmetry between the rates for $\nu_{e} \rightarrow \nu_{\tau}$ and $\bar{\nu}_{e} \rightarrow \bar{\nu}_{\mu}$ will allow a decisive determination of the sign of $\Delta m_{23}^{2}$;

4. The search for leptonic CP violation through the precise measurement of the $\nu_{e} \rightarrow \nu_{\tau}$ and $\bar{\nu}_{e} \rightarrow \bar{\nu}_{\mu}$ rate asymmetry as a function of energy and baseline.

The superiority of the Neutrino Factory compared with conventional sources of neutrino beams will be demonstrated by considering the determination of $\sin ^{2}\left(2 \theta_{13}\right)$ and the CP violating phase, $\delta$.

The calculated $\sin ^{2}\left(2 \theta_{13}\right)$ sensitivities $(90 \% \mathrm{CL}$ ) of various long baseline neutrino facilities have been estimated in e.g. [72] and are shown in Figure1.2. The sensitivity of a Neutrino Factory is compared with that of the J-PARC-SuperKamiokande project, a higher-energy off-axis project (NUMI off axis), a future high-intensity J-PARC neutrino beam illuminating a megaton water Cherenkov detector (HyperKamiokande) and an 'entry level' Neutrino Factory (one without cooling) of modest intensity. The leftmost ends of the bars indicate the purely statistical sensitivity and demonstrate the analysis power of a Neutrino Factory. Note that the sizes of the systematic errors (indicated by the dark-shaded regions) are well matched to the statistical sensitivities. The Neutrino Factory performance is about two orders of magnitude better than that of either the JPARC-Super-Kamiokande or the NuMi project and about one order of magnitude better than the sensitivity expected if the J-PARC neutrino beam is used to illuminate Hyper-Kamiokande. The range of values of $\sin ^{2}\left(2 \theta_{13}\right)$ allowed by the date increased when correlations with other oscillation parameters are taken into account or the analysis is performed allowing multiple solutions to remain [72]. This limits the Neutrino Factory $\sin ^{2}\left(2 \theta_{13}\right)$ sensitivity to $O\left(10^{-3}\right)$. However, the effect of correlations and degeneracies can be addressed by combining the results of different, complementary, experiments [28], or by using the complementary $\nu_{e} \rightarrow \nu_{\tau}$ transitions [36]. The full sensitivity of a few times $10^{-5}$ can be recovered in such analyses. Hence, Neutrino Factories can achieve sensitivities that are about two orders of magnitude better than can be achieved with conventional high-intensive neutrino beams ('superbeams'). 


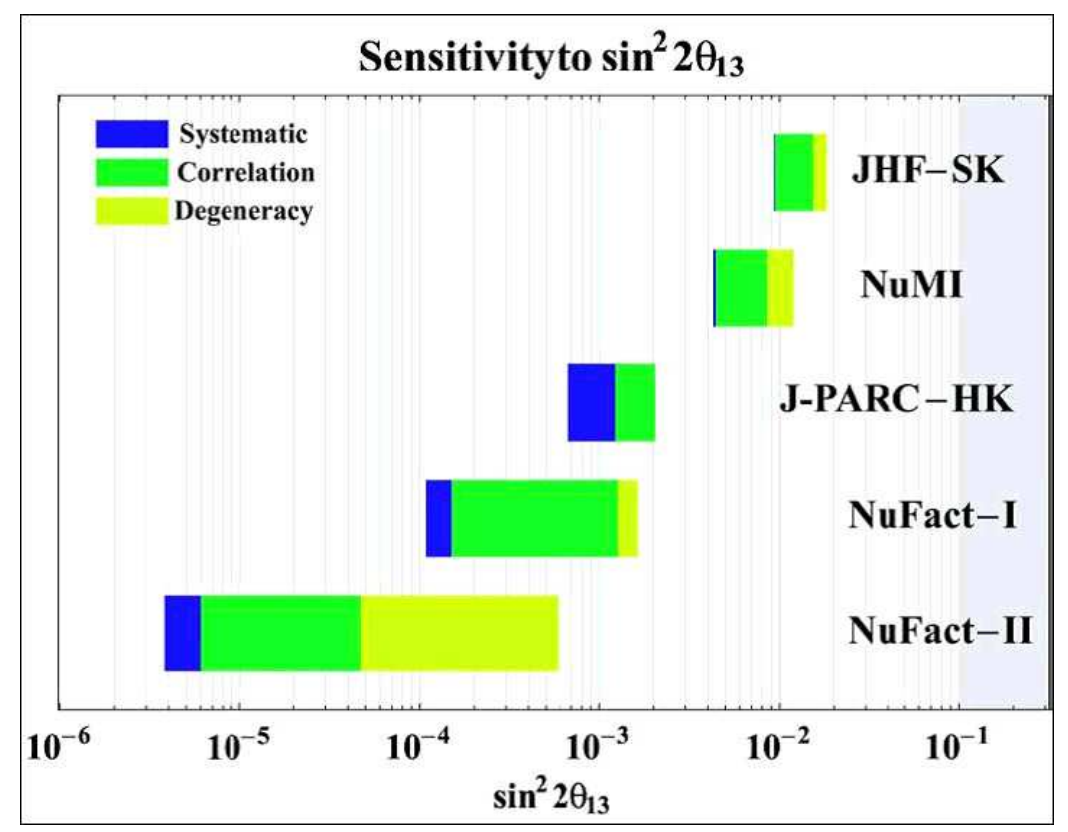

Figure 1.2: Sensitivity of 'entry-level' and high-performance Neutrino Factory (NuFact I and II respectively) $\sin ^{2}\left(2 \theta_{13}\right)$ compared with that of other proposed facilities. For the Neutrino Factory scenarios a single baseline of $3000 \mathrm{~km}, 10^{19}$ (NuFact I) or $2.6 \times 10^{20}$ (NuFact II) muon decays per year at a muon beam energy of $50 \mathrm{GeV}$ are assumed. The correlations and multiple solutions can be addressed at a Neutrino Factory by combining data with those from previous results or by combining complementary measurements. 
If present, $\mathrm{CP}$ violation (CPV) and matter effects will modify the measured $\nu_{e} \rightarrow \nu_{\mu}$ oscillation probabilities. These modifications are different for neutrinos and antineutrinos. The predicted ratio of event rates $N\left(\bar{\nu}_{e} \rightarrow \bar{\nu}_{\tau}\right) / N\left(\nu_{e} \rightarrow \nu_{\tau}\right)$ at a Neutrino Factory experiment with equal $\mu^{+}$and $\mu^{-}$ running is shown as a function of baseline in Figure1.3. With no CPV and no matter effect $(L=0)$ the ratio is 0.5 , reflecting the different neutrino and antineutrino cross sections. As $L$ increases the ratio is enhanced (suppressed) by matter effects if the sign of $\Delta m_{32}^{2}$ is negative (positive). At sufficiently long baselines the matter effects are much larger than effects due to possible CPV (indicated by the bands in the figure). The sign of $\Delta m_{32}^{2}$ and the CP phase delta can therefore be determined by precise measurements of $N\left(\bar{\nu} \rightarrow \bar{\nu}_{\tau}\right) / N\left(\nu_{e} \rightarrow \nu_{\tau}\right)$. However, in this analysis the values of $\left|\Delta m_{32}^{2}\right|, \Delta m_{21}^{2}$, and $\sin \left(2 \theta_{13}\right)$ have been fixed. A fit to determine all these parameters including the effects of correlations and ambiguities show that the Neutrino Factory sensitivity exceeds that of the superbeam projects by $\sim 1-2$ orders of magnitude.

If the LSND oscillation result is confirmed, the simple three-flavour mixing framework will need to be modified to include, for example, additional light neutrinos that are sterile and/or CPT violation. Some information on $\nu_{\mu} \leftrightarrow \nu_{e}$ and $\bar{\nu}_{\mu} \leftrightarrow \bar{\nu}_{\tau}$ oscillations will already be available. It seems likely that there will be a premium on searching for and measuring $\nu_{e} \rightarrow \nu_{\tau}$ oscillations, a programme unique to the Neutrino Factory. It has been shown [20] that there are viable regions of four-neutrino mixing parameter space in which both CPV and thousands of $\nu_{e} \rightarrow \nu_{\tau}$ events could be seen at a Neutrino Factory delivering only $O\left(10^{18}\right)$ decays/yr.

\subsubsection{High-intensity neutrino deep inelastic scattering}

There is a long and rich history of neutrino deep inelastic scattering (DIS) experiments, and structure function measurements from the current generation of experiments are routinely used in 'global fits' for parton distribution functions, sum-rule tests, $\alpha_{S}$ determinations etc. However, precision neutrino DIS physics is more difficult: with neutrino beams from pion decay, large, dense nuclear targets are required (giving unknown heavy-target effects), the energy spectrum of the neutrino beams is relatively poorly determined, and there is an imbalance in the flux of neutrinos and antineutrinos.

At a Neutrino Factory, neutrino DIS would become a high-precision science [6, 74]. $O\left(10^{20}\right) \mu$ decays/year would deliver high-intensity, collimated neutrino and anti-neutrino beams. Even with the compact liquid hydrogen and deuterium targets, the expected event rates would be typically an order of magnitude more than is available from current experiments. The spread in accurately determined (neutrino) beam energies would allow the different structure functions to be disentangled and thus a complete flavour decomposition of the nucleon structure to be performed [19]. The precise determination of the strong coupling constant, $\alpha_{S}$, will be possible from the scaling violations of the structure functions. The 'EMC effect' can be studied using a combination of heavy and light nuclear targets. The ability to tag charm in the final state would allow the CKM matrix elements $\left|V_{c d}\right|$ and $\left|V_{c s}\right|$ to be measured with a precision comparable to the present precision on $\left|V_{u s}\right|$ using processes such as $(d, s)+W^{+} \rightarrow c$. A measurement of $D^{0} \bar{D}^{0}$ mixing could also be made. Finally, with polarized targets, a new realm of high-precision spin physics would open up. The high event rate would allow the determination of the individual flavour components with accuracies of a few percent.

In addition to precision QCD measurements, the electroweak sector of the Standard Model can also be tested at a Neutrino Factory. Particularly important is the extraction of $\sin ^{2}\left(\theta_{w}\right)$ from $\nu_{e}$ and $\nu_{\tau}$ cross sections. For the former, the most accurate current measurements come from the 


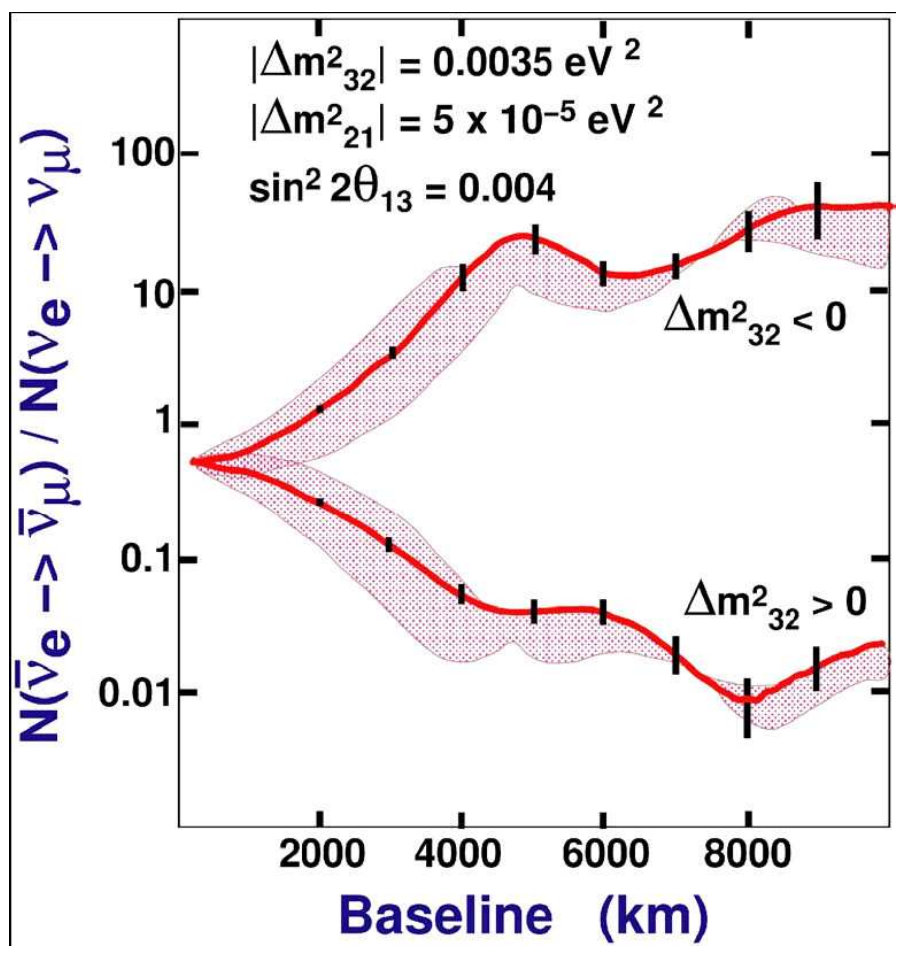

Figure 1.3: Predicted ratios of wrong-sign muon event rates when $\mu^{+}$and $\mu^{-}$are stored in a 20 GeV Neutrino Factory, shown versus baseline. The two bands correspond to the two signs of $\Delta m_{23}^{2}$. The width of the bands show the variation as the CP phase $\delta$ changes from $-\frac{\pi}{2}$ to $+\frac{\pi}{2}$. The thick lines are for $\delta=0$. The statistical errors correspond to a Neutrino Factory providing $10^{21}$ muon decays with a $50 \mathrm{kt}$ detector. 
NuTeV experiment. Studies suggest that the current $(\mathrm{NuTeV})$ error of $\Delta \sin ^{2}\left(\theta_{w}\right) \simeq 0.002$ could be improved by about a factor of 20 at a future Neutrino Factory, with corresponding implications for indirect determinations of the Higgs mass, etc.

Finally, it is important to assess the existing state of knowledge of proton structure at the time when the Neutrino Factory measurements will be made. The HERA $e^{ \pm} p$ collide can access a similar range of information from charged current and neutral current cross sections, over a similar range of the Bjorken scaling-variable, $x$, but typically at much higher four-momentum-transfer squared, $Q^{2}$. The advantages of the Neutrino Factory are: (i) much higher statistics; (ii) target type flexibility; (iii) target polarization. The two machines are clearly complementary in terms of the various types of structure functions that can be measured and the $x, Q^{2}$ range that can be covered.

\subsubsection{Non-neutrino science at a Neutrino Factory}

The Neutrino Factory can be used to produce intense beams of muons with momenta of a few hundred $\mathrm{MeV} / c$ or less and a variety of time structures [16]. These can be used for 'slow-muon' physics studies and will have a flux that will exceed any existing or proposed by slow muon sources by 3-4 orders of magnitude (for the breadth of physics opportunities provided by slow muon sources see, for example, the reviews $[25,32,35])$. This large flux of stopped muons will allow exquisitely sensitive searches for rare muon decays to be carried out, providing stringent tests of the consistency of the Standard Model. In addition, precise determinations of the muon magnetic moment, high statistics studies of muonium and precise measurement of the muon lifetime will allow many parameters of the Standard Model to be determined with unprecedented precision, thus allowing sensitive searches for physics beyond the Standard Model.

Slow muon beams are also of great importance in the study of the atomic and molecular properties of matter. The current intensity of the muon sources available for condensed matter physics research is rather low (typically $\sim 10^{4}$ to $\sim 10^{5}$ muons per second) at ISIS (RAL) and PSI (Switzerland) for experiments measuring time-dependence, although $\sim 10^{6} s^{-1}$ is available at PSI in the most intense beam. The provision of a high intensity muon beam via the Neutrino Factory will allow:

- the study of problems in surface magnetism, polymer and liquid crystal films;

- the study of small samples and time dependent phenomena;

- the elucidation of dynamical effects through the use of high magnetic field level-crossing resonance.

\subsubsection{Neutrino Factory accelerator complex}

As the neutrino beams at a Neutrino Factory will produced from the decay of muons circulating in a storage ring, the primary aim of the accelerator complex is to store as high a muon intensity as possible. This will be achieved using a high power proton source to create intense bunches of protons which are fired into a target. As many of the pions created in this collisions as possible will be captured and transported along a decay channel, where they will decay to muons. The resulting muon beam will have a large size and a large spread in longitudinal and transverse momentum, i.e., a large emittance, which must be reduced to avoid a large fraction of the muons being lost during acceleration and subsequent injection into the storage ring. The reduction of the momentum spread and transverse emittance takes place in two stages, called respectively phase rotation and cooling. 
The muons are then accelerated to a final working energy in a series of accelerators, before being injected into the storage ring.

A number of different designs exist for the Neutrino Factory. Although there are substantial differences between them, each design consists of the same basic components Figure 1.4 shows how these components are laid out in the CERN design. A proton driver produces the very high proton-beam-power (4MW) necessary to achieve the neutrino intensity required for the neutrino oscillation studies. To minimize the longitudinal emittance of the initial muon beam, the proton bunches must be no more than a few nanoseconds long.

Due to the high beam-power and small size, the power density in the target far exceeds that of any comparable facility. Building a target that can withstand the mechanical and thermal stresses that such a beam will create is a major challenge and is the subject of an active R\&D programme. Three different mechanisms have been suggested for focusing and capturing the pions produced in the target: a $20 \mathrm{~T}$ combined superconducting and warm magnet system, a magnetic horn or a wide-aperture bending magnet.

Once captured, the pions decay to produce muons in a decay channel that is $30-40 \mathrm{~m}$ long. To attain the highest muon flux, this channel must have large acceptance for the pions, which implies a combination of wide aperture and strong magnetic field. The most likely way to satisfy these requirements is to use solenoidal magnets, rather than the magnetic quadrupoles that are usually employed for such purposes.

The large momentum spread of the decay muons will be reduced using phase rotation in which early (high energy) particles are de-accelerated and late (low energy) particles are accelerated using, for example, a system of RF cavities. The muons can then be captured into RF bunches, and the transverse emittance reduced using an ionization cooling channel. Ionization cooling is discussed in detail in Chapter 3, but to summarize: the muons are passed through a material, called an absorber, where they lose both longitudinal and transverse momentum. The lost longitudinal momentum is restored using RF cavities following the absorber. The result of this process is a net reduction in transverse momentum spread. However, as well as the cooling coming from the energy loss, there is also heating coming from multiple scattering and the net cooling is a delicate balance between these two effects.

The muons are accelerated to the final working energy, e.g., 20 or $50 \mathrm{GeV}$, using a series of linear accelerators and re-circulating linear accelerators. In the latter, the beam is accelerated through the same RF cavities a number of times, getting the same acceleration in each pass.

Once at the working energy, the muons are injected into the storage ring. It is desirable that the ring satisfy two main requirements. First, as much of the ring as possible should be in the form of straight sections since the decay of the high-energy muons will produce intense neutrino beams primarily in the directions in which these are pointing. Second, the straight sections should point at two or more experiments in different underground laboratories. As shown in Figure 1.4, the favored method of doing this is to make the storage ring a 'triangular' shape.

This thesis is organized as follows. Brief introduction of the ionization cooling is made in Chapter 2. Once explaining the MICE experiment in Chapter 3, the overview of SciFi tracker is described in Chapter 4. The design of the prototype tracker is explained as well as the cosmic-ray and beam tests used with the prototypes in Chapter 5. In Chapter 6, the production of SciFi tracker is described together with quality assurances applied in the construction. The performance of the SciFi trackers tested with cosmic-rays is described in Chapter 7. Finally, the effects of the misalignment found at the trackers to the emittance measurement are discussed in Chapter 8. 


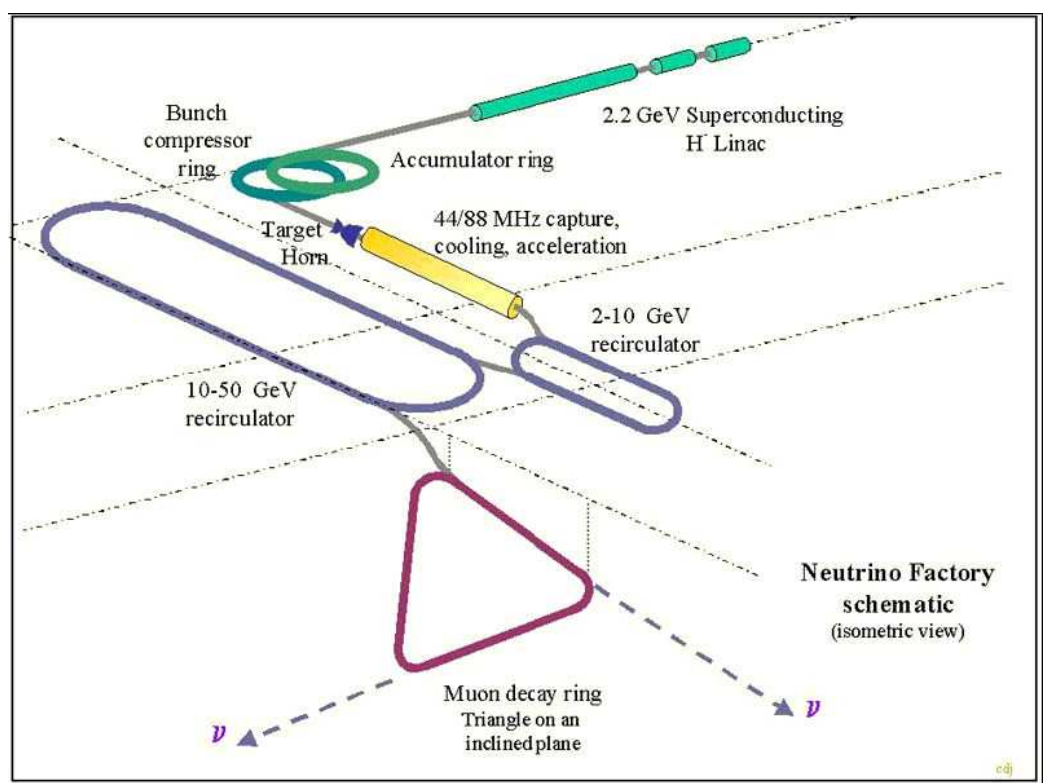

Figure 1.4: Conceptual layout of the various components of a Neutrino Factory complex (CERN scenario). 


\section{Chapter 2}

\section{Principle of Ionization Cooling}

\subsection{Ionization cooling}

The physics performance of a Neutrino Factory is based not only on the clean composition and precisely known flux of the neutrino beam, but also and importantly on the available intensity. The use of ionization cooling increases the density of muons within the volume of phase-space that can be accelerated [57] and improves the performance of the Neutrino Factory by a factor that ranges from 4 to 10 depending on the design $[118,52]$. Although it saves considerably on the cost of the accelerating devices, cooling as is presently envisaged is itself expensive, representing as much as $20 \%$ of the Neutrino Factory cost, estimated from US StudyII [118].

As noted in Section 1, cooling of the transverse phase-space coordinates of a muon beam can be accomplished by passing the beam through energy-absorbing material and accelerating structures, both embedded within a focusing magnetic lattice; this is known as ionization cooling [114, 84]. Other cooling techniques (electron, stochastic, and laser cooling) are far too slow to yield a significant degree of phase-space compression within the muon lifetime. Ionization of the absorbing material by the muons decreases the muon momentum while (to first order) not affecting the beam size; at the same time, multiple Coulomb scattering of the muons in the absorber increases the beam divergence, heating the beam.

Cooling is discussed quantitatively in terms of emittance $\varepsilon$, defined as the volume occupied by a beam in phase space. This can be expressed as $\varepsilon=\sqrt{D}$, where $\mathrm{D}$ is the determinant of the 6 dimensional covariance matrix of the beam particles in the $6 \mathrm{D}$ coordinates $(x, y, t, d x / d z, d y / d z, c d t / d z)$. Normalized emittance $\varepsilon_{n}$ is obtained by using the coordinates $(x, y, t, p / m c . d x / d z, p / m c . d y / d z$, $p / m . d t / d z)$. The same calculation performed in the $4 \mathrm{D}$ space of spatial coordinates yield the transverse $4 \mathrm{D}$ emittance. The transverse emittance is defined as the emittance in one $2 \mathrm{D}$ plane $(x, d x / d z)$; in a solenoid channel, the cylindrical symmetry argues for this to be calculated as the square-root of the transverse $4 \mathrm{D}$ emittance. The longitudinal emittance is defined similarly in the time-energy dimensions.

Within an absorber, normalized transverse emittance $\varepsilon_{n}$ behaves approximately as [85]

$$
\frac{d \varepsilon_{n}}{d s}=-\frac{1}{\beta^{2}}\left\langle\frac{d E_{n}}{d s}\right\rangle \frac{\varepsilon_{n}}{E_{n}}+\frac{1}{\beta^{3}} \frac{\beta_{\perp}(0.014)^{2}}{2 E_{\mu} m_{\mu} X_{0}},
$$

This chapter is cited from Chapter 2 of the Proposal to the Rutherford Appleton Laboratory[1] 
where $s$ is path length, angle brackets denote mean value, muon energy $E_{\mu}$ is in $\mathrm{GeV}, X_{0}$ is the radiation length of the absorber medium in $\mathrm{m}, \beta_{\perp}$ is the optical beta-function in the magnetic channel in $\mathrm{m}$, and $\beta$ is the particle velocity. This expression is appropriate to the cylindricallysymmetric case of solenoid focusing, where $\beta_{x}=\beta_{y}=\beta_{\perp}$. The first term in this equation is the cooling term and the second is the heating term.

To minimize the heating term, which is proportional to $\beta_{\perp}$ and inversely proportional to radiation length, it has been proposed [9] to use liquid hydrogen as the energy-absorbing medium, with $d E / d s=30 \mathrm{MeV} / \mathrm{m}$ and $X_{0}=8.7 \mathrm{~m}$, and to use superconducting solenoid focusing to give a small value of $\beta_{\perp} \sim 10 \mathrm{~cm}$, rather than quadrupoles; this corresponds to large beam divergence at the location of the absorbers, so that scattering in the absorbers gives a relatively small contribution to the emittance. Key issues in absorber $\mathrm{R} \& \mathrm{D}$ include coping with the large heat deposition by $\mathrm{RF}$ dark currents and the intense $\left(10^{14}\right.$ muons per second) muon beam of the Neutrino Factory, while minimizing scattering in absorber-vessel windows, which are by necessity of higher-Z material.

An additional technical requirement is high-gradient re-acceleration of the muons between absorbers to replace the lost energy, so that the ionization-cooling process can be repeated many times. Ideally, the acceleration should exceed the minimum required for momentum replacement, allowing 'off-crest' operation. This gives continual rebunching, so that even a beam with large momentum spread remains captured in the RF bucket. Even though it is the absorbers that actually cool the beam, for typical accelerating gradients (around $10 \mathrm{MeV} / \mathrm{m}$ ), the RF cavities dominate the length of the cooling channel (see e.g. Figure 2.1). The achievable RF gradient determines how much cooling is practical before an appreciable fraction of the muons have decayed or drifted out of the bucket.

It follows from the above equation that the percentage decrease in normalized emittance is proportional to the percentage energy loss. Low beam energy is favored because it requires less re-accelerating voltage. The negative slope of $(d E / d x) / E[94]$ at low energies leads to longitudinal heating, while the positive slope at high energies comes with an increase in straggling. So, most muon-cooling designs and simulations to date have used a momentum near the ionization minimum, between 150 and $400 \mathrm{MeV} / \mathrm{c}$. This is also the momentum range in which the pion-production crosssection of thick targets tends to peak, and is thus optimal for muon production as well as cooling.

Intuition, calculations using linear ionization cooling theory and detailed simulations indicate that the process, if effected in a homogeneous magnetic field, will decrease the transverse momentum of the muons, but not the beam size itself. In order to convert the reduction of angles into a reduction of beam size, a change in optical functions is necessary. This can be done in various ways, the most drastic one being to perform a magnetic field reversal, which can also be used as a way to reduce the beta function. The beta function can be squeezed in a periodic way by repeated field reversals (FOFO or SFOFO cooling cells), or only a few times during the cooling process (single- or double-flip cooling channel [18]). The difficulty in the design of a cooling channel is to integrate the three basic element, low-Z absorbers, RF cavities and solenoid focusing, in the most compact and economical way.

\subsection{Cooling channel design}

There have been many iterations of cooling section designs, both in the US and European studies. A cooling device desirable for experimental test is a section of a cooling channel from a viable high-performance Neutrino Factory design. It is defined by a few important characteristics: 
1. The cooling factor. The transverse emittance reduction in a short cooling section is at best a factor $\Delta \varepsilon / \varepsilon=\Delta E / E$, where $\Delta E$ is the average energy loss in the absorbers (and restored in the RF cavities) and $E$ is the average particle kinetic energy. For muons of $200 \mathrm{MeV} / \mathrm{c}$, a '10\% cooling experiment' requires an energy loss of about $20 \mathrm{MeV}$ and similar gain in the RF system.

2. The RF system, characterized in particular by its frequency. There are several existing scenarios: in the scheme developed for the US Study II [118], cooling is performed with 201 $\mathrm{MHz}$ cavities; in the scheme developed at CERN the cooling is performed at $88 \mathrm{MHz}$. These differences in Neutrino Factory design are motivated by the different preparation of the beam prior to the cooling section. Another crucial parameter to consider for the cooling channel is of course the gradient that can be achieved with such RF systems. Cooling experiment designs have been developed in which the frequency is $88 \mathrm{MHz}$ or $201 \mathrm{MHz}$. While the choice for the cooling experiment proposed here $(201 \mathrm{MHz})$ has been made on the basis of performance and practicality [2], the experience gained will be beneficial for any of the schemes currently contemplated.

3. The beam to be cooled. It is characterized by its average energy, energy spread, beam size and angular divergence. In a Neutrino Factory design, the beam has properties that vary along the cooling channel. One should vary the beam characteristics in a test experiment to reproduce this variety of conditions all the way down to the equilibrium emittance. Here is an example of typical beam properties:

- Momentum $200 \mathrm{MeV} / \mathrm{c}$

- Momentum spread $\pm 10 \%$

- Beam size $5 \mathrm{~cm}$ rms in both projections

- Beam angular divergence $150 \mathrm{mrad} \mathrm{rms}$ in both projections

4. The magnetic field and diameter of the magnetic channel, typically $4 \mathrm{~T}$ field with an aperture of $15 \mathrm{~cm}$ radius.

One of the characteristics of a cooling channel is the equilibrium emittance. A beam at equilibrium emittance would traverse the channel without net reduction or increase of its emittance. A precise measurement of this quantity for various configurations of magnetic field and beam momentum, and comparison with that expected from simulation, given approximately by

$$
\varepsilon_{n}^{(e q u .)}=\frac{\beta_{\perp}(0.014)^{2}}{2 \beta m_{\mu} \frac{d E_{\mu}}{d s} X_{0}}
$$

is one of the quantitative aims of the experiment.

A detailed, integrated simulation of the beam line, instrumentation and cooling apparatus is essential for the success of the Muon Ionization Cooling Experiment. The performance measured with MICE will be compared with the performance calculated from the detailed simulations. Three distinct computer codes are used: ICOOL [39], developed at BNL, a beam line simulation toolbox [22] based on Geant4 [44], developed at FNAL, and PATH [93], developed at CERN. These codes have been used extensively in ionization cooling design studies and their results are in excellent agreement. 

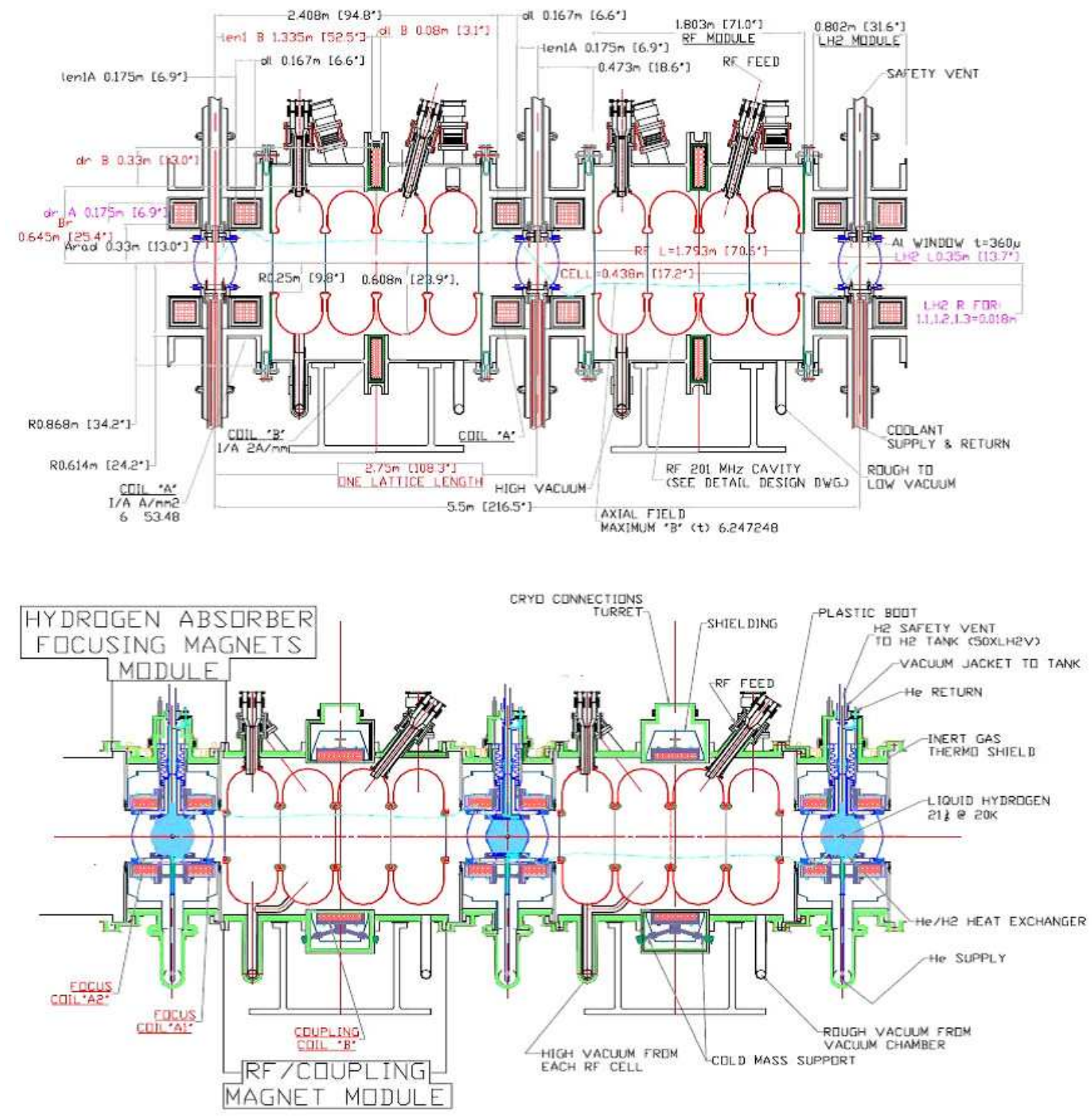

Figure 2.1: Top: Engineering rendering of the $201 \mathrm{MHz}$ cooling channel (from US Neutrino Factory Feasibility Study II [118]). Shown in cross section are three liquid-hydrogen absorbers, each enclosed within a pair of 'focusing' solenoids, interspersed with two 4-cavity $201 \mathrm{MHz}$ RF assemblies, each encircled by a 'coupling' solenoid. Bottom: engineering rendering of the cooling section of MICE. The radius of the coils is reduced to save cost, while the safety windows and argon jacket are motivated by compliance with safety requirements. 


\section{Chapter 3}

\section{The International Muon Ionization Cooling Experiment (MICE)}

\subsection{Layout of the experiment}

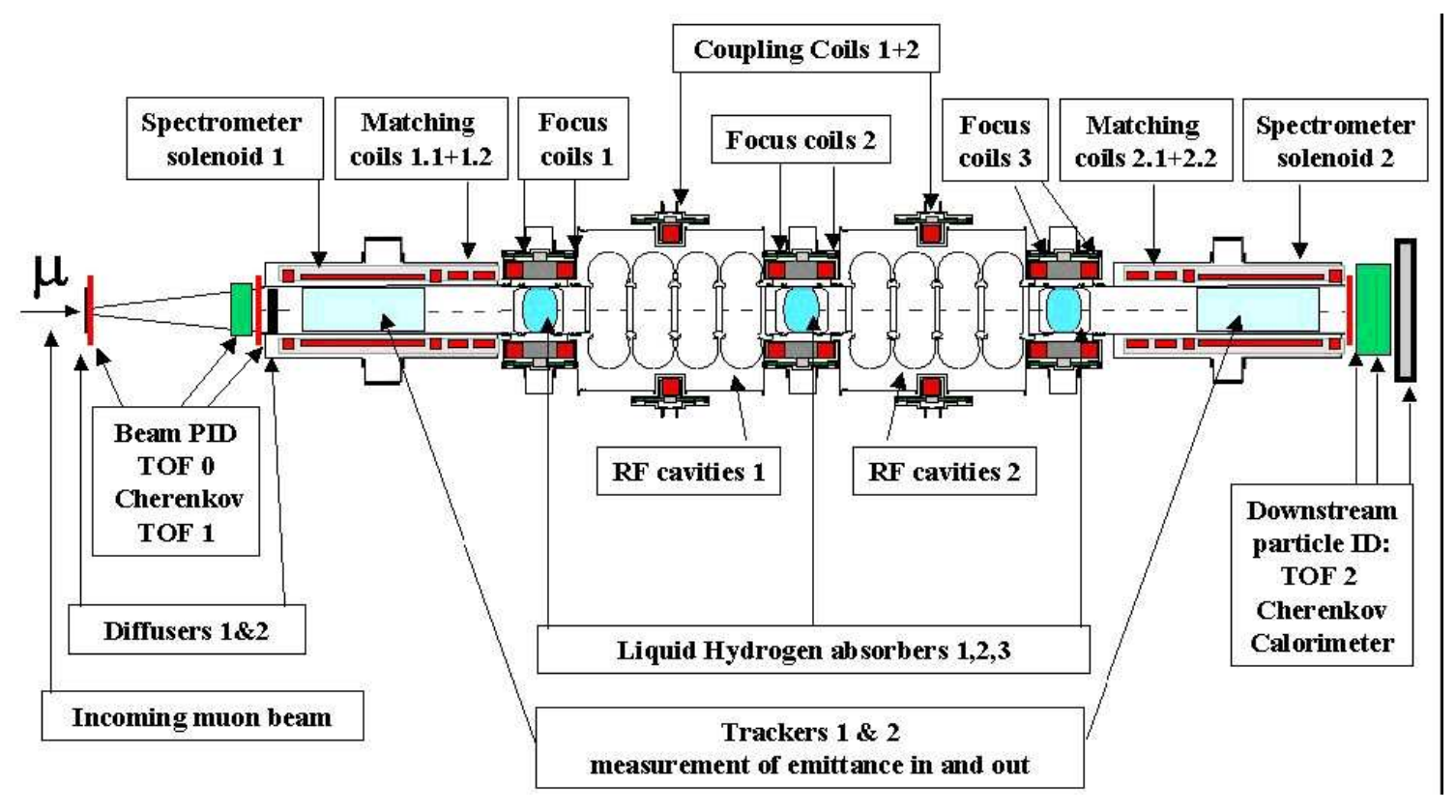

Figure 3.1: Artist's view of the International Muon Ionization Cooling Experiment (MICE), with the beam entering from the left.

The main components of MICE are outlined in Figure 3.1. Cooling is provided by two cells from the first part of the $201 \mathrm{MHz}$ cooling channel. The incoming muon beam encounters first a beam preparation section, where a pair of high-Z (lead) diffusers generates a tunable input emittance. In this section, a precise time measurement is performed and the incident particles are identified.

Section 3.1, 3.2, 3.3 and 3.4 are cited from the Proposal to the Rutherford Appleton Laboratory[1] 
There follows a first spectrometer, in which momentum, position and angles of each incoming particle are measured by means of tracking devices embedded in a uniform-field solenoid.

Next comes the cooling section itself, with hydrogen absorbers and RF cavities, the focusing optics being provided by superconducting coils. The default magnetic configuration is such that the angles of the outgoing particles are measured in a second spectrometer, identical to the first one. At the downstream end of the experiment, another time-of-flight (TOF) measurement is performed, and particle identification by means of Cherenkov counter and a calorimeter eliminates muons that have decayed in the apparatus.

The positions and operating parameters of the elements of MICE are summarized in Table 3.1. All simulations in this section have been performed based on these values.

As noted, the cooling experiment consists of two complete cooing channel cells. One additional absorber finishes the cooling section, both for reason of symmetry and to protect the trackers against dark currents emitted by the RF cavities. To avoid emittance growth, the magnets in these cells must be matched to the spectrometer solenoids. This is done using two sets of matching coils situated between the solenoids and the focus pairs. Correction coils around each spectrometer solenoid ensure a uniform field of $4 \mathrm{~T}$ for a length of $1 \mathrm{~m}$.

Figure 3.2 shows the matched beta function for the central momentum of $200 \mathrm{MeV} / \mathrm{c}$. It is essentially constant in the detector, indicating that the required matching is achieved. As will be seen in Section xx, the experiment can be matched within a momentum range from 140 to 240 $\mathrm{MeV} / \mathrm{c}$, and the beta function at the absorbers can be tuned down to a few centimeters.

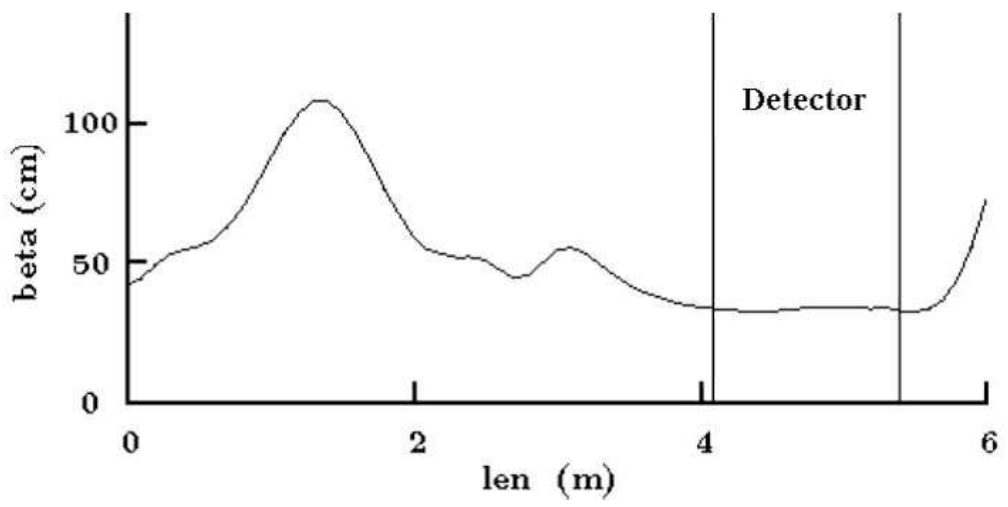

Figure 3.2: Beta function vs length through the match. The coordinate $\mathrm{z}=0$, around which this function is symmetrical, is at the middle of the central hydrogen absorber 2 .

\subsection{Beam dynamics and quantities to be measured}

This section describes the behavior of the beam in the channel and delineates the quantities that could be measured to characterize it. Following earlier simulations of the cooling experiment with path both for the $88 \mathrm{MHz}$ and the $201 \mathrm{MHz}$ designs [7, 40], the simulations presented here were performed with ICOOL $[39,57]$, which includes energy loss, multiple scattering, straggling in the hydrogen absorbers and realistic descriptions of all magnetic fields.

The simulation includes $0.5 \mathrm{~mm}$ thick aluminum absorber windows (their thickness has been increased with respect to that of Study II to mimic the effect of safety windows), and stepped 


\begin{tabular}{|c|c|c|c|}
\hline Item & $z_{0}[\mathrm{~mm}]$ & $\Delta z[\mathrm{~mm}]$ & properties \\
\hline Diffuser 1 & -10000 & 1.4 & lead \\
\hline TOF 0 & -9950 & 50 & plastic scintillator $\sigma(t)=50 \mathrm{ps}$ \\
\hline TOF 1 & -50 & 50 & plastic scintillator $\sigma(t)=50 \mathrm{ps}$ \\
\hline Diffuser 2 & 0 & 22.4 & lead \\
\hline Correction coil 1.1 & 500 & 120 & \\
\hline Spectrometer solenoid 1 & 680 & 1260 & \\
\hline Tracker 1 & 810 & 1000 & uniform-field region, $\mathrm{B}=4 \mathrm{~T}$ \\
\hline Correction coil 1.2 & 2000 & 120 & \\
\hline Matching coil 1.1 & 2269 & 202 & \\
\hline Matching coil 1.2 & 2519 & 202 & \\
\hline Focus coil 1.1 & 3042 & 200 & \\
\hline Hydrogen absorber 1 & 3187 & 350 & energy loss $11 \mathrm{MeV}$ \\
\hline Focus coil 1.2 & 3482 & 200 & \\
\hline RF 1 & 3877 & 1720 & $\begin{array}{l}4 \text { 201-Mhz cavities separated by Be windows; } \\
\text { accelerating gradient } 8.3 \mathrm{MV} / \mathrm{m}\end{array}$ \\
\hline Coupling coil 1 & 4567 & 350 & \\
\hline Focus coil 2.1 & 5792 & 200 & \\
\hline Hydrogen absorber 2 & 5937 & 350 & energy loss $11 \mathrm{MeV}$ \\
\hline Focus coil 2.2 & 6232 & 200 & \\
\hline $\mathrm{RF} 2$ & 6627 & 1720 & $\begin{array}{l}4 \text { 201-Mhz cavities separated by Be windows; } \\
\text { accelerating gradient } 8.3 \mathrm{MV} / \mathrm{m}\end{array}$ \\
\hline Coupling coil 2 & 7317 & 350 & \\
\hline Focus coil 3.1 & 8542 & 200 & \\
\hline Hydrogen absorber 3 & 8687 & 350 & energy loss $11 \mathrm{MeV}$ \\
\hline Focus coil 3.2 & 8982 & 200 & \\
\hline Matching coil 2.2 & 9503 & 202 & \\
\hline Matching coil 2.1 & 9753 & 202 & \\
\hline Correction coil 2.2 & 10104 & 120 & \\
\hline Spectrometer solenoid 2 & 10284 & 1260 & \\
\hline Tracker 2 & 10414 & 1000 & uniform-field region, $\mathrm{B}=4 \mathrm{~T}$ \\
\hline Correction coil 2.1 & 11604 & 120 & \\
\hline Cherenkov & 11824 & 560 & aerogel $\mathrm{n}=1.02$ \\
\hline TOF 2 & 12390 & 50 & plastic scintillator $\sigma(t)=50 \mathrm{ps}$ \\
\hline Calorimeter & 12900 & 150 & scintillating-fibre lead spaghetti calorimeter \\
\hline
\end{tabular}

Table 3.1: Position along the beam line and operating parameters of the elements of MICE 
beryllium windows. The RF fields used are those from perfect pillbox cavities. The limiting apertures are found to be located at the central iris of each 4-cavity RF assembly. The RF gradients are $8.3 \mathrm{MV} / \mathrm{m}$ and the phase is such that maximum acceleration is obtained in each cavity. An illustration of the elements of the experiment is given in Figure /reffig:3.3.

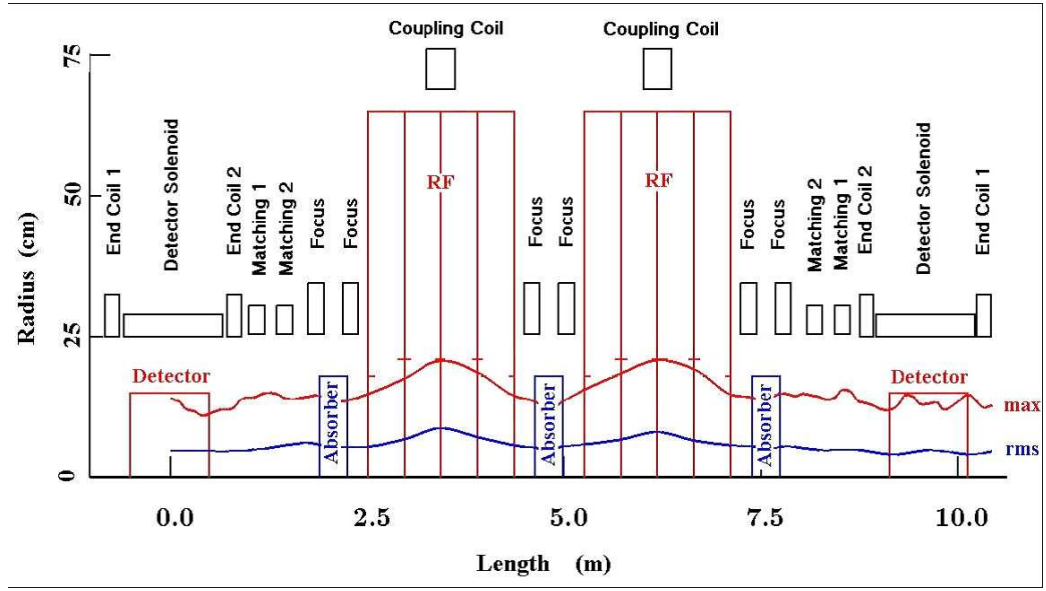

Figure 3.3: Description of the essential optical elements of MICE. Also shown are the rms beam radius and beam envelope for a beam of the same emittance as at the entrance of the cooling channel in Study-II.

Figure 3.4 shows the expected behavior of the beam for a large input emittance. The kinetic energy of the beam is reduced at each passage through an absorber, and increased in the RF cavities. At the location of each of the absorbers, the normalized emittance decreases. (It does not decrease through the accelerating section, but the un-normalized emittance would.) As an extra absorber has been included, the momentum of the outgoing beam is reduced with respect to the incoming. This could be avoided by emptying the central absorber. The experiment must measure the emittances of the incoming and outgoing beams and, most importantly, their ratio, with a precision much better than the expected $10 \%$ reduction in emittance.

Figure 3.5 shows how the output emittance varies with that of the input beam. For very large input emittance, the output emittance is reduced substantially, but the transmission decreases, large-amplitude particles being lost in the channel. Nevertheless, this may not be a sign of poor performance of the device: what really matters is the variation of the number of particles within a desired acceptance. This is shown in Figure 3.6, where the cooling performance is defined by comparing the number of particles in a given acceptance (here the acceptance of a subsequent muon accelerator) before and after the cooling section. In both figures it can be seen that, as the input emittance falls, so does the cooling performance. At very low input emittance, the beam is heated rather than cooled. The equilibrium emittance is the boundary between the two cases, the value for which the input and output emittances are equal. Ideally the equilibrium emittance should be as small as possible, and the acceptance as large as possible.

\subsection{Measurement technique}

The diagnostic devices of MICE are principally aimed at measuring, before and after the cooling channel, the volume occupied by the muon beam in six-dimensional phase space. The measurement 


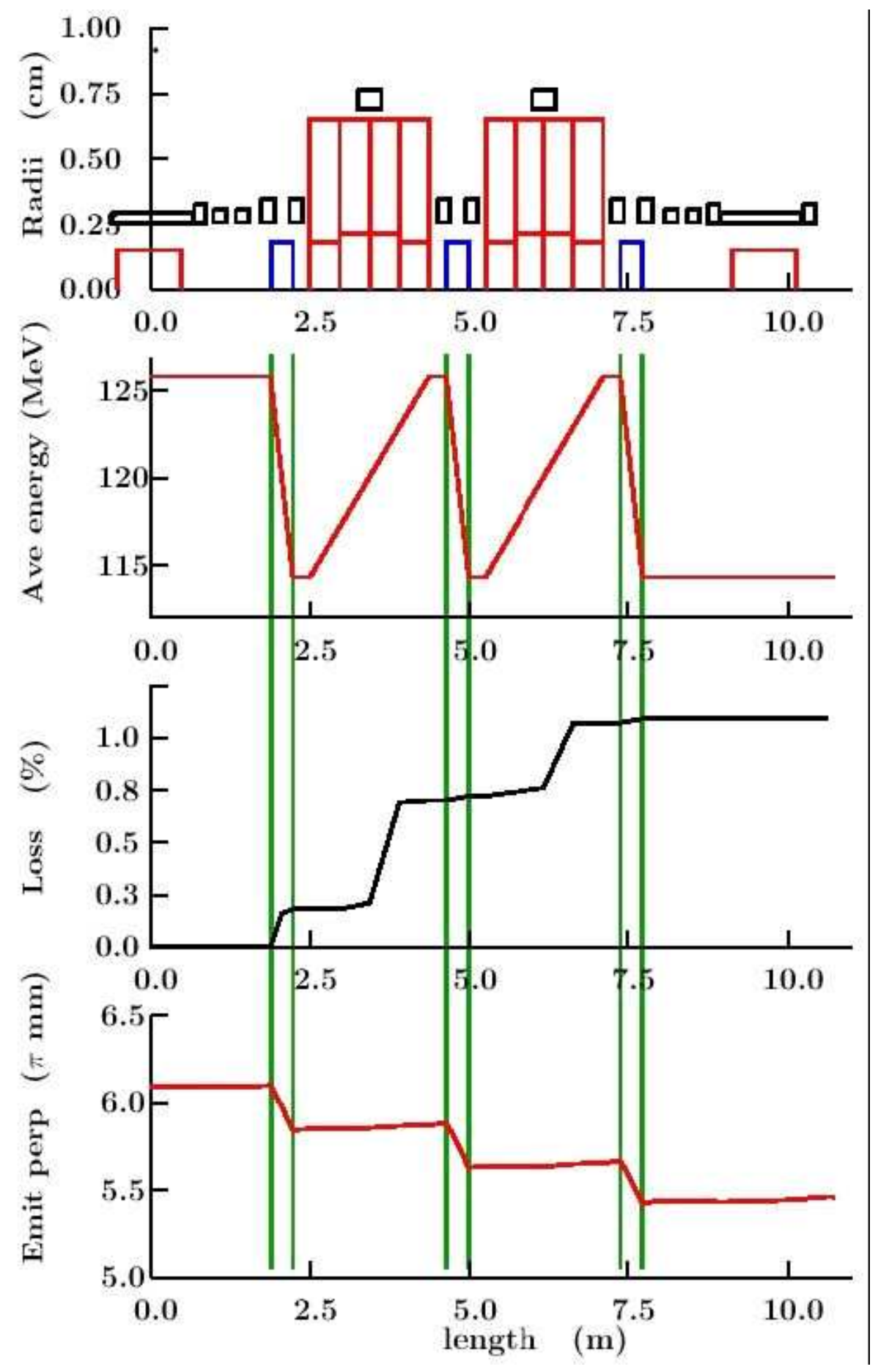

Figure 3.4: Properties of the beam along the experiment. The narrow vertical lines depict the locations of the hydrogen absorbers. From top to bottom: the experiment layout, the average muon kinetic energy showing the typical saw-tooth, the particle losses which occur mostly at the central RF cavity iris and the 2D normalized transverse emittance, which is seen to decrease in the absorbers. These are all plotted for an input emittance of $6.1 \pi \mathrm{mm} \mathrm{rad}$ and muons of $200 \mathrm{MeV} / \mathrm{c}$ average momentum. 

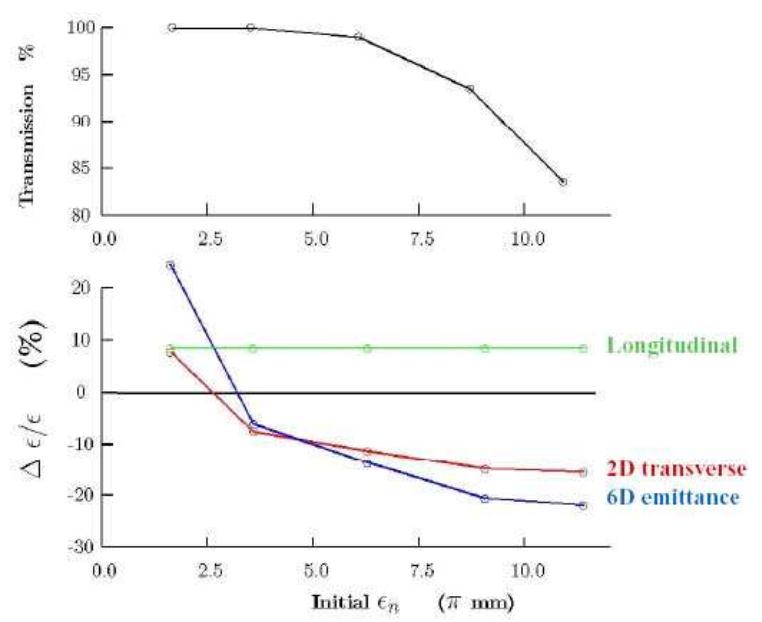

Figure 3.5: Transmission and decrease of emittance vs. input normalized emittance. The equilibrium emittance is around $2.5 \pi \mathrm{mm}$ rad. The transmission plot shows that scraping becomes significant above an input emittance of $6 \pi \mathrm{mm}$ rad.

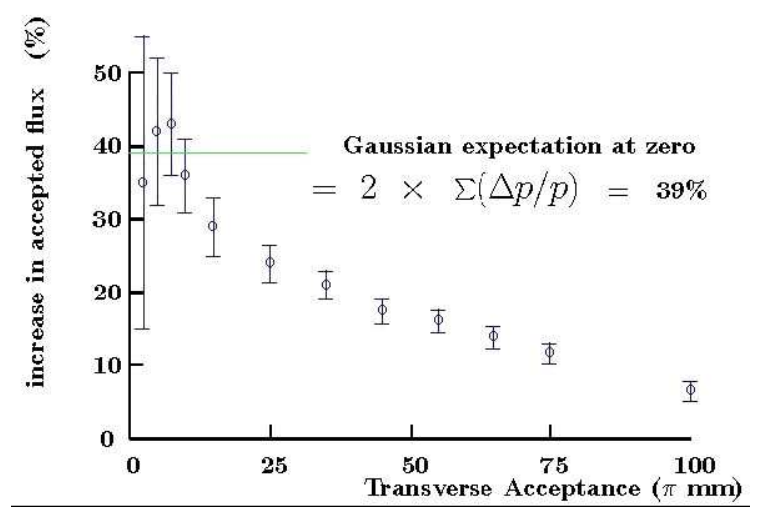

Figure 3.6: Cooling channel efficiency, measured as the increase in the number of muons inside a given transverse normalized acceptance, for an input beam of large emittance. For the acceptance of the Neutrino Factory muon accelerator, which is $15 \pi \mathrm{mm}$ rad (normalized) the increase in the number of accepted muons when going through MICE would be $\geq 30 \%$. The acceptance is 3 times larger than the rms beam size, which is proportional to the square-root of emittance; this implies a scale factor of 9 in the abscissa compared to that of Figure 3.5. 
system must not only measure emittances, but also count particles. Two techniques have been considered: i)the multi-particle method, where emittance and number of particles in any given area of phase space are determined from the global properties of a bunch; ii) the single-particle method, in which the properties of each particle are measured. The full determination of the covariance matrix in six dimensions is a delicate task in a multi-particle experiment, and the desired diagnostics would have to be developed specifically; for these reasons, the single particle-method was preferred. This technique, typical of particle physics experiments, is also one for which experimental methods already exist. Furthermore if follows exactly P.Lapostolle's definition of the rms emittance [68].

In a particle-by-particle experiment, the properties of each particle are measured before and after the cooling channel in a magnetic spectrometer. Each detector measures, at given $z$ positions, the coordinates $x$ and $y$ of every incident particle, and possibly the time. Momentum and angles are reconstructed by using more than one plane of measurement. For the experimental resolution not to affect the measurement of the emittance by a significant factor, the rms resolution of the measurements must be better than about $10 \%$ of the rms beam size at the equilibrium emittance in each of the six dimensions. An essential aim of MICE is to measure the equilibrium emittance precisely.

There will be only one particle traveling through the apparatus within the time window of the system. For each incident particle it will be possible to determine whether it was lost in the channel, or if it went through successfully, by the presence at an appropriate time (taking account of the travel time and fluctuations thereof) of a particle in the downstream spectrometer. In this case the losses can be very cleanly separated, particle-by-particle, from the effect of cooling.

Except for possible collective effects such as space charge, this particle-by-particle technique is equivalent to full-beam measurements, but offers several advantages:

- Correlations between parameters can be easily measured if the 6 parameters are measured on a particle-by-particle basis.

- The detailed understanding of the role of each beam parameter, and in particular of the energy, transverse momentum and RF phase (=time) of the particles, can easily be studied by making selection cuts in the ensemble of particles without making changes to the beam parameter settings.

- Software cuts based on the incoming beam make it furthermore possible to derive a variety of results with different input beam conditions from a single data set.

- Any desired input beam conditions can be reconstructed by appropriate weighting or culling of the observed particles. The weights or cuts can be determined by comparing the distribution in phase space of particles observed in the first spectrometer to the desired distribution.

Energy measurement requires a magnetic spectrometer, which could be designed in a variety of ways. Simplicity in the matching with the cooling section and the need to keep a large-emittance beam in a small physical volume led to the choice of solenoid magnets coaxial to the cooling channel. The particle tracking inside the solenoid, obtained by measuring successive points along the trajectory, allows position, angles and the momentum of each charged particle to be determined as depicted in Figure 3.7.

Three $(x, y)$ measurements in each spectrometer with particle detectors placed perpendicular to the muon beam and at three different $z$ values are sufficient to determine the muon position, angles and momentum at the entrance and exit of the cooling channel. To obtain the best resolution and 


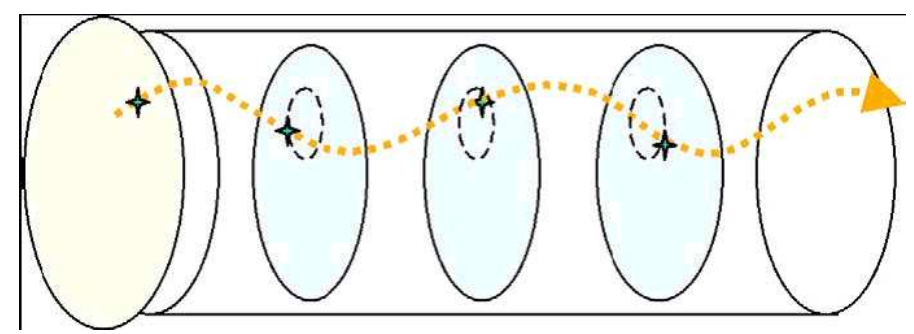

Figure 3.7: The space coordinates at three (or more) planes within the magnetic field and the TOF information provide a complete reconstruction of the $6 \mathrm{D}$ phase space coordinates for each particle.

the largest dynamic range, the spectrometer length should be such that the average muon makes about $2 / 3$ of a turn from the beginning to the end, i.e., about $1 \mathrm{~m}$ for $200 \mathrm{MeV} / \mathrm{c}$ muons in a $4 \mathrm{~T}$ solenoid. To avoid reconstruction ambiguities, more than three planes of detectors will be used. As indicated in Figure 3.1, the time coordinate is determined with precise time-of-flight measurements at the entrance and at the exit of the channel. Measurement of the RF phase of each incoming particle with a precision of $5^{\circ}$ requires a time measurement resolution of $70 \mathrm{ps}$ or better for the 201 $\mathrm{MHz}$ RF system, making precision time-of-flight counters compulsory.

The design of MICE, with spectrometer solenoids matched directly to the cooling cells, allows a small spectrometer to measure a large phase space and introduces no artificial energy dispersion. It has, however, one major drawback. The single-particle detectors will be exposed almost directly to a large dark current and x-ray background generated by the nearby high-gradient RF cavities. The understanding of this problem is well underway and described in Section 3.5. Several factors contribute to protect the tracking detectors: i) the RF cavities will be operated at a moderate gradient of $8.3 \mathrm{MV} / \mathrm{m}$, due to the limited availability of RF power; ii) the dark-current electrons must pass through the liquid-hydrogen absorbers, which are thick enough to absorb them completely, letting through x-rays only; iii) the detectors are built of low-Z material and are well able to distinguish hits from muons from those generated by x-rays. Thus, it appears that, at the RF voltage at which the MICE cavities will be operated, the performance of the detectors will not be affected. Studies of this critical issue are presented in Section 3.5; these will continue with high priority.

In addition to the primary goal of measuring the space-time coordinates and the momenta of incoming and outgoing muons, the diagnostic devices should also be capable of identifying and rejecting (i) residual undecayed pions and (ii) decay electrons, in order to restrict the emittance measurement to that of the muon beam exclusively. Due to the different kinematics, even a small fraction of these backgrounds, $>0.1 \%$, has a detrimental effect on the emittance resolution. Pion identification can be achieved by means of a precise time-of-flight measurement over a $10 \mathrm{~m}$ distance prior to the first spectrometer solenoid. A 50 ps time resolution, associated with an $(x, y)$ measurement, leads to a $99 \%$ pion rejection. The decay electrons can be identified from their specific kinematic properties with $80 \%$ efficiency. A larger rejection is needed and requires specific electron identification with, e.g., a Cherenkov detector or a range measurement. 


\subsection{Tracker}

\subsubsection{Requirements}

Three precise measurement planes can provide adequate resolution in transverse and longitudinal momentum. Nonetheless, the tracking issues for single muons in MICE can become very problematic if the x-ray background from the RF cavities is too severe. The tracking detectors are therefore required to have low mass, to avoid photon conversions, and enough redundancy to separate signal hits from a potentially large background of photon hits.

The scintillating fiber tracker is the baseline option for the MICE Fiber Tracker. It has a number of powerful features:

1. it can operate effectively at very high background rates

2. it is immune to electromagnetic interference

3. it is totally passive and therefore poses little risk when operated near liquid hydrogen

\subsubsection{Layout}

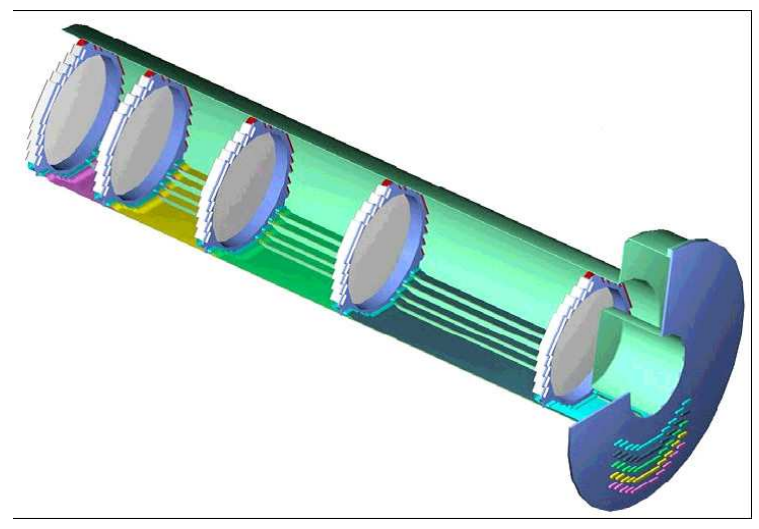

Figure 3.8: Engineering representation of 5 station fiber tracker

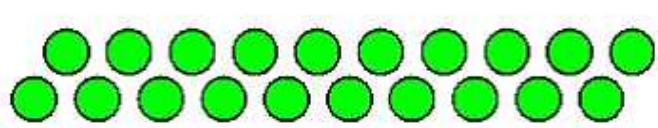

a)

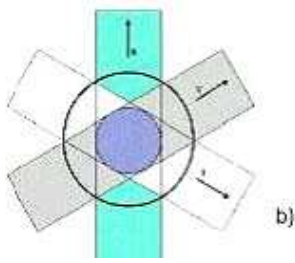

b)

Figure 3.9: a) Ribbon doublet structure; b) u-v-t fiber plane

Figure /reffig:8.1 illustrates the mechanical arrangement of one tracker. The five planes will be supported in a rigid tube so that the whole assembly can be slid into (or out of) the spectrometer solenoid. Two five-plane scintillating fibre trackers are envisioned, each plane consisting of three sets of fibre doublets at $120^{\circ}$ to each other (Figure 3.9). This structure will give efficient spacepoint reconstruction, since hits in any two of the three doublets suffice. Each doublet consists 
of two overlapping layers of round fibres, giving $100 \%$ geometrical coverage over the face of the detector and an approximately uniform thickness of scintillator, independent of where a particle crosses the doublet. This structure also suppresses the effects of background photons since a photon interaction is not likely to produce a triplet hit in a fiber plane. Each detector plane will have an active area that completely encloses a $30-\mathrm{cm}$-diameter circle. Figure shows the fibre ribbon doublet structure and a typical $\mathrm{u}-\mathrm{v}$-t readout plane. Scintillation light from the fibre will be piped to the photodetectors using clear fibre light guides of a length not to exceed $3 \mathrm{~m}$. The photodetector of choice is the Visible Light Photon Counter (VLPC), due to its high quantum efficiency (85\%) and high gain $(50,000)$. This design is inspired by that of the D0 Fibre Trackers [34] VLPC system. To minimize the effects of the background x-rays, the smallest fiber that yields enough light for efficient tracking will be used. Based on results from D0, $350 \mu \mathrm{m}$ fibre diameter seems acceptable, given the short active length $(30 \mathrm{~cm})$ of the MICE tracking planes and the relatively short light guides in comparison to those used in the D0 Fibre Tracker. Fibre ribbons made with both 500 and $350 \mu \mathrm{m}$ fibre are currently being prototyped. The light yield from these prototypes will be measured with a 32 channel VLPC system using cosmic rays.

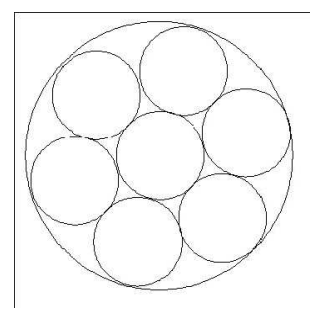

Figure 3.10: Fiber multiplexing scheme

\subsubsection{Readout electronics}

The readout planned for MICE uses the D0 VLPC system. A total of 44 D0-type 1024-channel VLPC cassettes are required. No new engineering is needed and the available infrastructure at Fermilab can be used for VLPC cassette production. A new order for VLPCs will have to be initiated with the vendor, however. Also, the D0 cryogenics design will be used for MICE. The current design calls for two D0-type VLPC cryostats each holding approximately 22,000 channels (22 cassettes) of VLPC readout. One cryostat will service the upstream tracker and one the downstream tracker. This configuration allows minimization of the fiber light guide length, thus maximizing light yield and reducing fiber costs. Each VLPC cassette uses two 512-channel readout boards. The existing boards currently used in the D0 experiment provide analog and trigger bit (digital) information from the VLPCs, temperature and bias control, and calibration. D0 is currently working on a new front-end design that will allow for higher readout rate and include the possibility of adding timing information (with 1-2 ns precision) for each hit. The MICE collaboration plan to work with D0 on this effort, with the hope that a single final design can be used for both experiments.

\subsubsection{TPG for the Optional Tracker}

This detector has been proposed [Gas02] with the acronym TPG (Time Projection chamber with GEM amplification [GDD02]). The ionization electrons generated by the muon in a gas volume 1 
$\mathrm{m}$ long and $30 \mathrm{~cm}$ in diameter will be drifted along an electric field parallel to the magnetic field, and multiplied by a factor 104 to 105 in a set of three gaseous electron multipliers (GEMs). (A GEM [Sau97] is a structure in which high gas gain results from the concentration of electric field at the edges of small holes bored in a thin double-clad plastic foil, across which a high voltage is applied.) The readout plane, called a hexaboard [Bre98, Sau01, Bac02], is made of hexagonal pads arranged to form three sets of strips oriented at $120^{\circ}$ to each other.

\subsection{DAQ and slow control}

\subsubsection{DATE}

Data Acquisition Test Environment (DATE) developed by the ALICE Collaboration have been chosen for the use in the MICE DAQ. Figure xxx show the architecture of DATE.

\subsubsection{EPICS}

EPICS provides a set of softwares for use in building distributed control systems to operate devices such as Particle Accelerators, Large Experiments and major Telescopes. Such distributed control systems typically comprise tens or even hundreds of computers, networked together to allow communication between them and to provide control and feedback of the various parts of the device from a central control room, or even remotely over the internet. EPICS uses client/server and publish/subscribe techniques to communicate between the various computers. Most servers (called Input/Output Controllers or IOCs) perform real-world I/O and local control tasks, and publish this information, which is defined as the Process Variable (PV) to clients using the Channel Access (CA) network protocol.

\subsection{MICE time development of apparatus}

Given that all detectors and parts of the equipment will not be ready at the same time, one can foresee a development of the experiment in time, to allow a number of preparatory stages. This leads to the scenario presented in Figure 3.11. First (step I) the beam can be tuned and characterized using a set of TOF and particle ID detectors. In step II the first spectrometer solenoid allows a first measurement of $6 \mathrm{D}$ emittance with high precision and comparison with the beam simulation. This should allow a systematic study of the tracker performance. Step III is fundamental for the understanding of a broad class of systematic errors in MICE. The two spectrometers work together without any cooling device in between and should measure the same emittance value (up to the small predicted bias due to scattering in the spectrometer material). Step IV, with one focusing pair between the two spectrometers, should give a first experience with the operation of the absorber and a precise understanding of energy loss and multiple scattering in it. Several experiments with varying beta-functions and momentum can be performed with observation of cooling in normalized emittance. Starting from step V, the real goal of MICE, which is to establish the performance of a realistic cooling channel. 


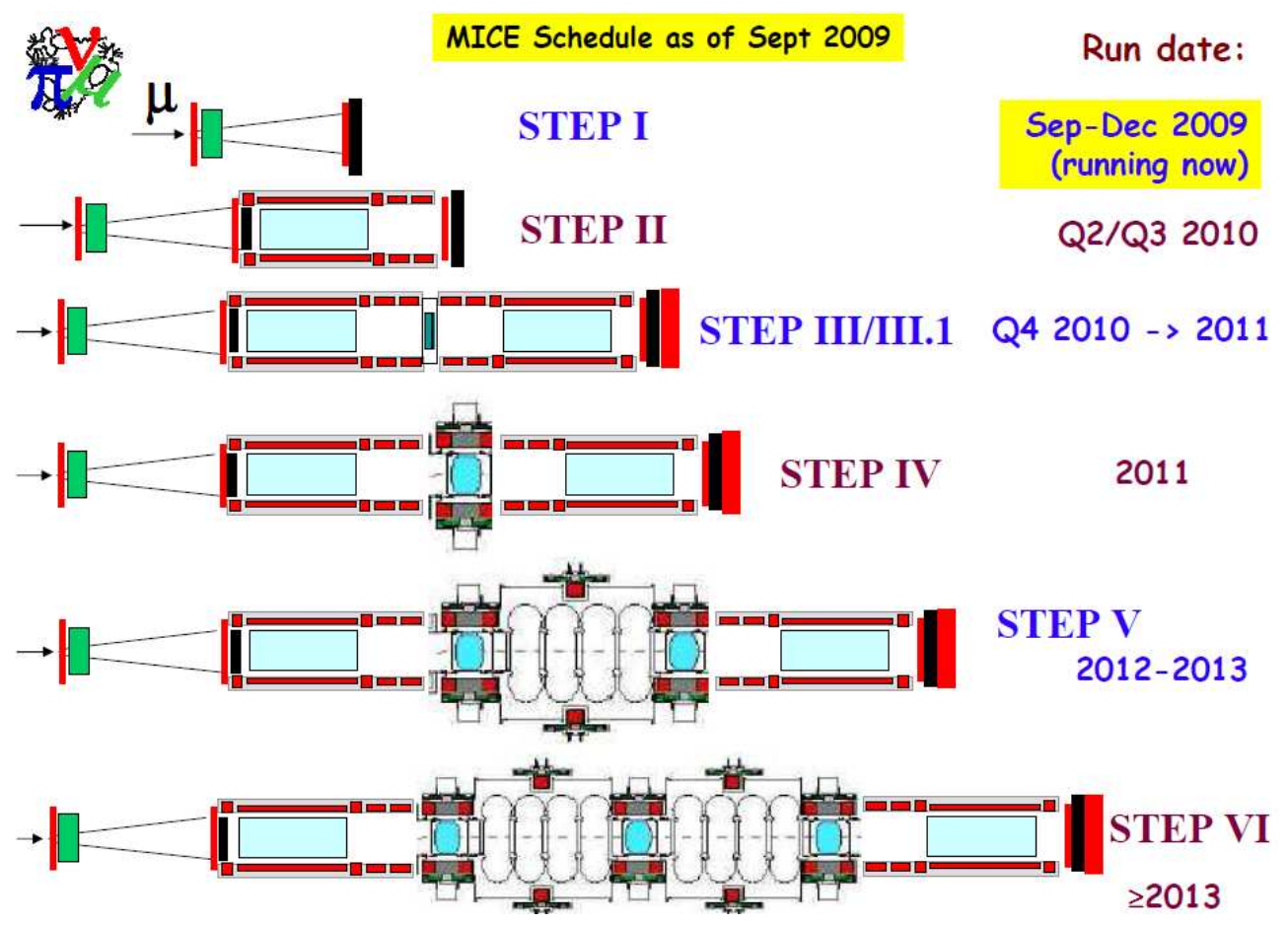

Figure 3.11: Evolution of the apparatus in time. 


\section{Chapter 4}

\section{Overview of the MICE Scintillating Fiber Tracker}

The MICE Scifillating Fiber Tracker (SciFi Tracker) has been developed for measuring muon emittance in MICE. The emittance measurements with high resolution requires the Tracker to be made with small amount of material for minimizing the effect of multiple scattering.

\subsection{Overview}

\subsubsection{Requirements}

First requirement for the MICE SciFi tracker is to be made with small amount of materials to reduce multiple scattering in order to achieve the emittance measurement with precision of \pm 0.1 \%. Next requirement for the tracker is the stable operation near high magnetic filed of 4 Tesla. MICE SciFi tracker has been designed to meet these two requirements as describe in the next.

\subsubsection{Key challenges}

In order to minimize the effects of multiple scattering, the SciFi Tracker consists of fine scintillating fibers of $350 \mu \mathrm{m}$ diameter. Photon detectors with high quantum efficiency are required in order to detect the low light produced by such a fine fiber. The Visible Light Photon Counter (VLPC) is employed for the SciFi Tracker. The VLPC peak quantum efficiency is more than $85 \%$ at 520 $\mathrm{nm}$ (green) wavelength. Hence, a 3HF (3-Hydroxyflavone) secondary dopant, with peak emission at $520 \mathrm{~nm}$, is employed. The scintillating fibers in which $3 \mathrm{HFs}$ are doped by $2500 \mathrm{ppm}$. The concentration of $3 \mathrm{HF}$ has determined by the result of cosmic-ray test. The details are explained in the section 5.3.3.

Clear-fiber light-guides transport scintillation photons to the VLPCs. In order to minimize the light yield loss in propagation, clear fibers with long attenuation length are employed for lightguides. The light-yield loss of about $50 \%$ is expected during the transportation in the light-guide of $4 \mathrm{~m}$ long. The attenuation length measurement is described in section 5.2.2.

The number of scintillating fibers in two Trackers reaches to 44,800. Seven of scintillating fibers are multiplexed to reduce the number of channels to 6,400. This degrades the position resolution by factor 4 , but it still requires the resolution of emittance measurement. 
Analog Front End Boards with version II (AFEIIt) have been developed for the use in MICE. The charges from VLPCs are digitized at ADCs and transported to FPGAs with time information. FPGAs packs the data (charge and time) in each channels into event fragments and transports those to VME memory buffer modules. As the memory buffer module, VME Surdes Board (VLSB) is used, which have been developed by FNAL. The brief description of AFEIIt and VLSB are presented in the section 4.3.2. At each ends of the spill, data stored in VLSBs are read out by DAQ.

MICE have been decided to use the DAQ framework, Data Test Environment (DATE), which has been developing by ALICE collaborators. The tracker DAQ have been implemented and tested in the cosmic-ray test at RAL in 2008. And also, EPICS based slow control has been developed for the purpose of initializing AFEIIt boards. The implementation of the tracker DAQ is described in section 7.2.4.

\subsection{SciFi tracker system}

\subsubsection{Layout of the system}

The SciFi tracker system is constructed from three sections; the SciFi tracker, light-guide and photon detecting system. Key parameters of the MICE tracker are summarized in Figure 4.1.

\subsubsection{SciFi tracker}

\subsubsection{SciFi ribbon}

The scintillating fibers of $350 \mu \mathrm{m}$ diameter are glued with $427 \mu \mathrm{m}$ pitch to make a single layer of the ribbon. A second layer of the ribbon is glued on the first layer in order to reduce the dead space between fibers. The ribbons are made in the FNAL following the technique developed by the D0 fiber tracker.

\subsubsection{SciFi station}

The SciFi station is the place where hit positions of muons are detected at the given z position in the tracker. In the station, three scifi ribbons (doublets) are glued with the 120 degree angular spacing on the station frame made by carbon fiber. The free fibers are bent to connect with optical connectors which are located on the circumference of the frame. There are 30 connectors in a station, six of which are connected to the 'internal' light-guide, therefore five light-guides are attached to a station. The optical connectors used for the station are shown in Figure 4.3. Five stations are fixed with the carbon-fiber space frame at the given location as shown in Figure xxx. The space frame with stations mounted are installed in the bore of the spectrometer magnet. In the cosmic-ray test, stations are installed in the light-tight cylindrical tube.

\subsubsection{Station support frame}

Station support frames are used to fix the SciFi stations at given z positions vertically to the beam axis. 


\begin{tabular}{|c|c|c|}
\hline Component & Parameter & Value \\
\hline $\begin{array}{l}\text { Scintillating fibre } \\
\text { tracker }\end{array}$ & $\begin{array}{l}\text { Scintillating fibre diameter } \\
\text { Primary dopant, pT, concentration } \\
\text { Secondary dopant, } 3 \mathrm{HF} \text {, concentration } \\
\text { Fibre pitch } \\
\text { Estimated light yield per single fibre } \\
\text { Number of scintillating fibres per } \\
\text { optical readout channel } \\
\text { Position resolution per plane } \\
\text { Views per station } \\
\text { Radiation length per station } \\
\text { Stations per spectrometer } \\
\text { Station separation: } 1-2 \\
\text { Station separation: } 2-3 \\
\text { Station separation: } 3-4 \\
\text { Station separation: } 4-5\end{array}$ & $\begin{array}{l}350 \mu \mathrm{m} \\
1.25 \% \text { (by weight) } \\
0.25 \% \text { (by weight) } \\
427 \mu \mathrm{m} \\
10 \text { photo-electrons } \\
7 \\
470 \mu \mathrm{m} \\
3 \\
0.45 \% \text { radiation lengths } \\
5 \\
35 \mathrm{~cm} \\
30 \mathrm{~cm} \\
25 \mathrm{~cm} \\
20 \mathrm{~cm}\end{array}$ \\
\hline Tracking volume & $\begin{array}{l}\text { Sensitive volume: length } \\
\text { Sensitive volume: diameter } \\
\text { Gas in warm bore of spectrometer } \\
\text { solenoid }\end{array}$ & $\begin{array}{l}110 \mathrm{~cm} \\
30 \mathrm{~cm} \\
\text { Helium at atmospheric } \\
\text { pressure }\end{array}$ \\
\hline $\begin{array}{l}\text { Spectrometer } \\
\text { solenoid }\end{array}$ & $\begin{array}{l}\text { Magnetic field in tracking volume } \\
\text { Field uniformity in tracking volume } \\
\text { Field stability } \\
\text { Warm bore diameter }\end{array}$ & $\begin{array}{l}4 \mathrm{~T} \\
3 \text { per mil } \\
1 \text { per mil } \\
40 \mathrm{~cm}\end{array}$ \\
\hline
\end{tabular}

Figure 4.1: Key parameters of the MICE tracker module. The first section of the figure presents the parameters of the scintillating fiber tracker itself. The second section reports the environment within the tracking volume in which the tracker must operate. The final section reports the parameters of the MICE spectrometer solenoid that directly affect the performance of the tracker. 


\subsubsection{Light-guide}

Kuraray, clear polystyrene, round, s-type, $1.05 \mathrm{~mm}$ fiber was chosen for the light-guides. The attenuation length of the fiber was measured to be $7.6 \mathrm{~m}$ (section 5.2.2) and thus, to reduce the light loss in propagation as far as possible, the length of the clear-fiber run from station to VLPC cassette was specified to be $4 \mathrm{~m}$. The light-guide, a bundle of 128 clear fibers, is composed of an 'internal' section, which connects between the station and the patch panel, and 'external' section, which connects between the patch panel and the VLPC cassette. In order to make the cabling of the 'internal' light-guide to be clear, the length of the 'internal' light-guide is determined by the distance between the patch panel and the station where light-guide is attached. The length of (internal) clear fiber is determined by the distance between the patch panel and the station to be connected. This makes it possible to attach light-guide near the station support frame without making extra bending. The specification of the length of light-guides produced are summarized in Table 6.2. Optical grease are applied to all optical connectors when mating the light-guides in order to minimize light yield loss due to Fresnel reflection.
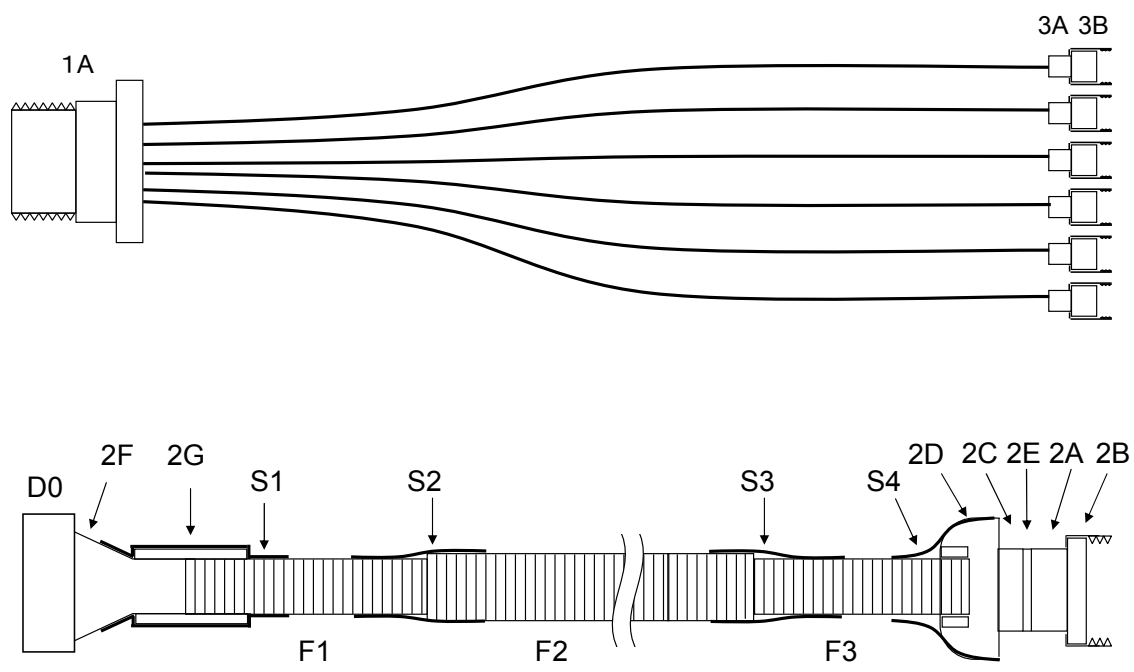

Figure 4.2: Schematic drawings of internal light-guide (top) and external light-guides (bottom).

\subsubsection{Internal light-guide}

The internal light-guide (figure 17 top) has one 128-fiber 'patch-panel' connector (1A) and six 22 -fiber station connectors (3A). The connector 1A (figure 18 top-left) has 128 holes which match with those of the connector $2 \mathrm{~A}$ (figure 18 bottom-right) of the external light-guide. The connector $3 \mathrm{~A}$ (figure 18 top-right) has 22 holes configured as shown. These connect to station connectors and are locked by the collar 3B. 


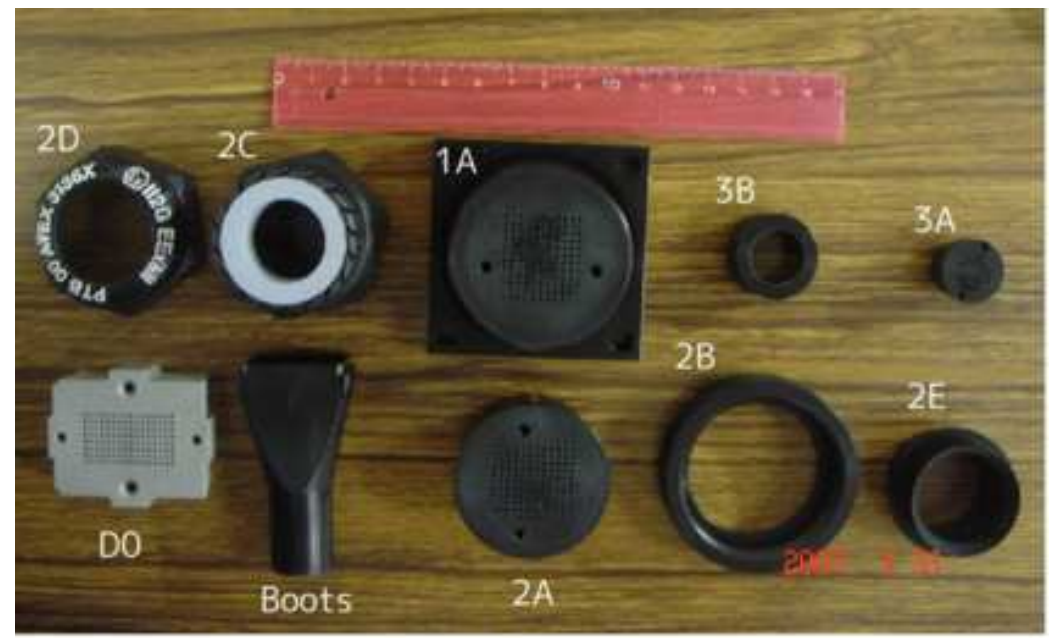

Figure 4.3: Optical connectors for SciFi station and light-guides.
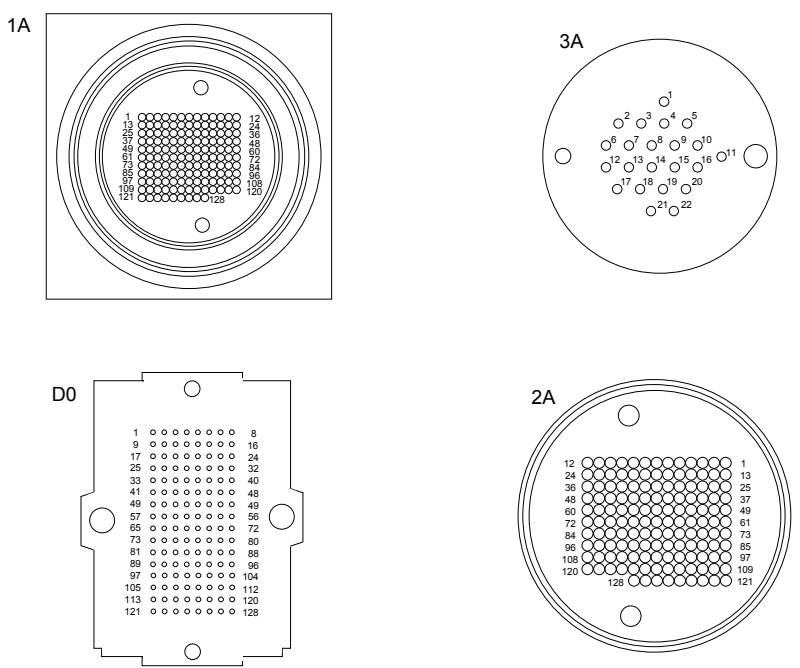

Figure 4.4: Channel assignment at optical connectors. 


\subsubsection{External light-guide}

The external light-guide is equipped with a flexible tube which shields the fibers from ambient light and also protects the fibers from any damage during handling and assembly. The external light-guides (figure 17 bottom) use a 128-fiber optical connector, developed by D0, at the end that interfaces to the VLPC cassette and a connector assembly (2A-E) which mates to connector 1A of the internal light-guide. Flexible tubes F1, F2, and F3, and heat shrink tubes S1, S2, S3, and S4 form the body casing of the external light-guide. A light-tight injection-moulded boot $(2 \mathrm{~F})$ is glued into the rear of the D0 connector and flexible tube F1 is attached to the boot using sleeve $2 \mathrm{G}$ with adhesive and heat-shrink tubing S1. This makes the assembly fully light tight and adds strength. The flexible tubes F1, F2, and F3 are fixed with heat-shrink tubes S2 and S3. The locking ring $2 \mathrm{~B}$ is used for securing the connector, $2 \mathrm{~A}$ to the connector $1 \mathrm{~A}$ of internal light-guide at the patch panel. The optical connector D0 (figure 18 bottom-left) has 128 holes, in a 16 x 8 arrangement which makes contact with the connector at the VLPC cassette. The optical connector 2A (figure 18 bottom-right) has 128 holes in an arrangement as shown, which mates with connector $1 \mathrm{~A}$ of the internal light-guide.

\subsubsection{Patch panel}

Patch panel is a aluminum plate at which internal light-guides and external light-guides are joined. 25 holes are made on the circumstance of the panel to fit the bulk head connectors of internal light-guides.

\subsubsection{Photon detecting system}

\subsubsection{VLPC}

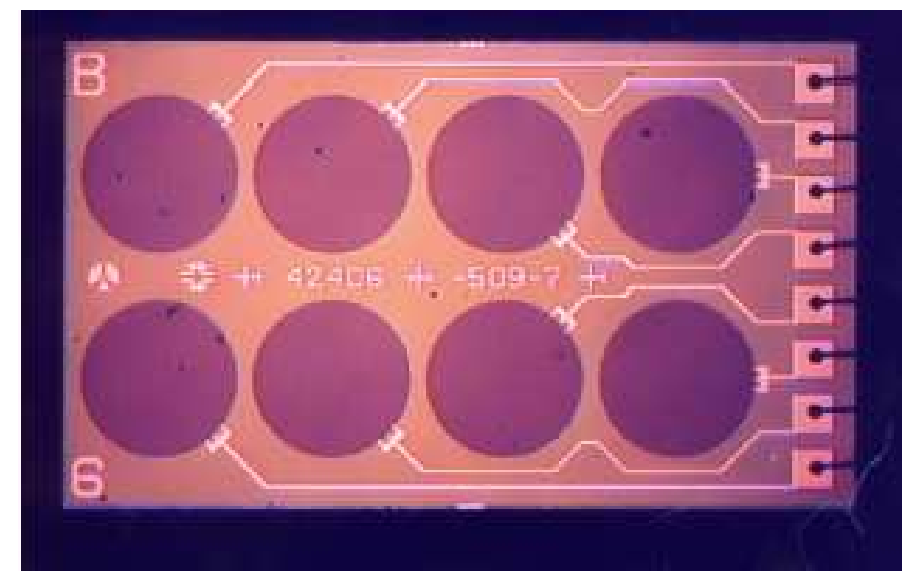

Figure 4.5: Photograph of the 8-element VLPC array.

The light produced from charged particles passing through the scintillating fibers is piped along the fibers and thence, via the clear-fiber light-guides, to the VLPCs, which are housed in cassettes (see below) and which convert the light into an electrical signal. The VLPC is a silicon-avalanche device operated at cryogenic temperature. It is a development of the Solid State Photomultiplier, an impurity-band silicon avalanche photo-detector. It has undergone six design iterations, specified 

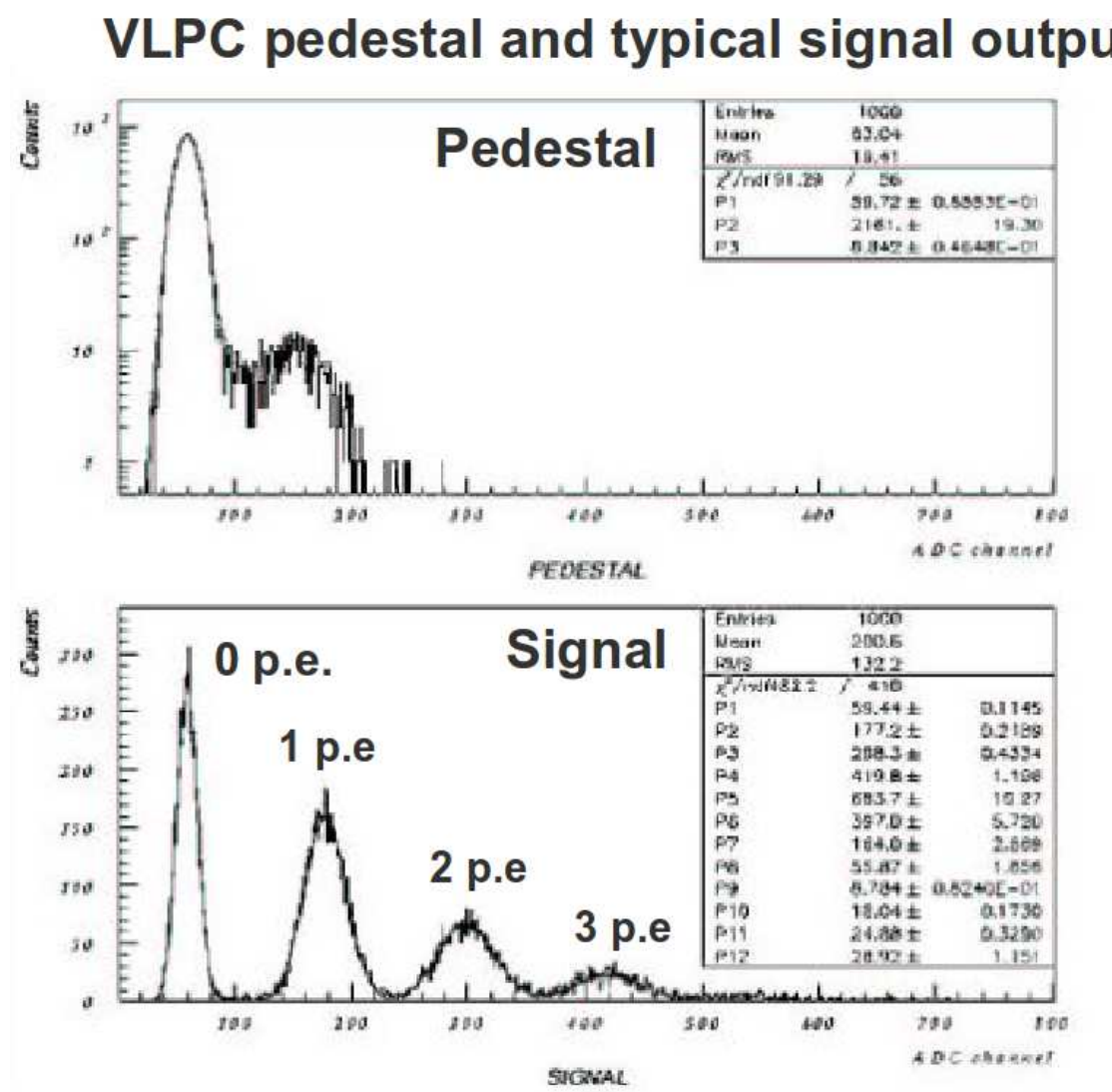

Figure 4.6: VLPC pedestal and typical signal distributions. One photo-electron noise of order $10^{-2}$ can be observed. Photon peaks are separately seen. 
as HISTE I to HISTE VI. HISTE VI, the version used in the D0 CFT, and thus the version that is used in MICE, is an eight element array in a 2-by-4 geometry, figure 29. Each pixel in the array having a diameter of $1 \mathrm{~mm}$. The HISTE VI operational parameters are: quantum yield, $>0.75$; gain (G), 20,000 - 60,000; operating temperature; $9 \mathrm{~K}$, and operating bias, $68 \mathrm{~V}$. VLPCs are fabricated using an epitaxial growth process which produces a series of doped and undoped silicon layers on highly-doped silicon substrate wafers. Each eight-pixel chip (Figure 4.5) is soldered to an aluminium nitride substrate. The outputs from individual pixels are wirebonded to individual contact pads on the substrate. VLPCs from different batches exhibited a large variation in gain, due to non-uniformities in the production process. Due to these variations, the VLPCs were sorted into like-gain batches which were used for specific cassettes. VLPC cassettes can be roughly characterized as high-gain ( $\mathrm{G} \tilde{4} 0000)$ or low-gain (G $\tilde{2} 0$ 000). MICE will use both high and low-gain cassettes.

\subsubsection{VLPC cassette}

The VLPC cassette contains 1024 channels of VLPC readout and is divided into 8 modules of 128 channels each. Sitting directly over each VLPC pixel is an optical fiber which brings the light from the detector to the VLPC chip. Each cassette module is composed of an optical bundle assembly, a cold-end electronics assembly, and an assembly of mounted VLPC hybrids.

The cassette has a 'cold end', that portion of the cassette which lies within the cryostat, and a 'warm end', the portion of the cassette which emerges from the cryostat and is at room temperature. At the cold end, eight 'cold-end assemblies', each of 128 channels of VLPC readout, are hung from the feed-through by the optical bundles and are surrounded by a copper cup. Each cold-end assembly consists of sixteen, 8-channel VLPC hybrid assemblies, the 'isotherm' or base upon which they sit, the heater resistors, a temperature measurement resistor, coldend flex-circuit connectors and the required springs, fasteners and hardware. Running within the cassette body from top to bottom are eight 128-channel optical-bundle assemblies which accept light from the detector lightguides connected to the warm-end optical connectors at the top of the cassette and pipe the light to the VLPCs mounted at the cold end. The electronic read-out boards are located in rails which are mounted on the warm end structure and are connected electrically with the cold-end assemblies via kapton 'flex circuit'. In addition, the electronics boards are connected to a backplane card and a backplane support structure via multi-pin connectors and board-mount rails. The flex circuits and read-out boards are electrically and mechanically connected by a high density connector assembly.

Since the VLPCs operate at cryogenic temperatures, a helium cryo-system is required. A special purpose cryostat has been designed and built for MICE to allow the VLPCs to be operated at a temperature of $\left(\begin{array}{ll}9.0 & 0.1\end{array}\right)$. The cryostat has two cassette slots which accept D0 1024 channel VLPC cassettes. The MICE VLPC cryo-systems use Gifford-McMahon cryocoolers (Sumitomo RDK 415D) to maintain the $9 \mathrm{~K}$ operating temperature for the VLPCs. This two-stage, commercial

cryocooler is located between the two cassette slots. The first stage of the cryocooler, operating at $50 \mathrm{~K}$, is used to provide a heat intercept between the room-temperature cryostat body and the cold-end assembly. The cold head of the cryo cooler is used to cool the copper isotherm in order to bring the VLPCs to their operating temperature.

The cassette envelope is a low conductivity, low thermal-expansion box that seals to the underside of the top lid of the cryostat. The insulating vacuum is on the outside of the envelope and the VLPC cassette, immersed in helium gas, is situated inside the envelope. The nominal clearance inside the cassette is made rather tight at $0.6 \mathrm{~mm}$ in order to facilitate heat conduction 
to the cryocooler. The wall thickness, $0.38 \mathrm{~mm}$, was also reduced to the minimum practical for fabrication so as to minimize heat conduction along the cassette envelope from the warm end to the cold end.

Thermal links provide the conduction heat-transfer path between the two stages of the cryocooler and the cassette. The thermal links are made from oxygen-free high-purity copper (OFHC), UNS grade C10100. The thermal links are $10 \mathrm{~mm}$ thick solid copper pieces with a short flexible segment to accommodate a movement of $2 \mathrm{~mm}$ due to thermal contraction. The flexible segment is constructed of 35 individual $0.13 \mathrm{~mm}$ thick pieces of high purity copper foil soldered to the solid segments. The upper thermal link operates at a temperature of around $4550 \mathrm{~K}$ and has a temperature gradient of $1 \mathrm{~K}$ from the cryocooler connection to the envelope connection. The lower thermal link (Figure 4.7) operates at temperatures in the range of $68 \mathrm{~K}$ and has a calculated 0.1 $\mathrm{K}$ temperature drop from the cryocooler to the envelope.

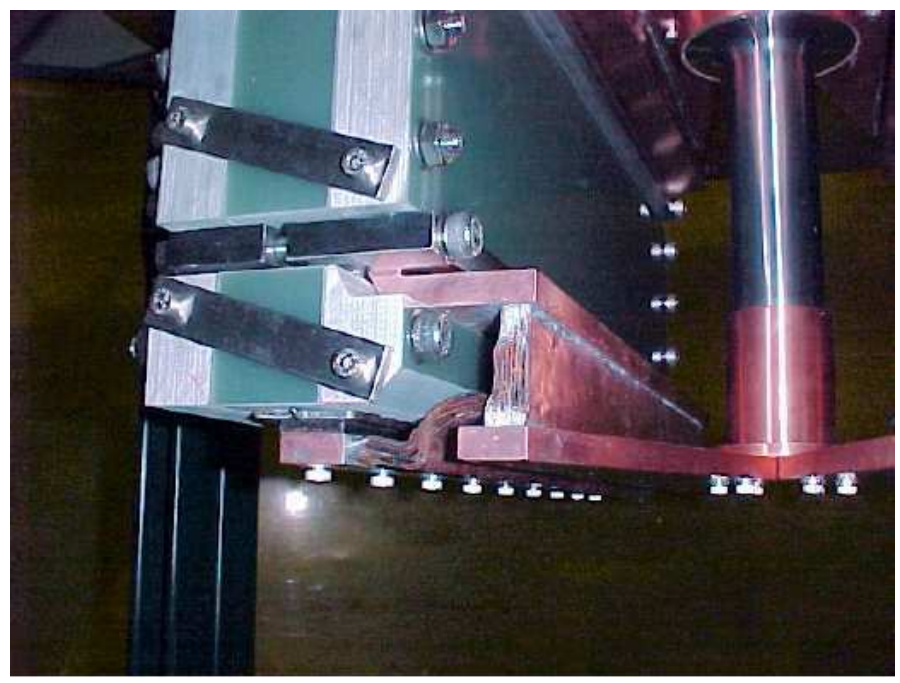

Figure 4.7: Close up of the lower thermal link flexible section. The cassette envelope may be seen on the left-hand side of the picture. The copper foils that form the thermal link between the cold end and the cryocooler cold head may also be seen. The Cernox temperature sensor is mounted to the envelope side of the link.

\subsubsection{VLPC cryostat with cryocooler}

Cernox temperature sensors from Lakeshore Cryogenics are mounted on the cold head of the cryocooler and the lower thermal link near the cassette envelope. A silicon diode temperature sensor from Oxford Instruments is used to measure the upper link temperature. A 100 platinum resistor was also used during the commissioning tests. The sensors are mounted into small copper holders using GE-7031 varnish. The copper holders themselves are screwed to the surfaces with a \#4-40 screw with Apiezon ' $\mathrm{N}$ ' grease at the interface. Cryogenic quad-lead wire from the sensors is heat sinked to a small copper bobbin mounted nearby. Readout and temperature control is via an Oxford Instruments, ITC503 temperature controller. A flexible 36 heater element is wrapped around the second stage of the cryocooler using high purity copper sheeting and hose clamps. The heat transfer surface of the heater is lightly coated with Apiezon grease. Up to $5 \mathrm{~W}$ of heat can be 


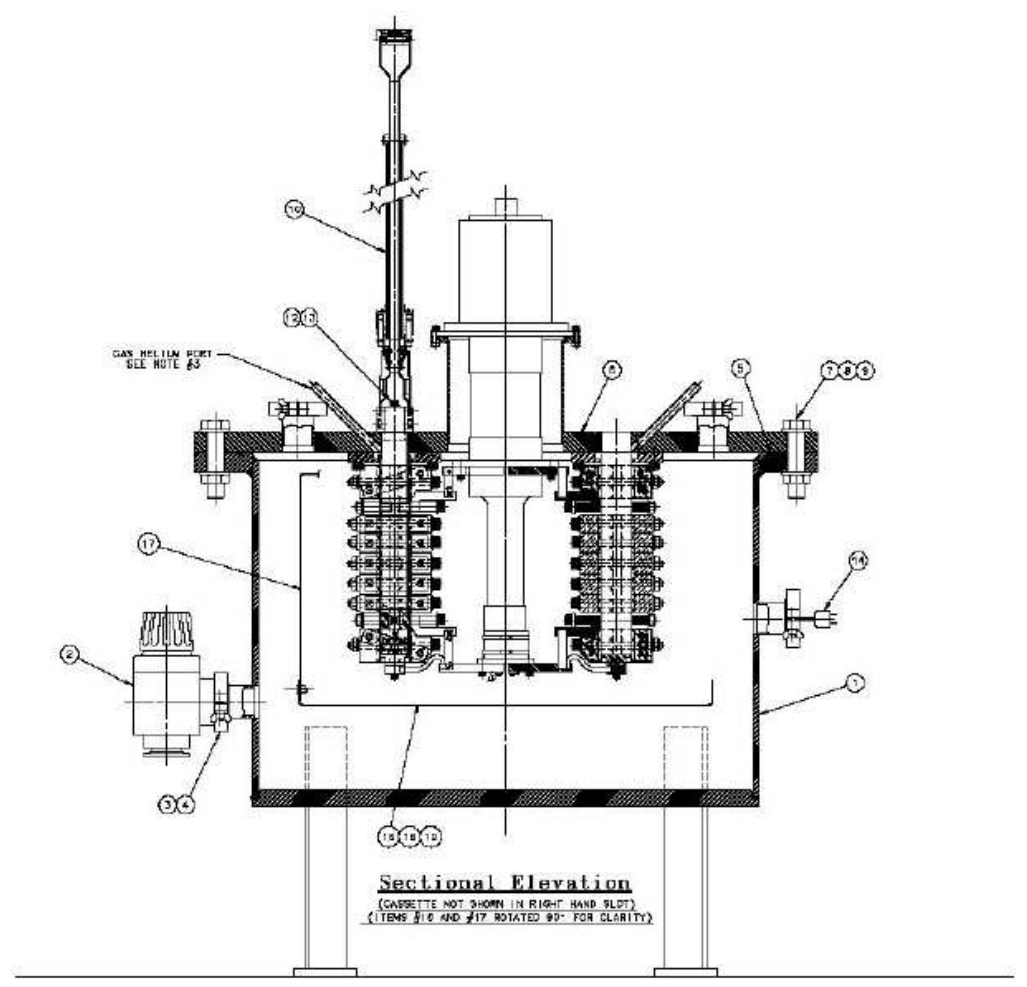

Figure 4.8: Sectional elevation drawing for the MICE 2-cassette VLPC cryostat

supplied by the heater to give gross temperature control for the cassette cold end. Each cassette also has individual heater controls (one heater for each module in a cassette for a total of 8) to adjust for small, asymmetric temperature differences.

Although we experienced some difficulty in making good (high-conductance) connections on the thermal links at the cryocooler head and the cassette envelope, once good link connections had been made, the system performed extremely well. In normal operating conditions, a small amount of heat is added to stage II using the heater (supplied and controlled by the Oxford controller) giving better temperature stability. With full control (Oxford and cassette temperature control on), the VLPC temperature is controlled at $(9 \pm 0.0005) \mathrm{K}$.

\subsection{Tracker Readout}

\subsubsection{Trigger logic}

In MICE, the L1ACCEPT will be formed from signals from Time-of-Flight counters and signals from the ISIS accelerator; a combination of these signals will indicate the passage of a throughgoing muon. If we assume a $0.5 \%$ occupancy, then there are roughly 30 hits in the two trackers per muon. During readout, all 64 DFPGAs will read out a trigger word, a header word, and 8 words for bitmap data for a total of 10 words per DFPGA. Then 30 single hits with 5 words per hit (channel, TriP0 time, TriP1 time, TriP0 charge, and TriP1 charge) yield 150 more words for a total of (64 $\mathrm{x} 10)+(30 \times 5)=790$ total words for a MICE event. Assuming 1000 words/event, then for 600 events/spill and one spill every second, the AFE-IIt boards will have to read out roughly 600,000 
words every second to the VLSB buffers via the LVDS links and VME. If the data are read out to 16 VLSB modules housed in two crates with each VME word transfer taking $1 \mu \mathrm{s}$, the 600,000 word readout would take roughly $0.3 \mathrm{sec}$ which is sufficient for MICE running.

\subsubsection{Electronics}

\subsubsection{AFEIIt board}

The board that will be used in MICE is the second generation front-end readout board, the AFEIIt [14]. Figure 35 shows the schematic diagram of signal pathes from VLPCs to LVDSs (128 channels) implemented on the AFEIIt board. Four of this diagram are implemented in a board, therefore, the number of channels per board is 512. To read out two trackers, 6,400 channels are needed, so that 13 AFEIIt boards are required in theory. Since light-guides for upstream(downstream) tracker cannot reach to VLPC cryostats at the downstream(upstream), 16 AFEIIt boards are required in realily.

As shown in figure 35, the analogue pulses produced by the VLPCs are input to 'Trigger and Pipeline with timing' (TriP-t) chips on the AFE-IIt boards. The TriP-t chip generates three outputs for each channel: a digital discriminator signal; an analogue pulse proportional to the amplitude of the integrated charge of the input pulse (the A-pulse); and an analogue pulse (the t-pulse) proportional to the time between the firing of the discriminator and the closing of the time gate. The discriminator output is routed to one of the Field Programmable Gate Arrays (FPGAs), the DFPGA ('Digital' or 'Discriminator'). The A-pulse and the t-pulse are stored in 48-sample, analogue pipelines in the TriP-t chip before being read out upon the receipt of an external Level 1 accept (L1ACCEPT) trigger. The discriminator bits, which indicate which channels are above a predetermined threshold, will be directed to another FPGA called the AFPGA ('Analogue'), and will be used for the digitization of the data in the channels above threshold. Only those channels above threshold are digitized ('zero-suppression'), which results in reducing digitization time.

The AFEIIt boards are initialized before starting DAQ, in which TriP-t and FPGAs are turned on and configured. As noted in section xxx, the temperature and heater power at cassettes can be read out via the right-hand AFEIIt board. This monitoring performs at every time after Oxford temperature controller is turned on to confirm that the temperature of cassette reaches at $9 \mathrm{~K}$ stably. The initialization and monitoring is controlled by EPICS (section 7.2.3).

\subsubsection{VLSB slave board}

A VME LVDS SERDES (Serializer/Deserializer) Buffer (VLSB) board has four memory buffers (banks), each of them is connected to a AFEIIt board via a Low Voltage Differential Signal (LVDS) cable. The information on A-pulse (ADC) and t-pulse (TDC) from Trip-t chips are packed into an event by FGPAs in AFEIIt board and read out over a LVDS cable upon receipt of a L1ACCEPT. Data stored in VLSB boards are read out at every end of the spill by the DAQ. Data format implemented in the DAQ is shown in Figure 7.12.

The VLSB module, which was originally designed for D0 board testing and has been adopted for use in MICE, is a VME64 single width, $6 \mathrm{U}$ module. The module is a custom LVDS SERDES buffer with 4 LVDS input channels and can be operated stand-alone. A VLSB module can receive/generate trigger signals over two LEMO connectors on the module front panel. A block diagram of the VLSB card is shown in figure 36 [22]. The module can be controlled through two different interfaces; RS232 (front panel) and VME (backplane). The VLSB module hosts the Module Controller FPGA 
which handles the VME and RS232 interfaces, the timing and the diagnostics, and supervises the data flow operations. The FPGA is configured at power-up by two on-board EEPROMs. The VLSB module also hosts the four LVDS SERDES receivers used to convert and de-serialize the input LVDS signals to Low Voltage TTL. A logic analyzer pod is provided for each LVDS link. The Module Controller FPGA manages the input LVDS interface and stores the data received into the ZBT SRAM. The FPGA control/status registers and the ZBT SRAM content can be accessed from both VME and RS232 interfaces.

The detail on the operation of the VLSB by the DAQ is described in section 7.2.4.

\subsubsection{DG2020/VLSB master board}

DG2020 function generator makes L1ACCEPT signals which is need as external trigger for AFEII board. The same functionality is implemented in a VLSB board (referred to as master board to distinguish memory buffer module). The timing configuration of the external trigger can be handled via VME access.

\subsubsection{MIL-1553 board}

The MIL-1553 board is the VME interface to the AFEIIt board, which is used when initializing AFEIIt boards or monitoring the cassette data such as the temperature and heater value. The MIL1553 board has two channels, each is connected with the back plane where four AFEIIt boards are connected and identified by the RT (Remote-Terminal). The command is transfered from one channel to one board at a time.

\subsubsection{Tracker DAQ}

The tracker DAQ has a responsibility to take tracker data stored in VLSB boards and transfer to the LDCs for recording locally or the GDC for event building after each end of the trigger. In the tracker DAQ, equipments of VLSB boards are defined for taking data and an equipment of the trigger counter is for managing a trigger. The DATE based DAQ has been implemented and tested in the cosmic-ray test at the RAL in 2008, In this test, data is transfered to the local LDC, while in the MICE physics run, tracker data will be transfered to the GDC. The implimention of the tracker DAQ is described in chapter 7.

\subsubsection{Tracker slow control}

The tasks of the tracker slow contorl are 1) initialization, which is required prior to starting the DAQ and 2) monitoring, which is requested when especially checking that cassettes are cooled stably. In order to inform results of the tasks to clients, following PVs are defined; the status flag which indicates that the initialization has done successfully and the cassette data such as temperature and heater value. The AFEIIt CA server starts the initialization (monitoring) of AFEIIt boards when requested by clients then updates PVs. The slow control for the tracker has implemented and tested in the cosmic-ray test at the RAL in 2008. In this test, the AFEIIt CA clients are implemented in the DATE functions; the initialization is in the Arm function of the AFEIIt board, the monitoring is in the Read function of the AFEIIt board. In the MICE physics run, the monitoring will be separated from the DAQ, that is, the cassette data will not be recorded in the GDC. The monitoring will be performed once after the cassette cooling will be performed 
in order to confirm the temperature will have reached down to $9 \mathrm{~K}$ stably. The implementation is described in section 7.2.3. 
40 CHAPTER 4. OVERVIEW OF THE MICE SCINTILLATING FIBER TRACKER 


\section{Chapter 5}

\section{SciFi Tracker Prototype}

The prototype tracker has been made to check the mechanical design and measure light-yields of scintillating fiber with clear-fiber light-guide attached at the cosmic-ray test.

The fiber selection procedure is applied both for the scintillating fiber and clear fiber. With respect to the scintillating fiber, attenuation length measurement (see Section 5.2.1) and the selection of $3 \mathrm{HF}$ concentration (see Section 5.3.3) have been performed. The clear-fibers have been determined in terms of the long attenuation length and high tolerance to the bending. The attenuation length measurement is described in section 5.2.2.

The prototype tracker contains only three stations, two of them have only two views due to the availability of scintillating fibers. The one of the most significant difference from the MICE tracker is the design of the optical connectors. This improvement allow the assembling to be easy.

The cosmic-ray test shows that the measured light-yield is 11 pe, which agrees with the expectation from data taken at D0 fiber tracker (see Section 5.3). After that, the tracker production has been started. (see chapter 6 )

The prototype has been also tested with muon beams at KEK in 2005, where the 1T superconducting magnet is employed. The cryocooler prototype is also tested in the test.

\subsection{Mechanical design for prototype}

\subsubsection{SciFi station}

Three SciFi stations are prepared for the test. Two station has two views and one station has three views. Each view is made with scintillating fiber with different concentration of $3 \mathrm{HF}$. The specification of the concentration in each view is summarized in Table xxx. Three stations are fixed using four carbon-fiber frames with equal spacing of $15 \mathrm{~cm}$. The tracker is installed in a light-tight tube of about $1 \mathrm{~m}$ height. The light-guides are extracted out of the tube through holes on a panel fixed on the top of the tube.

\subsubsection{Optical connector}

Two kinds of optical connectors are used for the prototype tracker. One is the MICE connector attached on the station, and the other is the D0 connector attached at the cryostat. The optical connector has 18 holes. The D0 connector has 128 holes (2 holes are not used). The optical connectors are aligned by dowel pins and contacted by optical grease. 


\subsubsection{Scintillating fiber}

The scintillating fiber prepared is Kuraray $350 \mu \mathrm{m}$ SCSF-3HF, ROUND, Multi-cladding, S-type with $1 \% \mathrm{pT}$. Multi-cladding is selected because of the higher trapping efficiency than singlecladding. Multi-cladding also has less effect of light-yield loss due to the crack on the surface than single-cladding. S-type has more tolerance to the bending than Non-S type, while the attenuation length of the former is shorter than the latter. In view of minimizing the risk of damaging the fiber in handling, S-type is selected.

The second dopant, $(3 \mathrm{HF})$ is mixed to shift the wavelength of the scintillating light down to $525 \mathrm{~nm}$ where the VLPC has highest quantum efficiency, $85 \%$. The emission spectrum of $3 \mathrm{HF}$ are shown in Figure5.1.

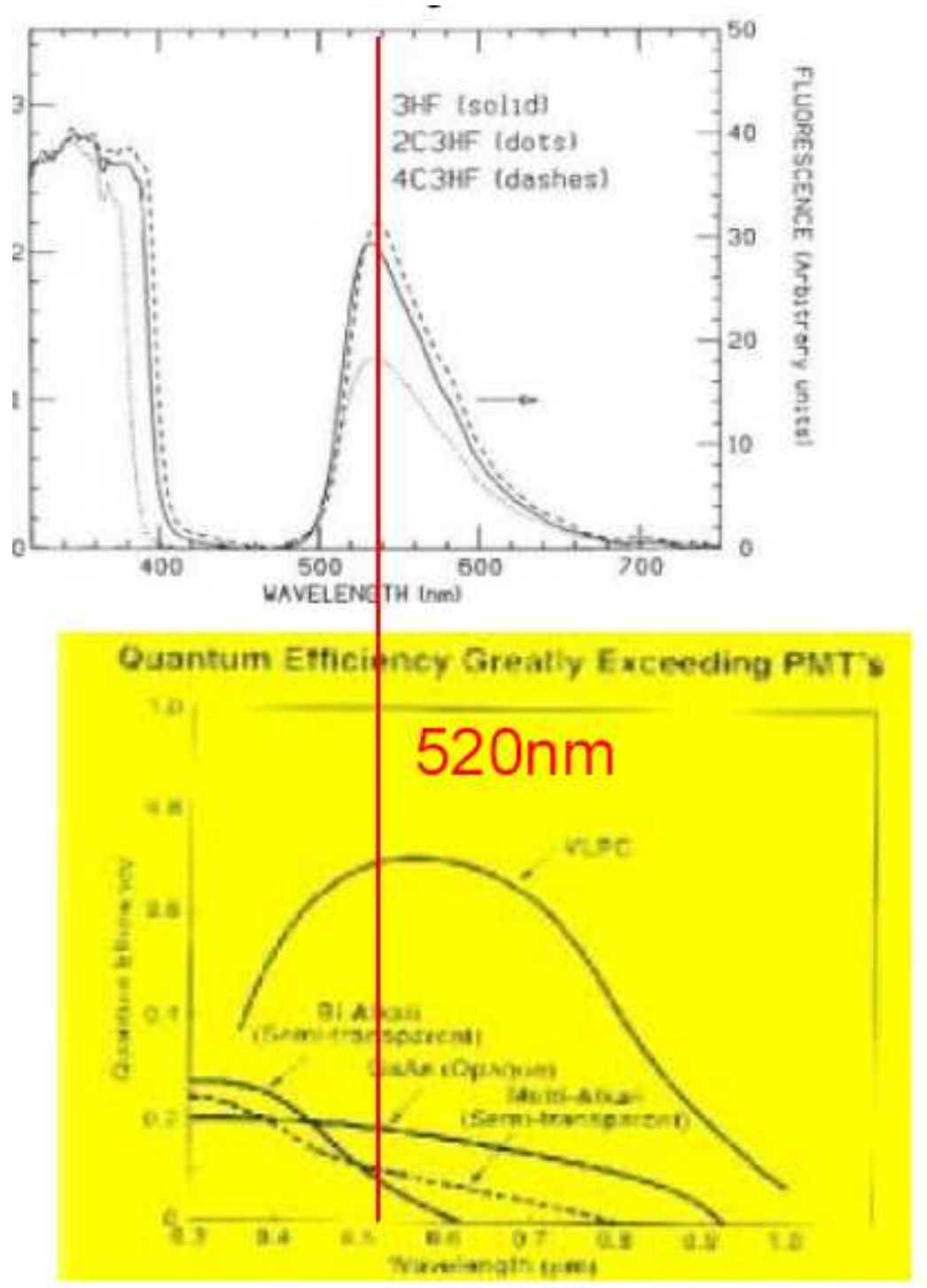

Figure 5.1: Emission spectrum of the second dopant, 3HF (top) and VLPC quantum efficiency curve (bottom). 


\begin{tabular}{|c|c|c|c|}
\hline 3HF $(\mathrm{ppm})$ & 10000 & 5000 & 3500 \\
\hline $\mathrm{A}$ & $399.7 \pm 15.5$ & $306.7 \pm 11.4$ & $239.7 \pm 12.4$ \\
\hline $\mathrm{a}(\mathrm{m})$ & $0.248 \pm 0.019$ & $0.369 \pm 0.031$ & $0.498 \pm 0.041$ \\
\hline B & $562.3 \pm 10.0$ & $545.1 \pm 12.2$ & $467.1 \pm 13.9$ \\
\hline $\mathrm{b}(\mathrm{m})$ & $2.10 \pm 0.04$ & $2.68 \pm 0.06$ & $2.60 \pm 0.07$ \\
\hline
\end{tabular}

Table 5.1: The attenuation length of scifi with several 3HF concentration. A and a are the magnitude and the attenuation length respectively, and $\mathrm{B}$ and $\mathrm{b}$ are these for the long component.

\subsubsection{Light-guide}

A light-guide is a bundle of 126 ( $7 \times 18)$ clear fibers of $4 \mathrm{~m}$ long. Seven MICE connectors at the end and one D0 connector are attached at the other end of light-guides. In order to shield the light from the outside, flexible tubes are attached from the outside of the patch panel to end of D0 connectors attached on the cryostat.

\subsection{Attenuation length measurement}

\subsubsection{Scintillating fiber}

Three kinds of concentrations, 3500ppm, 5000ppm and 10000ppm were tested.

A collimated light from a LED (NSHU-550A) of which emission peak is $375 \mathrm{~nm}$ was injected in order to excite the scifi. The measurement was performed with changing the lightning point from $10 \mathrm{~cm}$ up to $3 \mathrm{~m}$ from the size of the photo detector, PMT (H1949), by HAMAMATSU. The end of the scifi was polished briefly and fixed on a thin acryl board. The polished end was connected optically with the surface of the PMT cathode while the other end is free.

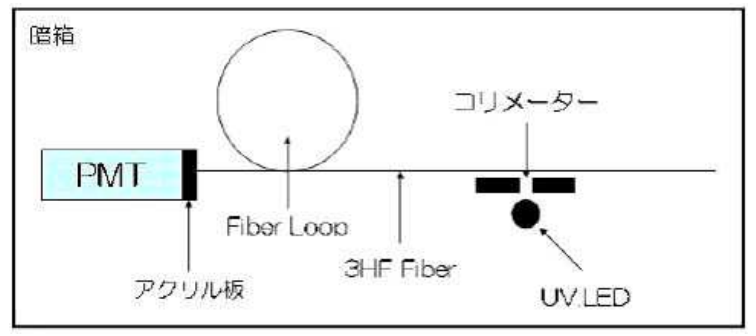

Figure 5.2: Layout of the attenuation measurement of scifi.

The attenuation length is estimated by fitting with the sum of two exponential functions as follows. As a fitting function, the summation of exponential function with short component $\left(\lambda_{S}\right)$ and long component $\left(\lambda_{L}\right)$ is applied,

$$
F(x)=A \times \exp \left(-x / \lambda_{S}\right)+B \times \exp \left(-x / \lambda_{L}\right)
$$

The result of the attenuation length is summarized in Table xxx. 


\begin{tabular}{|c|c|}
\hline $\mathrm{A}$ & $0.32 \pm 0.77$ \\
\hline$\lambda_{S}(\mathrm{~m})$ & $0.69 \pm 0.74$ \\
\hline $\mathrm{B}$ & $1.49 \pm 0.07$ \\
\hline$\lambda_{L}(\mathrm{~m})$ & $7.63 \pm 0.70$ \\
\hline
\end{tabular}

Table 5.2: The result of the measurement of clear fiber.

\begin{tabular}{|c|c||c|c|}
\hline $\mathrm{A}$ & $\lambda_{S}(\mathrm{~m})$ & $\mathrm{B}$ & $\lambda_{L}(\mathrm{~m})$ \\
\hline $0.32 \pm 0.77$ & $0.69 \pm 0.74$ & $1.49 \pm 0.07$ & $7.63 \pm 0.70$ \\
\hline
\end{tabular}

Table 5.3: The result of the measurement of the attenuation length of clear fiber.

\subsubsection{Clear fiber}

The clear fiber prepared is KURARAY $1.05 \mathrm{~mm} \phi$ CLEAR-PS, ROUND, S-type.

The attenuation measurement of the clear fiber, KURARAY CLEAR-PS, ROUND, S-type, 1.05 $\mathrm{mm} \phi$, was measured in the dark room at Lab6 in the FNAL. A schematic view of the setup is shown in Figure 5.3.

The light from scifi was used as source and two sets of silicon photo-diodes provide by Oriel were used as detector. The bundle of scifi was set in a plastic jointer. The clear fiber of a length of $8 \mathrm{~m}$ was then inserted in a hole of the jointer, so that the light from scifi can inject directly into the clear fiber. The jointer, a LED to excite scifi and the photo-detector to measure the amount of light from LED were fixed and mounted on a support stand. The LED was driven by a $100 \mathrm{~Hz}$ pulse generator with a $5.5 \mathrm{~V}$ pulse-height. The other end of clear fiber was inserted in a connector to set in a optical diffuser where the second photo-detector was attached. The diffuser was employed to reduce the directionality of the light. After the first measurement, the end of fiber set in diffuser was cut by $50 \mathrm{~cm}$ then polished with a portable diamond cutter. The measurement was repeated until the length of the clear fiber reached to $50 \mathrm{~cm}$.

The light-yield as a function of the length of the clear fiber is show in Figure 5.4. The attenuation length is estimated in the same way as scintillating fiber.

The result of the attenuation length measurement is summarized in Table 5.3. From this, $55 \%$ light yield loss is estimated with a $4 \mathrm{~m}$-long clear fiber.

\subsection{Cosmic-ray test at FNAL}

The light-yield of the prototype tracker with $4 \mathrm{~m}$ light-guide attached had been measured with cosmic-ray using VLPCs at the FNAL D0 test stand in 2003 (Figure 5.5).

\subsubsection{Setup}

\subsubsection{Prototype tracker}

The construction and assembling of the prototype tracker were performed at the FNAL in the fall in 2003 (Figure 5.6). Three stations were constructed for the prototype. Two of three stations are made with two views and each view has different concentration of $3 \mathrm{HF}$. The specification of the prototype tracker is described in Table 5.4. 


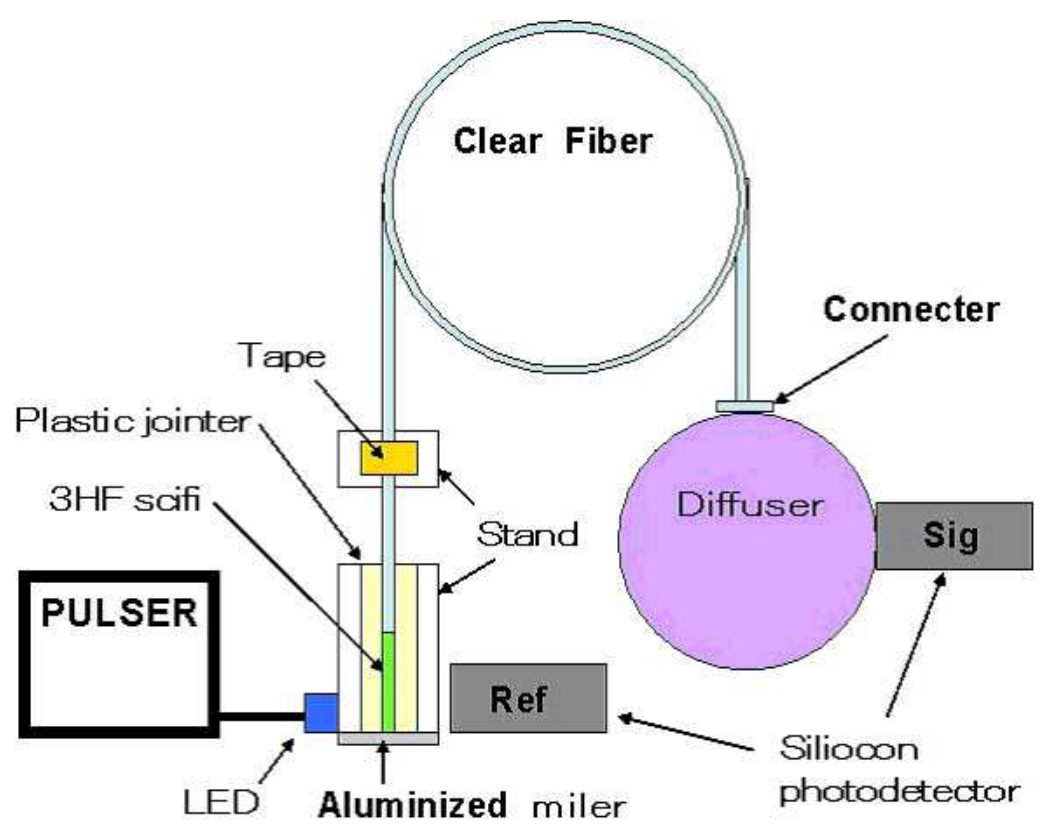

Figure 5.3: Schematic view of set up in the attenuation measurement of clear fiber.

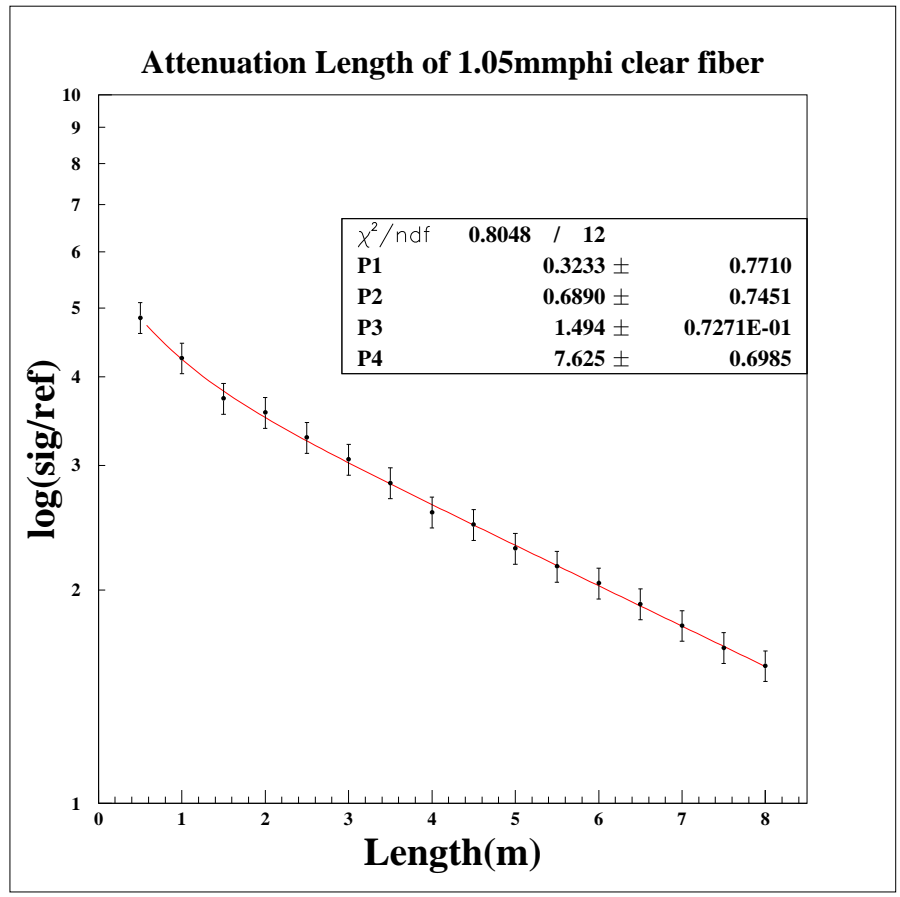

Figure 5.4: The result of attenuation measurement in clear fiber. Horizontal axis shows the length of clear fiber, and vertical is the measured luminosity after transmission in clear fiber with respect to the light yield of the LED source. 

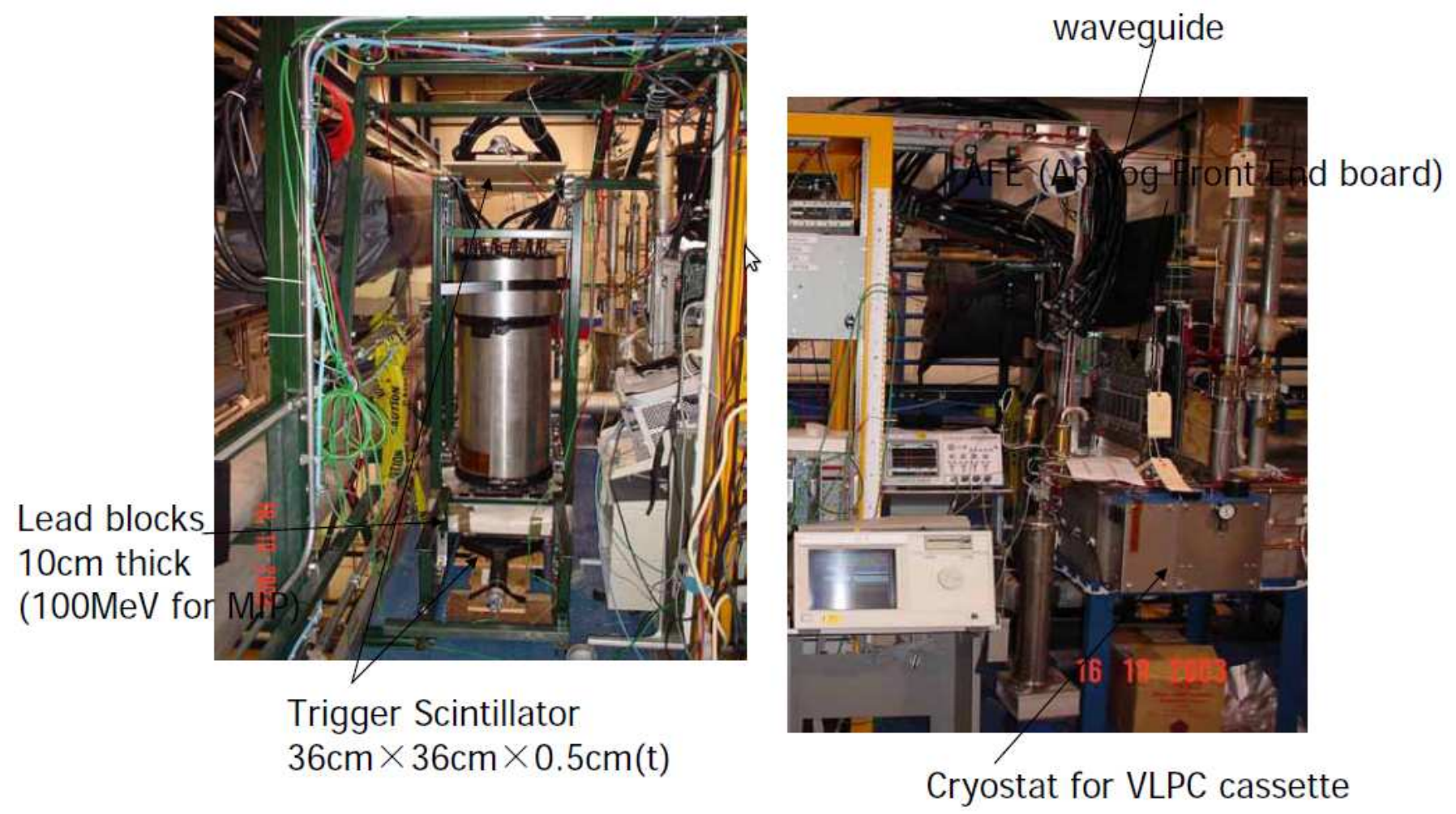

Figure 5.5: Photograph of the cosmic-ray test stand at D0 for prototype tracker (left). Trigger counters are located upside and downside of the tracker. Light-guides extracted from light-tight storage tube are attached with the optical connectors mounted on the cyrostat (right).

\begin{tabular}{ccc}
\hline Station & View & 3HF concentration $(\mathrm{ppm})$ \\
\hline $\mathrm{A}$ & $\mathrm{X}$ & 5000 \\
$\mathrm{~A}$ & $\mathrm{~V}$ & 5000 \\
\hline $\mathrm{B}$ & $\mathrm{X}$ & 5000 \\
$\mathrm{~B}$ & $\mathrm{~V}$ & 2500 \\
$\mathrm{~B}$ & $\mathrm{~W}$ & 3500 \\
\hline $\mathrm{C}$ & $\mathrm{X}$ & mixture \\
$\mathrm{C}$ & $\mathrm{W}$ & 5000 \\
\hline
\end{tabular}

Table 5.4: List of 3HF concentration at each views in the prototype tracker. 


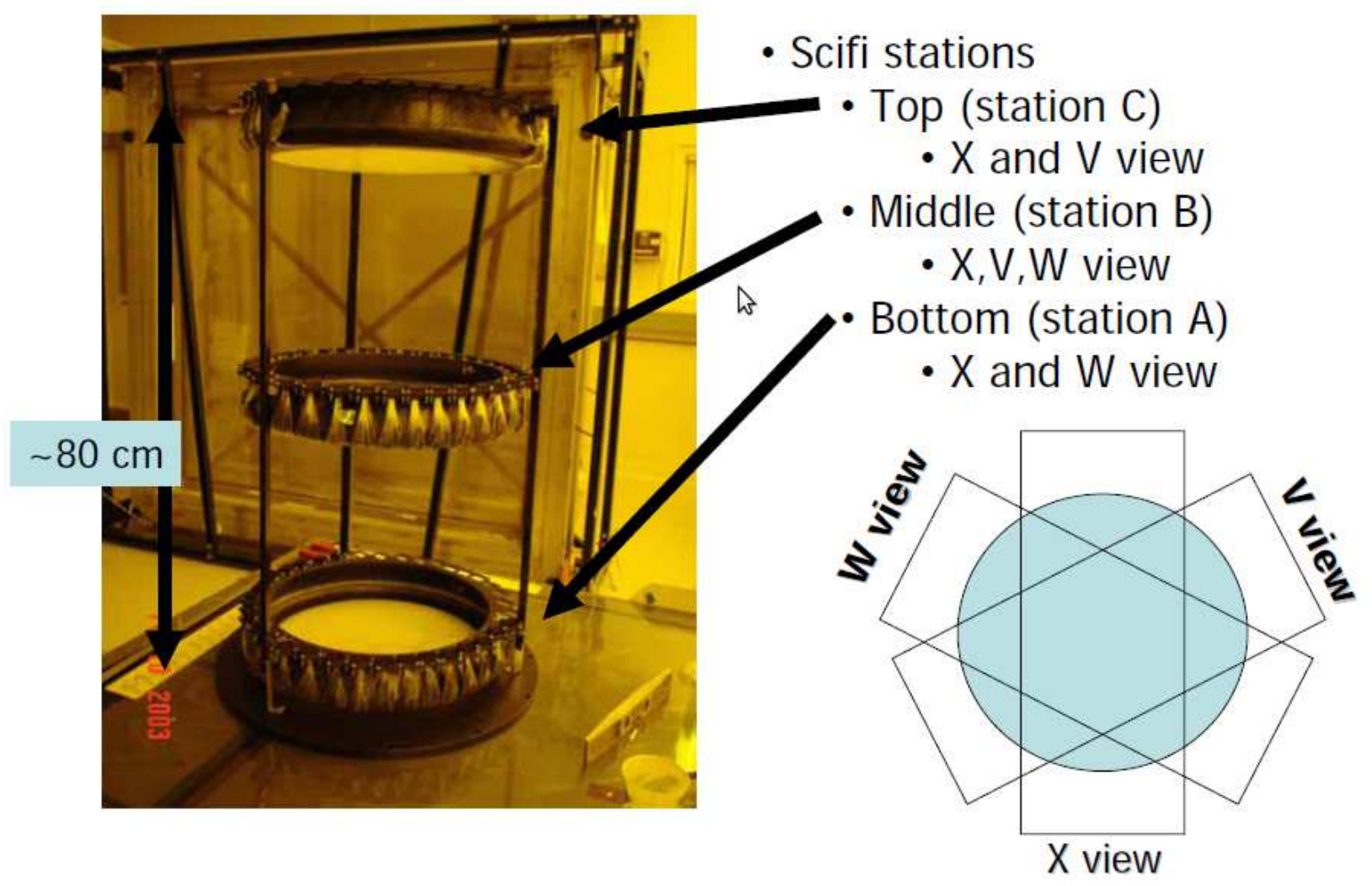

Figure 5.6: Photograph showing the three stations constructed for the cosmic-ray test.

126 channel / waveguide
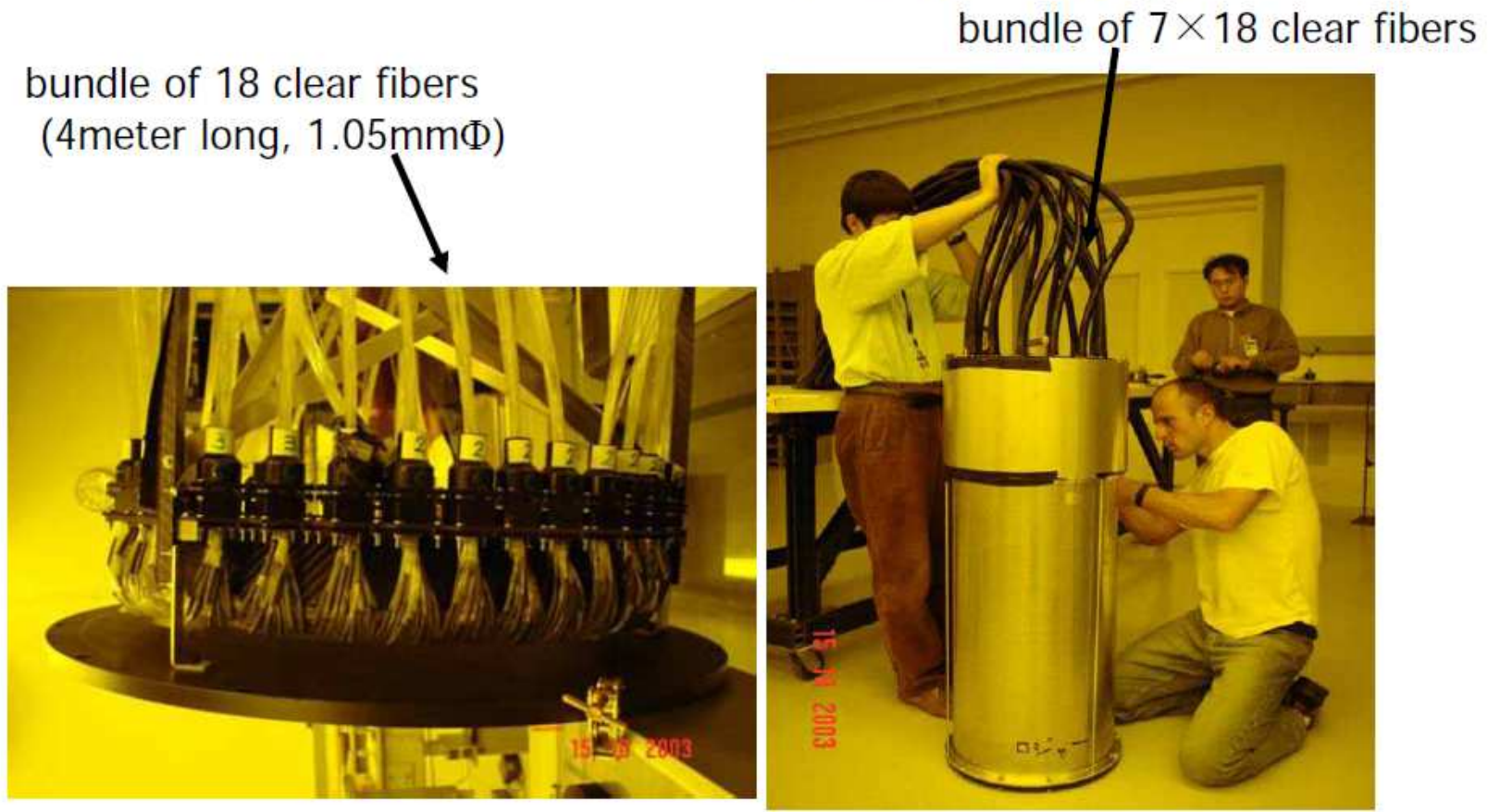

Figure 5.7: External light-guides are directly attached with the station connectors (left) and extracted from the path panel (right). 


\subsubsection{Light-guide}

Light-guides of $4 \mathrm{~m}$-long external light-guides are attached with stations and extracted from the small patch panel from top side (Figure 5.7).

\subsubsection{VLPC cryostat}

VLPC cryostat used for the cosmic-ray test was needed to supply and return Helium gas externally, which was used in the D0 test stand. MICE have been made new standalone cryostats with cryocooler to save costs for the infrastructure of external Helium gas flow system. The new designed cryostat (prototype) have been tested in the KEK beam test in 2007.

\subsubsection{Trigger counter}

Two sets of plastic scintillating counters are located at top and bottom of the prototype tracker, each are separated by about $2 \mathrm{~m}$. The dimension of a scintillator is $36 \mathrm{~cm} \mathrm{x} 36 \mathrm{~cm} \mathrm{x} 0.5 \mathrm{~cm}$ (thickness). On both sides of the scintillator, fish-tale light-guide are attached. A 2-inch PMT is glued with light-guide. The lead block with $10 \mathrm{~cm}$ thickness is put on the bottom counter.

\subsubsection{Read-out}

\subsubsection{Trigger logic}

The discriminated signals from four PMTs and the output signal generated by a pulse generator DG2020 are 'AND'ed at a coincidence module. The signal from the coincidence module is then directed to the Avenet board, where trigger signal is sent to the AFEII board via a flat cable.

The trigger rate made by two trigger counters is $0.5 \mathrm{~Hz}$ and the rate accepted to AFEII board (L1Accept) is $0.04 \mathrm{~Hz}$.

\subsubsection{Electronics}

\section{AFEII board}

Four AFEII boards are used for the test. The total number of channels is $2048(512 \times 4)$.

\section{VLSB board}

Four VLSB boards are slotted in the VME crate and connected with the AFEIIt backplane via LVDS cables.

\subsubsection{DAQ and slow control}

Both DAQ and slow control are operated by Excel VBA (Visual Basic for Application) developed by the D0 group. The AFEII boards are needed to be initialized before starting data-taking. 


\subsubsection{Light-yield measured with different kinds of concentrations of $3 \mathrm{HF}$}

The light yield is estimated by dividing pedestal-subtracted ADC counts by a gain. The gain is defined as ADC counts per one photo-electron, which is measured by another LED run. The typical ADC distribution measured at a LED run is shown in Figure 4.6.

The light yield on $\mathrm{X}$ view at the middle station $\mathrm{B}$ is estimated as follows. A linear track is made using hits on outer stations, $\mathrm{A}$ and $\mathrm{C}$. The track is interpolated to the middle station $\mathrm{B}$ and residuals to hit fibers on station $B$ are calculated. The fiber of the smallest residual is chosen and its light-yield is calculated. The measured light yield on three views at station B are shown in Figure 5.8.

The result shows that the combination of scintillating fiber and clear fiber selected are good enough for used at MICE SciFi tracker.

The light-yield distribution measured for each concentration are shown in Figure 5.8 and their fitted means are summarized in Table 5.5. From this result, the concentration of 3HF is determined to be $2500 \mathrm{ppm}$.

\begin{tabular}{ccc}
\hline Station & 3HF concentration (ppm) & Light yield (p.e.) \\
\hline B & 5000 & $10.6 \pm 0.3$ \\
B & 3500 & $13.4 \pm 0.4$ \\
B & 2500 & $13.6 \pm 0.5$ \\
\hline
\end{tabular}

Table 5.5: Light-yields measured at the prototype tracker.
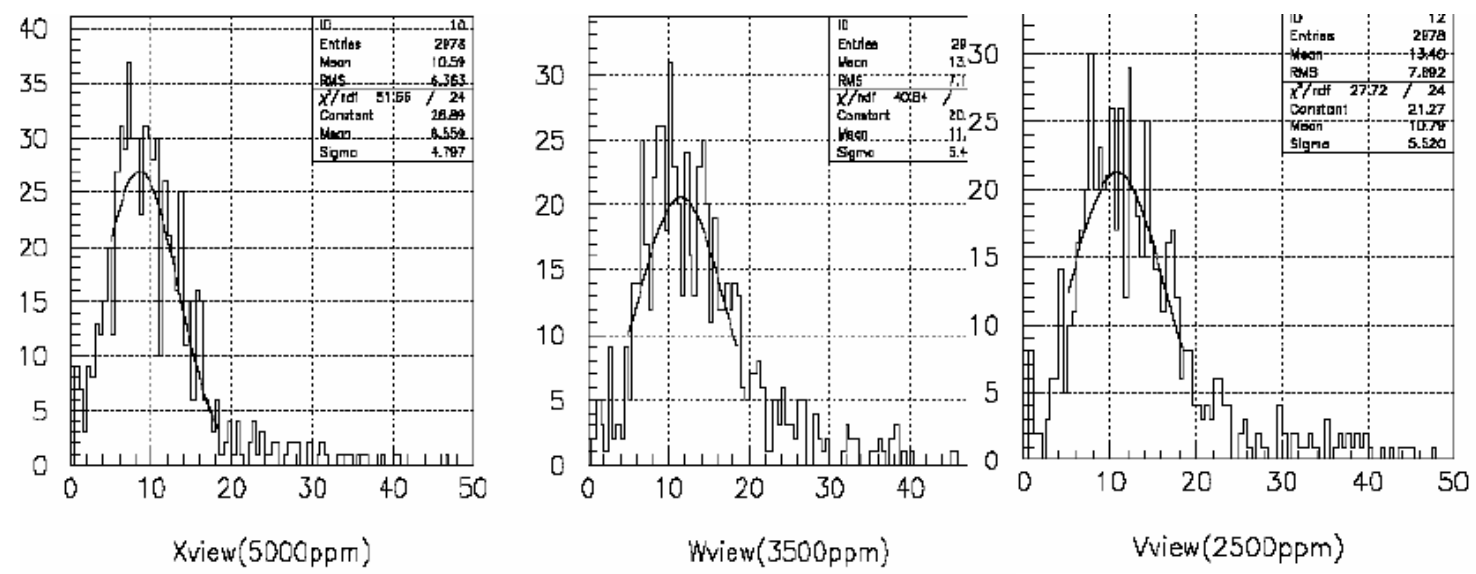

Figure 5.8: Light-yield distribution measured at three different kinds of 3HF concentration at the cosmic-ray test.

\subsection{Beam test at KEK}

The prototype tracker have been tested under 1T magnetic fields at the KEK $\pi 2$ beam line in 2005 . Fourth station has been additionally installed in the prototype tracker tested with cosmic-ray test in the FNAL. Transverse momentum are made by rotating the spectrometer by 3 or 6 degrees 
with respect to the beam line. Using muons of energy $250 \mathrm{MeV} / c, 350 \mathrm{MeV} / c$ and $425 \mathrm{MeV} / c$, transverse momentums are measured by reconstructing the tracks.

The setup of the beam test are described at first, then the reconstruction method applied and the reconstructed momentum distribution are shown.

\subsubsection{Setup}

Set up of counters and tracker installed in a spectrometer magnet is shown in Figure 5.9;

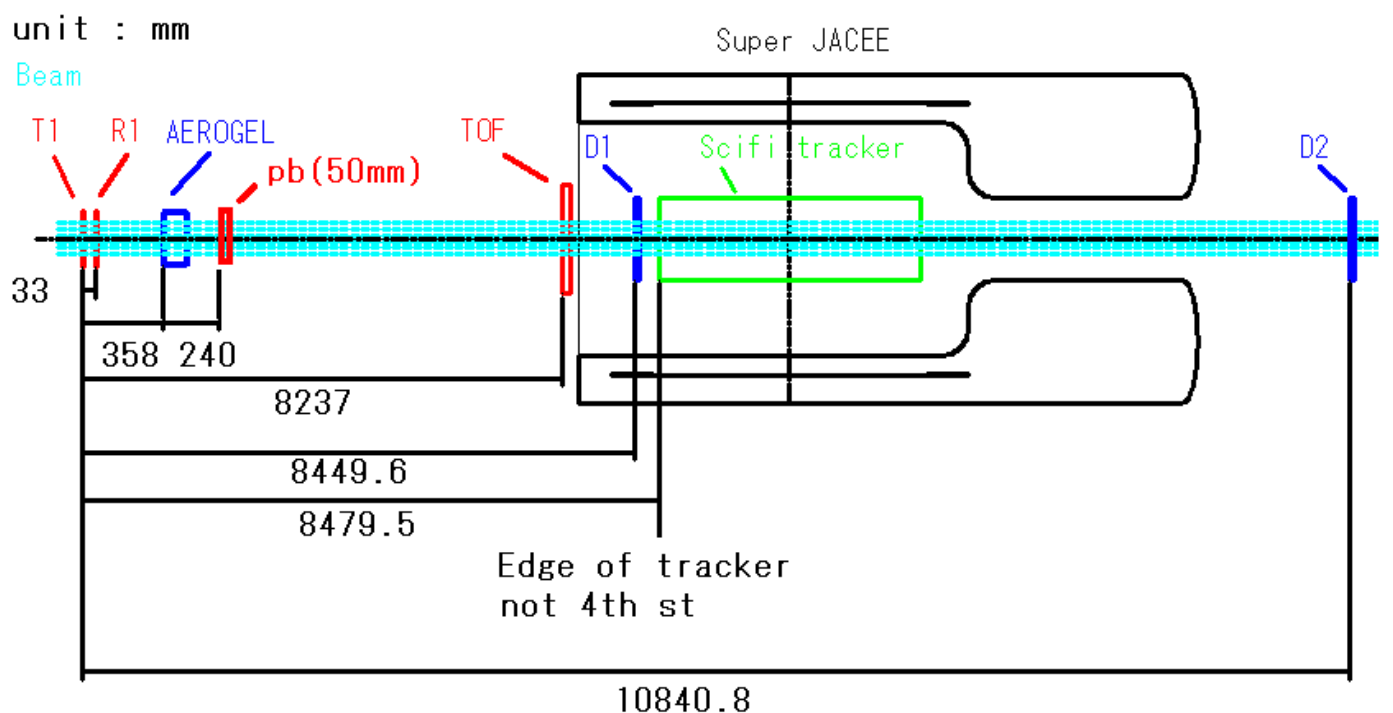

Figure 5.9: Setup of KEK beam test

\subsubsection{Trigger counter}

A scintillating counter of $5 \mathrm{~cm}$ height, $10 \mathrm{~cm}$ wide and $2 \mathrm{~cm}$ thickness is located at the most upstream of the experimental area. The scintillating-light is read out by two PMTs attach at both ends of scintillator.

\subsubsection{Cherenvkov counter}

The cherenkov counter made of seven layers of aerogel block $(10 \mathrm{~cm} x 10 \mathrm{~cm} 1.1 \mathrm{~cm}$ thickness) is installed to identify electrons and muons at low momentum. A refractive index of aerogel is 1.05. The aerogel block is mounted in the light-tight box at which inside wall a light reflective material is coated. Ten PMTs are attached with the box for read-out.

\subsubsection{TOF hodoscope}

A TOF hodoscope is composed of ten slabs of scintillator bars of $40 \mathrm{~cm}$ long, $8 \mathrm{~cm}$ wide and $2 \mathrm{~cm}$ thickness (Figure 5.10). Five slabs are horizontally aligned and another five are vertically aligned. Each slab is read out by two PMTs, HAMAMATSU R6504S, fine mesh, 2.5 inch, which 


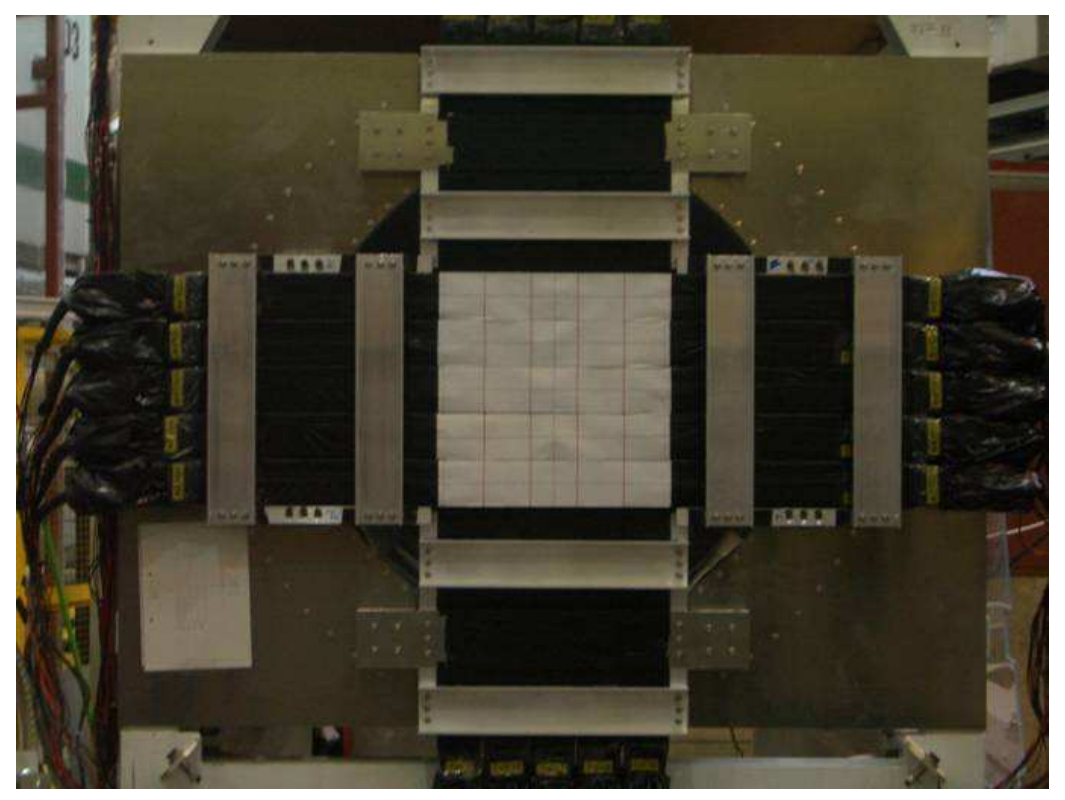

Figure 5.10: Photograph of TOF hodoscope used for the beam test viewed from the upstream.

has tolerance to the magnetic field. A PMT is glued with a light-guides of $52.5 \mathrm{~cm}$ long. Two sets of light-guides are glued with the scintillating bar. The light-guide is designed so that light-yield loss in light-guide is as much as small to keep the time resolution to $55 \mathrm{ps}$ and a direction of stray field becomes parallel to the PMT to keep the PMT gain. The magnitude of the magnetic field at a PMT is estimated to 1.1 KGauss.

\subsubsection{Beam defining counter}

Two sets of scintillating counters of $30 \mathrm{~cm}$ diameter with $0.5 \mathrm{~mm}$ thickness are located at the upstream and downstream of the tracker. A beam defining counter is read out by a PMT using clear-fiber light-guide of about $3 \mathrm{~m}$ long and fixed with the support frame of the magnet, where the magnitude of the magnetic field is so small that a PMT can maintain its gain.

\subsubsection{Spectrometer}

\subsubsection{Superconducting magnet}

The superconducting magnet which produces $1 \mathrm{~T}$ uniform magnetic field is used. The diameter of the bore is $85 \mathrm{~cm}$. The prototype is mounted inside of the magnet. On the front of the magnet an aluminium plate with the TOF hodoscope mounted is attached. The magnet is sit on the support stand to adjust the height so that the beam passes through the center of the prototype tracker. Wheels are attached at the bottom of the support stand to change the orientation of the magnet easily.

\subsubsection{Prototype tracker}

The prototype tracker tested is composed of four stations, three of these are stations used at the cosmic-ray test at the FNAL. The design of fourth station is almost the same as other three except 


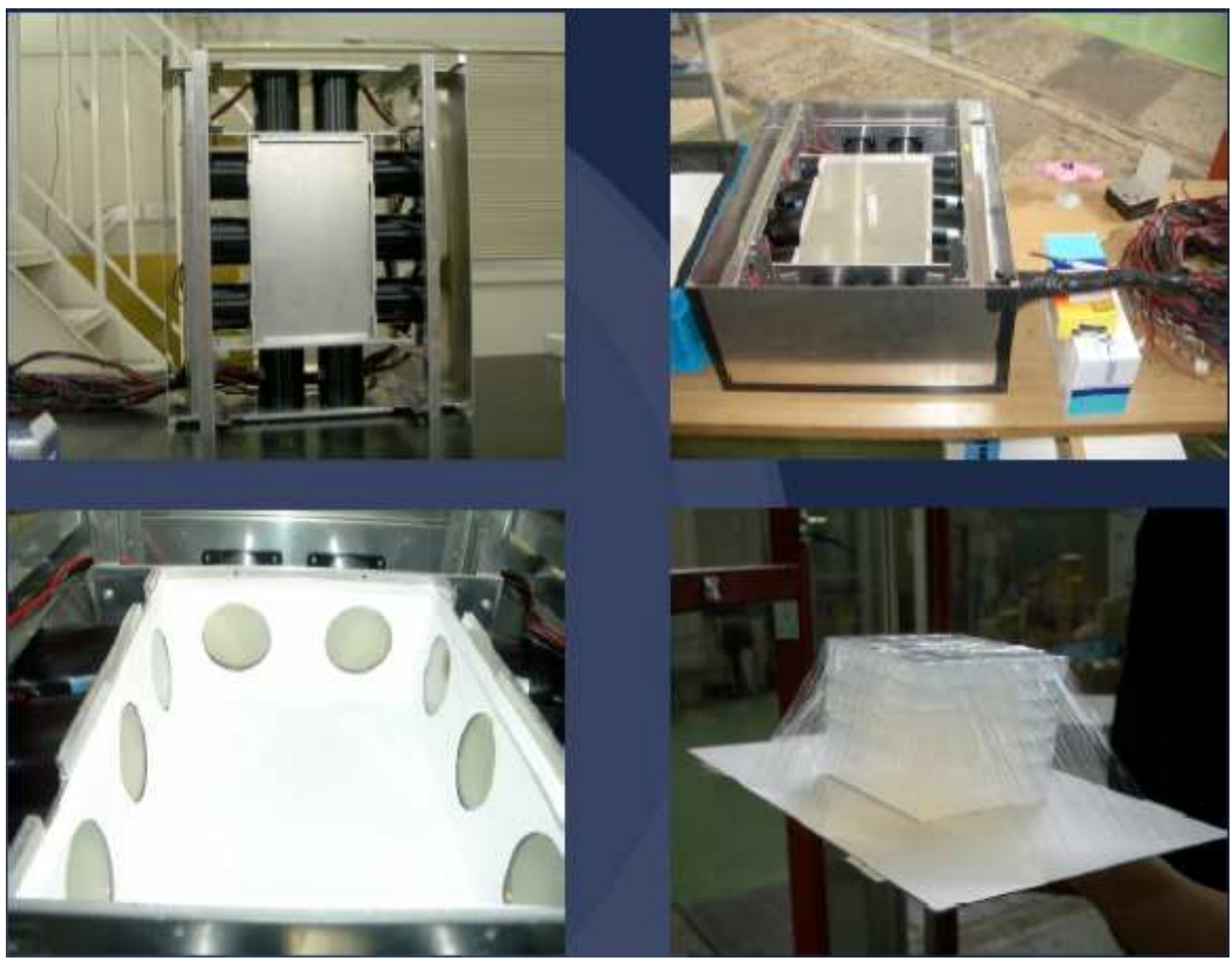

Figure 5.11: Photograph showing aerogel counter used at the beam test.

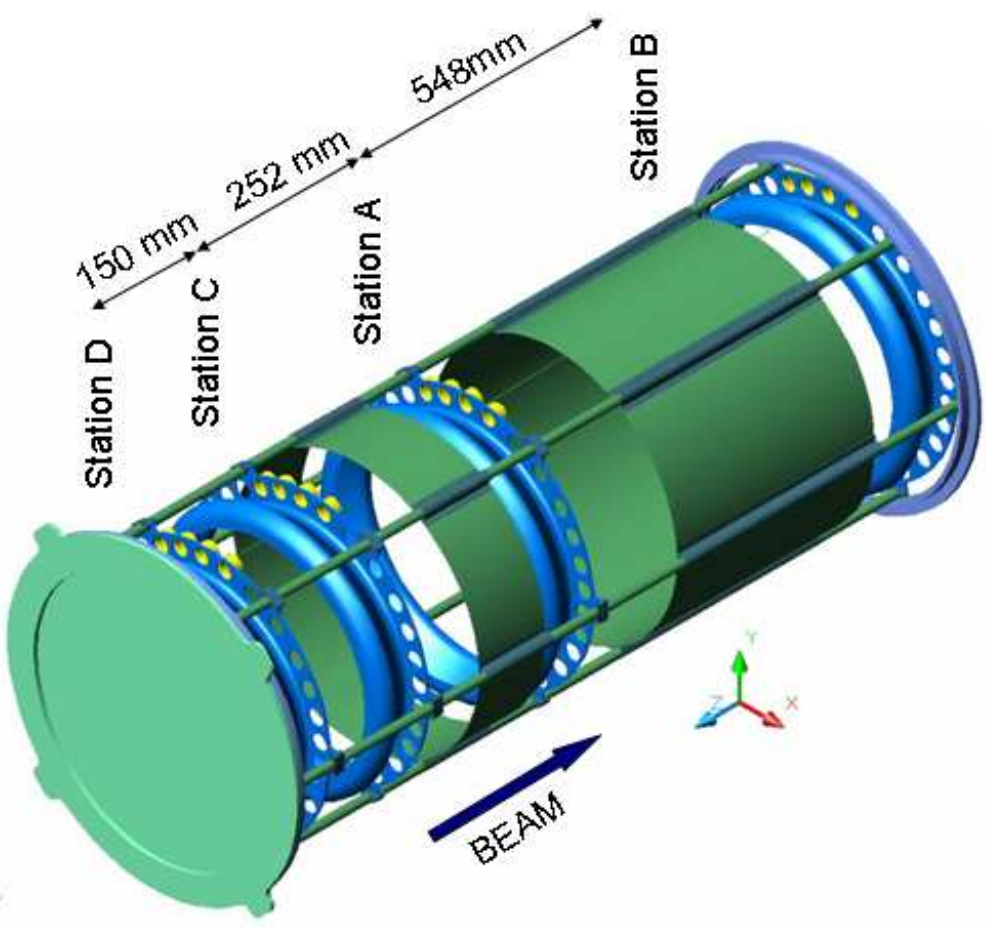

Figure 5.12: Schematic view of the prototype tracker of four stations without light-guides attached. 
for the optical connector. This newly designed connector is threaded around the connector so that connectors can be fixed easily by turning itself, while original one is needed for screws.
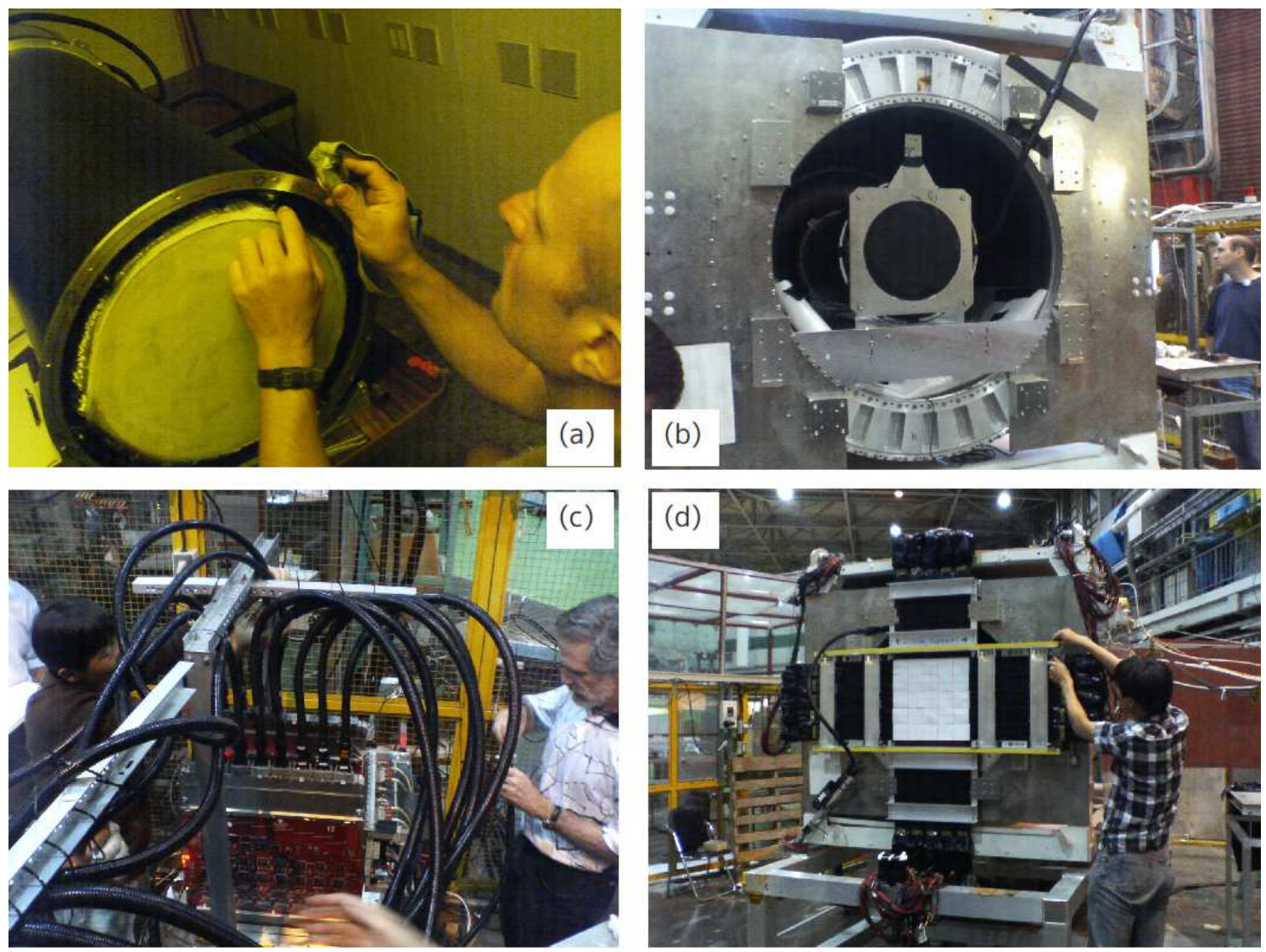

Figure 5.13: Photographs showing the construction of the prototype tracker into the light-tight tube (a), installed into the spectrometer magnet (b), light-guides are being attached on the modules on the cryostat (c), and the TOF hodoscope is attached at the front of the spectrometer (d).

\subsubsection{VLPC cassette with cryocooler}

New designed and developed cassette with cryocooler for MICE have been tested in the beam test.

\subsubsection{Read-out}

\subsubsection{Trigger logic}

The trigger (L1Accept) is generated by the coincidence of a beam trigger and a AFEII clock signal. The beam trigger is generated when the particle passes through T1, any of vertical slabs of the hodoscope, any of horizontal slabs of the hodoscope and D1 counter. The AFEII clock signal is generated using DG2020 pulse generator and sent to the AFEII board. When the timing of the bream trigger matches the AFEII clock, the L1Accept is generated. The L1Accept signal is then 
transfered to the AFEII board using the Avnet board to perform the digitization and sent data to the VLSB board.

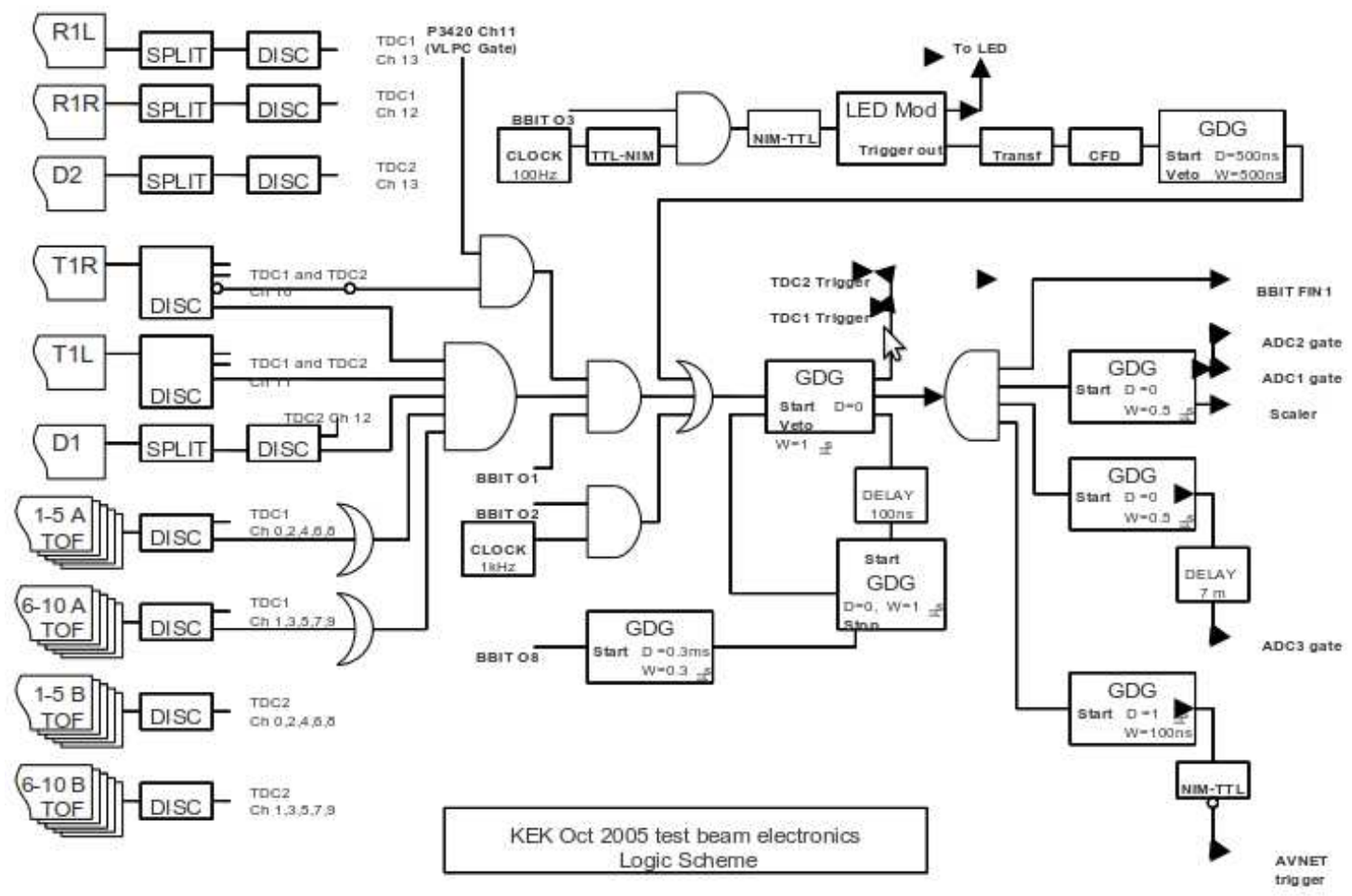

Figure 5.14: Trigger logic used for the beam test.

\subsubsection{Electronics for tracker}

\section{AFEII board}

Four AFEII boards are used. Each board has 512 channels. Data is transferred to VLSB modules via LVDS cables.

\section{VLSB board}

Four VLSB boards are used, each are connected with the AFE backplane via four LVDS cables.

\section{DG2020 pulse generator}

The pulse generator is used for sending external trigger into AFEII boards.

\section{Avnet board}

The Avnet board is used to sending external trigger to AFEII board from the trigger counters. 


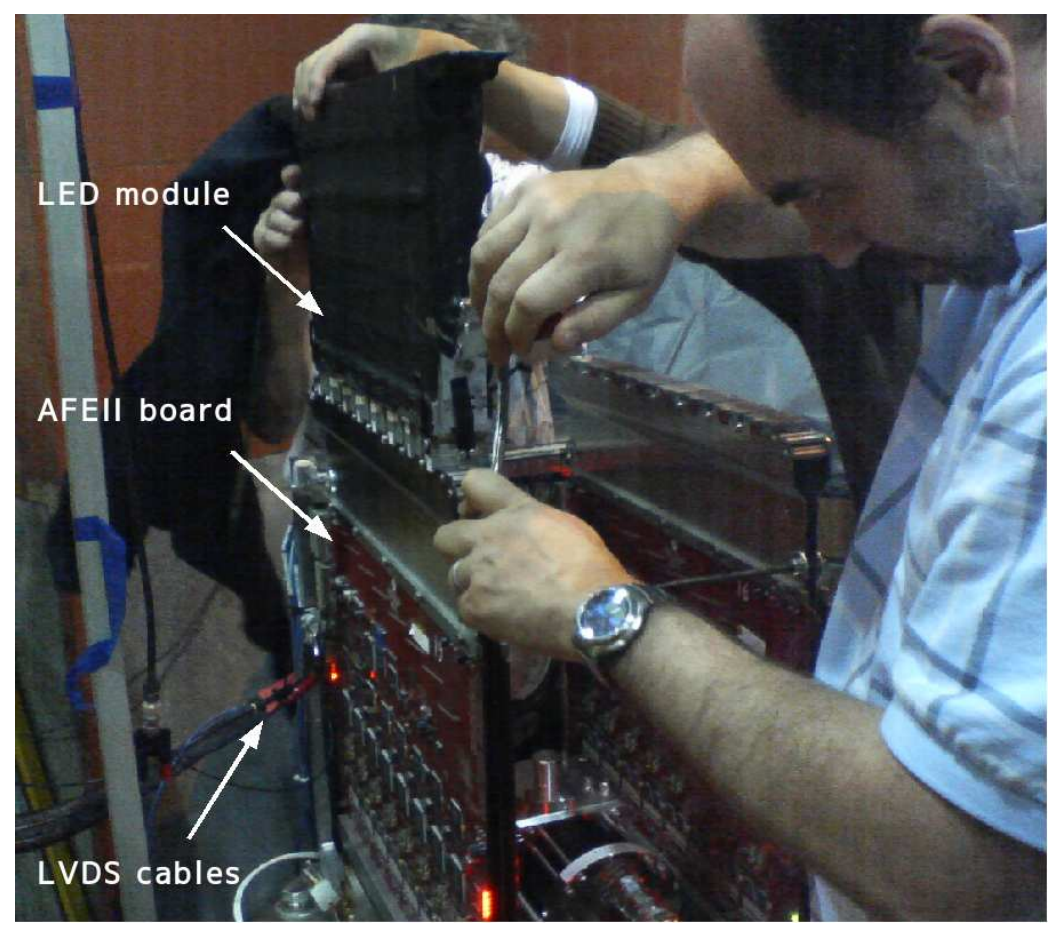

Figure 5.15: Photograph of AFEII baords used at the beam test. Four boards are connected in the AFEII backplane on the cryostat. LVDS cables are attached to connect VLSB boards in VME crate.

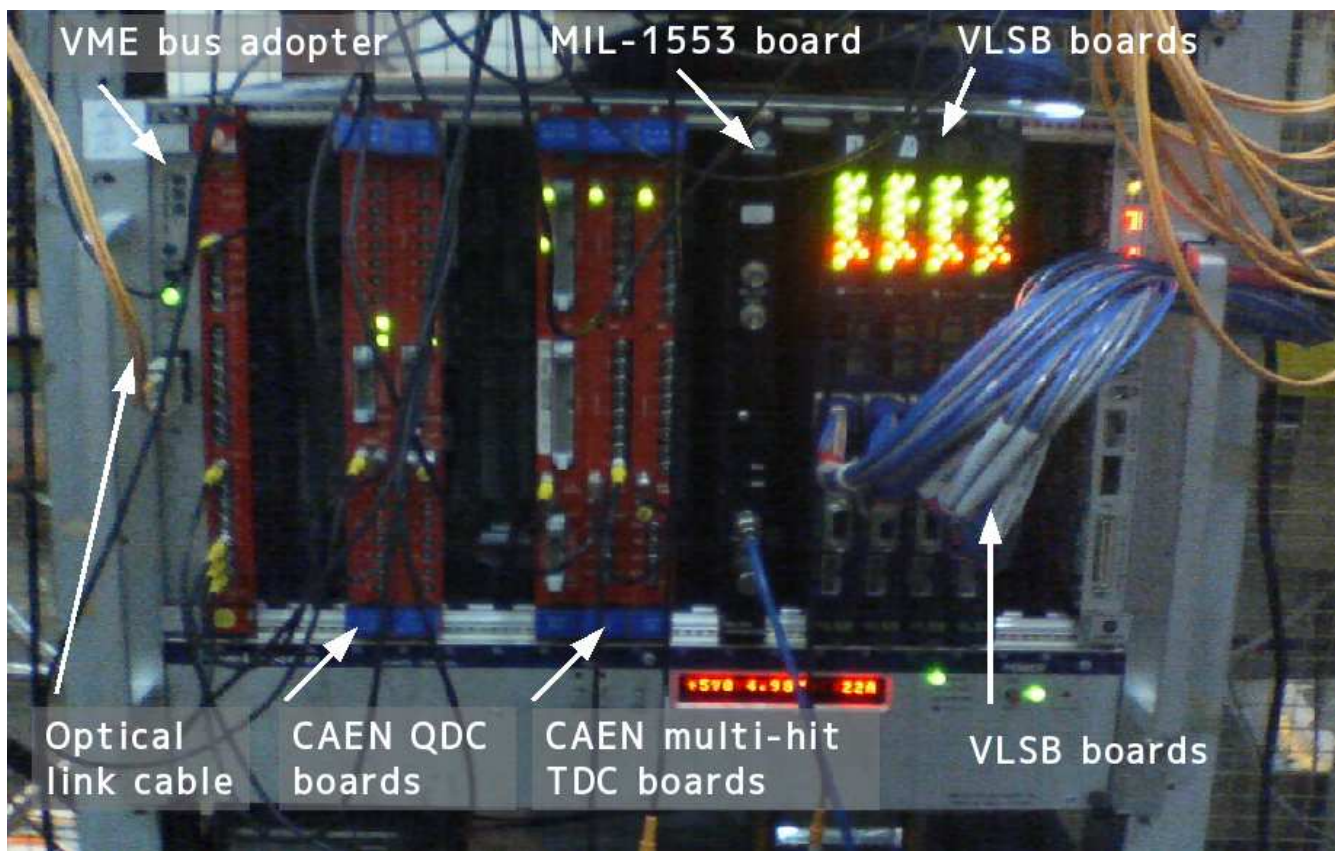

Figure 5.16: Photograph of the VME crate and VME modules used at the beam test. 


\subsubsection{Electronics for beam monitors}

\section{CAEN V792}

The CAEN V792N is the charge-sensitive ADC module used to measure pulse height of PMT signals.

\section{CAEN V1290}

The CAEN V1290N is the multihit TDC module of 25 ps resolution used to measure hit timing.

\subsubsection{DAQ and slow control}

The DAQ is implemented based on the UNIDAQ framework, developed by KEK-online group. In the UNIDAQ, several processes are running in parallel on single machine or multiple machines to accomplish the task. The NOVA process has buffer and manages data which is transfered among processes. The collector process read out data stored in CAMAC/VME modules. The receiver process transfers data to another host if requested. The eventBuilder process merges data which is recorded in several CAMAC/VME boards to make single event structure. The recorder process records data in the local disk.

In the test, collectors for VLSB board, CAEN V1290 and CAEN V792 are implemented. The read-out is carried out at every time when the trigger (L1Accept) reaches to the DAQ system.

The slow control has responsibility to initialize AFEII baords before stating data-taking. This is done by the EXCEL VBA program on the Windows PC.

\subsubsection{Track reconstruction}

In order to calculate the transverse emittance, hit positions and transverse and longitudinal momentums are needed to be solved for individual muons. Hit positions are directly solved by hit fiber numbers (digitized by the pitch of fibers). Transverse $\left(p_{T}\right)$ and longitudinal $\left(p_{L}\right)$ momentums are resolved by reconstructing helical trajectories with software from the hit positions.

Some techniques are proposed for the reconstruction. G4MICE (software tools developed for MICE) uses Kalman filter algorithm, which considers the effects of multiple scattering and errors of magnetic fields. In the analysis of the KEK beam test, most simplest algorithm, which reconstructs muon tracks as helical tracks which is the true in the case no scatterings are observed. This method will be referred to as 'helical tracking' in the following. Using this method, muons taken at the beam test are reconstructed. In chapter 8 , the helical tracking will be used to evaluate the systematic error on emittance measurement.

Next subsections describes on this method briefly and the observed momentum distributions.

\subsubsection{Helical tracking}

Helical motion in the uniform magnetic field along the z-axis can be formed using five parameters as shown in Figure 5.17). Best values of parameters are determined so as to minimize the 'chi-squared" function. 


\section{Helical Tracking}

$$
\begin{gathered}
\chi^{2}=\sum_{\mathrm{i}} \frac{(\mathrm{xi}-\mathrm{xfit})^{2}}{\sigma^{2}}+\frac{(\mathrm{yi}-\mathrm{yfit})^{2}}{\sigma^{2}} \\
\mathrm{x}_{\mathrm{fit}}=\mathrm{x}_{0}+\mathrm{R} \cos \left[\mathrm{z}_{\mathrm{i}} / \mathrm{L}+\phi_{0}\right] \\
\mathrm{y}_{\mathrm{fit}}=\mathrm{y}_{0}+\mathrm{R} \sin \left[\mathrm{z}_{\mathrm{i}} / \mathrm{L}+\phi_{0}\right] \\
\mathrm{R}=\mathrm{p}_{\mathrm{T}} /(0.3 \mathrm{~B}) \\
\mathrm{L}=\mathrm{p}_{\mathrm{L}} /(0.3 \mathrm{~B})
\end{gathered}
$$

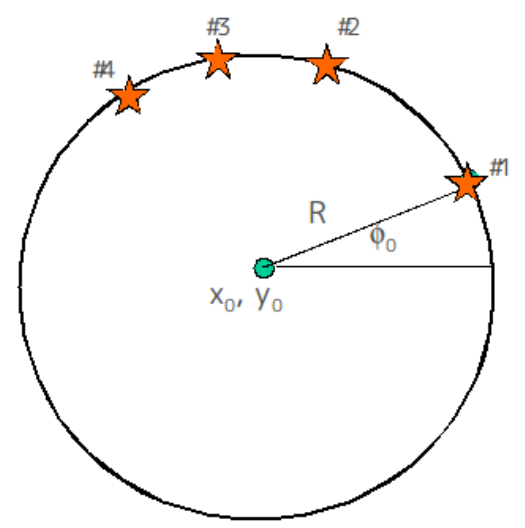

Figure 5.17: Definition of helical fitting parameters and chi-squared function to be minimized. $x_{i}$, $y_{i}$ are measured $\mathrm{x}, \mathrm{y}$ positions and $x_{f i t}, y_{f i t}$ are expected positions which are given by five fitting parameters, $x_{0}, y_{0}, \mathrm{R}, \mathrm{L}$, and $p_{0}$. Transverse $\left(p_{T}\right)$ and longitudinal $\left(p_{L}\right)$ momentum are calculated by $\mathrm{R}$ and $\mathrm{L}$, respectively.

\subsubsection{Reconstructed momentum distribution}

Reconstructed transverse and longitudinal momentums are shown in Figure 5.18 and Figure5.19, respectively. With respect to the transverse momentum, momentum shifts corresponds to magnet angles are apparently seen. For the longitudinal momentum, there are agreements with the time of flight measurement by about $8 \%$. 


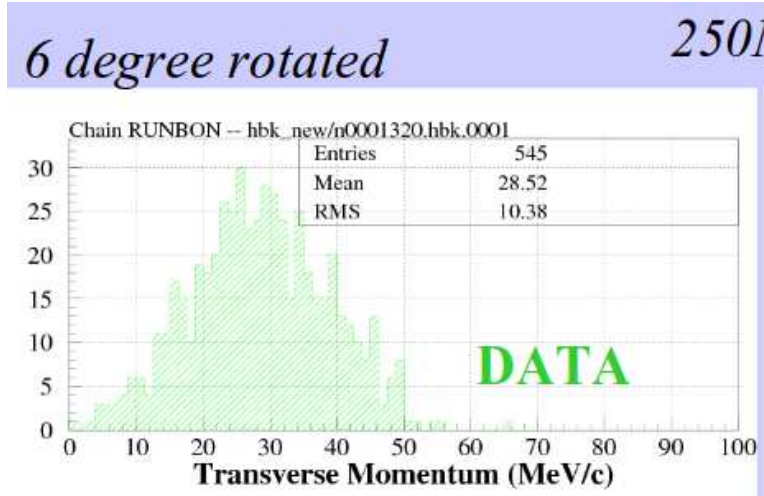

$250 \mathrm{MeV} / \mathrm{c}$

3 degree rotated
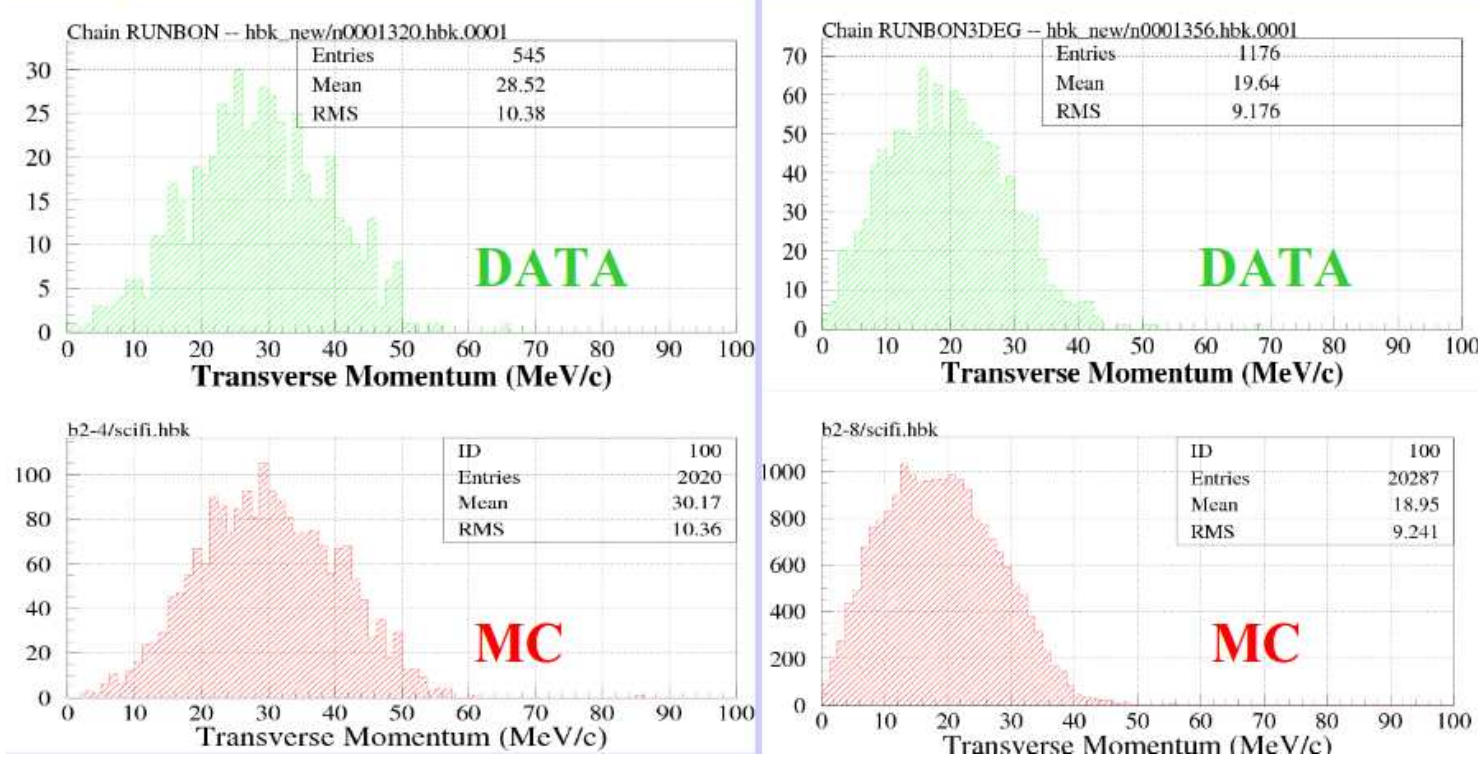

Figure 5.18: Reconstructed transverse momentum distributions. Green histograms on top are results of data and red histograms at bottom are expectation by a Monte-Carlo simulation. Left (right) corresponds to the result with the magnet rotated by 6 (3) degrees.
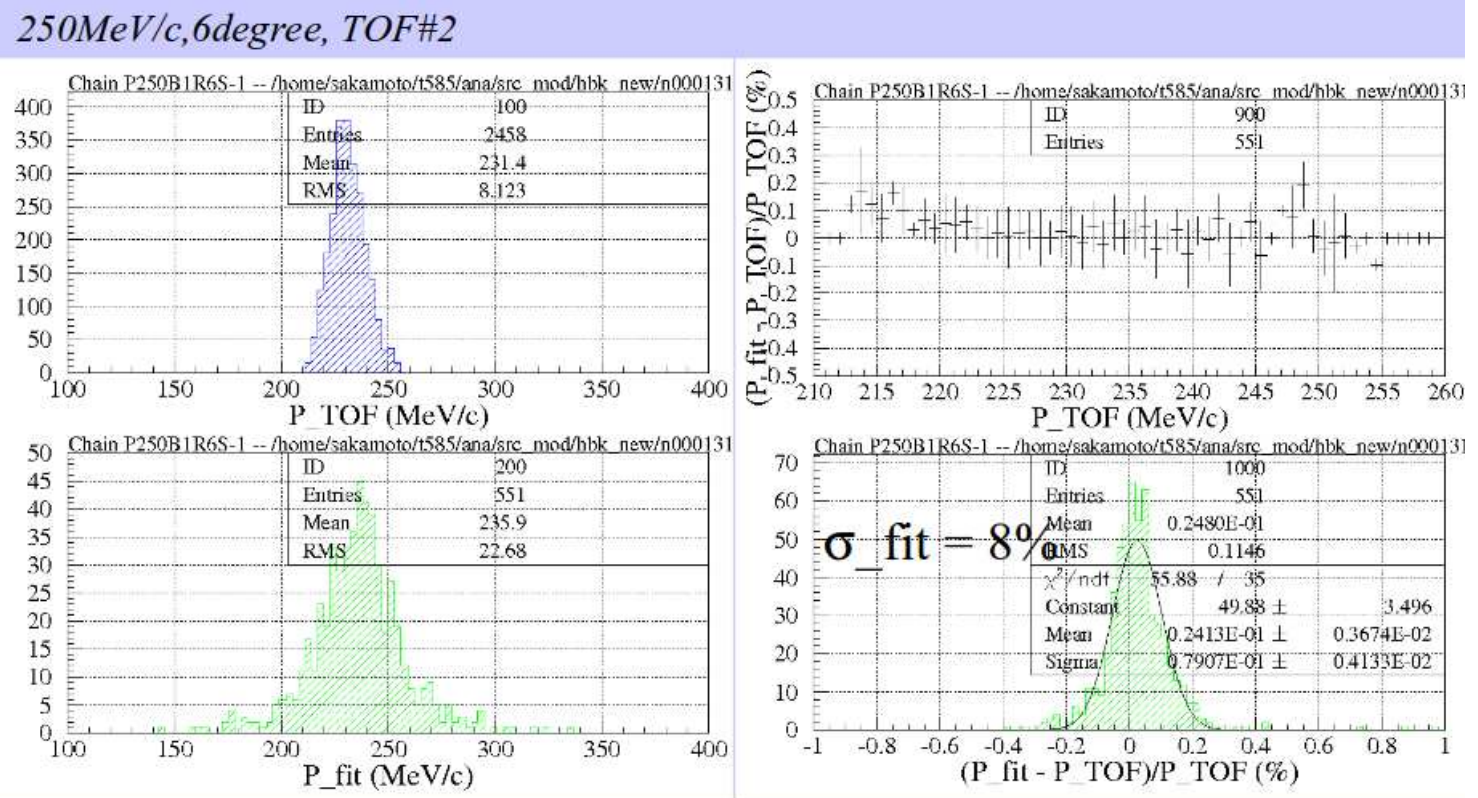

Figure 5.19: Reconstructed longitudinal momentum distributions and residual to momentum measured with the TOF measurement. 


\section{Chapter 6}

\section{SciFi Tracker Production}

\subsection{SciFi stations}

Fifteen SciFi stations including spares have been produced with the quality assurance (QA) procedures applied. At the light-yield measurement using a ${ }^{57} \mathrm{Co}$ gamma-ray source, light yields of more than 10 photo-electrons have been measured for all the view of the stations. The following sections describe the processes to produce SciFi stations and also the quality assurance procedures adopted for the station production.

\subsubsection{Fabrication}

The SciFi doublet ribbons were built at the Fermi National Accelerator Laboratory (FNAL) in the USA. The SciFi stations were fabricated from the SciFi doublet ribbons at Imperial College (IC) London, UK in years of 2008 and 2009. Figure 6.2 shows a picture of the station No.5, which was firstly constructed at the IC. In following, each procedure of the fabrication held at the IC is described.

\subsubsection{Bundling/Connecting}

Groups of adjacent seven scintillating fibers were bundled together from a center fiber of a SciFi doublet. A bundle was threaded through a small rubber tube (Figure 6.3 (a)). After bundling, it was checked that seven scifis were bundled into rubber tubes in sequence without skipping. This 'bundling-and-error-check' was carried out every after twenty bundles or so were made to minimize possible corrections. The detail on this test is described in the subsection 6.2.3. Once all the bundles were made and tested, they were attached with 22-way optical (station) connectors. As described in section xxx, ten connectors were attached per doublet (view), in two of which has only 20 channels are used ( 2 channels are empty). Bundles were threaded into holes on the station connectors. After that, errors on threading into wrong holes were examined (see Section 6.2.3).

\subsubsection{Gluing/Polishing}

The doublet with the station connectors was then located on the vacuum chuck in such a way that a center fiber (colored with black) was aligned to the middle of the vacuum chuck. The alignment was checked by a microscope mounted above the vacuum chuck, then fixed on the vacuum chuck by evacuating air between them. The station body with glue applied on its circumference was put on 


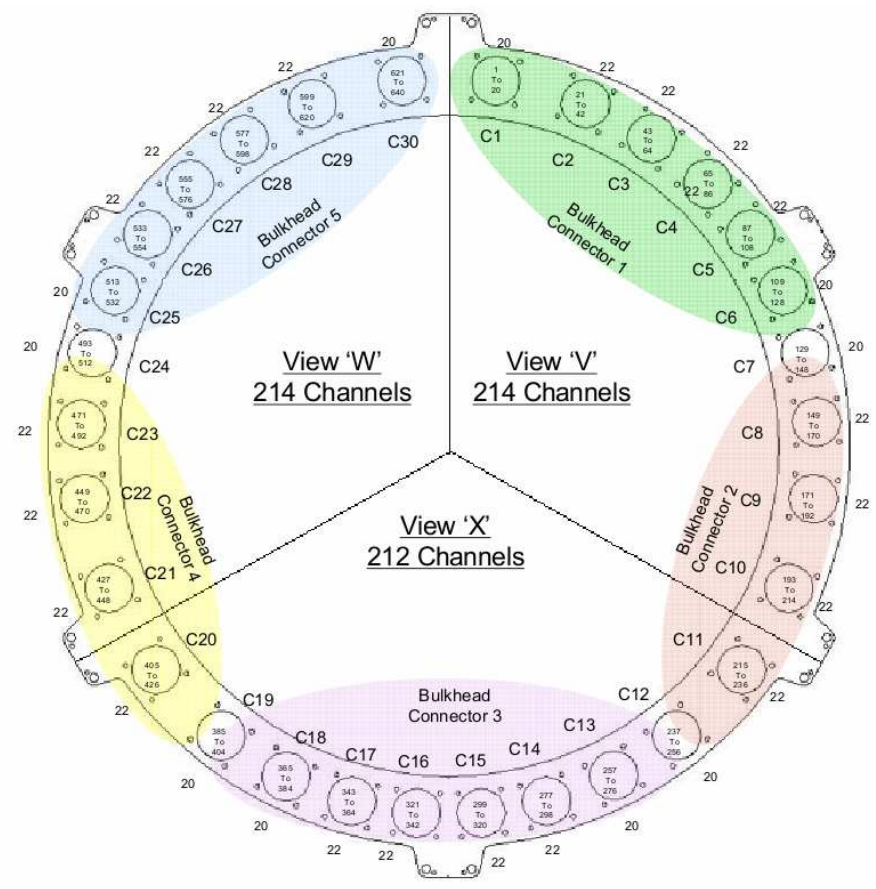

Figure 6.1: Schematic view of station. Scintillating fibers are aligned in three views (ribbons) V, $\mathrm{X}$ and $\mathrm{W}$ view, each of them are located every 120 degrees. There are thirteen optical (station) connectors around the station frame where internal light-guides are attached.

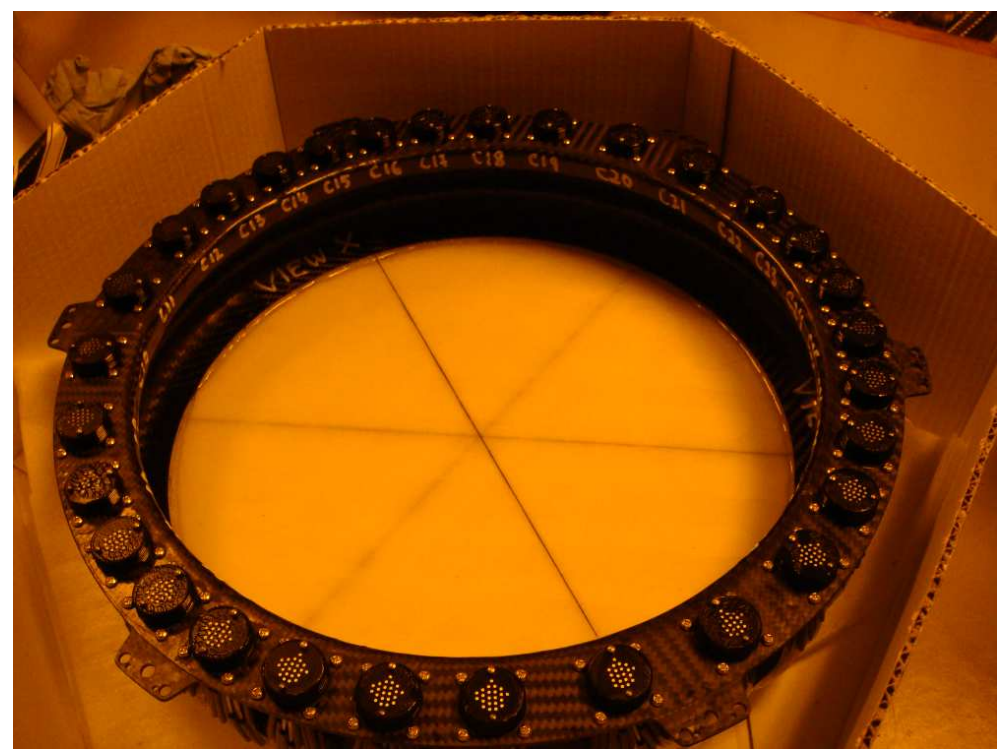

Figure 6.2: Station No.5 for Tracker No.1 firstly produced at Imperial College London. Three back lines seen in the double layer corresponds to center fibers in each views. 

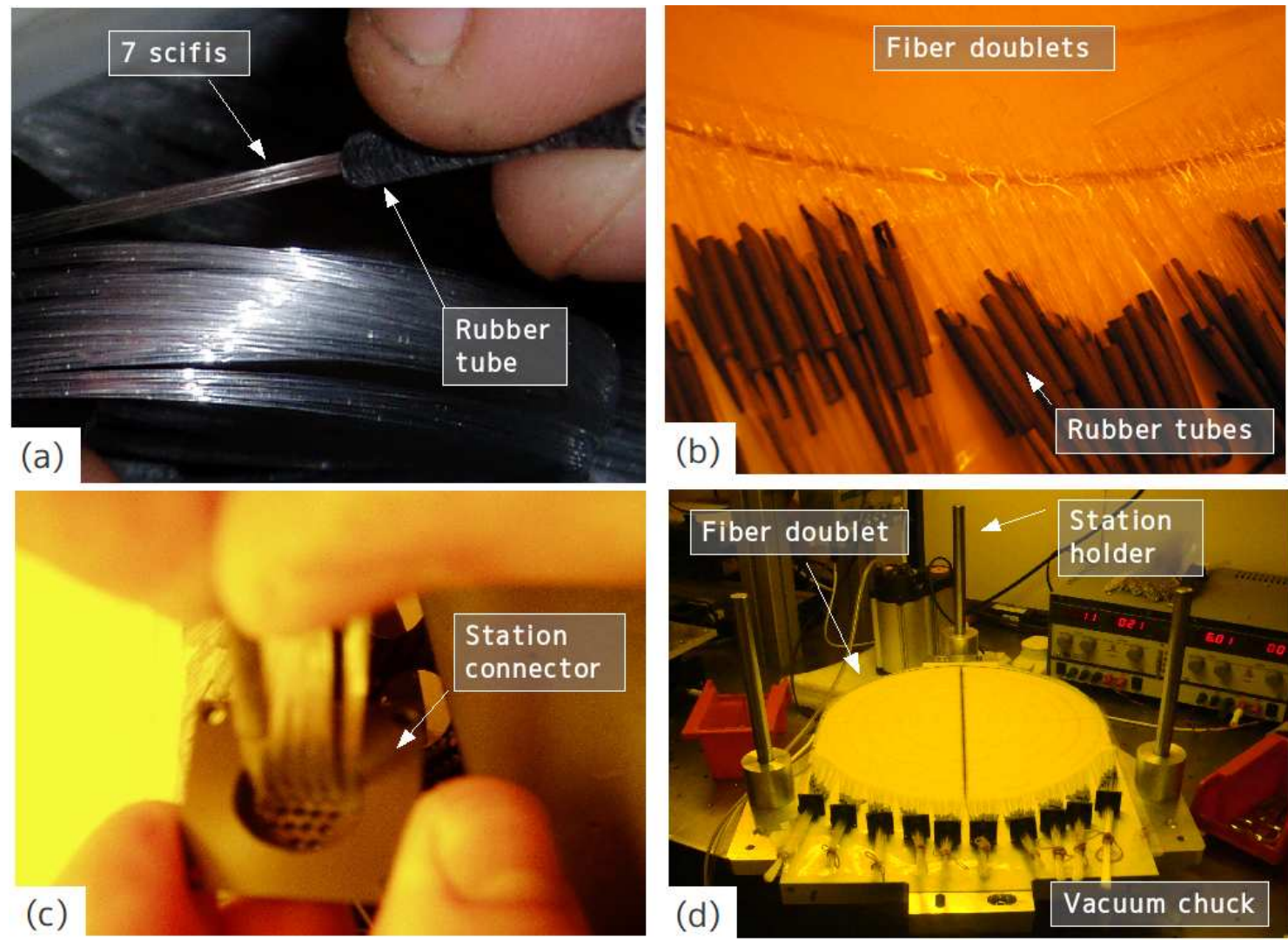

Figure 6.3: The procedures of bundling and connecting are shown. (a) seven scifis are threaded through a rubber tube. (b) fiber doublet bundled. (c) seven scifis bundled with a tube are threaded through a hole on a station connector. (d) fiber doublet with station connectors attached is fixed on the vacuum chuck. 
the doublet (Figure 6.4 (b)) and left until the glue was cured in a day. The procedure was repeated for another two doublets in sequence in the same way as explained above, except for the angle of doublets to be glued. Once three doublets were glued on the station body, the station connectors were attached on the body (Figure $6.4(\mathrm{c})$ ). The excess fibres were cut so that approximately $25 \mathrm{~mm}$ of fiber protruded from the face of the connector. A vacuum cup was then fitted to the connector and potting adhesive was applied from the rear of the connector. The vacuum applied ensured that the adhesive penetrated over whole fiber within the bore. After the adhesive were extruded through the bore, the station was turned over and adhesive was applied on the front face of the connector (Figure 6.5 (a)). This prevents any movement or vibration in subsequent cutting and polishing operations. Once glue were cured, fiber excess and cured adhesive were cut at a distance of 2-3 $\mathrm{mm}$ from the surface of the connector (Figure $6.5(\mathrm{~b})$ ).

Finally, the surface of the connectors were polished using a diamond-tipped tool. Figure 6.5 (d) shows a close-up view of polished fibers observed by a microscope, in which clad layer of fiber is clearly seen.
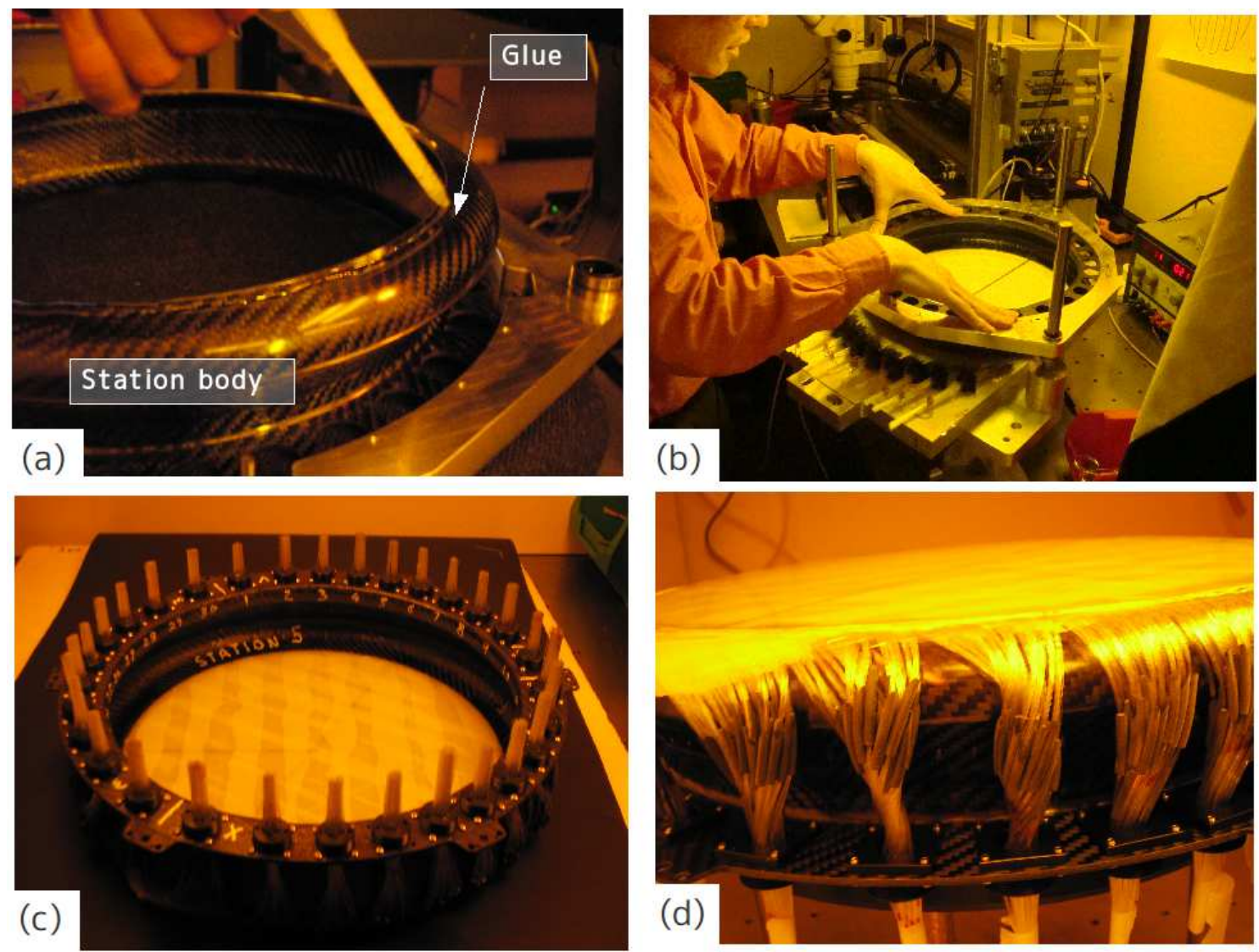

Figure 6.4: (a) Adhesive is applied on the circumference of a station body. (b) A station body with glue applied is put on first scifi doublet which is fixed and aligned to the vacuum chuck. (c) A station body with three scifi doublets glued and optical connectors potted. (d) Photograph showing in detail the fiber run from the double layer to the station connector. 

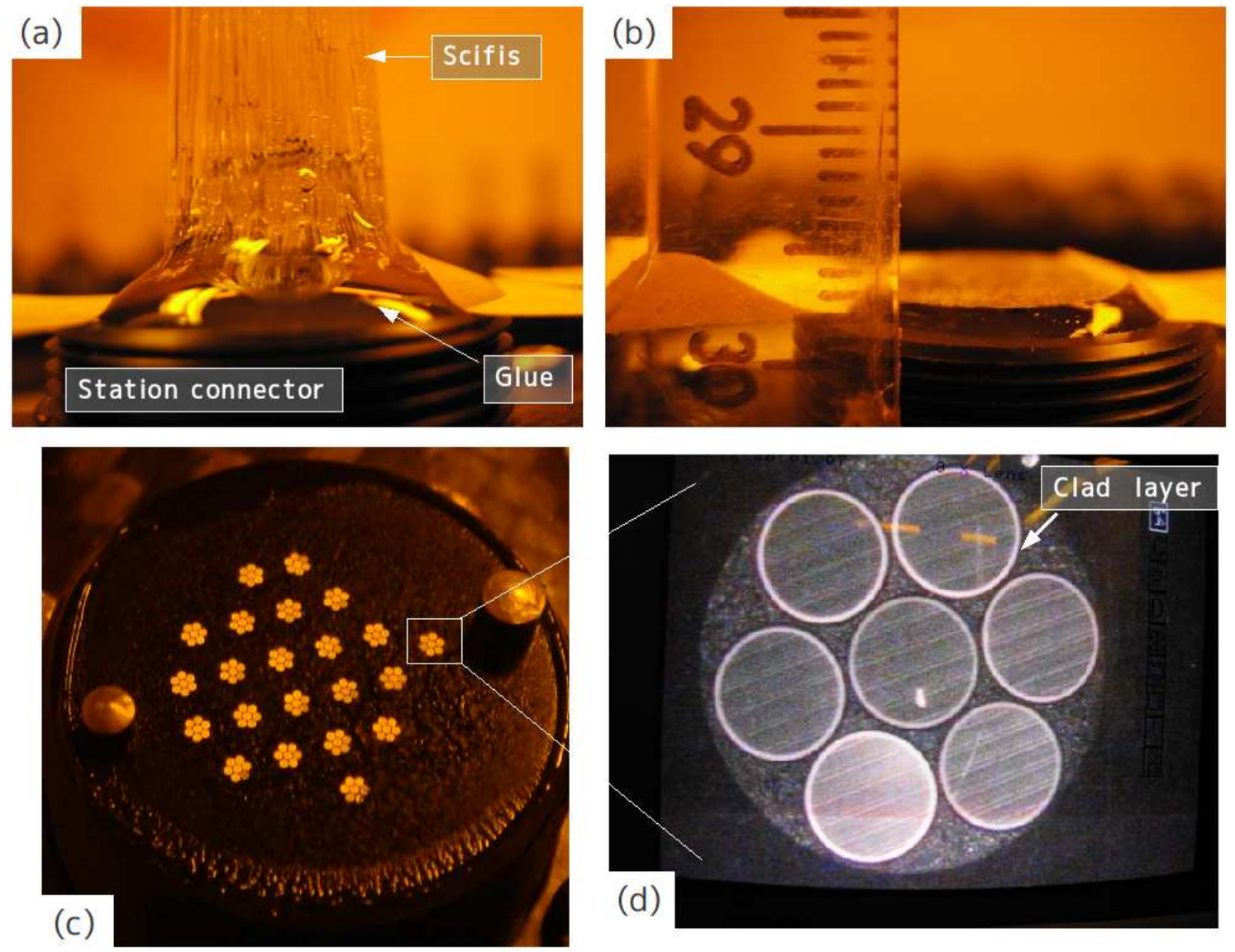

Figure 6.5: (a) A station connector after adhesive is applied. (b) A station connector after excess fibers are cut away. (c) The surface of a station connector after polishing. (d) The microscope view to show seven scifis in one channel. 


\subsubsection{Quality assurance}

Errors on bundling or connecting were checked by illuminating scintillating fibers with LED lights and observing transmitted lights with a CCD camera. The light yield measurement was performed for the produced stations using a ${ }^{57}$ Co gamma-ray source.

\subsubsection{Ensuring of bundling/connecting with a LED-CCD system}

LED scanning was performed twice after the bundling and connector rings to ensure that each of those was correctly operated. After bundling, two items were checked; (i) the number of scintillating fibers in a bundle is seven (ii) the scintillating fibers were correctly bundled without any skips or reverses. On the other hand, after connecting it was checked that each bundles was threaded into the correct channels.

A LED whose emission peak is $405 \mathrm{~nm}$ was used to excite scintillating fibers. The LED was mounted on the top of the structure frame, in which focusing lenses were assembled. The optical system is carried by a linear stage, with a total travel of $750 \mathrm{~mm}$ and a precision of $10 \mu \mathrm{m}$ to scan the fibers. The focused light was directed to the fiber doublet fixed on the vacuum chuck. The transmitted light was recorded by the CCD camera, Toshiba CS8620Ci (Figure 6.6).

The stage was moved at a constant speed of $1,250 \mu \mathrm{m} / \mathrm{s}$ and the images were recorded at 24.98 frame per second (i.e. $50 \mu \mathrm{m}$ per frame). The diameter of the fiber core is $308 \mu \mathrm{m}$ ( $88 \%$ of fiber diameter) and therefore the illumination in a fiber was observed at six frames with a point-like light.

To examine bundles and station connectors by viewing with a camera, a comb with fourteen grooves and a bridge with ten grooves were employed. The arrangement of the bundles in the comb and the station connectors in the bridge are shown in Figure 6.7.

The fiber identification was made by software (Figure 6.10). In Figure 6.11, the identified fibers are represented with different colors. The intensity on each fiber was then estimated by summing intensities of nine pixels around the fiber center.

The measured intensity on each fiber is shown as a function of frame number at top of Figure 6.11. From Figure 6.11, it is seen that illumination in each fiber is found to spread in about ten frames for the top fibers, and twenty frames for the bottom fibers. Also, it is seen that the intensity at bottom fibers is about half of that for top fibers. This is caused by the light absorption by the top fibers and adhesives in between.

To investigate the above items, a peak of the frame number was estimated for each fibers, which was calculated by averaging the frame numbers where the intensity became half of the maximum. The bundle numbers are shown as a function of peak values at bottom of Figure 6.11. From this, it is found that the number of fibers in bundles are seven and no skipping or reversing are identified.

The same scanning and analysis were carried out after the bundles were connected into holes on the station connectors.

\subsubsection{Light-yield measurement with a ${ }^{57}$ Co radio-active source}

Light yields of the stations built were measured with VLPCs using a ${ }^{57}$ Co radioactive source, emitting 122-keV gamma-rays, at Imperial College London. The VLPCs were cooled by a twocassette cryostat with a cryocooler, which was the same as used at the beam test at KEK (Figure 6.13 (a)). Two AFEIIt boards (1024 channels) were used for the test. Five external light-guides were attached at the D0 warm ends on the cryostat with optical grease applied and covered with 

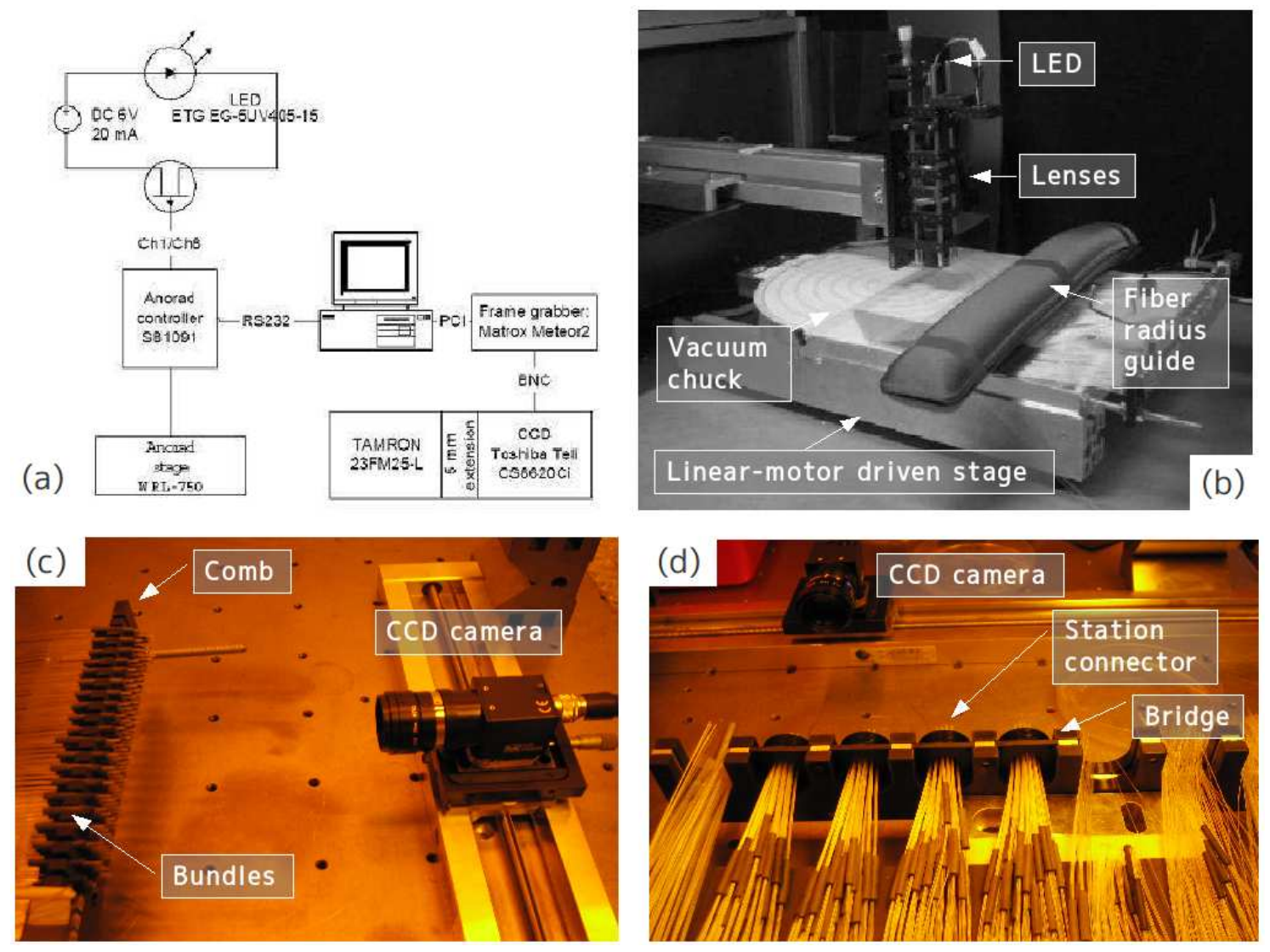

Figure 6.6: (a) Schematic diagram of the QA system. (b) Photograph of the station assembly QA setup. The optical system is attached to a linear stage and held over the plane of scifi doublet to illuminate the channels in sequence. (c) Photograph showing bundles aligned in the comb are set in front of CCD camera. (d) Photograph showing station connectors in the bridge are faced with the CCD camera. 

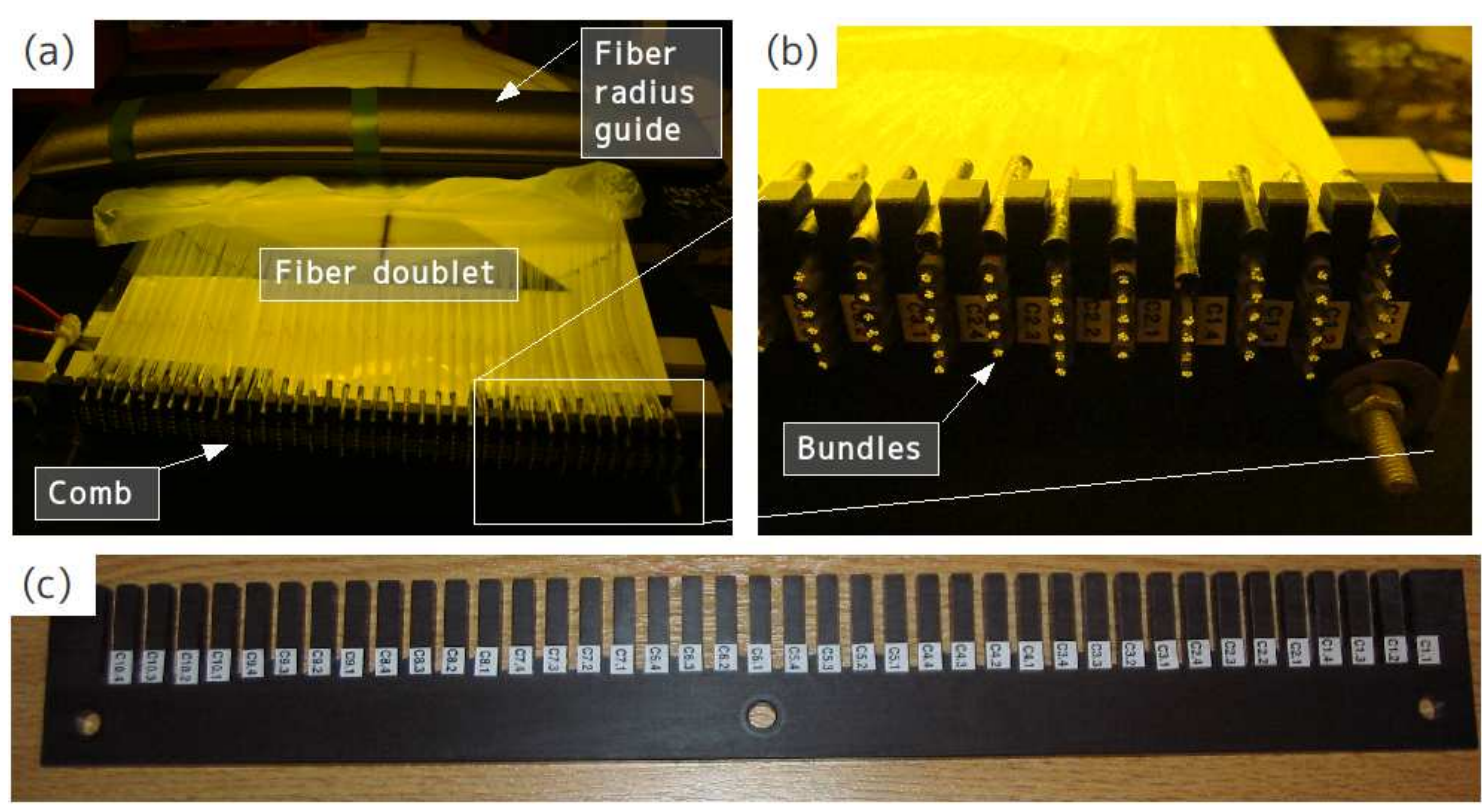

Figure 6.7: (a) Fiber doublet and the comb located on the vacuum chuck. (b) Close-up view of the comb. (c) The comb used for the QA. There are fourteen grooves to stack fiber bundles.
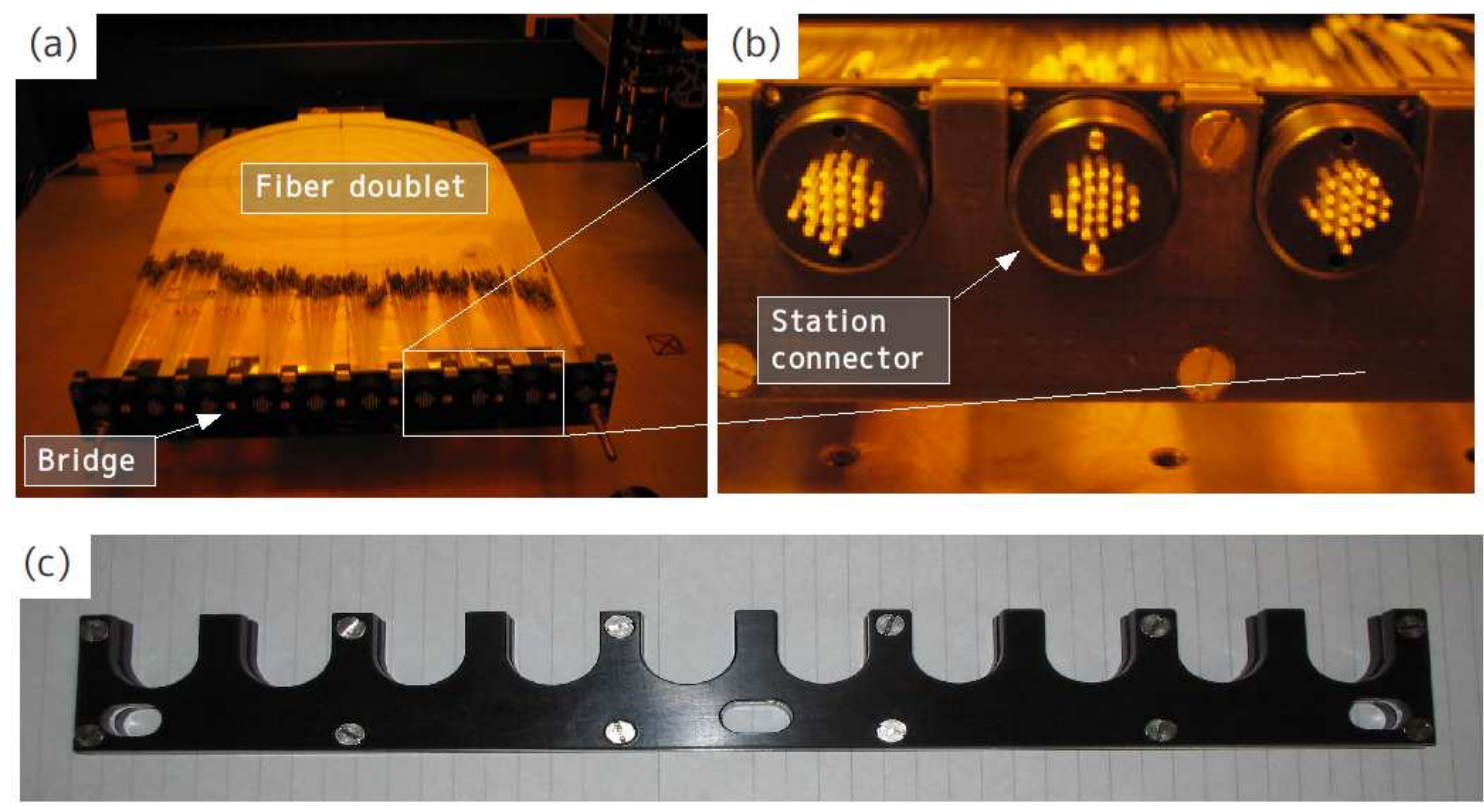

Figure 6.8: (a) Fiber doublet and the bridge on the vacuum check. (b) Close-up view of the bridge. (c) The bridge used for the QA. There are ten grooves to put station connectors. 


\section{Arrangement of fiber bundles in the comb}
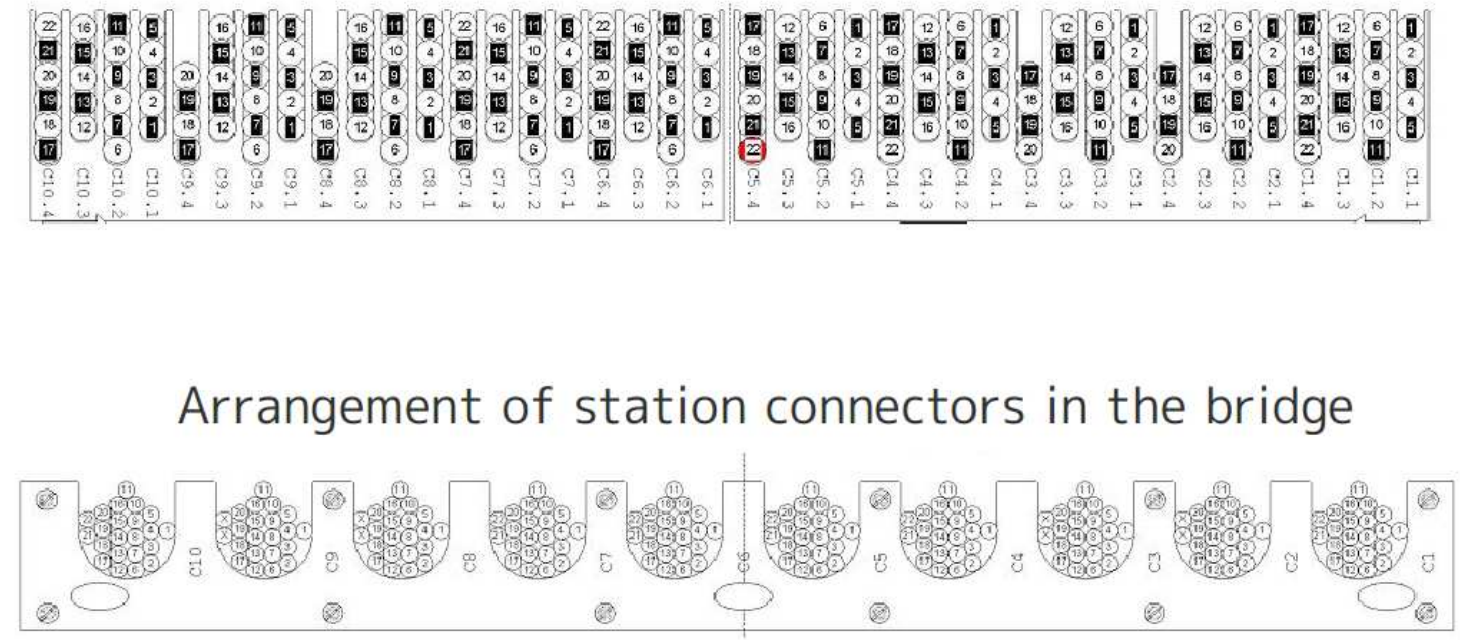

Figure 6.9: (a) Arrangement of fiber bundles in the comb in which fourteen grooves are made. Fiber bundles stacked in grooves are described. (b) Arrangement of station connectors in the bridge. Station connectors put on grooves are described.

a black-out sheet. The other ends were connected with bulkhead connectors of the internal lightguides with optical grease applied.

Five internal light-guides, six station stands to hold a station body and a $\mathrm{x}-\mathrm{y}$ linear guide were set in the QA box (Figure $6.13(\mathrm{~b})$ ). The station to be tested was put on the stands and fixed with screws. The height of the stand was adjusted to be about $15 \mathrm{~cm}$ to make sufficient distance from the bottom not to damage light-guide during the procedure of attaching light-guides. Station connectors were attached to the station with optical grease applied (Figure 6.13 (c)). An arm, on the top of which the source was mounted, was fixed with the linear guide so as to make the distance of $1 \mathrm{~cm}$ from the surface of the station (Figure $6.13(\mathrm{~d})$ ). In order to illuminate all the scintillating fibers, total 28 scanned positions were made (Figure 6.12). The location of the source was determined by the $\mathrm{x}$ and $\mathrm{y}$ positions of the guide which were controlled from the PC.

Data was taken with a self trigger by $50 \mathrm{k}$ events at each point. The trigger rate was $38 \mathrm{~Hz}$. It took eleven hours to complete QA for one station from assembly to measurements of light yields.

Light-yields for fibers, which was under the source, were calculated in the same way as described in section 5.3.3. A typical light-yield distribution is shown in Figure 6.14. The peak seen around at 11 p.e. is considered as the signals by cosmic-rays. Events triggered by $122 \mathrm{keV}$ gamma-rays (compton edge is around 9 p.e.) are considered as small compared with cosmic-rays. The peak seen around at 3 p.e. is considered as VLPC thermal noises (see Figure 6.15). The light-yields as a function of the fiber number for the station No.5 is shown in Figure 6.16. Differences of light-yields seen in Figure 6.16 can be caused by that of the transmission in the light-guides, since the same structure was seen in other stations. The quality assurance was not applied for the light-guides (serial numbers are from No.1 to No.5) used for that test, therefore, some channels of poor transmission could be included in those light-guides. Table 6.1 summarizes the fitted means averaged in each view for the stations used for the trackers of No.1 and No.2. In all the views, light-yields of more than 10 photo-electrons were observed. 

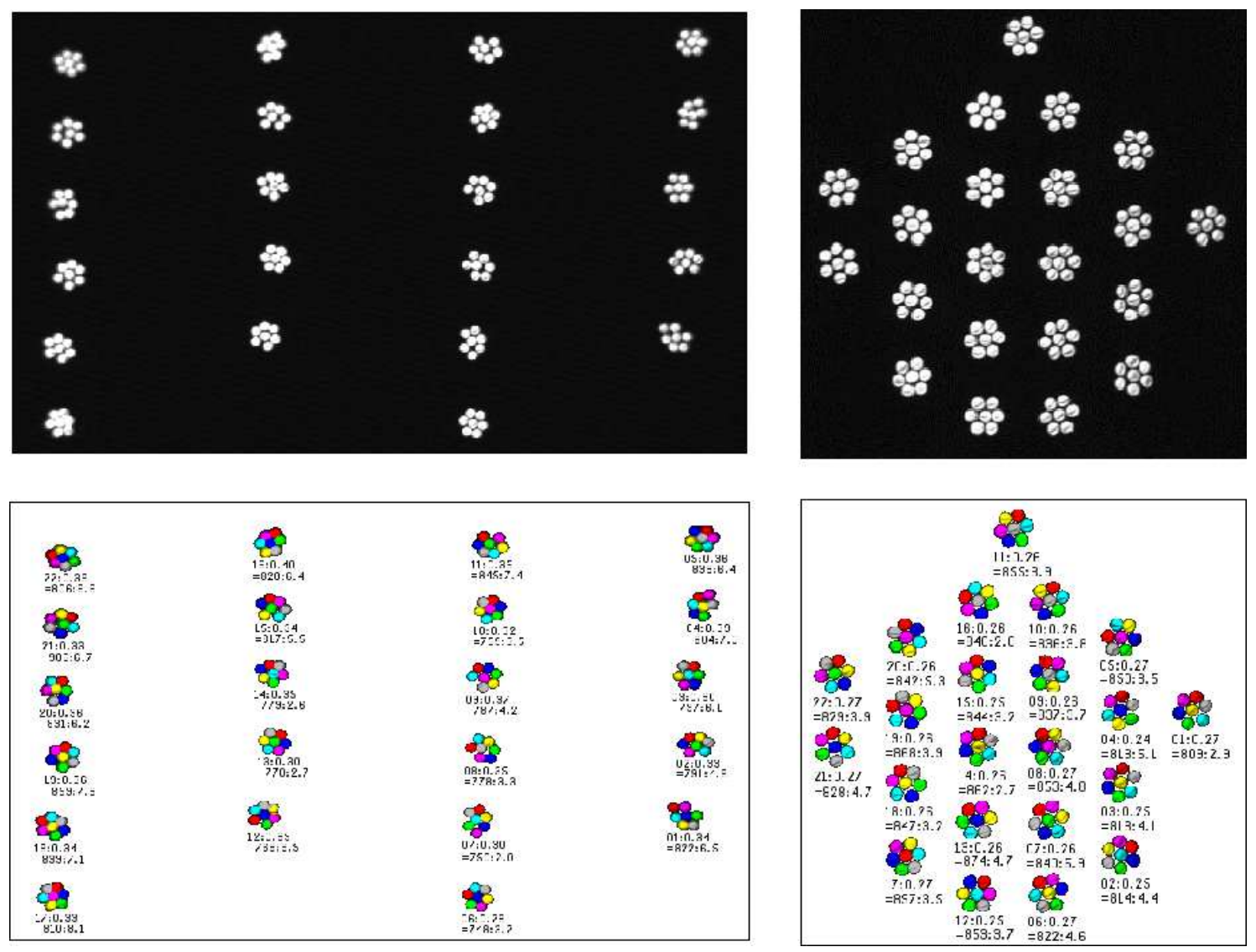

Figure 6.10: CCD images of bundles in the comb (top left) and a station connector in the bridge (top right). In bottom, scifis in each bundle identified by software are shown with seven colors.

\begin{tabular}{l|c|c|c}
\hline Station & $\mathrm{V}$ & $\mathrm{X}$ & $\mathrm{W}$ \\
\hline 5 & 10.95 & 12.36 & 11.29 \\
6 & 10.81 & 12.32 & 11.43 \\
7 & 10.91 & 12.15 & 11.38 \\
9 & 10.77 & 12.06 & 11.21 \\
10 & 10.81 & 11.99 & 11.28 \\
11 & 10.78 & 12.01 & 11.31 \\
12 & 10.89 & 12.04 & 11.27 \\
13 & 10.88 & 12.04 & 11.23 \\
14 & 10.73 & 12.06 & 11.22 \\
15 & 10.70 & 12.03 & 11.18 \\
\hline
\end{tabular}

Table 6.1: Result of light-yield measurement for stations used for the tracker No.1 and No.2. First column indicates the serial number of stations. The average of fitted means in $\mathrm{V}, \mathrm{X}$ and $\mathrm{W}$ view are shown in the following columns, respectively. 

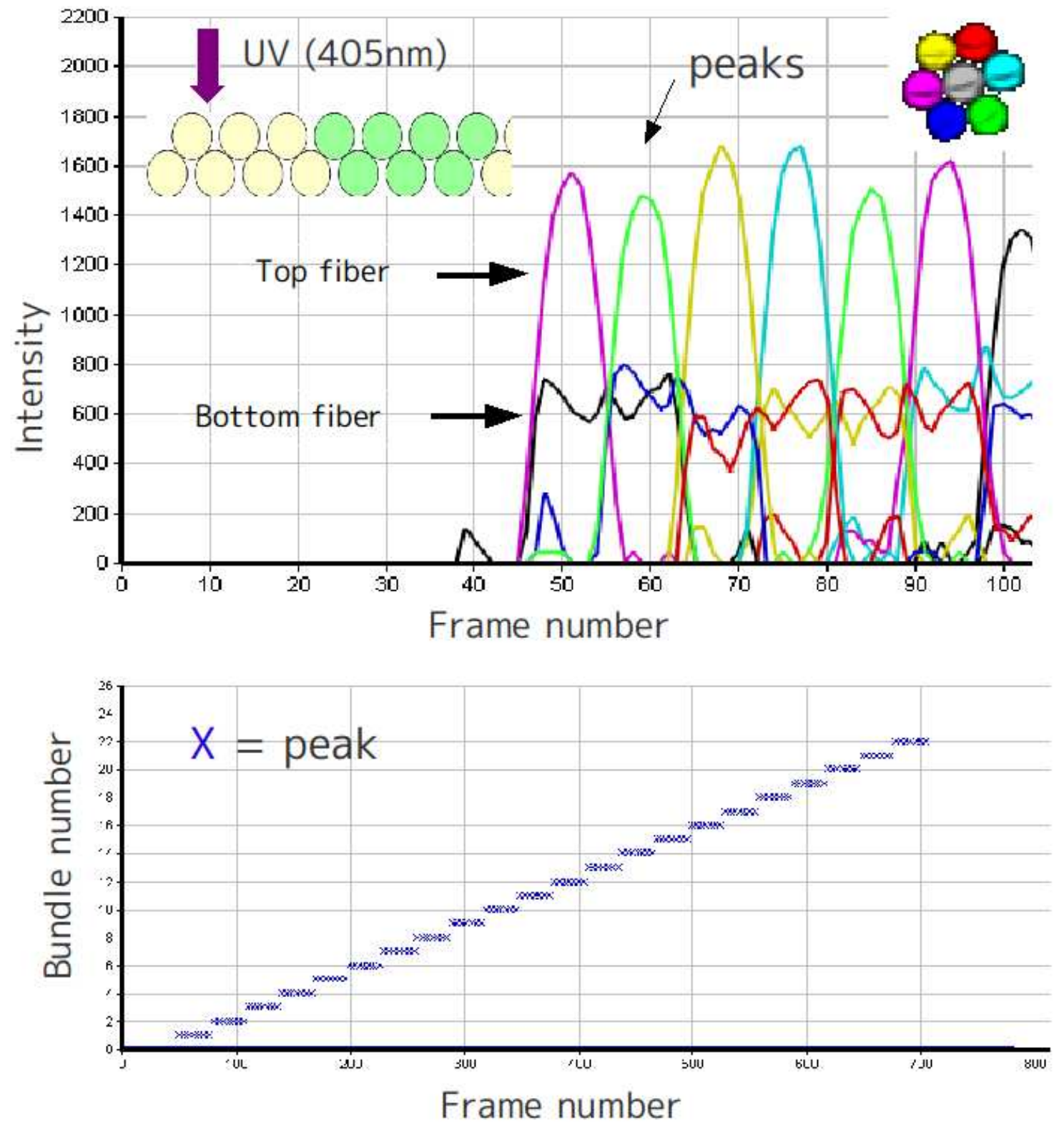

Figure 6.11: Measured intensity versus frame number (top). The peak is calculated by averaging frame numbers where intensities are half of the maximum in the fiber. The bundle number versus the peak value (bottom). In each bundle, seven scifis are observed and no skipping or swapping are found. 


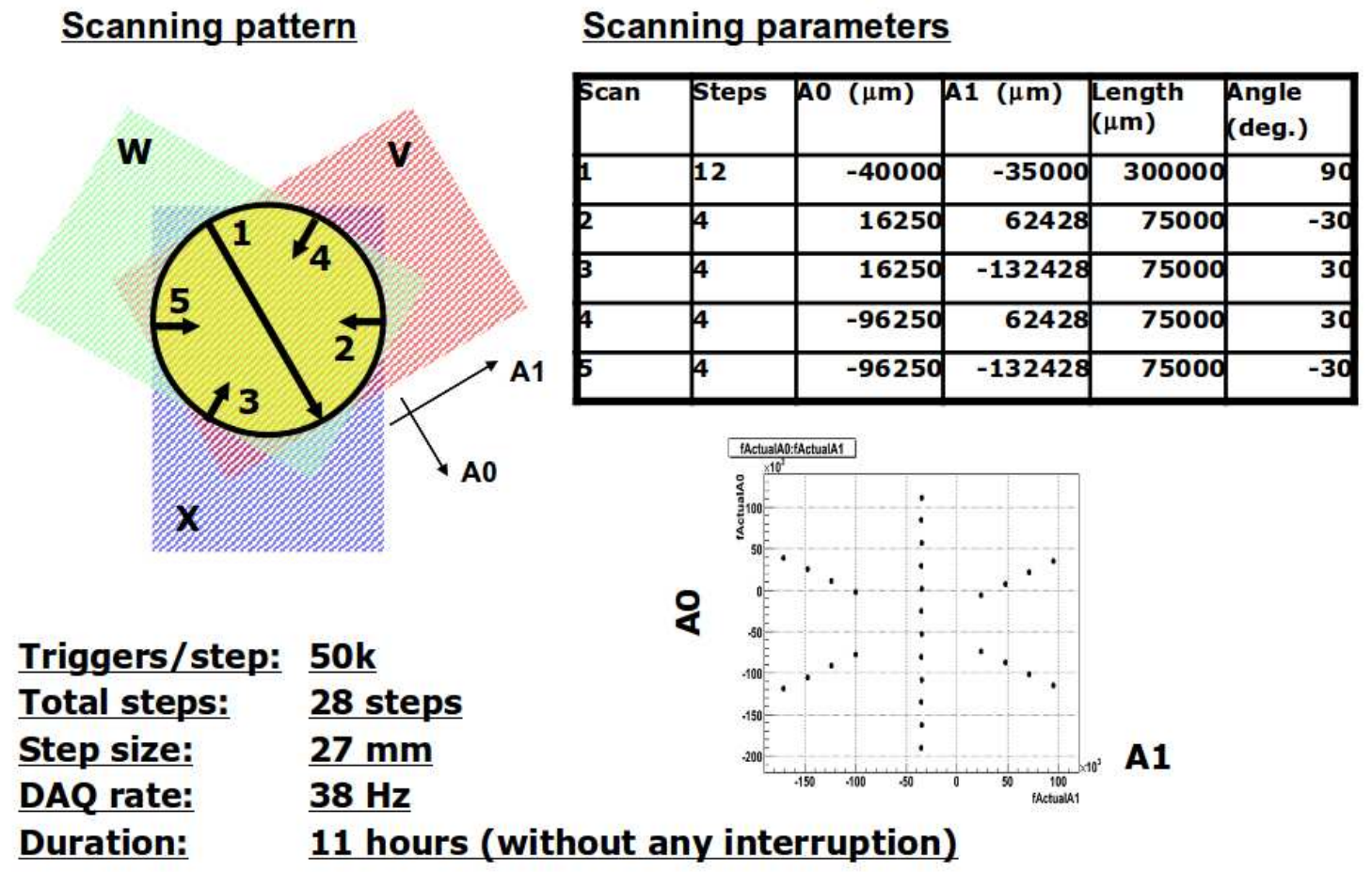

Figure 6.12: Drawings showing scanning pattern applied at station QA.

\subsection{Light-guides}

Light-guides used for the MICE SciFi trackers were designed at Osaka University, Japan. The lightguides were fabricated at the G-tech company, Japan. Quality Assurance (QA) procedures were applied to light-guides fabricated at Osaka University. Light-guides were polished at the FNAL and then shipped to the RAL to perform the cosmic-ray test. In the following sections, the processes of making light-guides and procedures of quality assurance for light-guides are described.

\subsubsection{Fabrication}

Four-meter lengths of clear fibers were cut with a margin of $10 \mathrm{~cm}$ from the spool on which they were delivered by the manufacturer (Kuraray). First, good clear fibers were selected (see below). Clear fibers of good qualities were then cut into two, one is for the internal and the other is for the external. Clear fibers were threaded in holes on the optical connectors at the both ends. Potential errors on threading into wrong holes were checked once a gluing was finished (see below). The lightguides were polished briefly to measure transmittances of clear fibers at Osaka University, Japan. Figure 6.17 shows the process of assembling of an internal light-guide in the G-tech company, Japan. Light-guides were shipped to the FNAL to apply final polishing by a diamond cutter.

These processes will be described in detail as follows. 

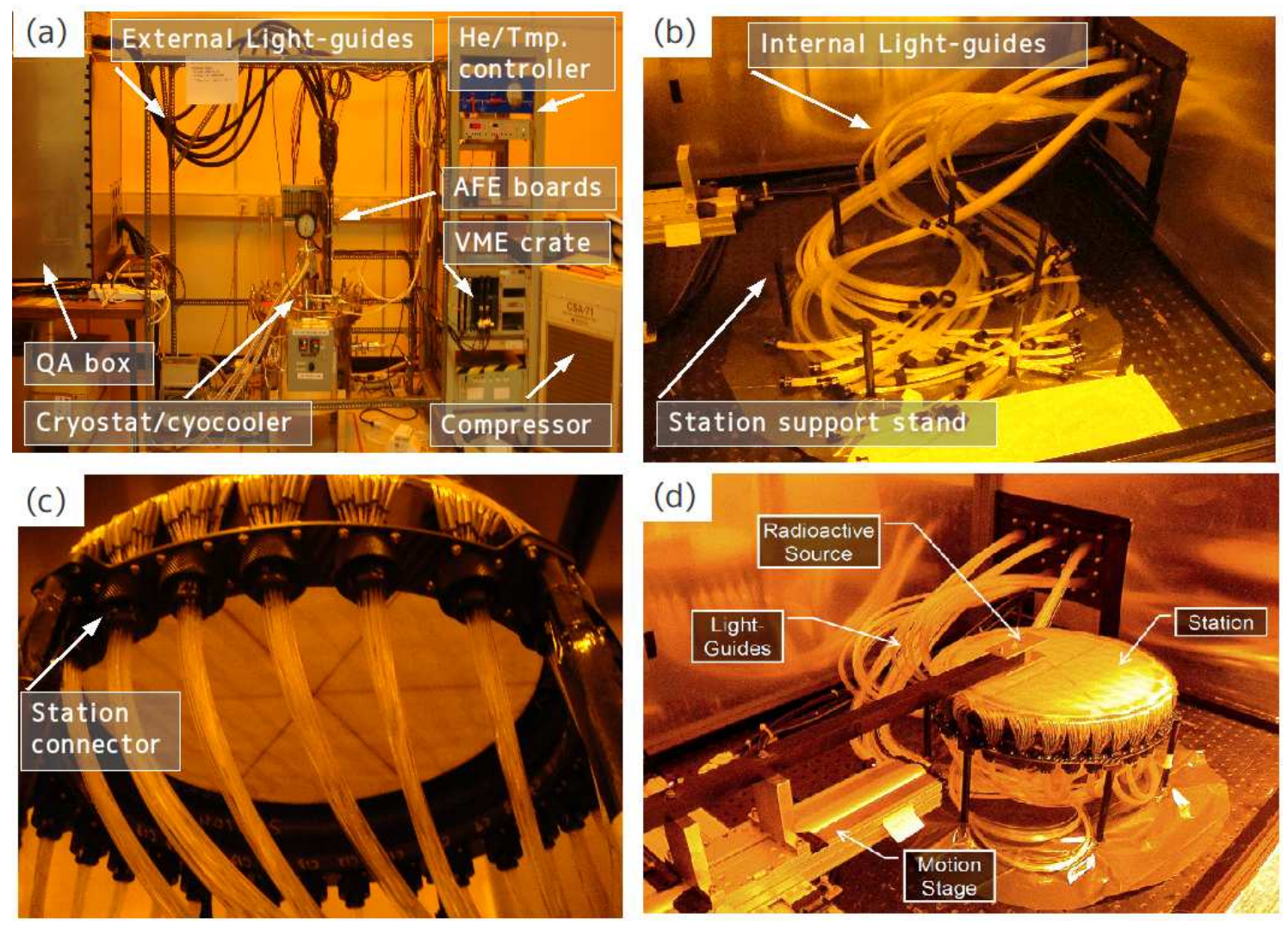

Figure 6.13: (a) Setup of the light-yield measurement in the lab at the Imperial College London. In the middle, the two-cassette VLPC cryostat with cryocooler is placed. The cryocooler and the compressor, shown in the right, are connected by two tubes in which helium is supplied or returned. On the cryostat, two AFEIIt boards are mounted. External light guides are attached on the warm end of the cryostat. The station to be tested is set in the QA box shown in the left. A patch pannel used when vacuuming cryostat, a temperature controller and a VME crate are located in the rack shown in the left. (b) Inside view of the QA box before mounting a station on support stands. (c) A station mounted on the support stands after light-guides are attached (viewed from the bottom). (d) Completed setup for the measurement. A ${ }^{57}$ Co radio-active source is mounted on top of an arm, from which collimated gamma-rays of $122-\mathrm{keV}$ are emitted toward the station. 


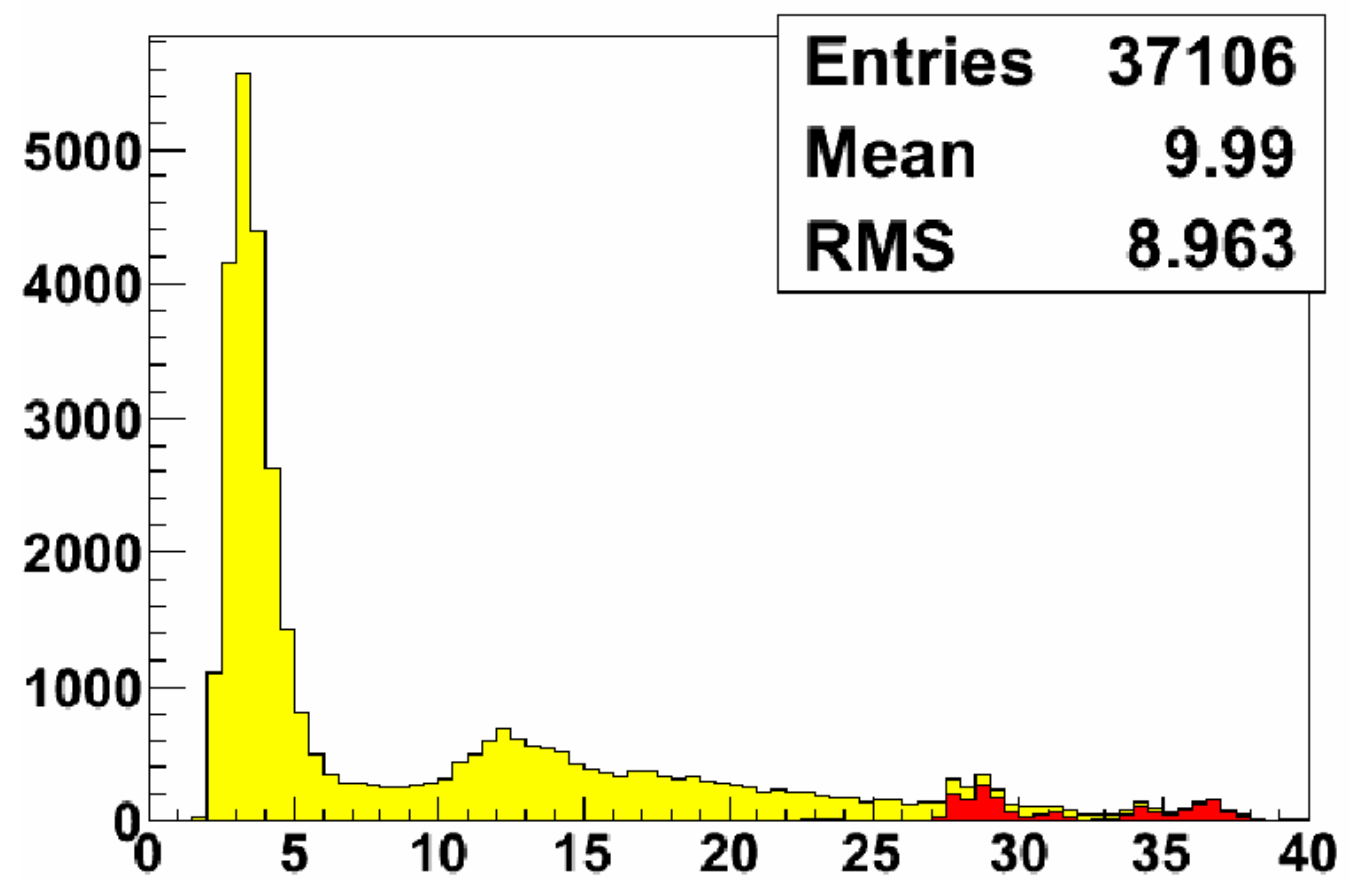

Figure 6.14: A typical light-yield distribution observed at fiber under the source. The peak around at 12 p.e. is considered as cosmic-rays and the peak around 3 p.e. is considered as VLPC thermal noise. The amount of events by $122 \mathrm{keV}$ gamma-rays are considered as small compared as cosmicrays (compton edge is around 9 p.e.). The red hatched histogram corresponds to ADC-saturated events.

\begin{tabular}{ccccc} 
Serial Number & Tracker & Station & Internal & External \\
\hline $6-10$ & 1 & 5 & $1500 \mathrm{~mm}$ & $2500 \mathrm{~mm}$ \\
$16-20$ & 1 & 4 & $1950 \mathrm{~mm}$ & $2050 \mathrm{~mm}$ \\
$21-25$ & 1 & 3 & $2250 \mathrm{~mm}$ & $1750 \mathrm{~mm}$ \\
$26-30$ & 1 & 1 & $2600 \mathrm{~mm}$ & $1400 \mathrm{~mm}$ \\
$31-35$ & 1 & 2 & $2400 \mathrm{~mm}$ & $1600 \mathrm{~mm}$ \\
$36-40$ & 2 & 5 & $1500 \mathrm{~mm}$ & $2500 \mathrm{~mm}$ \\
$41-45$ & 2 & 4 & $1950 \mathrm{~mm}$ & $2050 \mathrm{~mm}$ \\
$46-50$ & 2 & 3 & $2250 \mathrm{~mm}$ & $1750 \mathrm{~mm}$ \\
$51-55$ & 2 & 2 & $2400 \mathrm{~mm}$ & $1600 \mathrm{~mm}$ \\
$56-60$ & 2 & 1 & $2600 \mathrm{~mm}$ & $1400 \mathrm{~mm}$ \\
\hline
\end{tabular}

Table 6.2: List of light-guide length. Serial number is the number marked at connectors. Tracker 1 is for upstream and 2 for downstream. Station 5 is located nearest from patch panel, and station 1 is farthest from patch panel. 


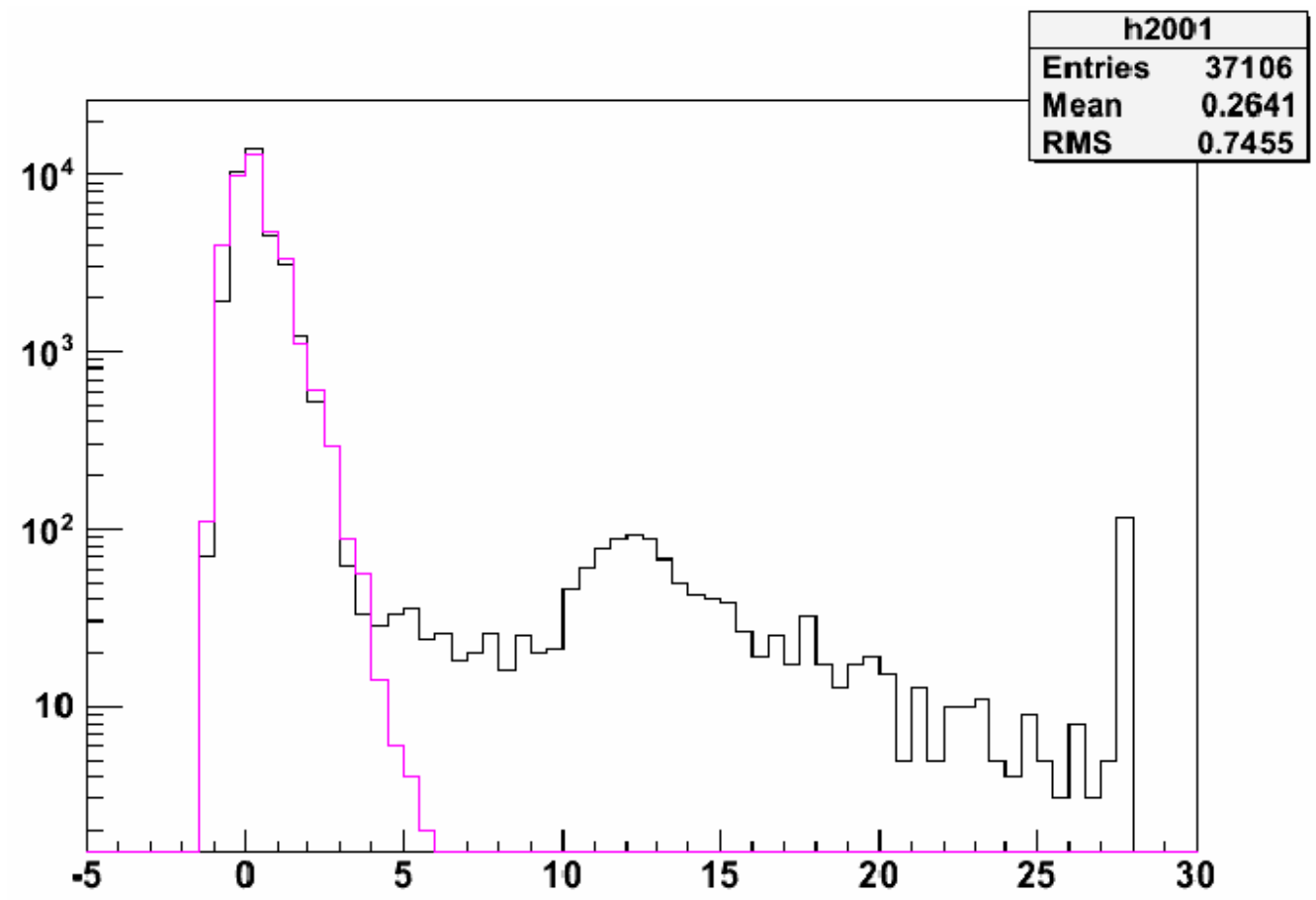

Figure 6.15: Light-yields distributions of a fiber under the source (black) and the apart from the source (light purple). Signals below 5 p.e. are considered as VLPC thermal noise.

\subsubsection{Good-fiber selection}

Good clear fibers were selected in the following way. A bundle of 225 fibers of four meters was connected with cookies (15x15 holes) at each end and then glued.

First, possible presence of kinks or cracks produced in handling the light-guides during manufacture were examined. The surface of each connector was exposed to a strobe light and reflected lights were recorded in a photograph taken with a digital camera. An example of such a photograph, showing evidence of a damage in one fiber, is shown in Figure 6.18. The damaged channel can be clearly identified in the photograph where the injected light is reflected from the damage position in the fiber.

Next, the transmittance of clear fibers were measured and the fibers whose transmittances were lower than $90 \%$ were rejected. Details of transmittance measurement are described in the section below.

Finally, cookies were cut off. Bad fibers found at these test were further investigated to search for cracks in fibers. When cracks were found in fibers, the fibers were cut off to remove those cracks. Those shorten fibers were re-used to make spare light-guides.

\subsubsection{Assembling}

Using good clear fibers, internal and external light-guides were produced. For internal light-guides, a locking ring was threaded to a bundle of clear fibers. Then clear fibers were threaded one by one in their holes on the optical connectors at both ends. The errors on threading wrong holes were later investigated by the test described in below. 
Station 5

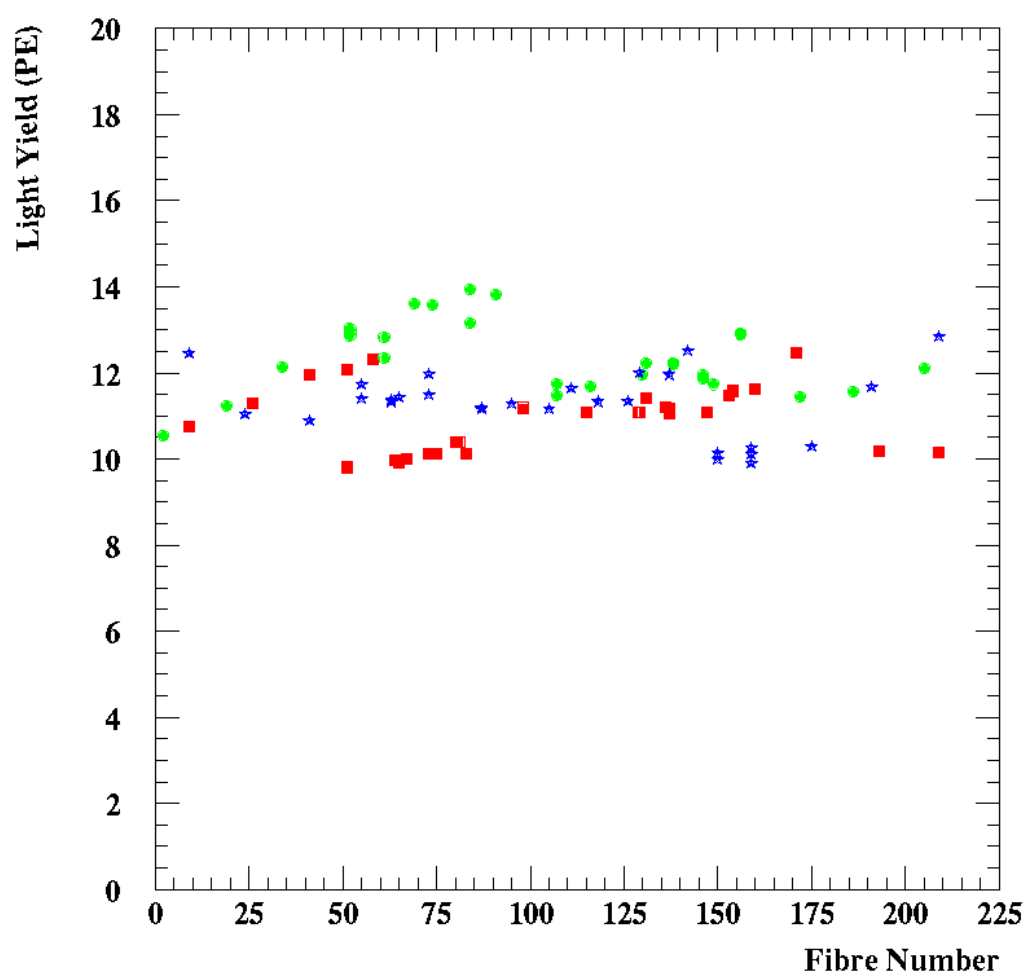

Figure 6.16: Fitted means versus fiber number for the station No.5. In each view, fitted mean of light yield in a fiber under the source are plotted. The variety of light yield found in each view is considered as that of transmission in light-guides as explained in text. 

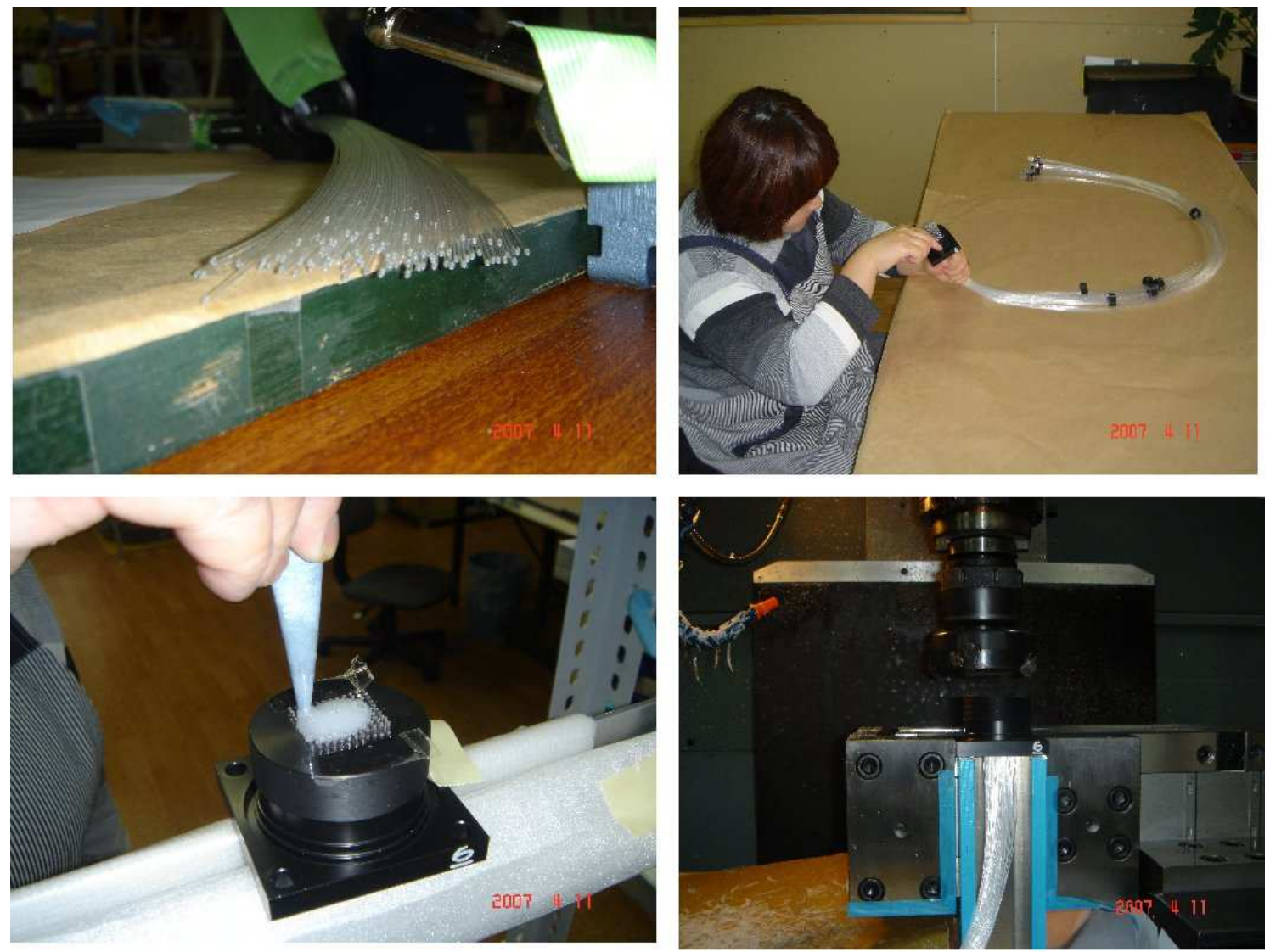

Figure 6.17: Photograph of an assembling of an internal light-guide at the G-tech company, Japan. A bundle of fibers used for one light-guide is shown (top left). An internal light-guide is being attached with connectors (top right). A glue is applied on a bulkhead connector (bottom left). A glued connector is being polished (bottom right).

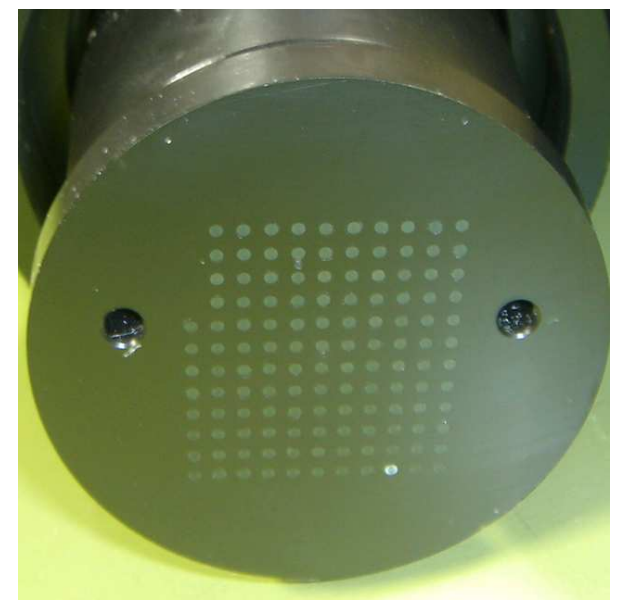

Figure 6.18: Picture of a bulkhead connector taken at the reflection-light test. A reflected light is clearly seen in a channel. 
For external light-guides, flexible tubes and heat-shrink tubes are threaded to a bundle of clear fibers. The optical connectors and those adjacent connectors were assembled and glued. Then clear fibers were threaded one by one in their holes on optical connectors as internal light-guide. Heat-shrink tubes were finally heated after a gluing was finished.

\subsubsection{Gluing}

A first gluing was applied from the rear of the connector. The gluing was fluid enough to run through a hole so that the vacuum suction was not applied. The gluing was cured in a day. A second gluing was applied from the font of the connector to ensure that fibers to be cut away were fixed tightly not to vibrate and damaged during polishing.

\subsubsection{Polishing}

After the glue was cured, the connectors were polished briefly at G-tech company. Then transmittance measurement for light-guides were performed at Osaka University. Finally, light-guides were polished with diamond cutter at the FNAL.

\subsubsection{Quality assurance}

The quality assurance procedures were applied for light-guides in the following way. The transmittances of clear fibers were measured (see below). The total number of channels to be test was 15,744 and that of channels whose transmittances were lower than $90 \%$ was 69 , or $0.4 \%$ of the total.

The assignment of clear fibers (mapping) was then ensured (see blow). The errors on the mapping were found at 4 out of 123 light-guides, which is used for the analysis.

\subsubsection{Transmittance measurement with a LED-CCD system}

The bundle of fibers was illuminated using a diffuse source of green light (see Figure 6.19). The LED and diffusers were installed in the LED box. Central lights in the region of $6 x 6 \mathrm{~cm}$ were used to get an uniform amount of light, The variation in light transmittance from fiber to fiber was measured to be less than $10 \%$. The optical connector was locked with the LED box using a jig. Three kinds of jigs were prepared for a test cookie, external (D0) and internal connector (3A). In the effective area of $6 \times 6 \mathrm{~cm}$, only four connectors out of six were attached at a time, therefore two measurements were made for an internal light-guide. The light transmitted along the light-guide was observed with a CCD camera, OLYMPUS E-10. An optical connector at the other end was fixed with a support jig. Two kinds of support jigs were prepared for test cookie and external (2A) or internal (1A) connector. The CCD camera was located apart from the connector as close as possible to get a focus. The connector attached with a support jig and the camera were mounted on the same board and shielded from the room light using a light-tight box. The light-guide and the LED box were shielded using a black-out sheet.

A sample image taken with the camera is shown at left-bottom in Figure 6.19. An images was recorded with the format, ORF (Olympus Raw image Format). Intensities of the green component for all pixels were extracted from a raw image. A pedestal was calculated by averaging intensities in the image. Then, for each channels, square regions were defined and pedestal-subtracted intensities in these regions were summed to estimate light-yields (see bottom right in Figure 6.19)

. The transmissions were calculated by normalizing the light-yields with the peak value which was 
calculated by fitting with the Gaussian distribution. The transmissions for internal and external light-guides are shown in Figure 6.19. From the result, the portion of channels whose transmissions are smaller than $90 \%$ is $0.4 \%$.

\subsubsection{Ensuring of channel assignment}

The ensuring of channel assignment was made in the following way. From surface of a connector a light was injected using a piece of clear fiber channel by channel from No.1 to No.128. The moving image was recorded by a camera to check that all of channels were shined in the expected order. The assignment of channels (mapping) were established by examine the image. The mapping errors found at four light-guides. The details are as follows. The channel No.109 and No.110 in internal No.6 are swapped. The channel No.65 and No.76 in internal No.10 are swapped. The channel ordering is reversed (No.1 for No.128 etc.) in External No.30 and No.56.

\subsection{Tracker assembling and installation}

The installation of the tracker into the spectrometer solenoid bore will have to be carried out in UV-filtered light to avoid any degradation of the scintillating fibers.

When on the platform, the frame that will support the internal light-guides during the installation will be attached to the tracker via a flexible coupling to ensure that no stresses are transferred to the tracker during the fitting of the internal light-guides (Figure 6.23). The light-guide connections will be checked to ensure that the light-guides are correctly positioned and labelled.

With the internal light-guides attached, the tracker assembly (consisting of the tracker, the lightguide support, and the light-guides) is ready for installation. If necessary, the tracker assembly can be stored in an extended light-tight storage tube for installation at a later date. For cosmic-ray tests, the storage tube will be used as light-tight box.

For installation, the complete tracker assembly is slid into the solenoid bore to the correct $\mathrm{z}$ position. The tracker will sit on four adjustable feet, two at each end. The adjustable feet will be used to align the tracker with the magnetic axis of the solenoid. Once this has been done, the location bracket will be fitted. The location bracket locks the tracker in the $\mathrm{z}$ and azimuthal positions (Figure 6.24).

The light-guides will now be removed one-by-one from the support frame and fitted into the patch panel. This is a procedure that requires great care to ensure that the fiber are not damaged and a collar will be fitted to ensure that the bore is kept clear (Figure 6.26). Finally, the patchpanel cover will be fitted and, if the external light-guides are to be fitted at a later stage, the patch panel connectors will have their light-tight caps fitted. 

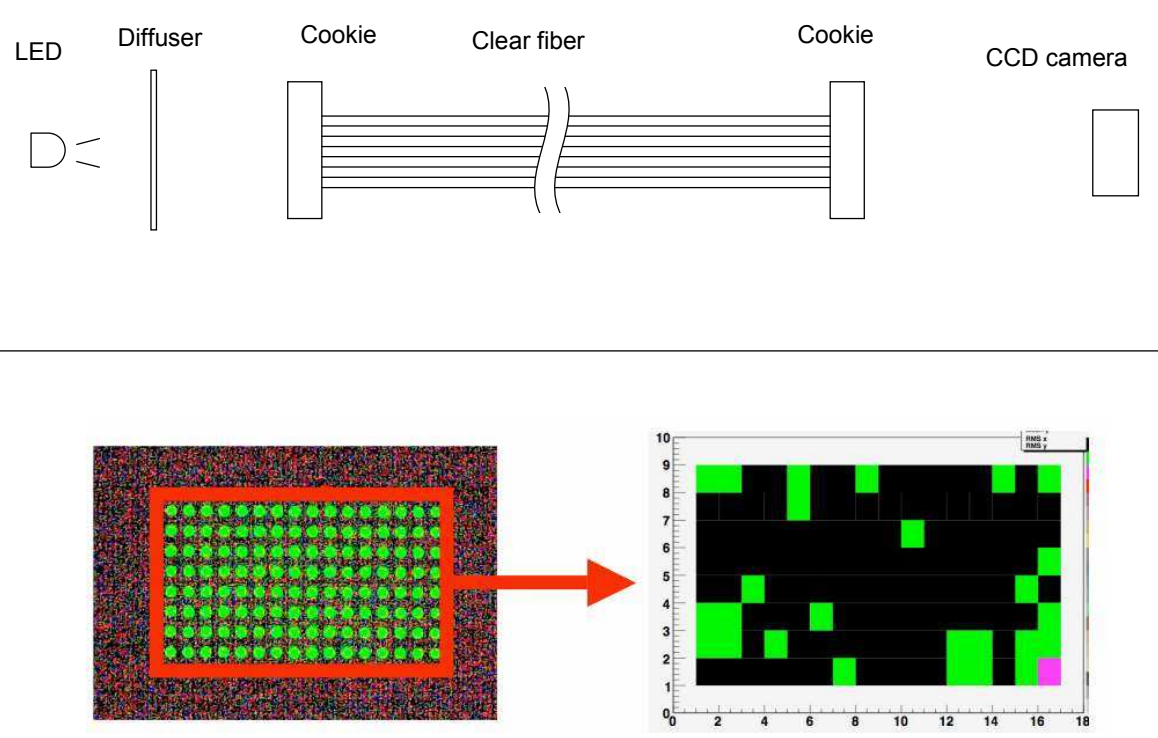

Figure 6.19: Schematic illustration of the setup for the transmission test is shown (top). The LED and diffuser was installed in the LED box (not shown in this picture). The connector on the left hand was fixed with the LED box using a jig. The connector on the right hand was fixed with a support jig. The connector fixed with the support jig and a CCD camera were set in a light-tight box. A sample image taken by the CCD camera is shown (left-bottom). Green-light spots are seen in the middle. The light-yield for these channels are calculated (right-bottom).

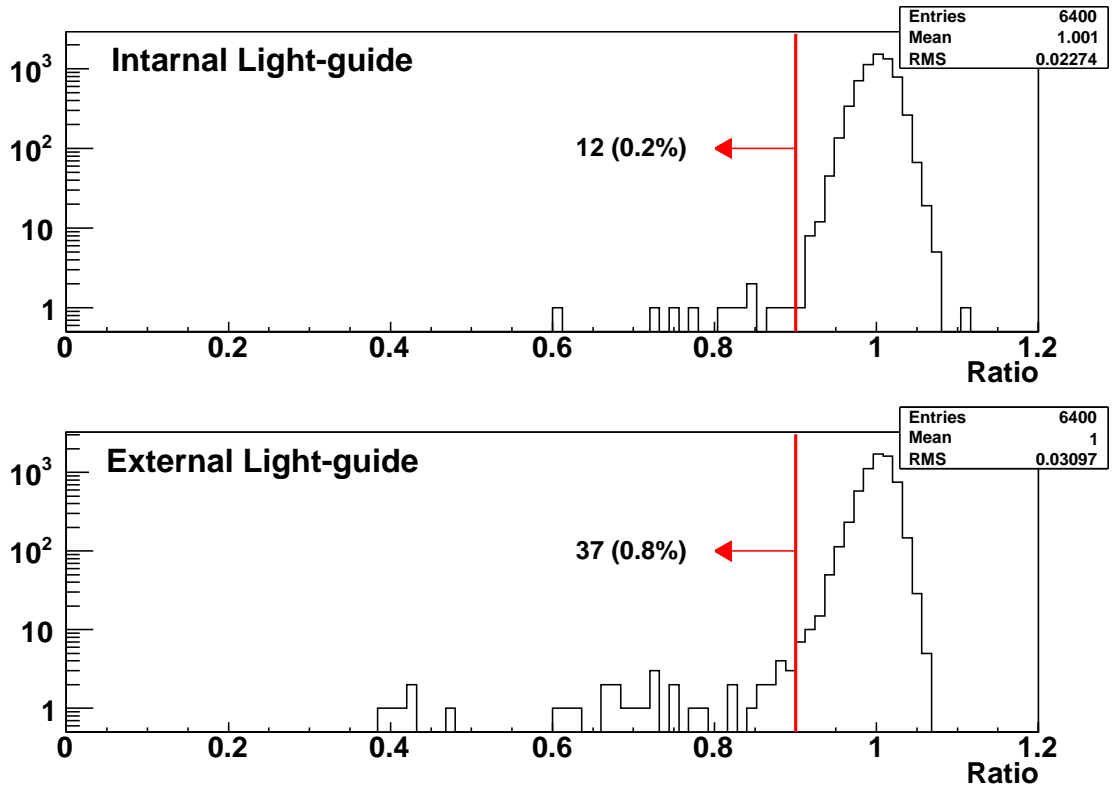

Figure 6.20: Transmission histograms measured by the test for internal (top) and external (bottom) light-guides. The ratio of channels whose transmissions are smaller than $90 \%$ is $0.2 \%$ for internal light-guide and $0.8 \%$ for external one. 


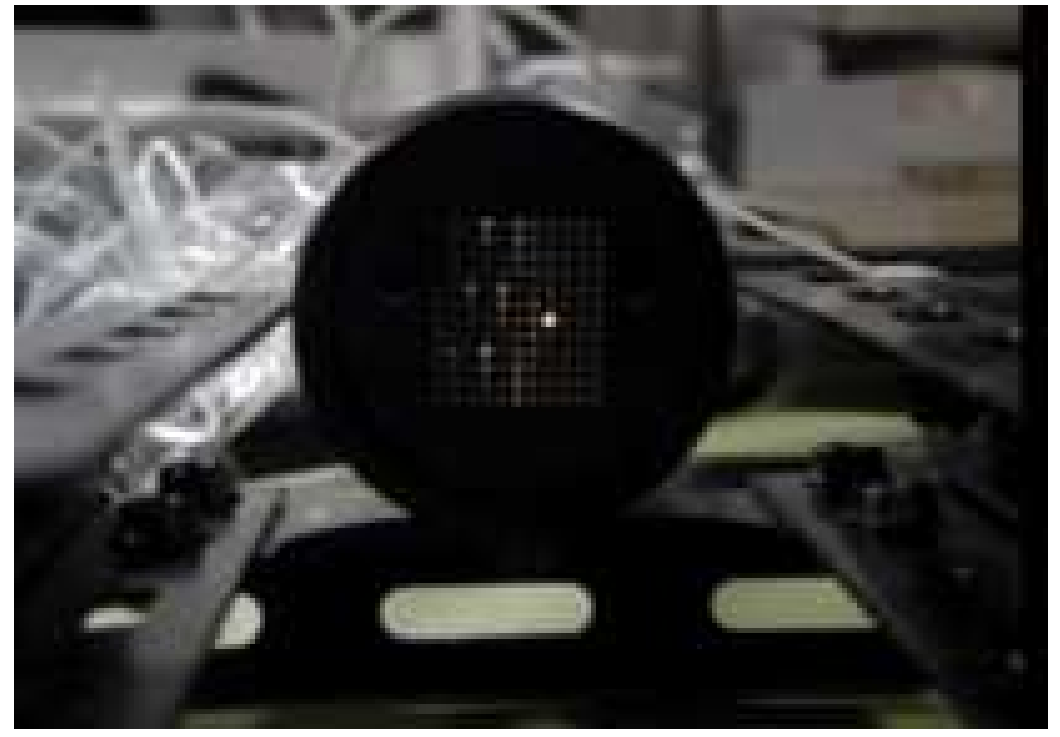

Figure 6.21: Snapshot of the moving image of a bulkhead connector taken for the test of channel assignment. The channel being tested is seen in the middle on the connector.

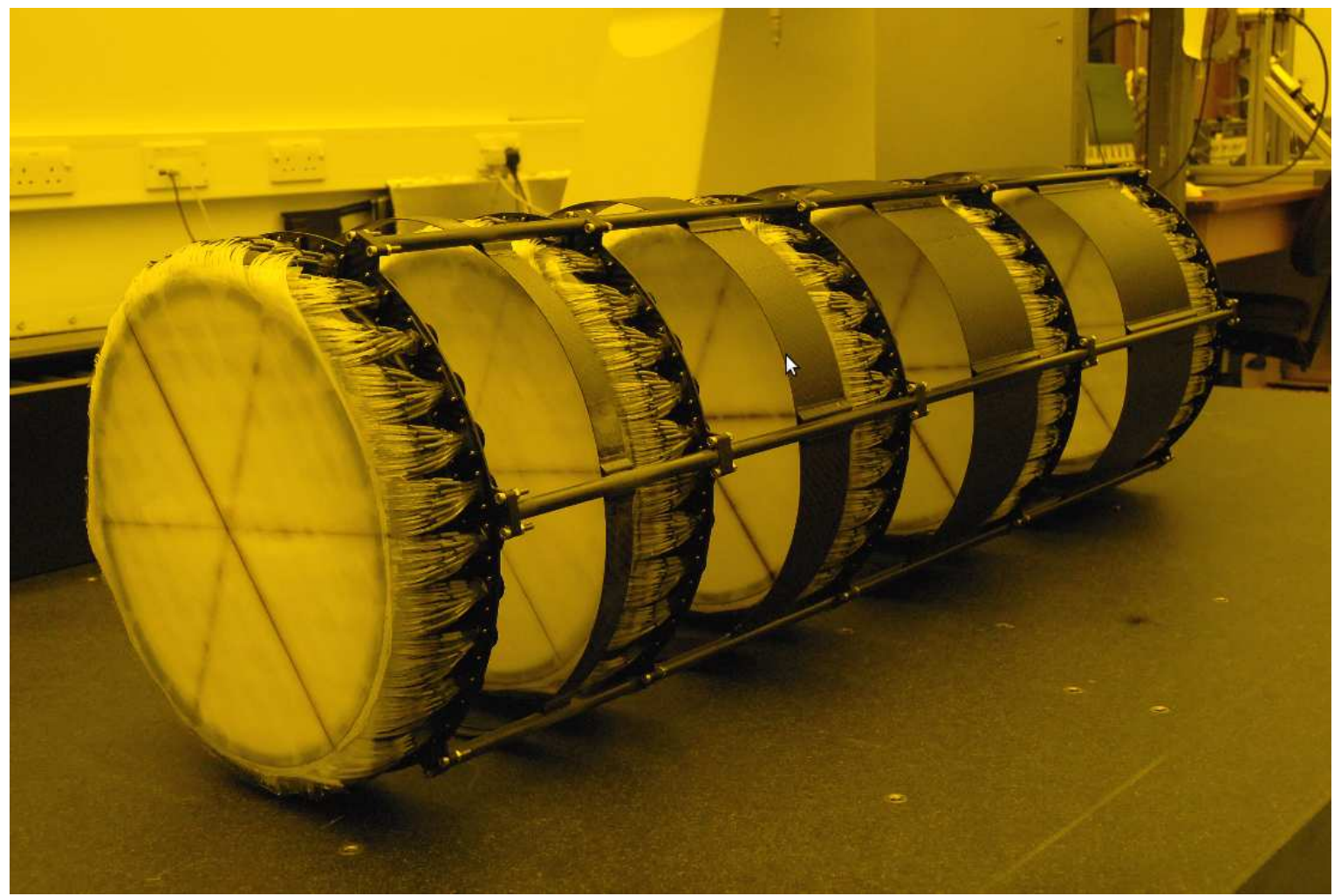

Figure 6.22: Photograph showing a tracker of five stations each are fixed with station support frame. 


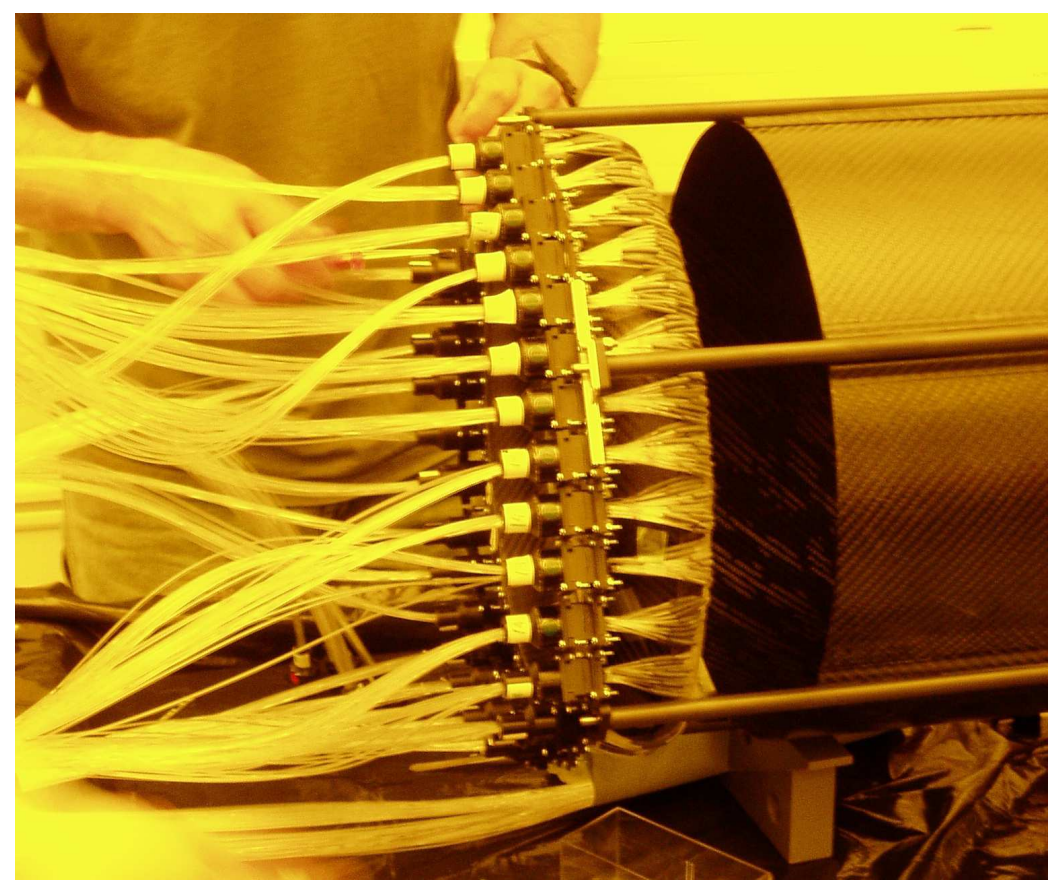

Figure 6.23: Photograph showing internal light-guides are carefully being attached with the Station No.5.

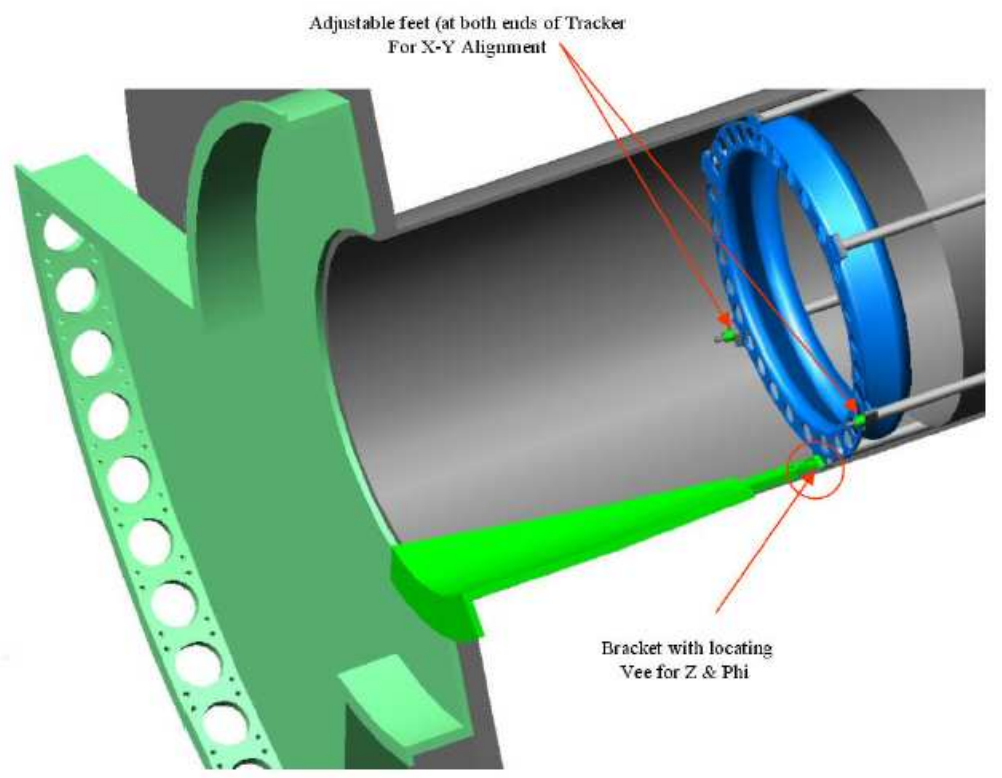

Figure 6.24: Station alignment mechanism. The part shown in green will be dowelled to the spectrometer solenoid end plate. At the narrow end of the part, inside the bore of the solenoid, a locking mechanism will be used to locate the tracker in $\mathrm{z}$ and in azimuth. 


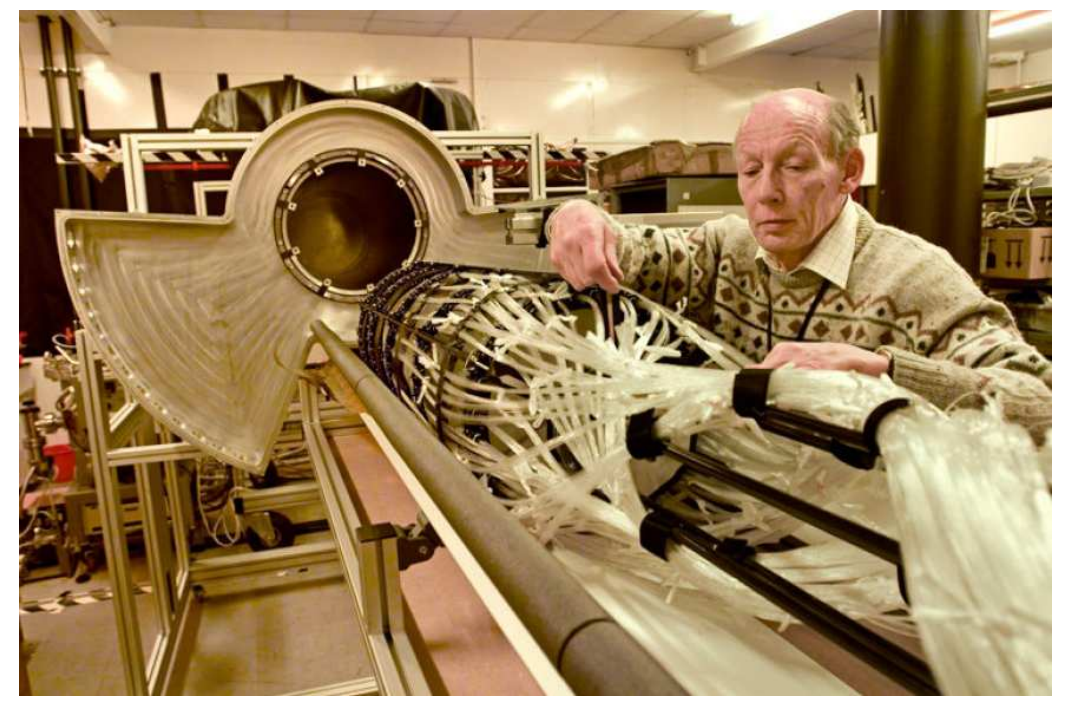

Figure 6.25: Photograph showing that the SciFi tracker with the internal light-guides is being installed into the light-tight storage box for cosmic-ray test.

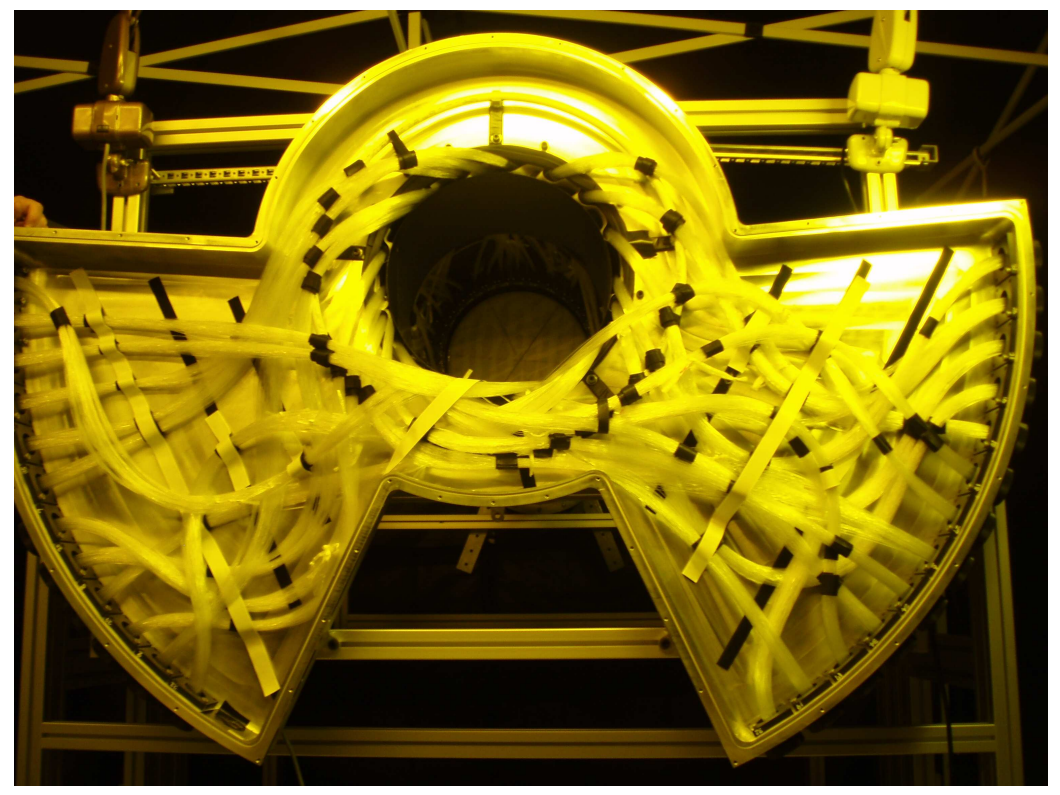

Figure 6.26: Photograph of the internal light-guides supported within the patch panel. The photograph was taken at the end of the assembly of the first tracker in the cosmic test stand. The light-guides are held out of the tracking volume using a sprung carbon-fiber collar inserted into the bore. 


\section{Chapter 7}

\section{Study of Performance of SciFi Trackers}

The SciFi tracker No.1 has been tested with cosmic-rays at the RAL in 2008, and tracker No.2 at the RAL in 2009. At first, a setup of the test bench for cosmic-ray test is explained. Secondary, implementations of softwares for a tracker slow control and DAQ are described. Thirdly, the procedures of analysis for the cosmic-ray data is described, and the performances are presented for Trackers No.1 and No.2 in particular their alignments and efficiencies, which are important for resolutions of momentum measurements, are examined.

\subsection{Setup of cosmic-ray test}

The trackers were placed in the support stand vertically with respect to the ground floor. The patch panel was horizontally attached at the top of the tracker. The cryostats for the VLPC readout (No.1 and No.2 for Tracker No.1 and No.3 and No.4 for Tracker No.2) were located beside the support stand (Figure 7.1). The cryocooler on the cryostat, which was used to cool the two cryostats, was connected with flex tubes (supply/return) with a Sumitomo helium compressor. Water was used to cool the compressors during the operation (Figure 7.3).

External light-guides were attached with optical connectors at the patch panel. The other ends were connected with the optical connectors on the cryostat with optical grease applied (Figure 7.4). The connection assignment of the external light-guides to the optical connectors on cryostat for Tracker No.1 and No.2 are summarized in Figure 7.15.

Plastic scintillating counters prepared by the FNAL were used for trigger counters (Figure 7.5). At both of the top and bottom, two plastic scintillation counters were put together with 90 degrees rotation one another to define a fiducial area of $30 \mathrm{~cm}$ diameter circle of the stations. A distance between the top and bottom counters was approximately $200 \mathrm{~cm}$. Lead blocks were put above the bottom counters in order to select high-energy muons by coincidence of the top and bottom counters.

\subsection{Tracker read-out}

Electronics used for the test was described in Subsection 7.2.1. The trigger logic is described in Subsection 7.2.2. The implementations of the tracker slow control and DAQ are described in 


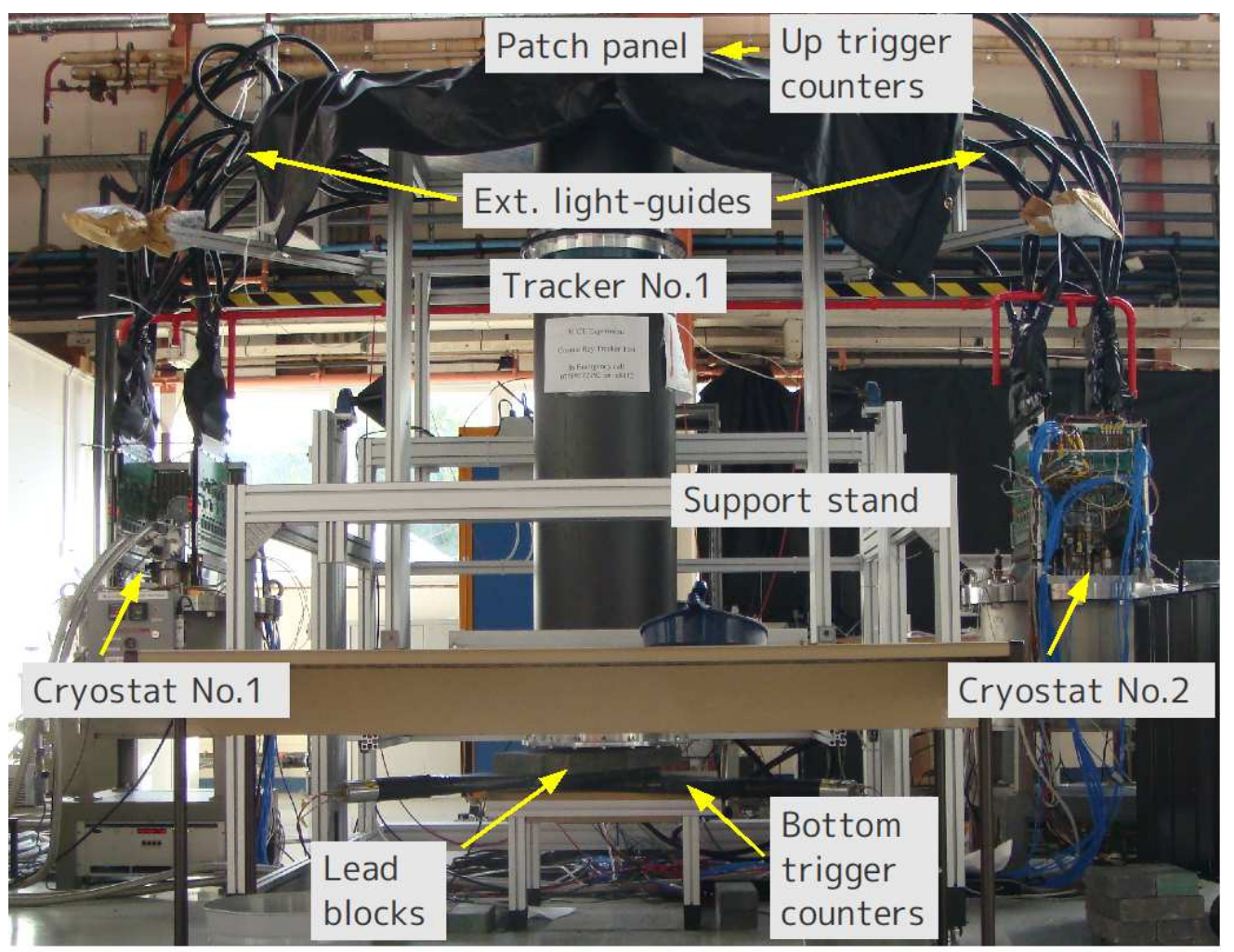

Figure 7.1: Tracker No.1 are in place at R8 experimental hall in the RAL.

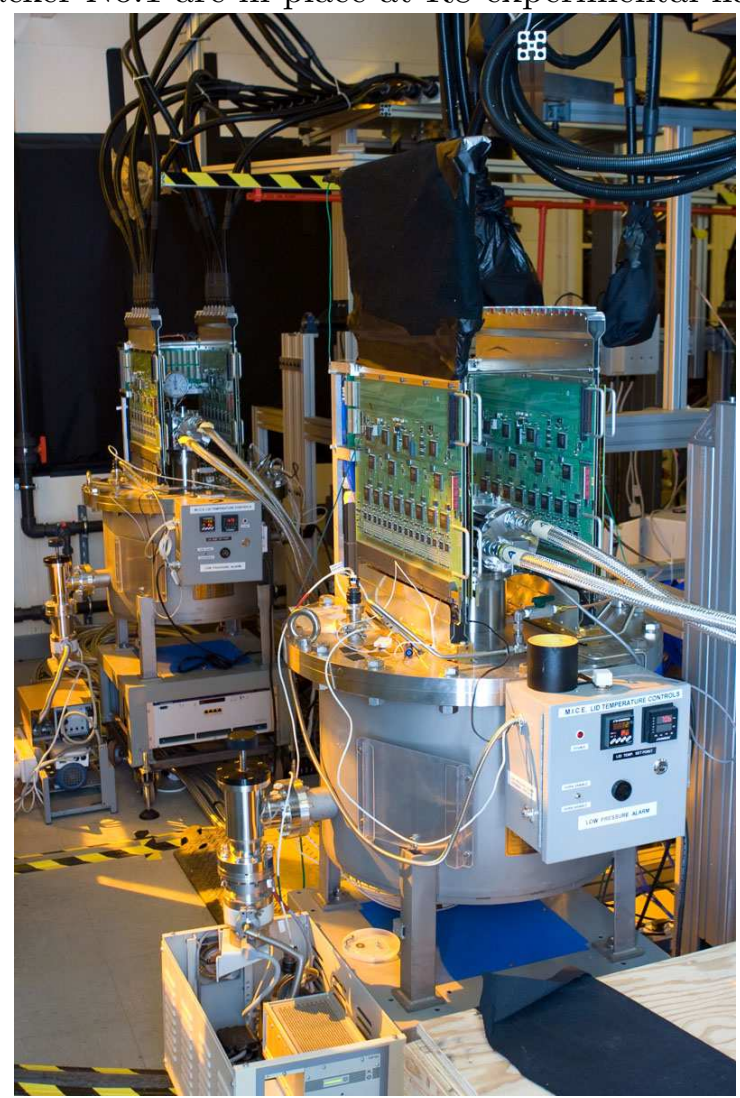

Figure 7.2: Tracker No.2 are in place at Lab7 experimental room in the RAL. 

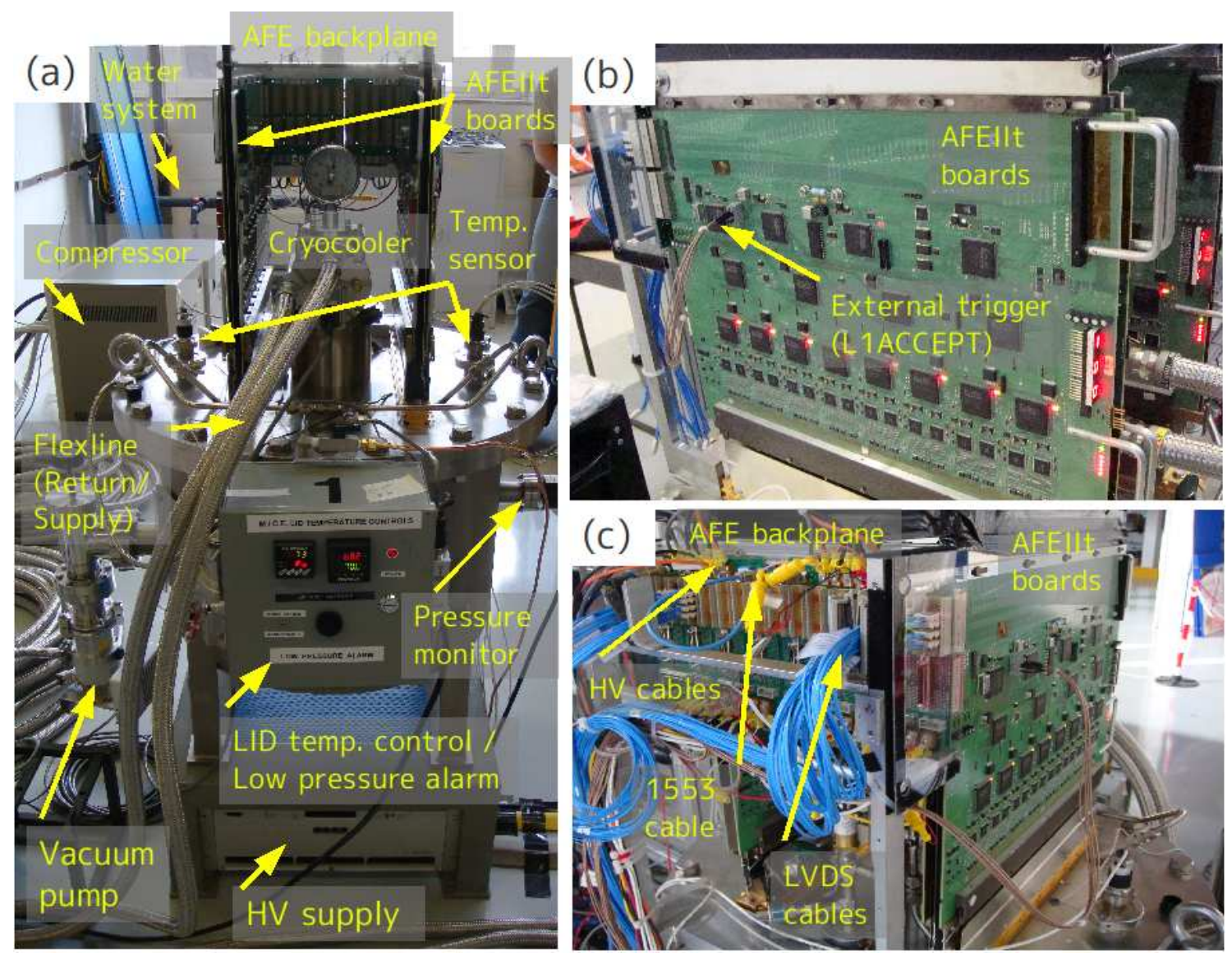

Figure 7.3: (a) Photograph of the cryostat No.1. On the back side of the cryostat, the AFE back plane is attached. Four AFEIIt boards are attached. The HV supply unit is located under the cryostat. In the middle, the cryocooler with two flex tubes (return/supply) is mounted. The flex tubes are connected with the compressor unit located behind the cryostat. The compressors are water-cooled during the operation. The water is being cooled by the chiller (not shown here). Temperature sensors are connected on the lid of the cryostat. The other ends are connected to the Oxford temperature controller in the rack. The pressure monitor is attached on the side of the cryostat. At the other side of the cryostat, the vacuum pump is attached. On the front of the cryostat, the lid temperature and low pressure alarm unit is attached. (b) Photograph of the AFEIIt board mounted on the cryostat. The external trigger (L1ACCEPT) is injected to the board directly to the board. (c) Photograph of the AFE back plane. The HV cables, LVDS cables and MIL-1553 cable are connected with the back plane. 

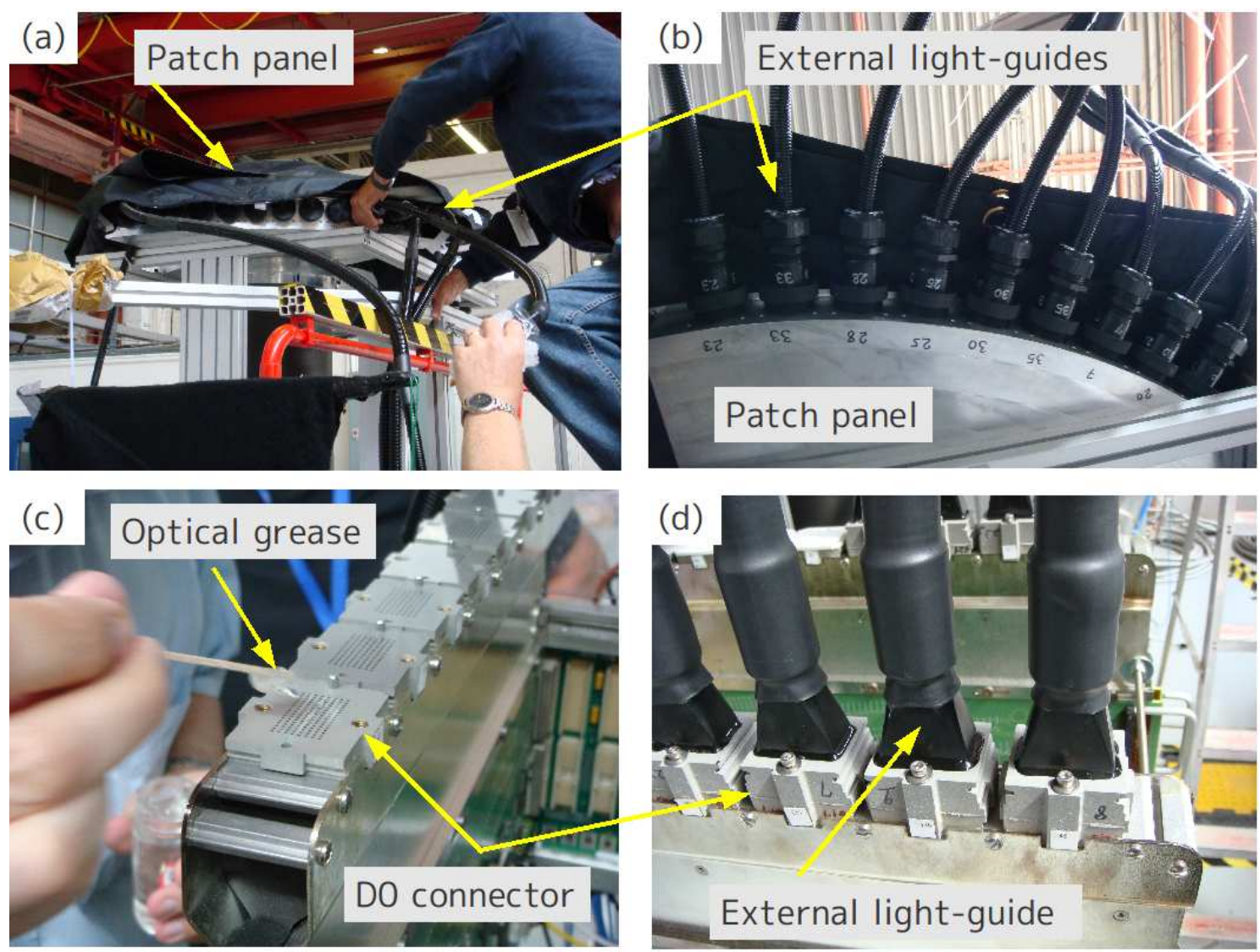

Figure 7.4: Photographs shows the attachment of external light-guides. An external light-guide is being attached with the bulkhead connector with optical grease applied at the path panel by turning the locking ring (a). The patch panel with external light-guides attached, viewed from the cryostat No.2 (b). With optical greases applied on the surface of the D0 connector (c), external light-guides are fixed on the cryostat with two screws (d). 

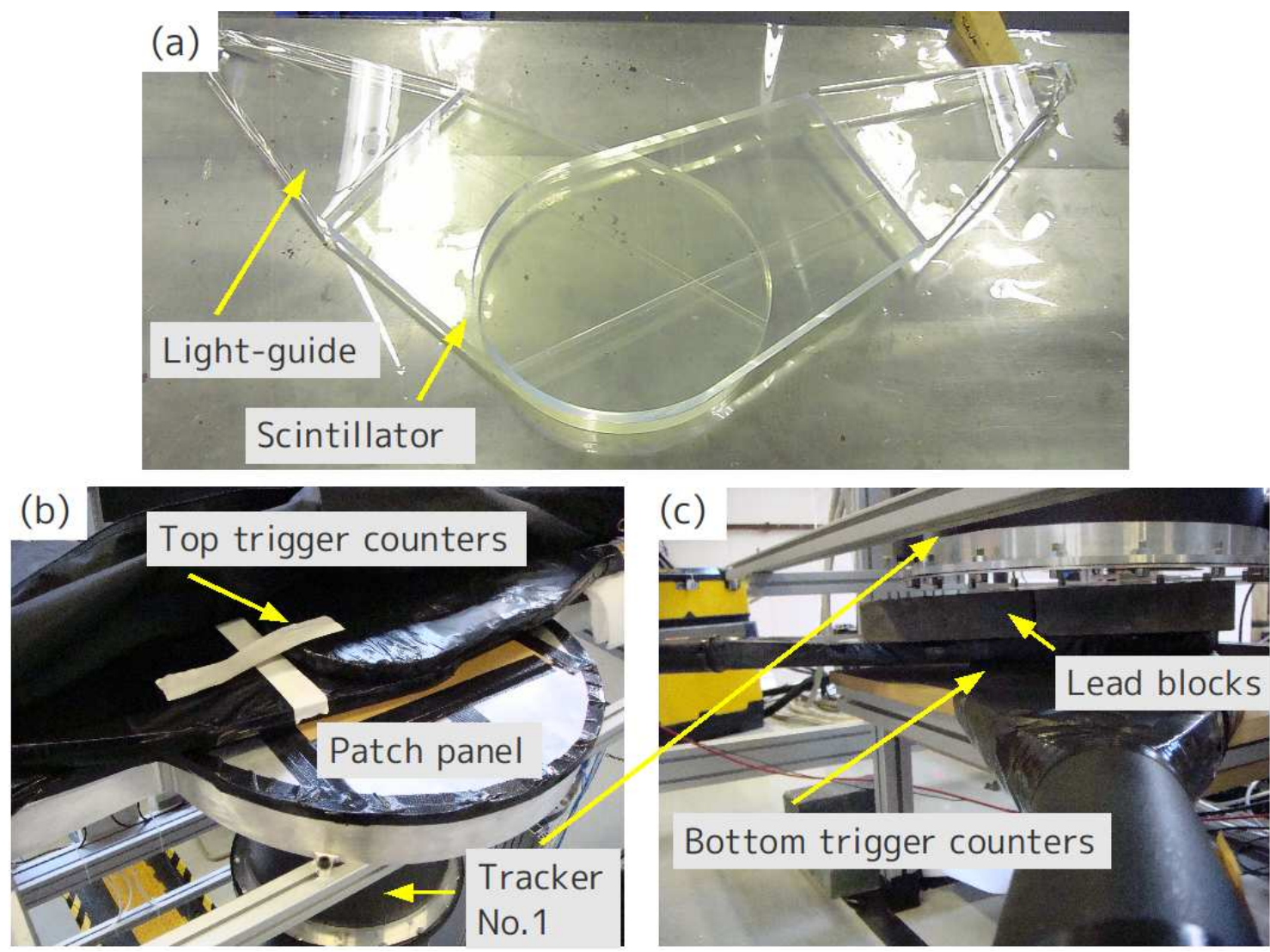

Figure 7.5: (a) Two sets of scintillating counters manufactured at the FNAL. Each are put together with 90 degrees rotation as shown to define a circle of $30 \mathrm{~cm}$. Those will be attached with PMTs and shielded with black-out tape. (b) Trigger counters located on the patch panel. (c) Trigger counters located under the tracker. Lead blocks are put on the counter. 
Subsection 7.2.3 and 7.2.4.

\subsubsection{Electronics}

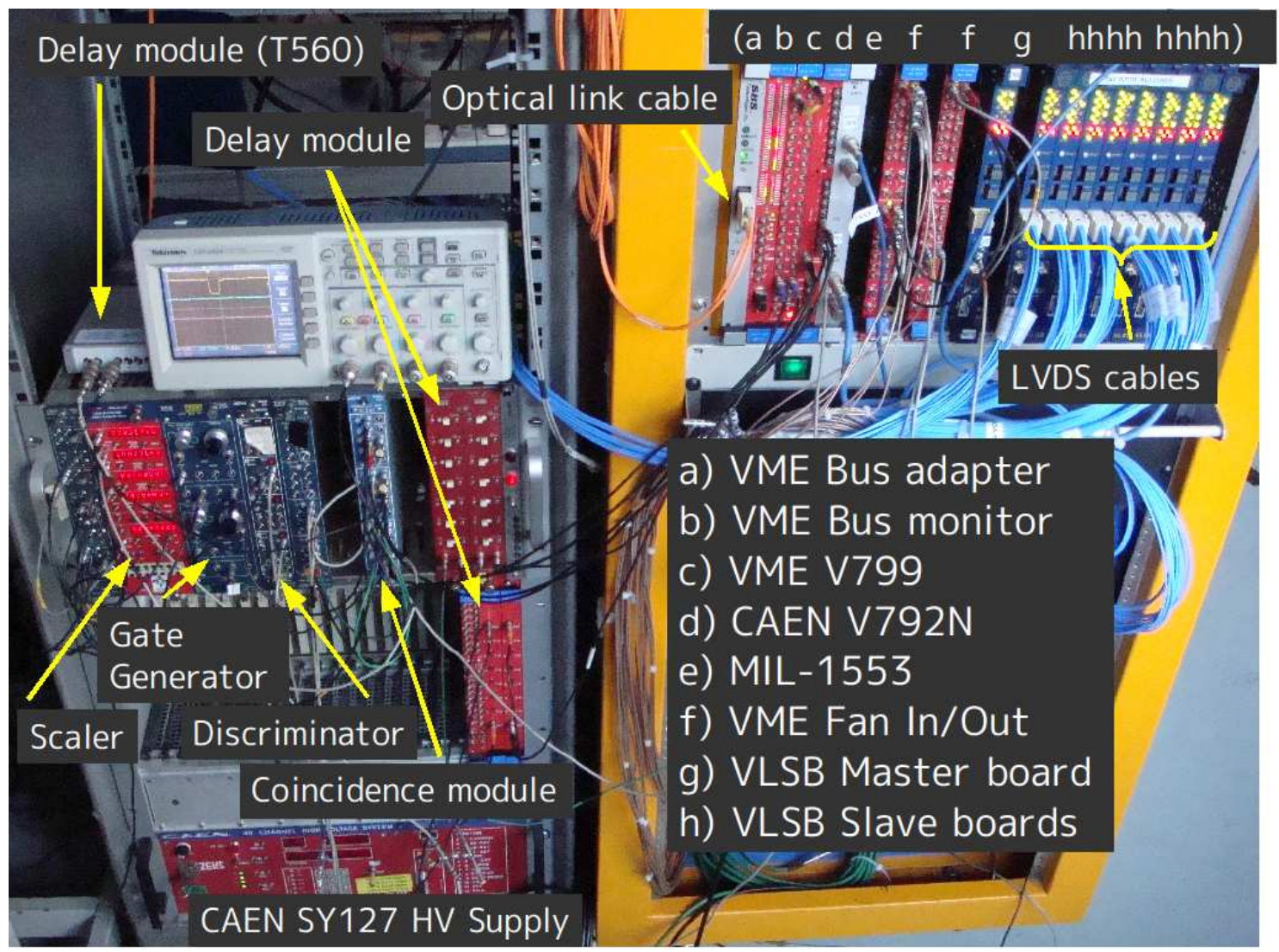

Figure 7.6: Photograph shows the creates used for the test. The left-hand is the NIM crate to make triggers, and right-hand is the VME crate to operate AFEIIt boards and take data. The details on the boards installed in the creates are described in text.

Figure 7.6 shows a photograph of the DAQ crates. The rack at the right hand was a VME crate to operate the AFEIIt boards and take data, while the one at the left hand rack had a NIM crate to make trigger signals.

The boards (modules) used for the test are described as follows. The descriptions of boards related with the tracker DAQ are described in Section 4.3.2.

\section{AFEIIt board}

Eight AFEIIt boards were installed on the VLPC cryostats, each of which were connected via four LVDS cables with the VLSB boards.

\section{VLSB slave board}

Eight VLSB boards were installed in the VME crate to store tracker data sent from the AFEIIt boards. 


\section{VLSB master board}

One VLSB master board was used to send trigger signal to AFEIIt boards. The input (TTL) was the output of the T560 module. The output (TTL) was sent to the VME Fan In/Out board.

\section{MIL-1553 board}

The MIL-1553 board was connected to the back plane on the cryostat via the serial cable. One channel was for the cryostat No.1 and another was for the cryostat No.2.

\section{CAEN V799 board}

The CAEN V799 board (16 Channel Input/Output Register) was used in order to control the trigger signal. The input (NIM) was the output from the coincidence module. The output (NIM) was directed to the VETO channel of the coincidence module.

\section{CAEN V792N board}

The CAEN V792N board (16 Channel Multievent QDC) was used in order to measure the energy deposit in trigger counters. The input to the gate channel (NIM) was the output from the gate generator module. The width of the input was set to 100 nsec. The anode signals from PMTs were connected with the channels (from No.1 to No.4) via LEMO-cables.

\section{T560 delay module}

The HIGHLAND T560 delay module was used to adjust the timing of the trigger. The programmable delay was set via the Ethernet.

\subsubsection{Trigger logic}

The diagram of the trigger logic is shown in Figure 7.7.

The four outputs of PMTs were discriminated and forwarded to the coincidence module, where signals were 'and'ed. The output of the coincidence module (NIM) was forwarded to the CAEN V977 board. One of the output of the CAEN V977 board (NIM) was sent back to the coincidence module (VETO), which was generated by the hardware soon after the trigger signal was sent to the input. Another output (NIM) was converted from NIM to TTL at a NIM module then sent to the HIGHLAND T560 delay module. The output of the delay module T560 (TTL) was injected to the VLSB master board. The output of the VLSB master board (TTL) was divided by the VME Fan In/Out modules then sent to the AFEIIt boards (L1ACCEPT).

The trigger was repeatedly checked if it had been arrived at the CAEN V977 board and been accepted at the VLSB master board in the DAQ (busy wait). The details are described in the VLSB master board in Section 4.3.2.3.

\subsubsection{Timing adjustment}

There was a need to adjust the timing of the trigger signal sent to the AFEIIt boards. As noted in Section 4.3.2, the Trip-t chip on the AFEIIt board sampled the output electric charges from VLPCs and stored them into buffers (made of capacitor arrays) in the pipeline. Upon reception of external trigger signals (L1ACCEPT), the Tipt-t started to digitize the electric charges stored in

the buffer, of which location is determined by the timing of L1ACCEPT. By adjusting the timing of L1ACCEPT to be at off time, the signal-stored buffer can be obtained. 


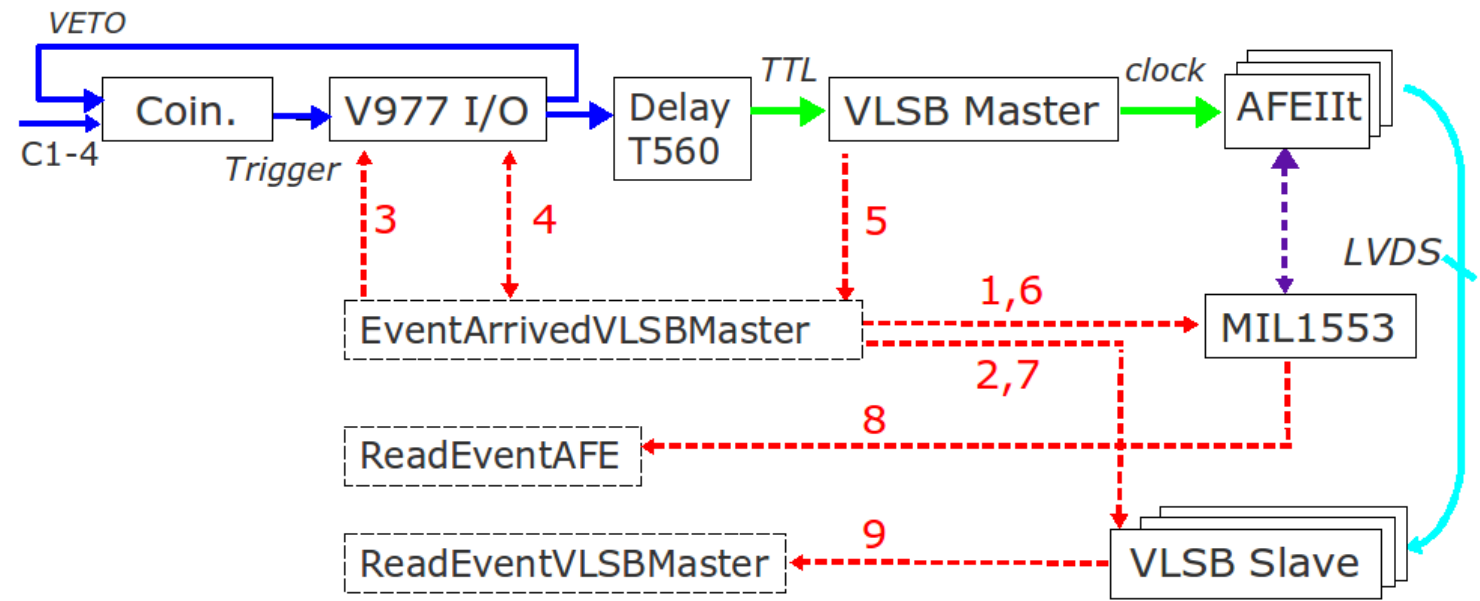

Figure 7.7: Diagram of the trigger logic for the cosmic-ray test. The solid arrow indicates the signal flow by the board (NIM(blue), TTL(green), LVDS(light-blue)), while the dashed arrow indicates the command flow by the DAQ. The solid square means the name of the board (module), while the dashed square means the name of the function in the DAQ. The number beside the arrow corresponds to the order of the access. The actual operation in each command is described in the VLSB master board in the section xxx.

The strategy of the timing adjustment was as follows (Figure 7.8). First, the correct timing was investigated using LED lights. The tuning parameters were set at the VLSB master board via the VME access. Next, the time difference expected at the setup of the cosmic-ray measurements was estimated as follows. The time (T2-T1) and (T3-T1) at the LED measurements corresponded to the time (T6-T5) and (T3-T5) at the cosmic-ray test, respectively. The timing difference was therefore calibrated by setting the delay by the time ((T3-T5)-(T3-T1))-((T6-T5)-(T2-T1)).

To make sure that the adjustment was made correctly, cosmic-ray data were taken with several different timings around the calculated timing by changing additional delay of timing at the T560 delay module. The measured light-yield as a function of additional delay timing is shown in Figure 7.9. From this, the delay was set to the $862 \mathrm{~ns}$ where the maximum light-yields were obtained.

\subsubsection{Tracker slow control}

Figure 7.10 describes the process variables implemented for the AFEIIt board. When AFEIIt clients write the value 1 to CONFIG-XXX PVs (XXX indicates the target to be initialized), the AFEIIt server does access to the AFEIIt board to perform the initialization. If it succeeds, those PVs are changed to 1 . If it fails, the error is raised to the clients and the process is terminated. If ALL is specified, all of the boards are configured.

When the clients read the CONFIG-XXX PVs, the AFEIIt server just returns the corresponding PVs to the clients. The value 1 means "configured". Prior to the data-taking, the tracker DAQ must read this PV to check whether all of the boards have been configured.

The trigger for the AFEIIt board is enabled (disabled) by writing 1 (0) to the PV, TRIGENABLE. The trigger mode is needed to be changed by the DAQ every after the trigger is received to the AFEIIt boards. The procedure of changing the trigger mode is described in the next subsection. 


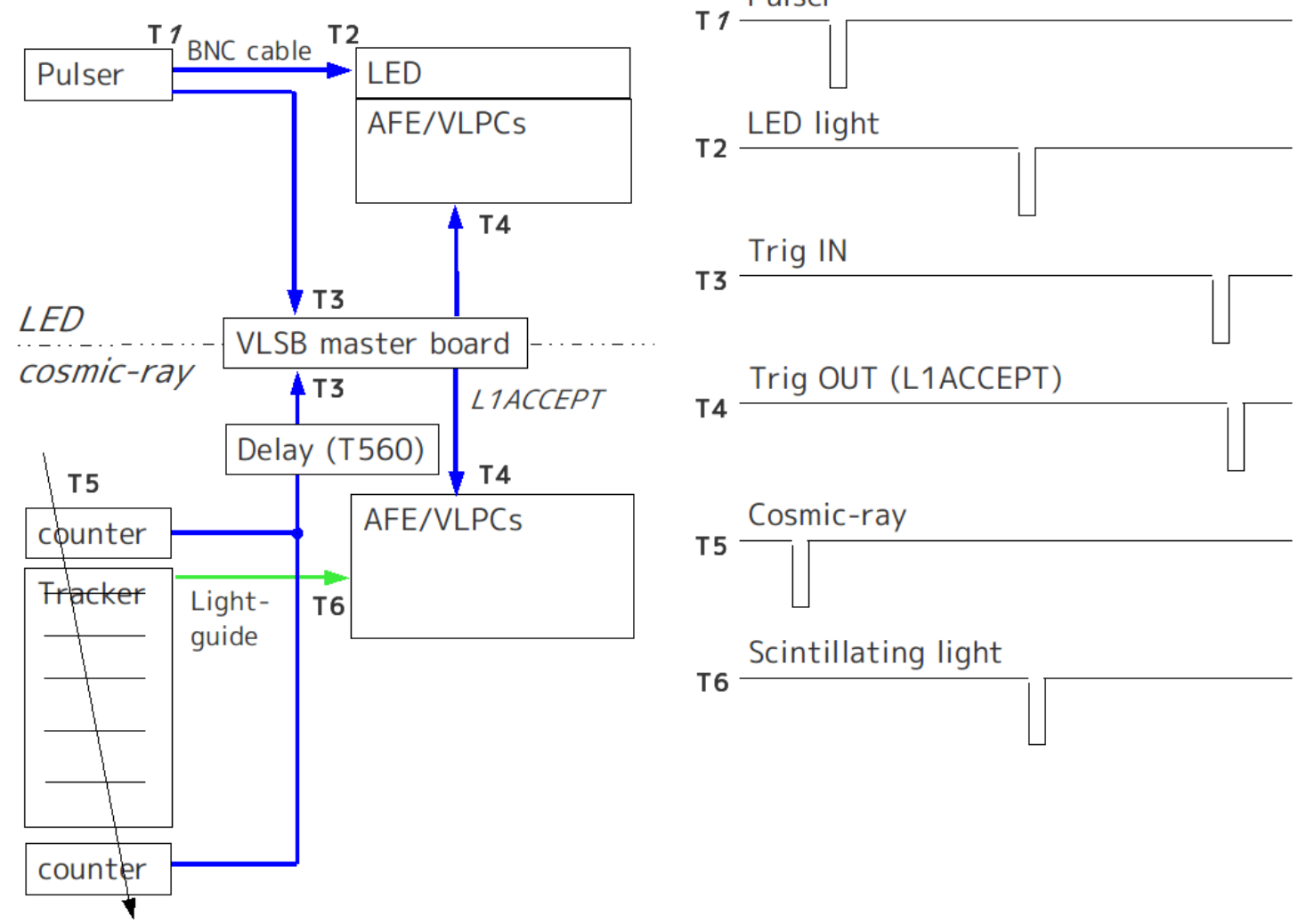

Figure 7.8: Setup (left) and timing chart (right) for the trigger timing adjustment. Blue lines indicates the BNC (LEMO) cables and green lines indicates the light-guide (external+internal). In the setup of the LED measurement, the trigger from the pulser is transfered to the VLSB master board. The L1Accept signal is then transfered to the AFEIIt board. In the setup of the cosmic-ray test, the coincidence signal is transfered to the delay module T560 before the VLSB master board to adjust the delay. 


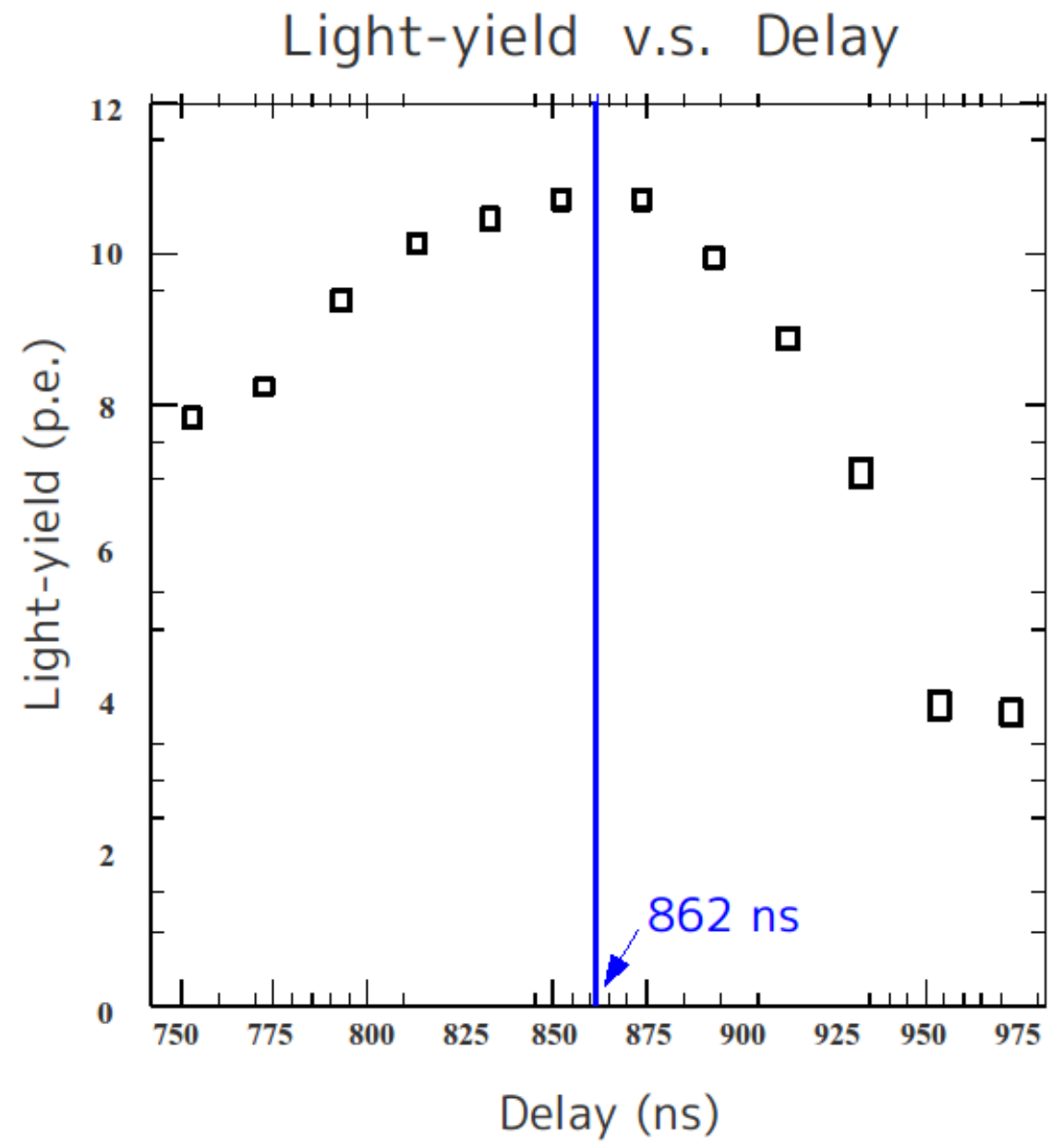

Figure 7.9: Light-yield as a function of the delay. The maximum light-yield is expected at $862 \mathrm{~ns}$ delay. 


\begin{tabular}{|c|c|c|c|c|}
\hline Cassette PV Type & Data Type & Size & Write Functionality & Read Functionality \\
\hline CONFIG-ALL & aitInt8 & 1 & $\begin{array}{l}\text { Write "1": all current description } \\
\text { parameters written to Cassette }\end{array}$ & $\begin{array}{l}\text { Get configuration state of all } \\
\text { Cassette subsystems ( } 1= \\
\text { configured) }\end{array}$ \\
\hline CONFIG-BOARD & aitInt8 & 1 & $\begin{array}{l}\text { Write " } 1 \text { ": current Board } \\
\text { Description parameters written to } \\
\text { Cassette }\end{array}$ & $\begin{array}{l}\text { Get Cassette Board configuration } \\
\text { state }(1=\text { configured })\end{array}$ \\
\hline CONFIG-TRIPTS & aitInt8 & 1 & $\begin{array}{l}\text { Write "1": current TriPt } \\
\text { Description parameters written to } \\
\text { Cassette }\end{array}$ & $\begin{array}{l}\text { Get Cassette TriPt configuration } \\
\text { state }(1=\text { configured })\end{array}$ \\
\hline CONFIG-BIAS & aitInt8 & 1 & $\begin{array}{l}\text { Write " } 1 \text { ": current Bias } \\
\text { Description parameters written to } \\
\text { Cassette }\end{array}$ & $\begin{array}{l}\text { Get Cassette Bias configuration } \\
\text { state }(1=\text { configured })\end{array}$ \\
\hline CONFIG-CRYO & aitInt8 & 1 & $\begin{array}{l}\text { Write " } 1 \text { ": current Cryo } \\
\text { Description parameters written to } \\
\text { Cassette }\end{array}$ & $\begin{array}{l}\text { Get Cassette Cryo configuration } \\
\text { state }(1=\text { configured })\end{array}$ \\
\hline TRIG-ENABLE & aitInt8 & 1 & $\begin{array}{l}\text { Write " } 1 \text { ": enable triggers } \\
\text { Write "0": disable triggers }\end{array}$ & $\begin{array}{l}\text { Get trigger enable state }(1= \\
\text { enabled) }\end{array}$ \\
\hline TEMP & aitFloat64 & 8 & N/A (read only) & Get MCM temperatures \\
\hline HEATER & aitUint16 & 8 & N/A (read only) & Get MCM heater values \\
\hline CRYO-DESC & aitFixedString & 500 & Set Cryo Description XML string & Get Cryo Description XML string \\
\hline $\begin{array}{l}\text { STATUS-READ- } \\
\text { ENABLE }\end{array}$ & aitInt8 & 1 & $\begin{array}{l}\text { Write " } 1 \text { ": enable status } \\
\text { monitoring } \\
\text { Write " } 0 \text { ": disable status } \\
\text { monitoring }\end{array}$ & $\begin{array}{l}\text { Get status monitoring enable state } \\
(1=\text { enabled) }\end{array}$ \\
\hline $\begin{array}{l}\text { STATUS-READ- } \\
\text { ENABLED }\end{array}$ & aitInt8 & 1 & N/A (read only) & $\begin{array}{l}\text { Get status monitoring enable state } \\
(1=\text { enabled })\end{array}$ \\
\hline
\end{tabular}

Figure 7.10: List of process variables (PVs) for the tracker slow control. 
By reading the PV, TEMP (HEATER), the AFEIIt clients know the current temperatures (heater values) of the cassettes. At each time when the clients read this PV, the server accesses to the AFEIIt board to get the value. In the cosmic-ray test, cassette temperatures and heater values were read out by the DAQ and recorded in the DATE events to test this functionality. Usually, as at the MICE physics runs, these cassette information are read out by the monitoring process (not by DAQ) once after the cryostats are cooled down to monitor that temperatures are stable at $9 \mathrm{~K}$.

\subsubsection{Tracker DAQ}

The equipments of the tracker DAQ implemented for the cosmic-ray test with their parameters defined in the database are described in Figure 7.11. When the 'start process' starts, the Arm functions of these equipments are called to initialize their boards. When the DAQ starts, the process stops at the EventArrived function, where the trigger is waited (busy wait). When the trigger is accepted, the Read functions of the CAEN V792N board, the AFEIIt and VLSB slave board are called in sequence to read the data. This process is repeated until the number of triggers reaches at the maximum number defined in the database, and then the DisArm functions of these equipments are issued.

The functionalities of these equipments are as follows. The VME bus adaptor is an interface board between the PCI bus and the VME bus. The AFE is the AFEIIt CA client for EPICS to manage its initialization, the trigger mode and monitoring of the cassettes. The CAEN V799 board controls the VETO signal and checks whether the trigger is arrived. The CAEN V792N baord records ADCs which correspond to deposit energies in the trigger counters. The VLSB master board generates the trigger signal (L1ACCEPT) which is sent to the AFIIt boards and controls the VLSB slave boards. The VLSB slave boards store tracker data transfered from the AFEIIt boards into their memory banks.

The followings describes their functions in detail.

\section{VME bus adaptor}

Open (close) the device file in the Arm (DisArm) function.

\section{CAEN V799 board}

The board initializes the board in the Arm function. This is handled by the VLSB master board in its EventArrived function to clear the output register.

\section{CAEN V792N board}

The board initializes the board in the Arm function. In the Read function, ADCs at 16 channels (4 channels are connected to the PMTs) are read out, then the board is reset for the next trigger.

\section{AFEIIt board}

The board requests the status of the initialization to the AFEIIt CA server in the Arm function. If the AFEIIt boards are not initialized, the DAQ terminates with an error informing the message. In the current implementation, the initialization at the first time is performed independently from the DAQ.

In Read function, ask to the AFEIIt CA server to get the temperature and heater values of the cassettes. In future MICE physics runs, this function will be disabled.

This board is also handled by the VLSB master board in its EventArrived function to set the 


\section{List of equipments}

\begin{tabular}{|l|l|l|l|l|l|}
\hline Name & Description & ID & D & T & $\# /$ pars \\
\hline VME & VME bus adapter & 40 & 0 & 0 & 0 \\
\hline AFE & AFEIIt boards & 50 & 1 & 0 & 1 \\
\hline V977 & CAEN I/O Register & 60 & 0 & 1 & 2 \\
\hline V792N & CAEN QDC (16ch-lemo) & 70 & 1 & 0 & 2 \\
\hline VLSBSlave & VLSB board & 80 & 1 & 0 & 6 \\
\hline VLSBMaster & $\begin{array}{l}\text { VLSB trigger board/ } \\
\text { VLSBSlave controller }\end{array}$ & 90 & 0 & 1 & 10 \\
\hline
\end{tabular}

\section{List of parameters}

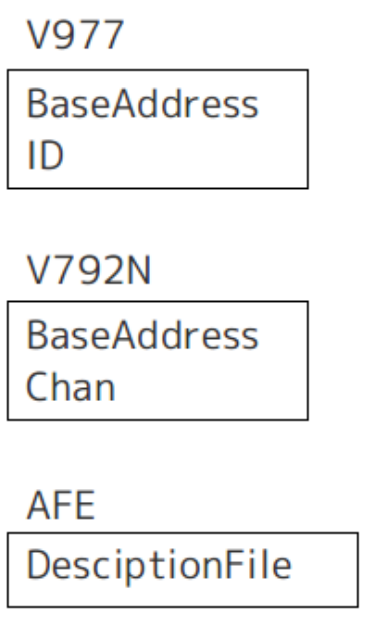

VLSB Master
\begin{tabular}{|l|}
\hline BaseAddress \\
Spill_duration \\
Trigger_period \\
MaxEvents \\
Veto_gate_delay \\
Veto_gate_period \\
Veto_window \\
Fast_clear \\
Cont_spill \\
Trig_mode \\
Trig_location \\
\hline
\end{tabular}

VLSB Slave

\begin{tabular}{|l|}
\hline ID \\
AFENum \\
CASSETTE \\
RT \\
BaseAddress \\
BaseAddressOf1553 \\
\hline
\end{tabular}

Figure 7.11: List of equipments (above) and their parameters (bottom) implemented for the cosmicray test. The fourth column (ID) in the above indicates the unique number defined in each equipment. The Arm (DisArm) functions are called in sequence according to this number in ascending (descending) order. The fifth column (D) in the above indicates that its equipment generates data or not (If so, its ReadEvent function is called). In the cosmic-ray test, three boards (AFE, V792N and VLSB) have data to be recorded into the DATE events. The sixth column (T) in the above indicates that its equipment generates trigger or not (If so, its EventArrived function is called). The seventh column in the above shows the number of parameter defined in the database. 
trigger disabled.

\section{VLSB master board}

In the Arm function, the VLSB master board are initialize using their parameters defined in the database. Then, it sets the V977 board be BUSY (VETO) to prevent the next trigger being injected until the AFEIIt and VLSB boards are ready. The AFEIIt and VLSB slave boards are set the trigger disabled.

In the EventArrived function, the AFEIIt and VLSB slave boards are set the trigger enabled. Then, the trigger is waited in the "while loop' as below (busy wait). (1) Clear the VETO at the V977 board by reseting the output register. (2) Check the trigger at the V977 board by reading the input register. (3) Once the trigger is arrived at the V977 board, read the VLSB master board to check whether the trigger is accepted by reading the relevant register. If the trigger is accepted at the VLSB master board, then break 'while loop', otherwise repeat 'while loop'. Finally, the AFEIIt and VLSB slave boards are set the trigger disabled before returning the function.

In the DisArm function, both the AFEIIt and VLSB slave boards are set the trigger disabled.

\section{VLSB slave board}

In the Arm function, the VLSB slave board is initialized and the trigger is disabled. In the Read function, the VLSB buffers are read out, then cleared. The data format is shown in Figure 7.12.

This board is also handled by the VLSB master board in its EventArrived function to set the trigger disabled.

\subsection{Data taking}

Data taking for Tracker No.1 have been started from July 7th 2008 until October 19th 2008 at the R8 in the RAL. Data taking for Tracker No.2 have been started from May 26th 2009 until July 2nd 2009 at the Lab7 in the RAL. The trigger rate (coincidence of trigger counters and L1Accept trigger signal) was about $0.03 \mathrm{~Hz}$. The total number of triggers was 15,323 for Tracker No.1 and 4,049 for Tracker No.2.

DATE DAQ run at the last of the test of Tracker No.1 in order to test the DAQ system itself. Most of data were taken by EXCEL based DAQ. The EXCEL DAQ is the standalone readout system for tracker and not suitable for fast readout, but still good system at tests of a slow trigger rate such as cosmic-ray test.

\subsection{Data analysis}

This section describes a procedure to extract $\mathrm{ADC}$ values from raw data and to calculate hit positions on stations using ADC values.

\subsubsection{Decoding}

The EXCEL DAQ records data in a plain text file. The data format is described in Figure 7.13. By decoding data blocks in four memory banks, ADC values of 512 VLPC channels in a AFEIIt-bard are extracted. In the cosmic-ray test, eight AFEII-t boards are used, so that total channels are 4,096. TDC values were meaningless (not yet tested) in this test and not used for the analysis. 
V792N

\begin{tabular}{|l|l|l|}
\hline name & \#/words & description \\
\hline data & 16 & V792N data $($ ch0 - ch15) \\
\hline
\end{tabular}

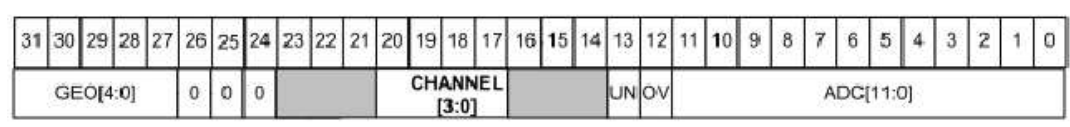

V792N data

\begin{tabular}{|l|l|l|}
\multicolumn{2}{l}{ AFE } \\
\hline name & $\# /$ words & description \\
\hline cassetteSize & 1 & \#/cassettes (currently 4) \\
\hline tempSize & 1 & \#/temperatures (should be 8 ) \\
\hline temperature & tempSize & Temperature (9.0 etc.) \\
\hline heaterSize & 1 & \#/heater values (should be 8) \\
\hline heatervalue & heatSize & Heater value (100 etc.) \\
\hline \multicolumn{3}{|l}{ continue to the next cassette } \\
\hline
\end{tabular}

$\underline{\text { VLSB }}$

\begin{tabular}{|l|l|l|}
\hline name & \#/words & description \\
\hline trigSize & 1 & \#/triggers (currently, 1) \\
\hline bank1Size & 1 & \#/data (180, etc..) \\
\hline bank1data & bank1Size & VLSB 32bit data \\
\hline bank2Size & 1 & \\
\hline bank2data & bank2Size & \\
\hline bank3Size & 1 & \\
\hline bank3data & bank3Size & \\
\hline bank4Size & 1 & \\
\hline bank4data & bank4Size & \\
\hline
\end{tabular}

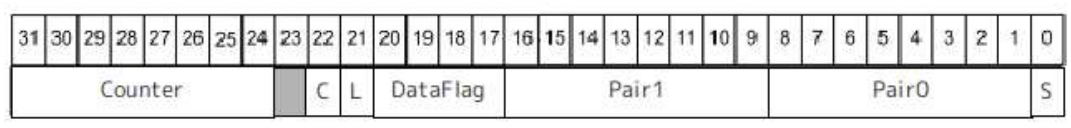

VLSB data

Figure 7.12: (Top) Event structure of the V792N. Sixteen of V792N data (32 bits) are followed in series. (Middle) Event structure of the AFE. Following the number of cassettes, the data blocks of the cassette are followed in series. The format of the data block is shown. (Bottom) Event structure of the VLSB. Following the number of triggers, the data blocks of the trigger data are followed in series. The format of the trigger data is shown. 


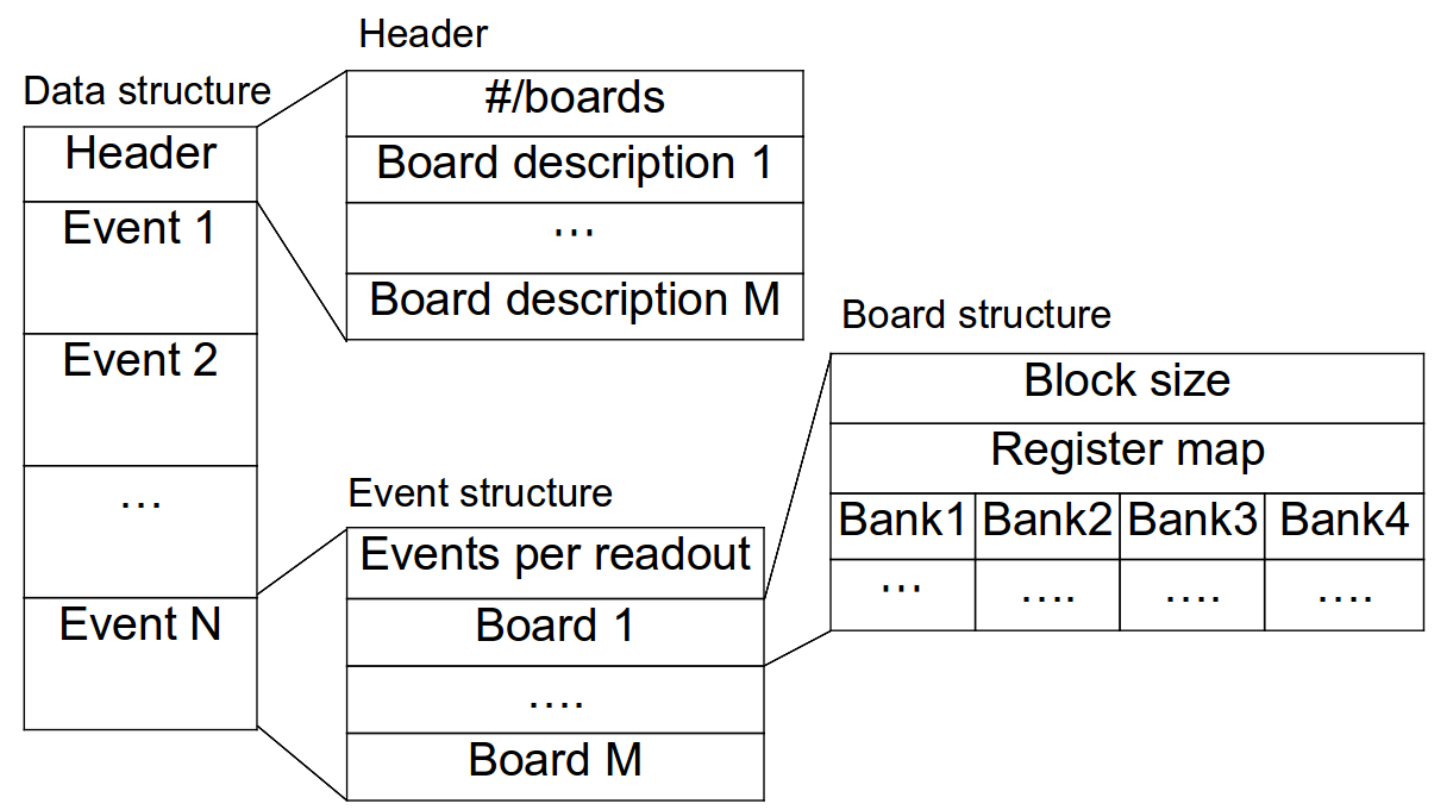

Figure 7.13: Data structure written by the Excel based DAQ in text file. Data is composed of a header and event structures. In the header, number of boards ( 8 in this test) and board descriptions as many as number of boards are written. In a board description, AFEIIt board id, VLPC cassette number, remote terminal (location of slot in back plane) and VLSB board id are written. In a event structure, events per readout (always 1) and board structures are written. In a board structure, block size (always -1), register map (as well as board id and bank data size) and VLSB bank data are written. The structure of VLSB bank data is the same one described in Figure. 


\subsubsection{Calculating light-yield}

In order to remove common mode noise on Trip-chips (16 channels), average of raw ADC values of 12 channels (except for two high and two low ADCs' channels) are subtracted from 16 channels. The pedestal was then estimated by the mean of ADC values subtracted by common mode. Dividing the pedestal subtracted ADC values by the gains (ADC counts per one photo-electron), the light-yields are estimated. VLPC gains are determined by LED data as described in Section xxx.

\subsubsection{Converting VLPC channel number to fiber number}

VLPC channels are converted to fiber number on stations using following mapping information.

(1) VLPC channel to fiber number on D0 connector First step is to convert VLPC channel numbers into fiber number of D0 connector and connector's number. 64 VLPC channels on left-hand AFEIIt board and 64 VLPC channels on right-hand AFEIIt board are connected with one D0 optical connectors via light-guides. Figure 7.14 shows this connection.

(2) D0 connector to external light-guide number Second step is to convert D0 connector numbers into external light-guide numbers. There are eight D0 connectors in each cassettes and there are two cassettes in a cryostat. Table 7.15 shows the connections for Tracker No.1 and Tracker No.2.

(3) External light-guide number to internal light-guide number Third step is to convert external light-guide number to internal light-guide number. External light-guides are attached with internal light-guides at the patch panel. In both trackers, external light-guides were attached with internal light-guides of the same number.

(4) Internal light-guide connector to Station connector Fourth step is to convert connector numbers of internal light-guides into Station connector numbers. Five internal light-guides are attached with one station in defined locations as shown in Figure 6.1. Table 7.16 shows the connections for Tracker No.1 and Tracker No.2.

(5) Station connector to fiber number in view Last step is to convert fiber numbers of internal lightguides into fiber numbers of views. The fiber numbers of views are assigned in anticlockwise rotation from $\mathrm{C} 1$ to $\mathrm{C} 30$ station connector in Figure 6.1.

\subsubsection{Finding hits}

Hits are defined as fibers whose light-yields (with clustering applied) are greater than 2.5 photoelectors in this analysis.

Clustering is applied by adding the light-yield on the adjacent channel, which is effective when a charged particle passes through two fibers, each are connected with adjacent channels, and each deposit energies are both below the threshold.

\subsubsection{Finding triplets}

Triplets hit positions are determined by the hit fibers on the three views which are geometrically overlapped. The hit positions of triplets $\left(x_{t r i}, y_{t r i}\right)$ are defined by;

$$
x_{\text {tri }}=\left(x_{v}+x_{w}\right) / 2, \text { and }
$$




\begin{tabular}{|c|c|c|c|c|c|c|c|}
\hline 30 & 29 & 31 & 32 & 34 & 33 & 35 & 36 \\
\hline 27 & 28 & 26 & 25 & 41 & 38 & 37 & 39 \\
\hline 22 & 21 & 23 & 24 & 40 & 43 & 44 & 42 \\
\hline 19 & 20 & 18 & 17 & 47 & 48 & 46 & 45 \\
\hline 14 & 13 & 15 & 16 & 50 & 49 & 51 & 52 \\
\hline 11 & 12 & 10 & 9 & 57 & 54 & 53 & 55 \\
\hline 6 & 5 & 7 & 8 & 56 & 59 & 60 & 58 \\
\hline 3 & 4 & 2 & 1 & 64 & 63 & 62 & 61 \\
\hline 61 & 62 & 63 & 64 & 1 & 2 & 4 & 3 \\
\hline 58 & 60 & 59 & 56 & 8 & 7 & 5 & 6 \\
\hline 55 & 53 & 54 & 57 & 9 & 10 & 12 & 11 \\
\hline 52 & 51 & 49 & 50 & 16 & 15 & 13 & 14 \\
\hline 45 & 46 & 48 & 47 & 17 & 18 & 20 & 19 \\
\hline 42 & 44 & 43 & 40 & 24 & 23 & 21 & 22 \\
\hline 39 & 37 & 38 & 41 & 25 & 26 & 28 & 27 \\
\hline 36 & 35 & 33 & 34 & 32 & 31 & 29 & 30 \\
\hline
\end{tabular}

Figure 7.14: Arrangement of VLPC channels on D0 connector viewed from surface of an external light-guide. 64 (4x16) in the left square box are read out by left-hand board (RHB) and 64 channels in the right square box are read out by left-hand board (LHB).

Tracker No.1

\begin{tabular}{|c|c|c|}
\hline 107 & 1 & 10 \\
\hline 107 & 2 & 18 \\
\hline 107 & 3 & 6 \\
\hline 107 & 4 & 16 \\
\hline 107 & 5 & 21 \\
\hline 107 & 6 & 24 \\
\hline 107 & 7 & 27 \\
\hline 107 & 8 & 17 \\
\hline 111 & 1 & --- \\
\hline 111 & 2 & --- \\
\hline 111 & 3 & --- \\
\hline 111 & 4 & 19 \\
\hline 111 & 5 & 32 \\
\hline 111 & 6 & 34 \\
\hline 111 & 7 & 26 \\
\hline 111 & 8 & 31 \\
\hline
\end{tabular}

\begin{tabular}{|c|c|c|}
\hline 101 & 1 & --- \\
\hline 101 & 2 & --- \\
\hline 101 & 3 & 25 \\
\hline 101 & 4 & 35 \\
\hline 101 & 5 & 22 \\
\hline 101 & 6 & 7 \\
\hline 101 & 7 & 9 \\
\hline 101 & 8 & 8 \\
\hline 103 & 1 & --- \\
\hline 103 & 2 & --- \\
\hline 103 & 3 & 30 \\
\hline 103 & 4 & 20 \\
\hline 103 & 5 & 33 \\
\hline 103 & 6 & 28 \\
\hline 103 & 7 & 23 \\
\hline 103 & 8 & 29 \\
\hline
\end{tabular}

Tracker No.2

\begin{tabular}{|c|c|c|}
\hline 109 & 1 & 48 \\
\hline 109 & 2 & --- \\
\hline 109 & 3 & 57 \\
\hline 109 & 4 & 60 \\
\hline 109 & 5 & 47 \\
\hline 109 & 6 & 50 \\
\hline 109 & 7 & 55 \\
\hline 109 & 8 & --- \\
\hline 104 & 1 & 49 \\
\hline 104 & 2 & 54 \\
\hline 104 & 3 & 53 \\
\hline 104 & 4 & 44 \\
\hline 104 & 5 & 40 \\
\hline 104 & 6 & --- \\
\hline 104 & 7 & 42 \\
\hline 104 & 8 & 43 \\
\hline
\end{tabular}

\begin{tabular}{|c|c|c|}
\hline 102 & 1 & 37 \\
\hline 102 & 2 & --- \\
\hline 102 & 3 & 36 \\
\hline 102 & 4 & 52 \\
\hline 102 & 5 & --- \\
\hline 102 & 6 & 46 \\
\hline 102 & 7 & 45 \\
\hline 102 & 8 & 56 \\
\hline 110 & 1 & --- \\
\hline 110 & 2 & 58 \\
\hline 110 & 3 & 41 \\
\hline 110 & 4 & 51 \\
\hline 110 & 5 & 59 \\
\hline 110 & 6 & 38 \\
\hline 110 & 7 & 39 \\
\hline 110 & 8 & --- \\
\hline
\end{tabular}

Figure 7.15: Connection table of external light-guides with D0 connectors on the cassettes. Left two boxes are for Tracker No.1 and right-hand two boxes are for Tracker No.2. First column is cassette number, second column is location of D0 connector and third column is external light-guide number 
Tracker No.1

\begin{tabular}{|l|l|l|l|l|l|}
\hline & Station1 & Station2 & Station3 & Station4 & Station5 \\
\hline Bulkhead 1 & 26 & 31 & 21 & 16 & 10 \\
\hline Bulkhead 2 & 27 & 32 & 22 & 17 & 8 \\
\hline Bulkhead 3 & 28 & 33 & 23 & 18 & 6 \\
\hline Bulkhead 4 & 29 & 34 & 24 & 19 & 7 \\
\hline Bulkhead 5 & 30 & 35 & 25 & 20 & 9 \\
\hline
\end{tabular}

Tracker No.2

\begin{tabular}{|l|l|l|l|l|l|}
\hline & Station1 & Station2 & Station3 & Station4 & Station5 \\
\hline Bulkhead 1 & 56 & 51 & 46 & 41 & 36 \\
\hline Bulkhead 2 & 57 & 52 & 47 & 42 & 37 \\
\hline Bulkhead 3 & 58 & 53 & 48 & 43 & 38 \\
\hline Bulkhead 4 & 59 & 54 & 49 & 44 & 39 \\
\hline Bulkhead 5 & 60 & 55 & 50 & 45 & 40 \\
\hline
\end{tabular}

Figure 7.16: Connection table of internal light-guides with station connectors for Tracker No.1 and No.2. Bulkhead number in the first column corresponds to the location in the station defined in Figure 6.1. Six optical connectors of internal light-guides are attached with station connectors in defined order. 


$$
y_{\text {tri }}=y_{v}=y_{w}
$$

, where $x_{v}, x_{w}, y_{v}, y_{w}$ are defined as follows.

$$
\begin{aligned}
& y_{v}=y_{w}=x_{d i s}, \text { and } \\
& x_{v}=\left(y_{v}-v_{d i s} / \cos \left(\theta_{v}\right)\right) / \tan \left(\theta_{v}\right), \text { and } \\
& x_{w}=\left(y_{w}-w_{d i s} / \cos \left(\theta_{w}\right)\right) / \tan \left(\theta_{w}\right), \text { and } \\
& v_{d i s}=p_{c}\left(f_{v}-f_{v 0}\right), \text { and } \\
& x_{d i s}=p_{c}\left(f_{x}-f_{x 0}\right), \text { and } \\
& w_{d i s}=p_{c}\left(f_{w}-f_{w 0}\right)
\end{aligned}
$$

where $v_{d i s}, x_{d i s}$ and $w_{d i s}$ are distances from center fibers in their views. $f_{v}, f_{x}$, and $f_{w}$ are the hit fiber numbers and $f_{v 0}, f_{x 0}$ and $f_{w 0}$ are the center fibers of the $\mathrm{V}, \mathrm{X}$ and $\mathrm{W}$ views. $\theta_{v}$ and $\theta_{w}$ are rotational angles of the $\mathrm{V}$ and $\mathrm{W}$ view with respect to the $\mathrm{X}$ view. $p_{c}$ is pitch of channel (1494.5 $\mu m$ ), which is 3.5 times of $p_{f}$ fiber pitch $(427 \mu m)$.

If views are aligned at designed positions and angles, distance between $x_{v}$ and $x_{w}, \Delta x_{v w}$ is;

$$
\Delta x_{v w}=p_{c}\left(f_{v}+f_{x}+f_{w}\right)-p_{c}\left(f_{v 0}+f_{x 0}+f_{w 0}\right)
$$

If thickness of fiber is not considered, triplet is identified when $\Delta x_{v w}$ equals 0. If thickness of fiber is considered, triplet has multi-peak on $\Delta x_{v w}$ as shown in Figure 7.17.

The measured distribution of $\Delta x_{v w}$ for Tracker No.1 and No.2 are shown in Figure 7.18. By Figure 7.18, triplets on each stations are identified.

In all stations except for Station No.5 of Tracker No.1, similar distributions to the plot of $-3 p_{f}$ in Figure 7.17) are seen. It is considered that one of views in Station No.5 of Tracker No.1 are glued off by about one channel or so. The position of views are corrected in the next step.

\subsubsection{Correcting misalignments}

Misalignments of views and stations are corrected in the way as described below. Here two coordinates are defined as follows.

\section{Station coordinate}

Station coordinate is a two dimensional ( $\mathrm{x}-\mathrm{y})$ coordinate, where origin is defined by the intersection of the center fiber of $\mathrm{V}$ and $\mathrm{X}$ view, which is 106 . $\mathrm{Y}$ axis corresponds to the center fiber of the $\mathrm{X}$ view and $\mathrm{X}$ axis is vertical to the $\mathrm{Y}$ axis.

In the station coordinate, rotational angles of $\mathrm{V}$ and $\mathrm{W}$ view with respect to $\mathrm{X}$ view $\left(\theta_{v}, \theta_{w}\right)$ are corrected for five stations.

\section{Tracker coordinate}

Tracker coordinate is three dimensional $(x-y-z)$ coordinate. X-Y coordinate in the tracker coordinate is defined by Station No.1. Z axis is defined by the vector between two origins of the outermost stations No.1 and No.5.

In the tracker coordinate, origins of middle three stations $\left(x_{20}, y_{20}, x_{30}, y_{30}, x_{40}, y_{40}\right)$ and rotational angles of four stations, No.2, No.3, No.4 and No.5 $\left(\theta_{2}, \theta_{3}, \theta_{4}, \theta_{5}\right)$ with respect to Station No.1 are corrected. 
$-4 \mathrm{Pf}$

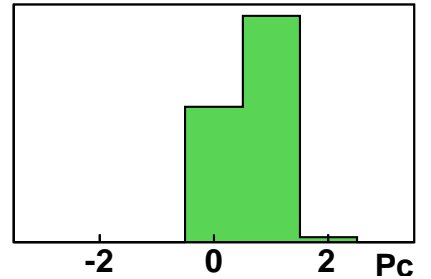

$-1 \mathrm{Pf}$

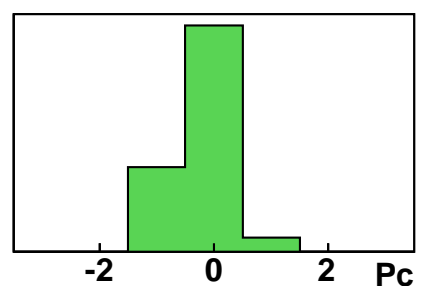

$2 \mathrm{Pf}$

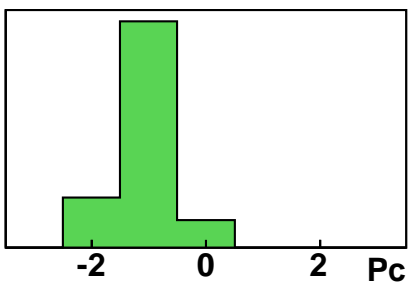

-3 Pf

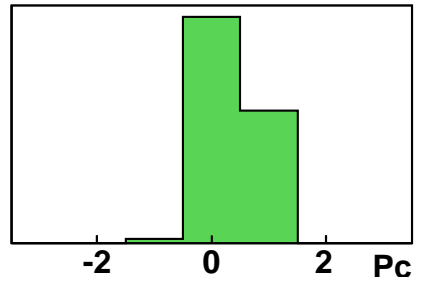

0 Pf

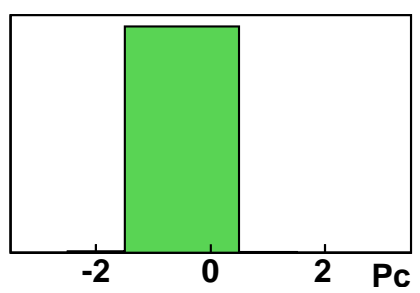

3 Pf

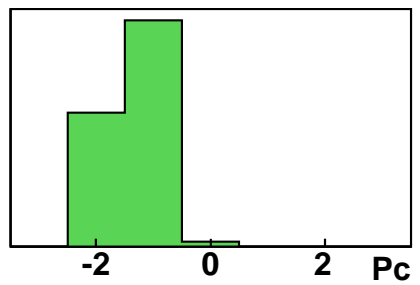

$-2 \mathrm{Pf}$

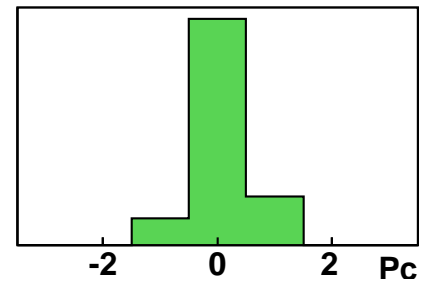

$1 \mathrm{Pf}$

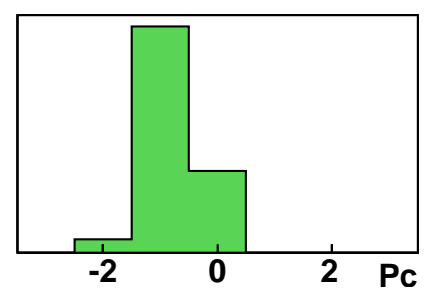

4 Pf

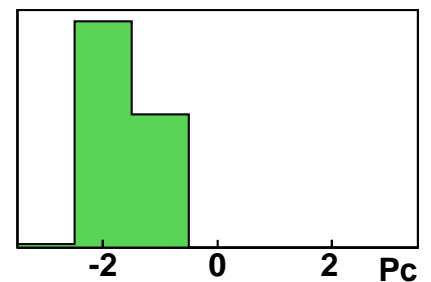

Figure 7.17: Expected $\Delta x_{v w}$ distributions by simulation with different positions of center fiber of $\mathrm{X}$ view. Displacement of $\mathrm{X}$ view is shown on top of each plot.

Tracker1
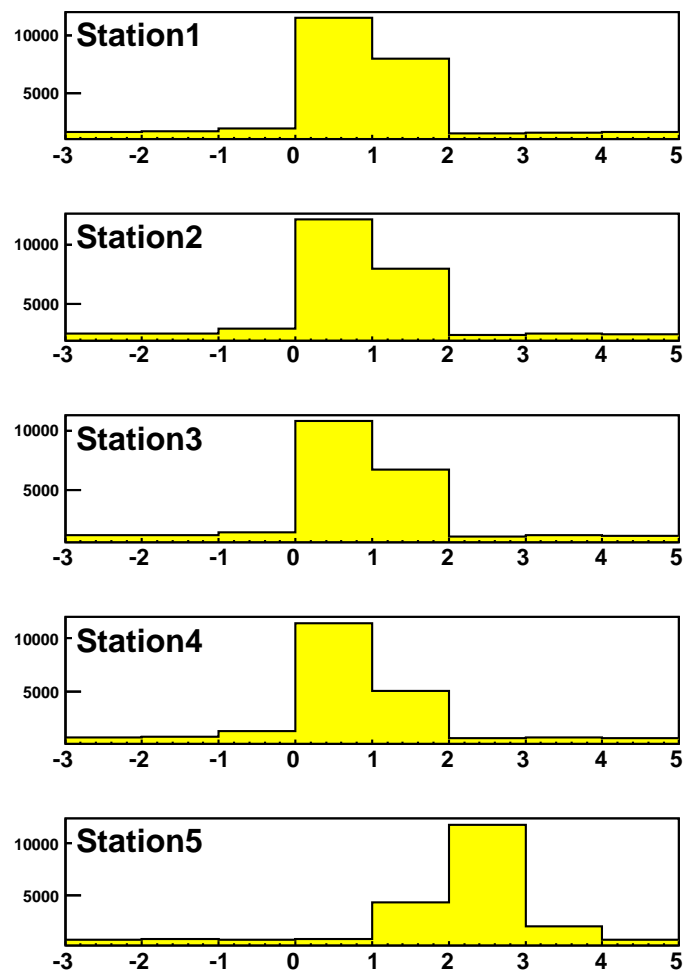

\section{Tracker2}
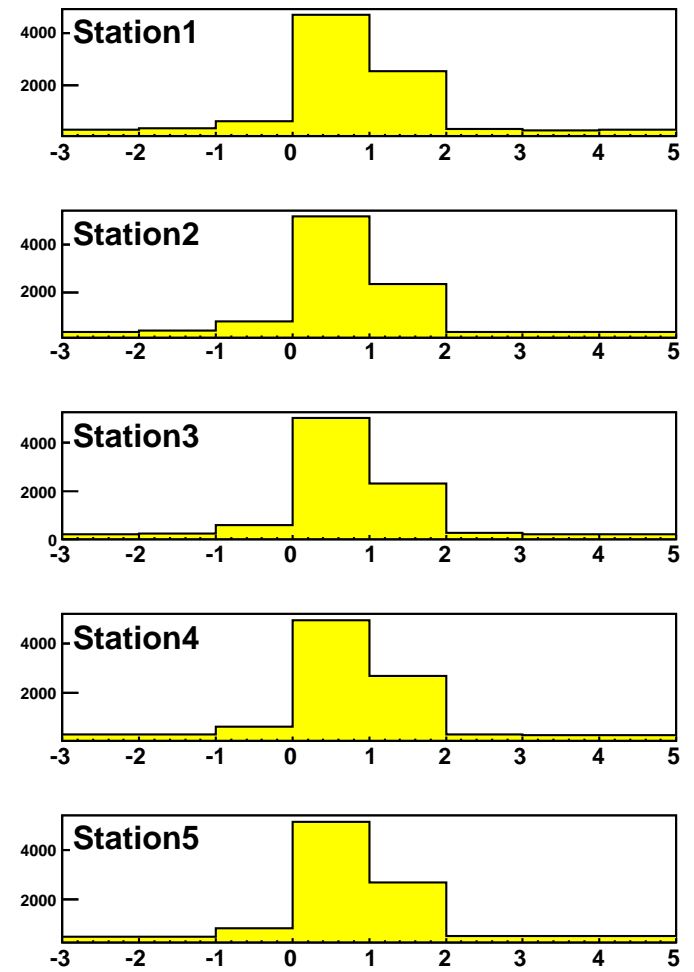

Figure 7.18: Measured $\Delta x_{v w}$ distributions at Tracker No.1 (left) and No.2 (right). 


\subsubsection{Correcting misalignments of views}

Considering the rotational misalignment of $\mathrm{V}$ and $\mathrm{W}$ view, $d v$ and $d w$ with respect to $\mathrm{X}$ view in unit of radian, equation 7.9 changes to;

$$
\Delta x_{v w}^{\prime}=\Delta x_{v w}+f_{x}\left(\frac{2}{\sqrt{3}} d v-\frac{2}{\sqrt{3}} d w\right)+f_{v} \frac{d v}{\sqrt{3}}-f_{w} \frac{d w}{\sqrt{3}}
$$

where $\Delta x_{v w}^{\prime}$ is $\Delta x_{v w}$ after the correction.

Measured $\Delta x_{v w}$ as a function of fiber number for Tracker No.1 is shown in Figure 7.19. Station No.1, No.2 and No.5 are corrected. The best angles of $\mathrm{V}$ and $\mathrm{W}$ views were searched to change angles in 0.1 degree steps until no correlation is found. $\Delta x_{v w}^{\prime}$ for Tracker No.1 is shown in Figure 7.19 .

Measured $\Delta x_{v w}$ as a function of fiber number for Tracker No.2 is shown in Figure 7.20. No correction is made for Tracker No.2.

The correction parameters on views for Tracker No.1 and No.2 are summarized in Table 7.1.

\begin{tabular}{cccc}
\hline Tracker & Station & $\mathrm{V}(\mathrm{deg})$ & $\mathrm{W}(\mathrm{deg})$ \\
\hline 1 & 1 & 239.9 & 120.0 \\
1 & 2 & 240.1 & 120.1 \\
1 & 3 & 240.0 & 120.0 \\
1 & 4 & 240.0 & 120.0 \\
1 & 5 & 239.8 & 119.8 \\
\hline 2 & 1 & 240.0 & 120.0 \\
2 & 2 & 240.0 & 120.0 \\
2 & 3 & 240.0 & 120.0 \\
2 & 4 & 240.0 & 120.0 \\
2 & 5 & 240.0 & 120.0 \\
\hline
\end{tabular}

Table 7.1: Correction parameters on views for Tracker No.1 and No.2

\subsubsection{Correcting misalignments of stations}

Corrections of stations are independently made by checking residuals between hit position and interpolated position at middle three stations. The interpolated position is estimated from linear tracks made by two hit position at station No.1 and No.5.

The $\mathrm{x}$, y residuals at the station number $i,\left(\Delta x_{i}, \Delta y_{i}\right)$ have relationship with rotation angles and $\mathrm{x}, \mathrm{y}$ offsets of stations as follows;

$$
\begin{aligned}
& \Delta x_{i}=\left(y_{2}-y_{i 0}\right) \theta_{2}-\alpha\left(y_{5}-y_{50}\right) \theta_{5} \\
& \Delta y_{i}=\left(x_{2}-x_{i 0}\right) \theta_{2}-\alpha\left(x_{5}-x_{50}\right) \theta_{5} \\
& \Delta x_{5}=\left(y_{5}-y_{50}\right) \theta_{5}-\beta\left(y_{i}-y_{i 0}\right) \theta_{i} \\
& \Delta y_{5}=\left(x_{5}-x_{50}\right) \theta_{5}-\beta\left(x_{i}-x_{i 0}\right) \theta_{i} \\
& \alpha=\frac{z_{5}-z 1}{z 1-z_{i}} \\
& \beta=\frac{z_{5}-z 1}{z 1-z_{i}}
\end{aligned}
$$




\section{Tracker1}
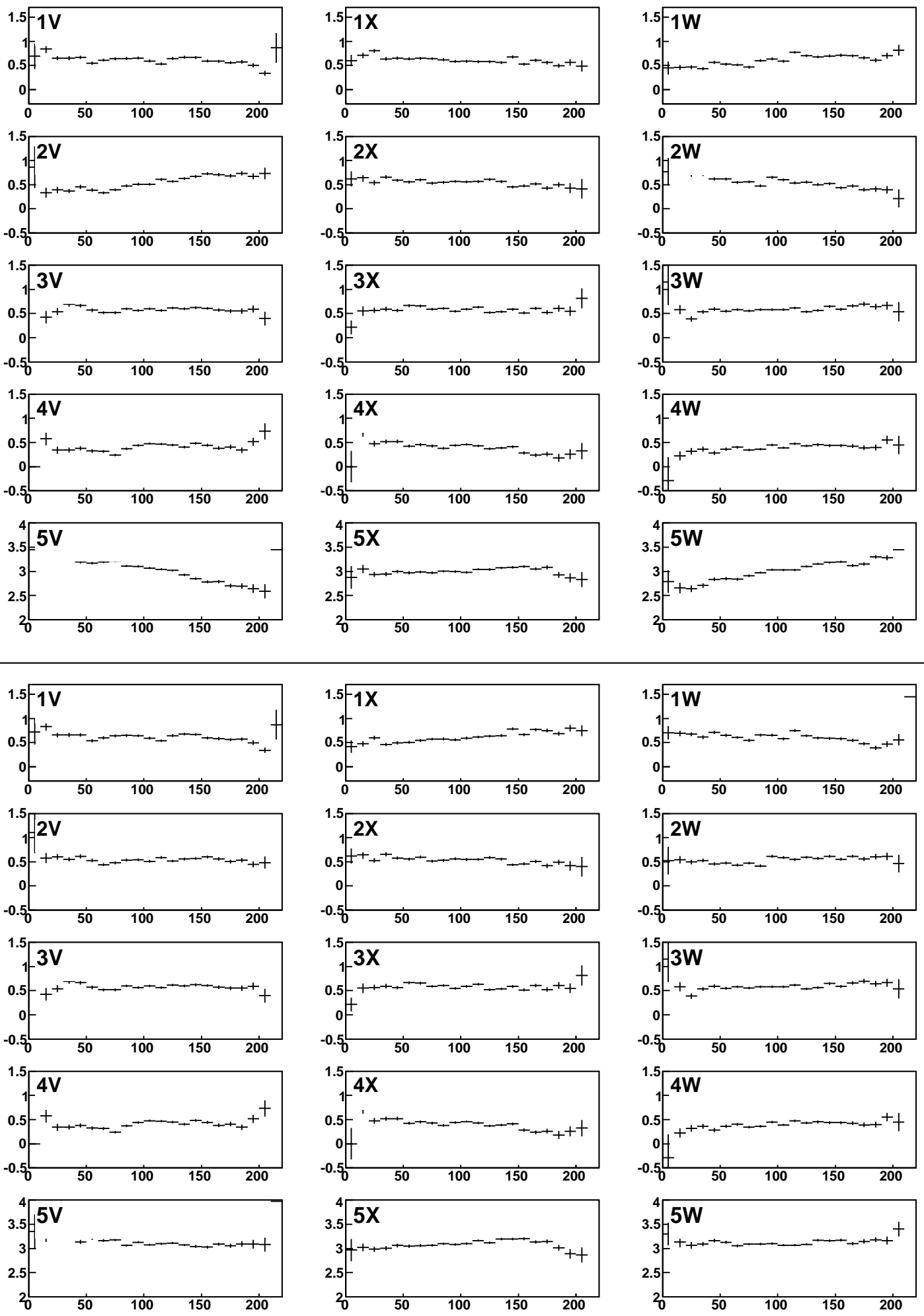

Figure 7.19: Measured $\Delta x_{v w}$ (top) and corrected $\Delta x_{v w}$ (bottom) for Tracker No.1. Alignments of Station No.1, No.2 and No.5 are corrected. 


\section{Tracker2}
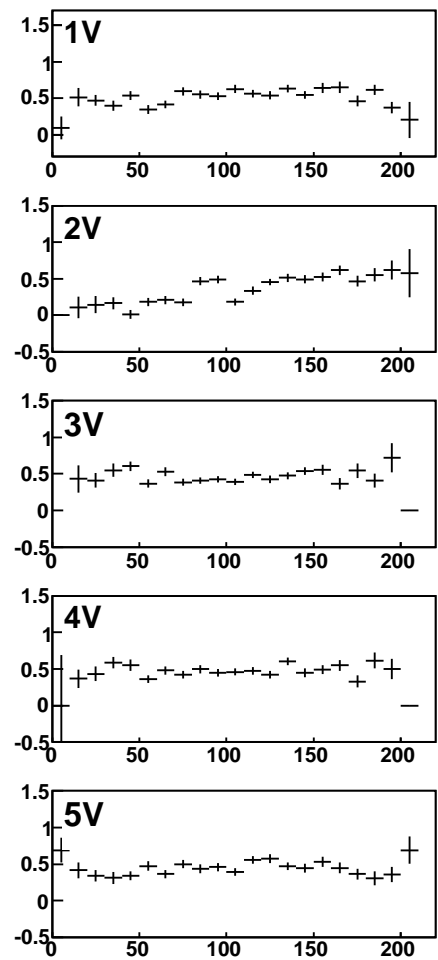
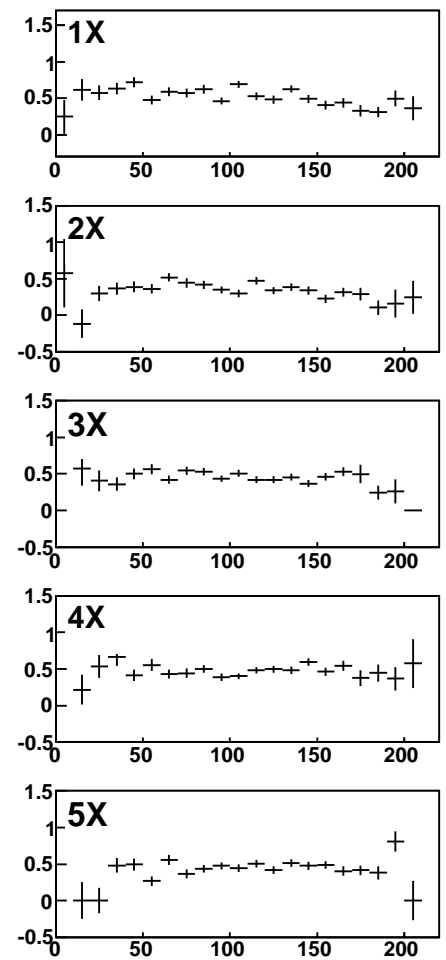
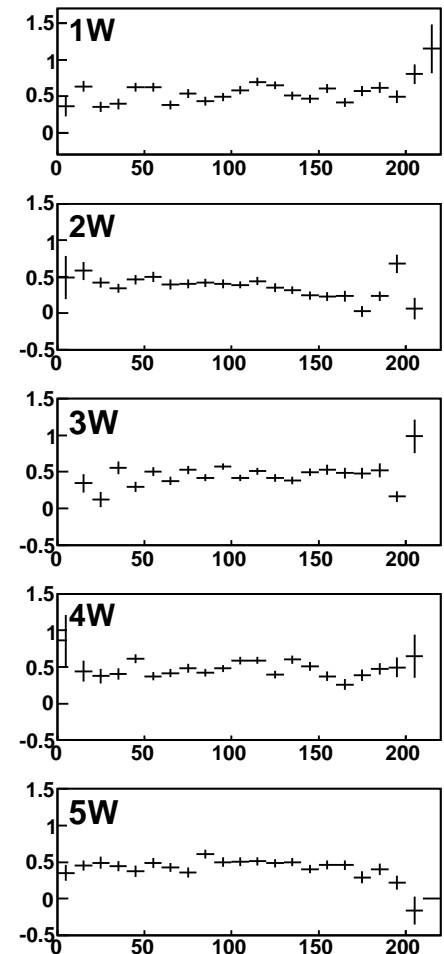

Figure 7.20: Measured $\Delta x_{v w}$ distribution for Tracker No.2. No correction is made for Tracker No.2. 
, where $i$ is station number of either 2,3 or 4 and $x_{i 0}$ and $y_{y 0}$ are $\mathrm{x}, \mathrm{y}$ offsets of the station number $i$.

The best angles are firstly searched in 0.1 degree steps until no correlations are found, and then the $\mathrm{x}$, $\mathrm{y}$ offsets are calculated. The measured and corrected residuals as a function of hit position for Tracker No.1 and No.2 are shown in Figure 7.21 and Figure 7.22, respectively. The station offsets and angles thus determined are summarized in Table 7.2.

\begin{tabular}{ccccc}
\hline Tracker & Station & $x_{0}(\mathrm{~mm})$ & $y_{0}(\mathrm{~mm})$ & $\theta(\mathrm{deg})$ \\
\hline 1 & 1 & 0 & 0 & 0 \\
1 & 2 & 0.43 & -0.61 & -0.2 \\
1 & 3 & 0.28 & -1.43 & -0.2 \\
1 & 4 & 0.34 & -1.94 & -0.2 \\
1 & 5 & 0 & 0 & 0.0 \\
\hline 2 & 1 & 0 & 0 & 0 \\
2 & 2 & 0.11 & -0.68 & 0.1 \\
2 & 3 & -0.01 & -0.90 & 0.2 \\
2 & 4 & -0.01 & -0.33 & 0.2 \\
2 & 5 & 0 & 0 & 0.2 \\
\hline
\end{tabular}

Table 7.2: Correction parameters on stations for Tracker No.1 and No.2

\subsection{Performance}

Once misalignments of views and stations are corrected, alignments of fibers are checked by comparing residuals with these estimated by Monte-Carlo simulation. Hit efficiencies and triplet efficiencies are examined for all the views of all the stations.

\subsubsection{Alignment}

To calculate positions at a station under test, linear tracks are made using hit positions on four stations except for the station under test. The offset and tilt of linear tracks are determined in a way that residuals become minimum, then residuals are calculated by subtracting hit positions and interpolating (extrapolating) positions.

Following expected resolutions calculated by Monte-Carlo simulation are described, measured residuals at Tracker No.1 and No.2 are explained.

\subsubsection{Residuals expected by Monte-Carlo simulation}

If interpolated (extrapolated) positions (referred to as fitted position) has no errors, residuals of $\mathrm{x}$ and $\mathrm{y}$ hit position are expected as shown in Figure 7.23. But linear tracks are made from hit positions which are digitized by channel pitch, $p_{c}$, there are some resolutions on fitted positions. To estimate these resolutions, residuals of interpolated (extrapolated) positions and true hit position at five stations are examined as shown in Figure 7.24. Increase of errors on fitted position are seen at the outermost station No.1 and No.5. Expected residual distributions to be compared with data 
Tracker1

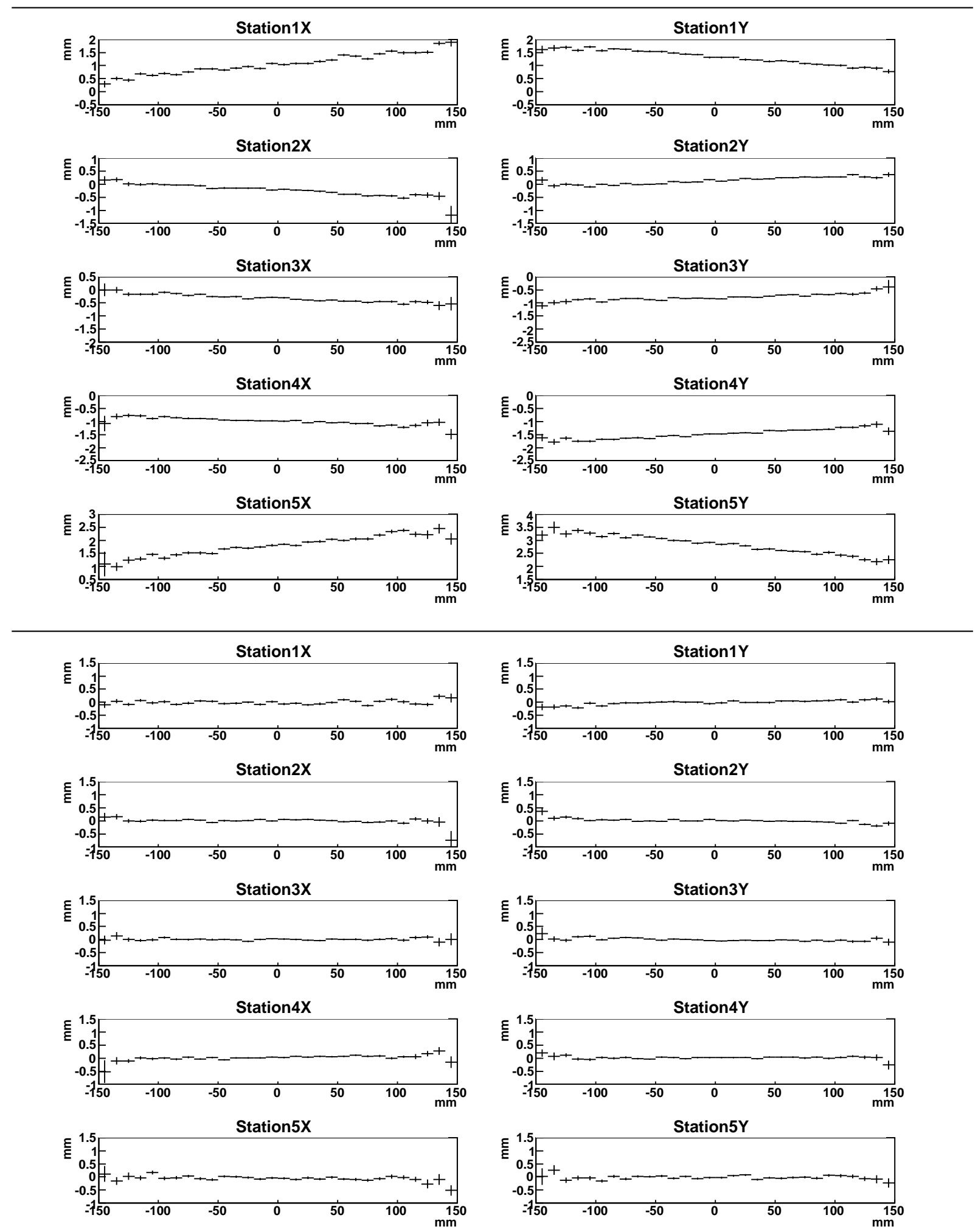

Figure 7.21: Measured $\Delta x(y)_{i}$ vs $y(x)_{i}$ (top) and corrected $\Delta x(y)_{i}$ vs $y(x)_{i}$ (bottom) for Tracker No.1. 


\section{Tracker2}
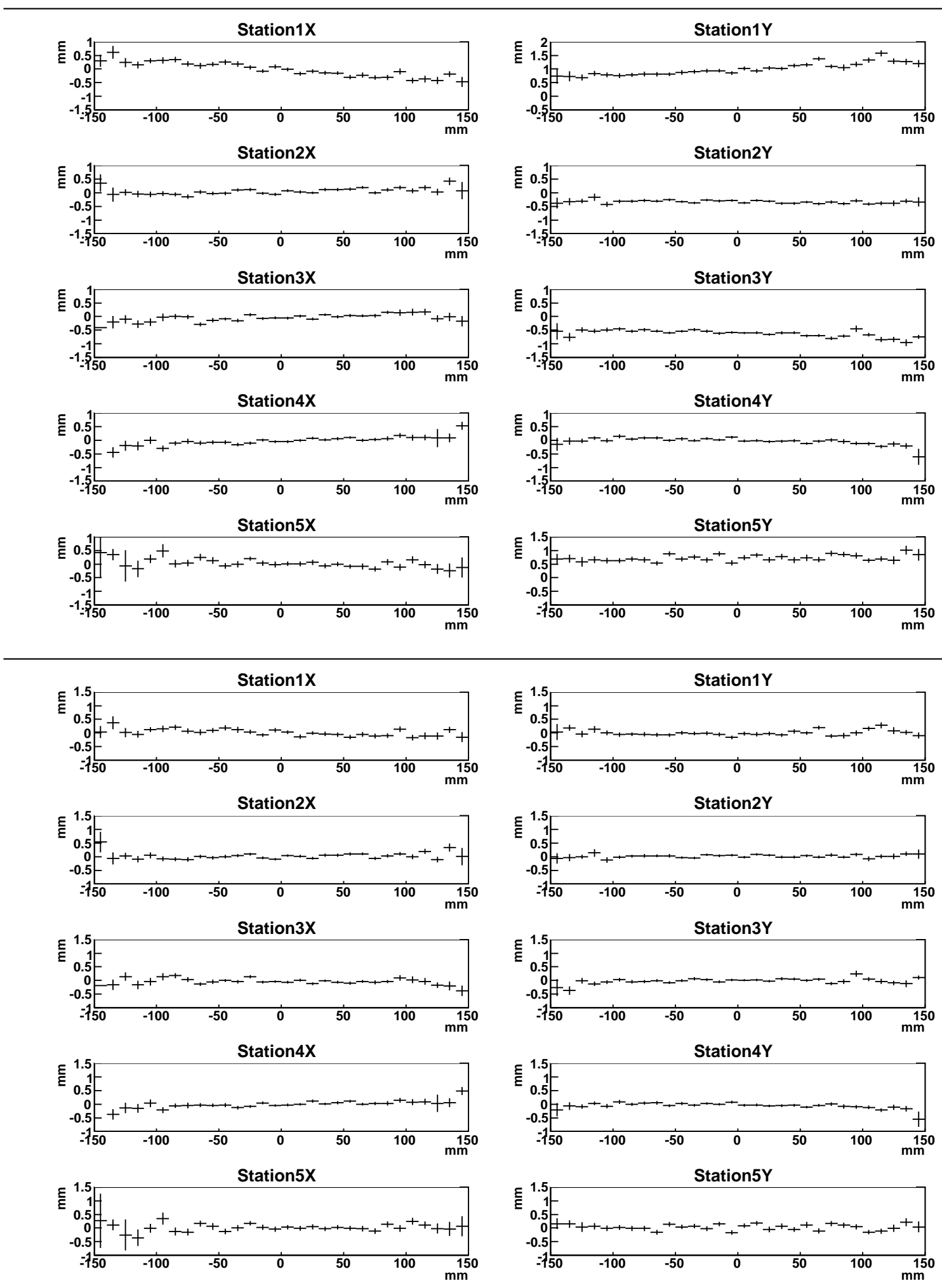

Figure 7.22: Measured $\Delta x(y)_{i}$ vs $y(x)_{i}$ (top) and corrected $\Delta x(y)_{i}$ vs $y(x)_{i}$ (bottom) for Tracker No.2. 
at five stations are shown in Figure 7.25. Expected RMS (Sigma) of residuals are summarized at 'Res' column in Table 7.3.
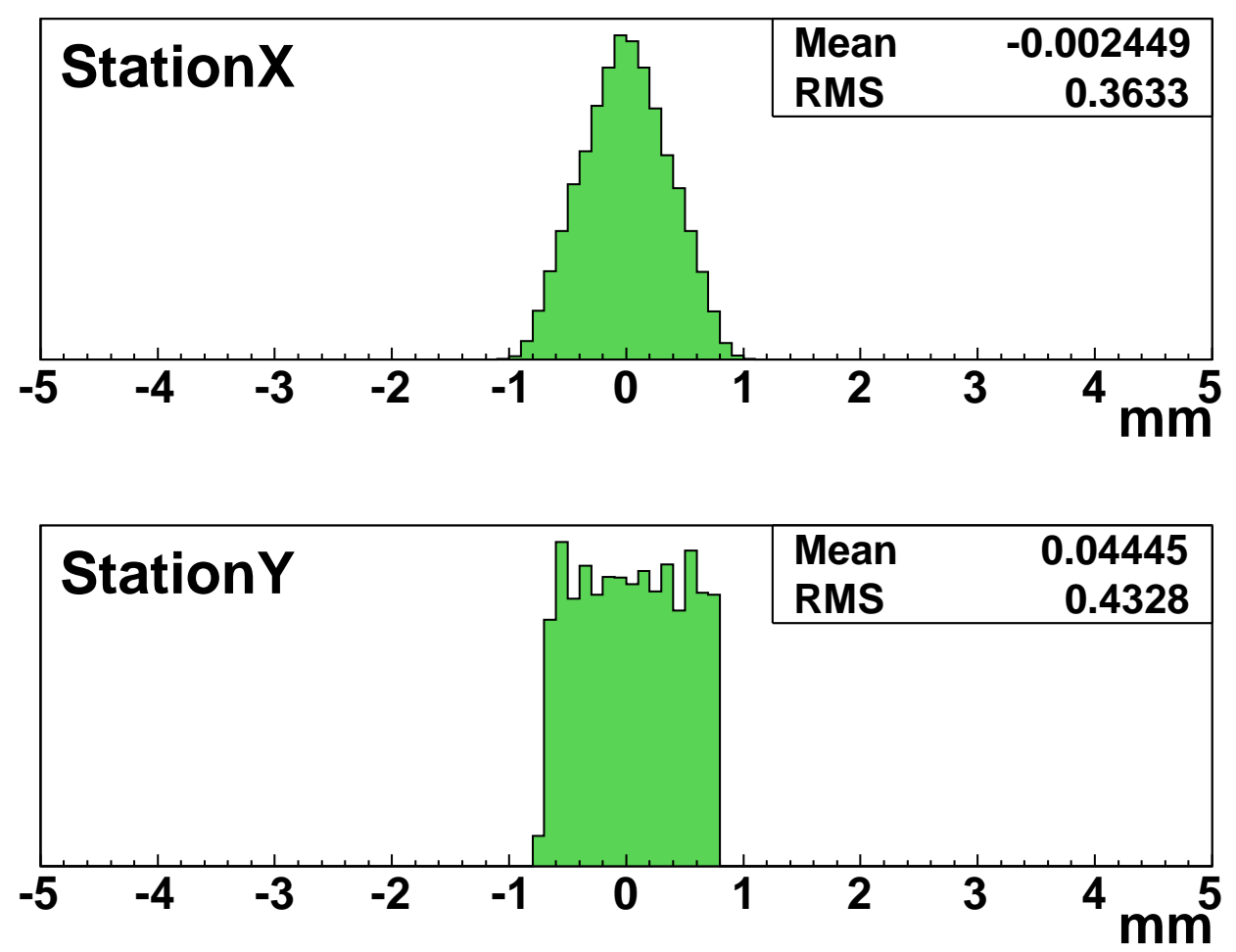

Figure 7.23: Residuals of hit positions calculated from fiber number and true hit position for $\mathrm{X}$ axis (top) and $\mathrm{Y}$ axis(bottom).

\subsubsection{Residuals measured by cosmic-ray data}

Measured residual distribution at five stations of Tracker No.1 and No.2 are shown in Figure 7.26. Measured sigmas of residuals are summarized with expectations from simulations in Table 7.4. Measured alignments have agreements with results by simulations within about $50 \mu \mathrm{m}$ for both trackers.

\subsubsection{Efficiency}

Hit efficiency of individual fibers are examined. Test fiber is searched around the fitted position and identified by the fiber whose light-yield is maximum in searched fibers. Hit fibers and these light-yield distributions on views are shown in Figure 7.27 and Figure 7.29, respectively. The small light-yields found at X view of Station No.5 is discussed in the next section 7.6.

Measured hit efficiency as a function of fiber number for Tracker No.1 and No.2 are shown in Figure 7.28. Measured hit efficiencies averaged in each views are summarized in Table 7.5. In this calculation, fibers in the middle region of the station (fiber number from 30 to 180) are used. 

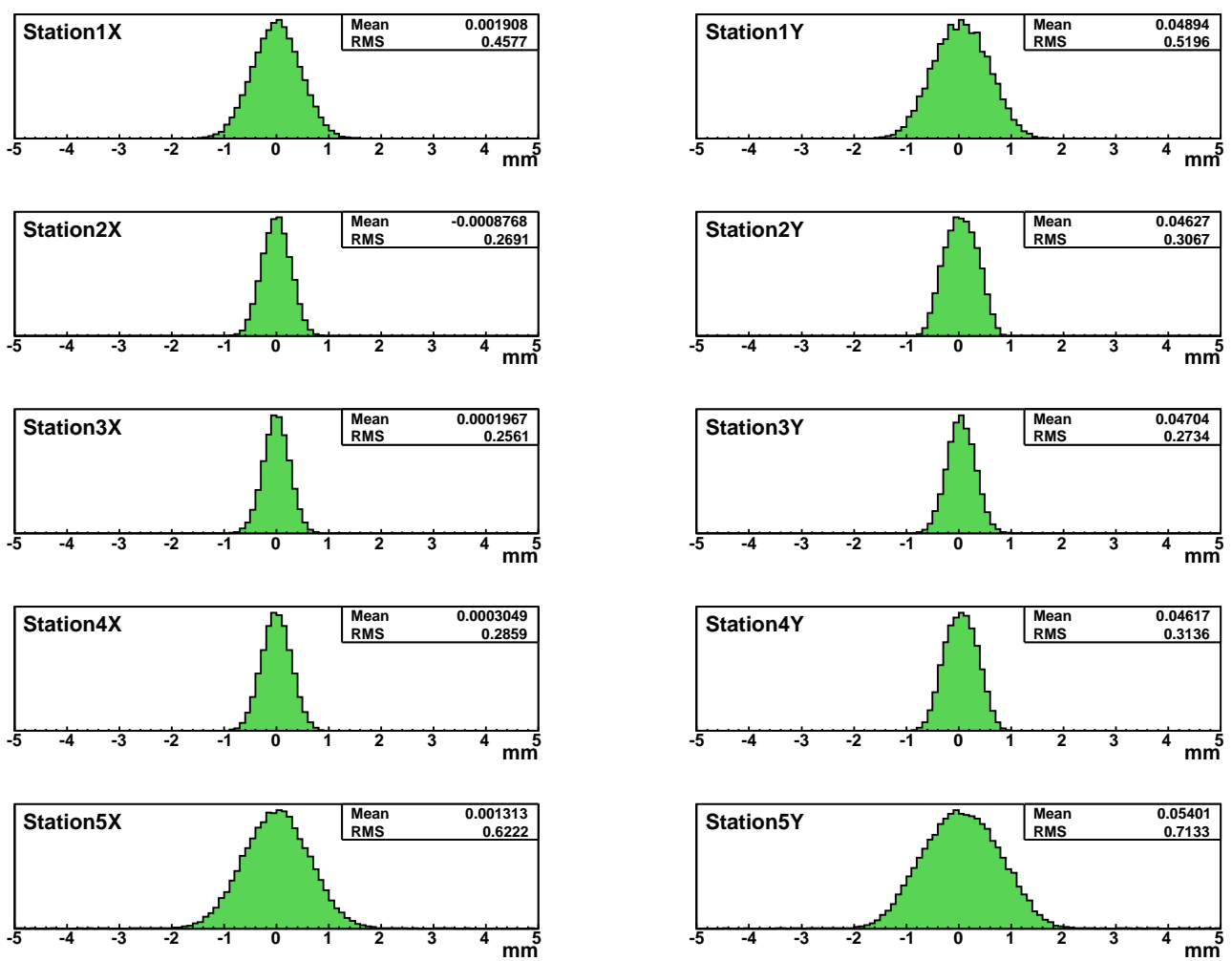

Figure 7.24: Residuals of interpolated (extrapolated) positions and true hit position.
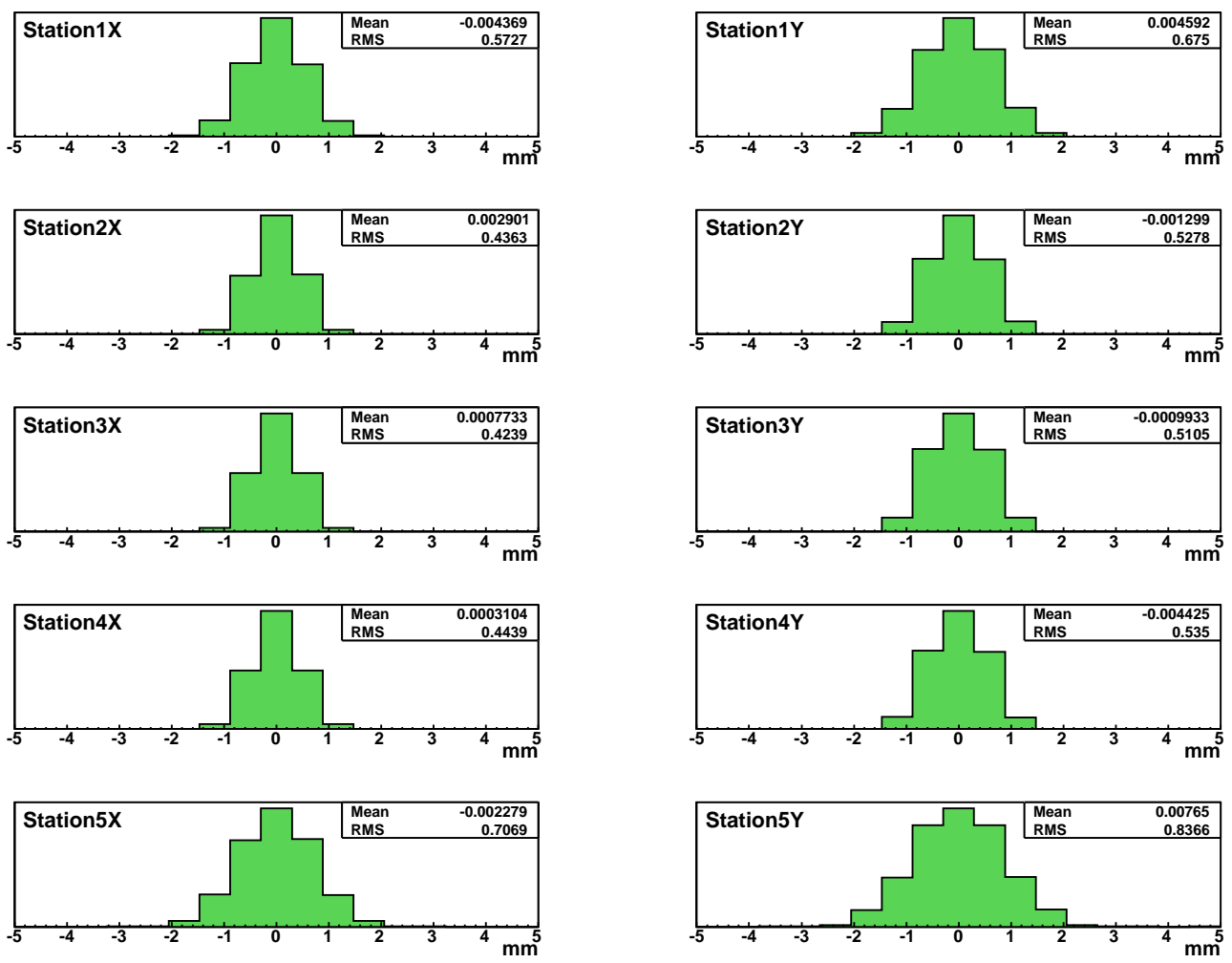

Figure 7.25: Residuals of interpolated (extrapolated) positions and hit position calculated from fiber number. 


\begin{tabular}{cccc}
\hline Station $(\mathrm{X} / \mathrm{Y})$ & Hit $(\mathrm{mm})$ & Fit $(\mathrm{mm})$ & Res $(\mathrm{mm})$ \\
\hline $1 \mathrm{X}$ & 0.363 & 0.457 & $0.573(0.587)$ \\
$2 \mathrm{X}$ & 0.363 & 0.269 & $0.436(0.460)$ \\
$3 \mathrm{X}$ & 0.363 & 0.256 & $0.424(0.457)$ \\
$4 \mathrm{X}$ & 0.363 & 0.286 & $0.444(0.471)$ \\
$5 \mathrm{X}$ & 0.363 & 0.622 & $0.707(0.720)$ \\
\hline $1 \mathrm{Y}$ & 0.433 & 0.520 & $0.675(0.679)$ \\
$2 \mathrm{Y}$ & 0.433 & 0.307 & $0.528(0.516)$ \\
$3 \mathrm{Y}$ & 0.433 & 0.273 & $0.511(0.544)$ \\
$4 \mathrm{Y}$ & 0.433 & 0.314 & $0.535(0.534)$ \\
$5 \mathrm{Y}$ & 0.433 & 0.713 & $0.837(0.841)$ \\
\hline
\end{tabular}

Table 7.3: Expected RMS of hit, fitted and residuals at five stations. The numbers in parentheses are sigmas fitted with Gaussian function.

\begin{tabular}{cccc}
\hline Station $(\mathrm{X} / \mathrm{Y})$ & $\sigma_{\text {tracker } 1}(\mathrm{~mm})$ & $\sigma_{\text {tracker } 2}(\mathrm{~mm})$ & $\sigma_{M C}(\mathrm{~mm})$ \\
\hline $1 \mathrm{X}$ & 0.662 & 0.644 & 0.587 \\
$2 \mathrm{X}$ & 0.498 & 0.488 & 0.460 \\
$3 \mathrm{X}$ & 0.493 & 0.489 & 0.457 \\
$4 \mathrm{X}$ & 0.506 & 0.499 & 0.471 \\
$5 \mathrm{X}$ & 0.816 & 0.795 & 0.720 \\
\hline $1 \mathrm{Y}$ & 0.744 & 0.733 & 0.679 \\
$2 \mathrm{Y}$ & 0.550 & 0.530 & 0.516 \\
$3 \mathrm{Y}$ & 0.558 & 0.548 & 0.544 \\
$4 \mathrm{Y}$ & 0.573 & 0.573 & 0.534 \\
$5 \mathrm{Y}$ & 0.909 & 0.887 & 0.841 \\
\hline
\end{tabular}

Table 7.4: Measure sigmas of residuals at five stations for Tracker No.1 and No.2. In the third column, sigma of residuals expected by simulation are shown. 

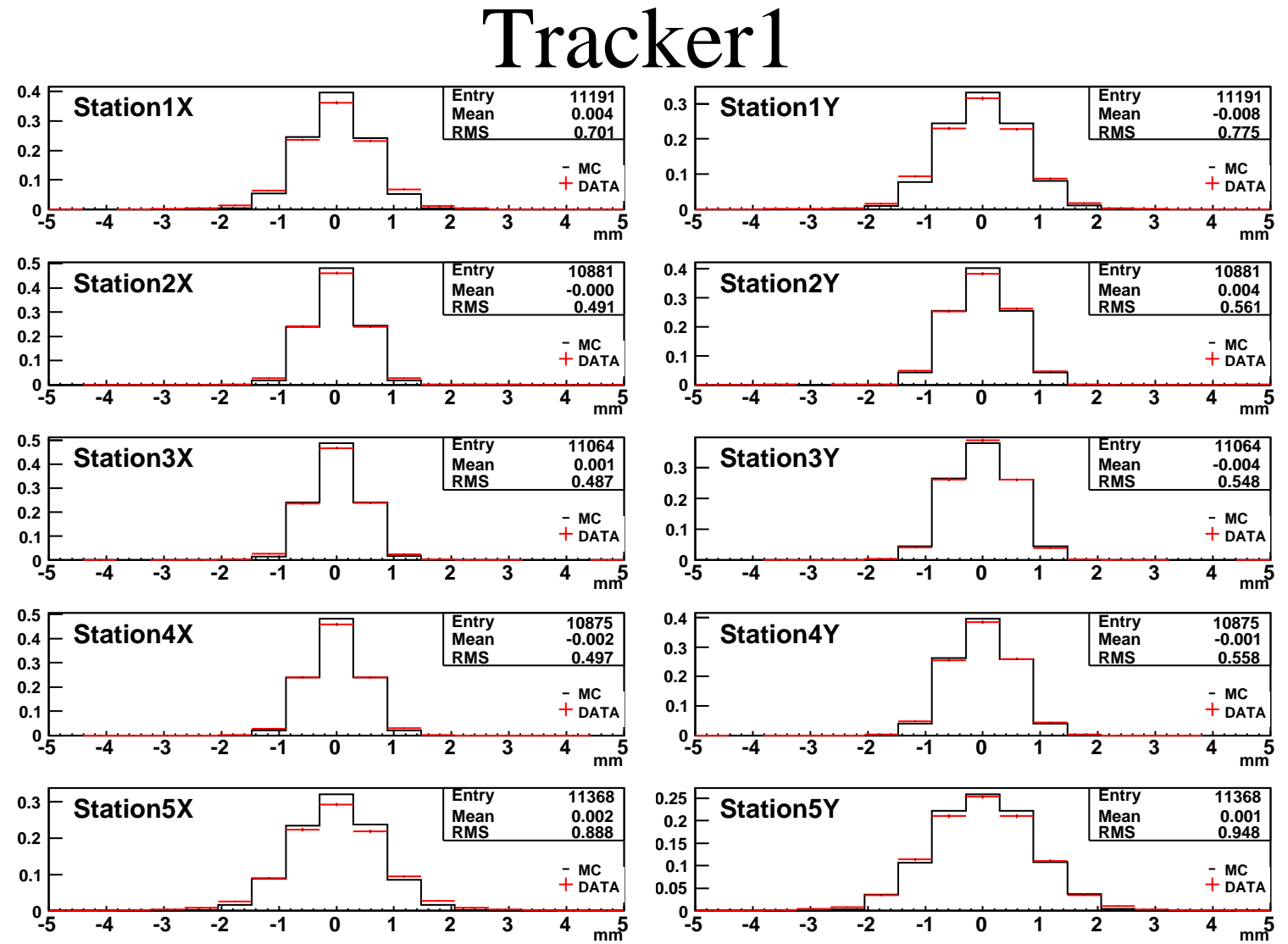

\section{Tracker2}
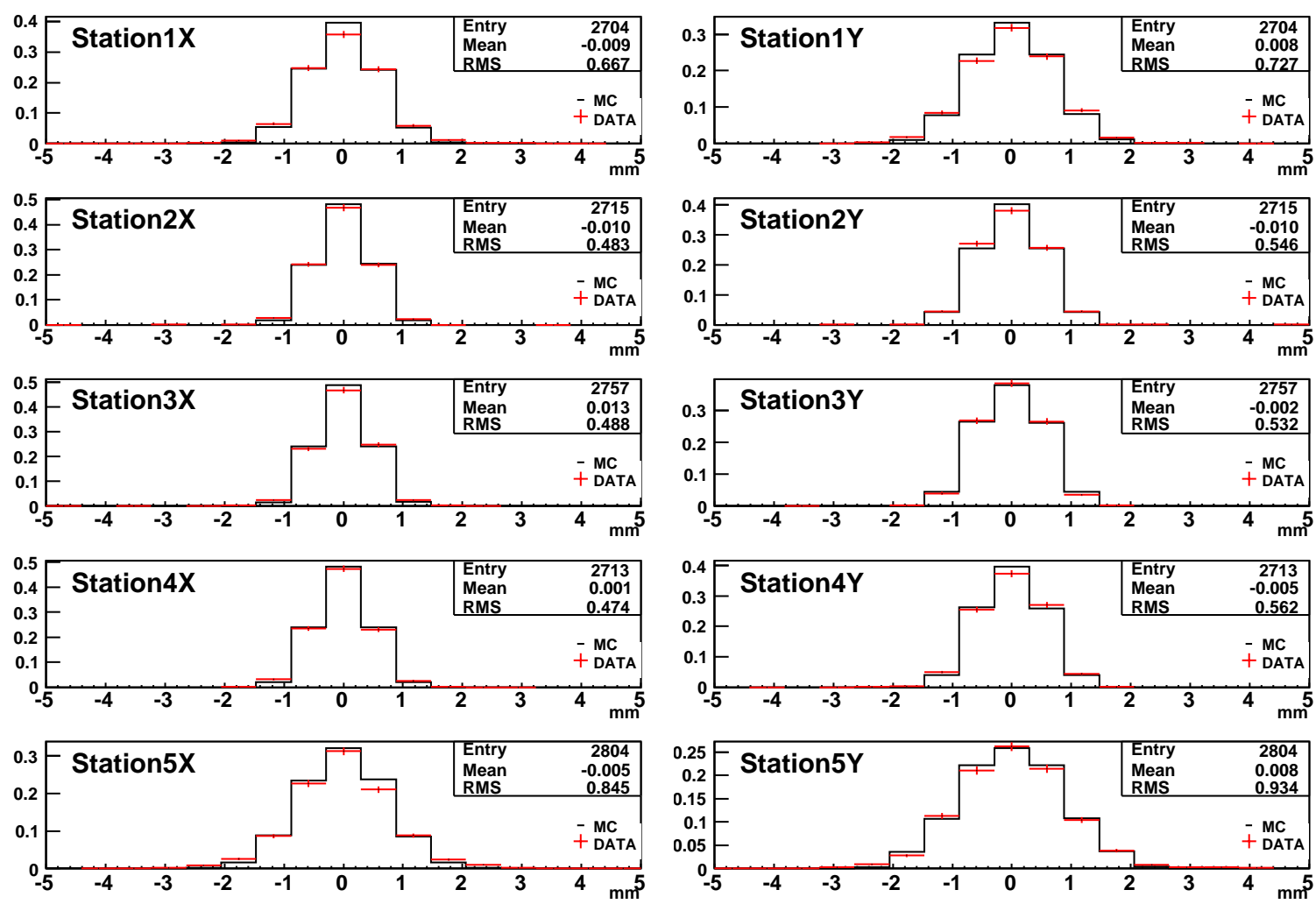

Figure 7.26: Measured residual distributions at Tracker No.1 (top) and No.2 (bottom). Data (red color with error bars) is superimposed with Monte-Carlo simulation (black histograms). 
Triplet efficiencies are calculated by the ratio of the number of triggers where triplets are found to the total number of triggers. In triplets, fractions of triplets on track (defined as triplets whose residuals are within $5 \mathrm{~mm}$ in this study) are shown in 'On track' column in Table 7.5. It is found that some of triplets are not on track, which is considered that these triplets are made by different cosmic-rays, that is, one cosmic-ray pass through top trigger counter without passing through bottom counter and the other cosmic-ray is the opposite case of this. Note that the fraction of fake triplets caused by VLPC noise is expected as to be less than $0.01 \%$, which is the expectation from a pedestal run.

Tracking efficiency is estimated by multiplying the triplet efficiencies (on track) of five stations. Tracking efficiency results in $91 \%$ for Tracker No.1 and $94 \%$ for Tracker No.2.

\begin{tabular}{ccccccc}
\hline Tracker & Station & HitV & HitX & HitW & Triplet & On track \\
\hline 1 & 1 & 0.997 & 0.998 & 0.997 & 0.992 & 0.978 \\
1 & 2 & 0.994 & 0.997 & 0.987 & 0.980 & 0.948 \\
1 & 3 & 0.992 & 0.992 & 0.992 & 0.976 & 0.965 \\
1 & 4 & 0.996 & 0.994 & 0.998 & 0.989 & 0.981 \\
1 & 5 & 0.998 & 0.982 & 0.995 & 0.973 & 0.965 \\
\hline 2 & 1 & 0.998 & 0.994 & 0.999 & 0.991 & 0.982 \\
2 & 2 & 0.998 & 0.998 & 0.996 & 0.993 & 0.983 \\
2 & 3 & 0.998 & 0.999 & 0.999 & 0.997 & 0.986 \\
2 & 4 & 0.998 & 0.997 & 0.999 & 0.995 & 0.987 \\
2 & 5 & 0.996 & 0.997 & 0.990 & 0.984 & 0.972 \\
\hline
\end{tabular}

Table 7.5: Measured efficiencies at Tracker No.1 and No.2.

\subsection{Discussion}

Small light-yields are found at the $\mathrm{X}$ views on the Station No.5 of the Tracker No.1 (Figure 7.29). These affects the hit efficiency to be reduced by about 1\% (Table 7.5), which is a small effect.

The distribution amount of the Light-yields distribution are made in terms of external lightguide number (Figure 7.30). Small light-yields (a peak is less than 10 photo-electrons) are seen at No.21, No.42 and No.52 as well as No.6.

Profile plots to examine the correlation between fiber number of light-guide and AFEIIt board used for the read-out are shown in Figure 7.31. It is turned out that light-guides No.21 and No.52 have correlation with AFEIIt board, and No.6 and No.42 have no correlation with board. The former suggests that the problem is on the board, not on the light-guides, while the latter suggests that the problem is on the light-guide or the board.

The problem can be investigated by swapping external light guides with others and checking the light-yields. In any cases, it can be solved by using spare external light-guides or using spare slots (D0 warm end). 


\section{Tracker1}
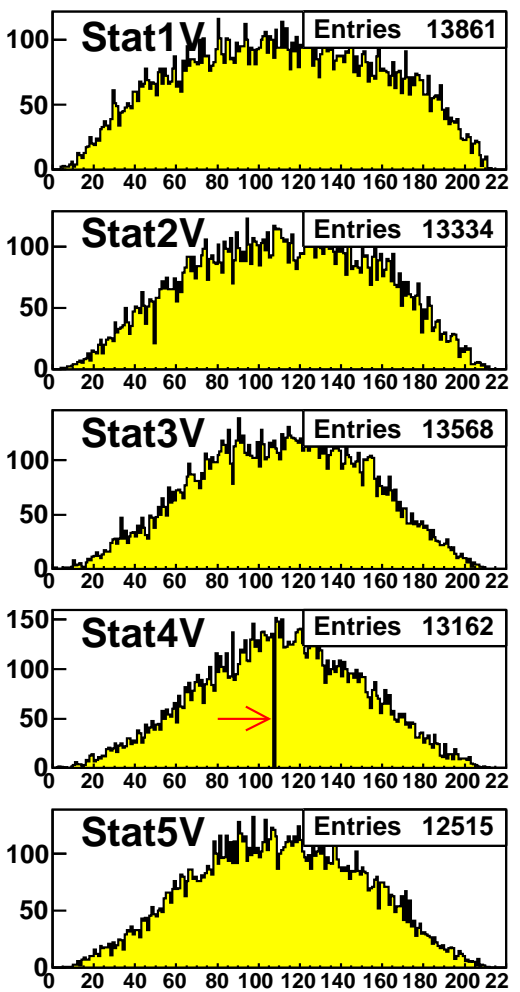

\section{Tracker2}
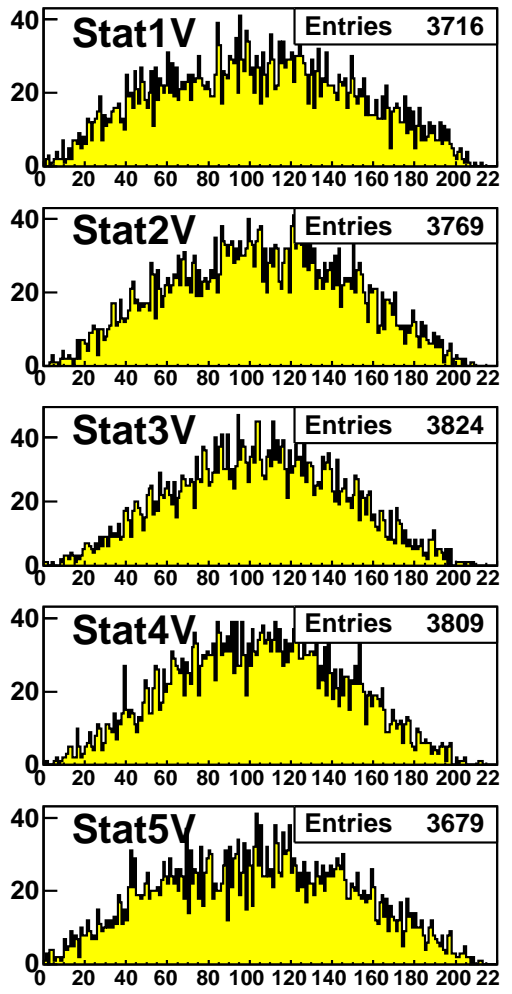
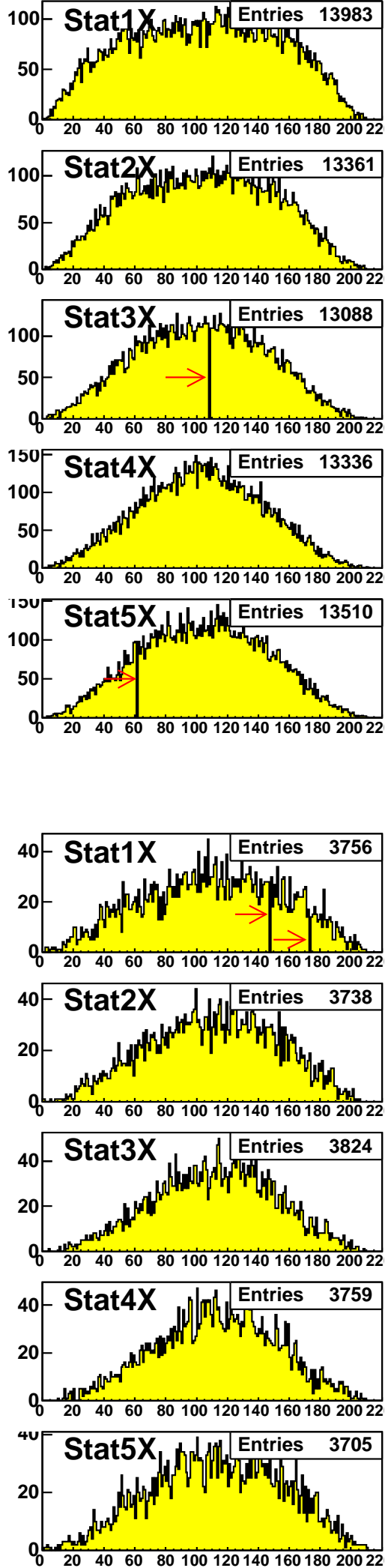
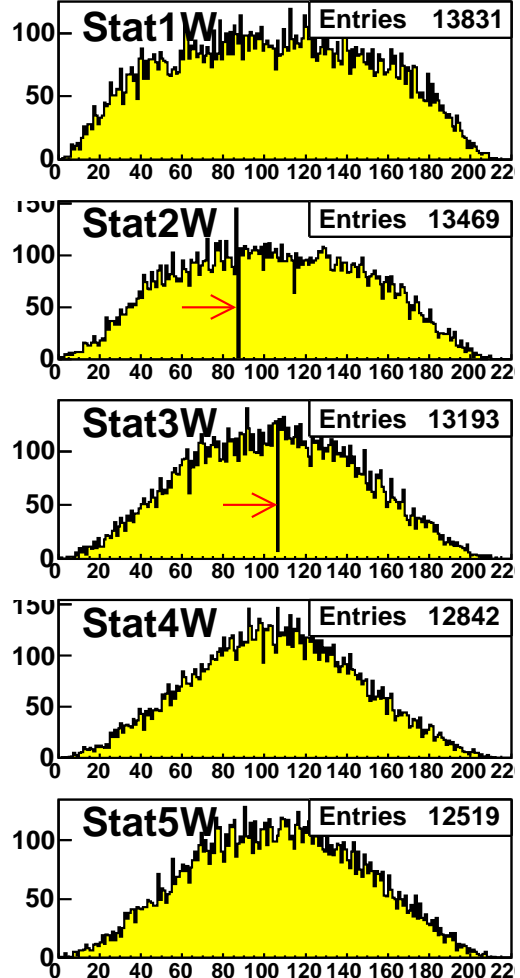
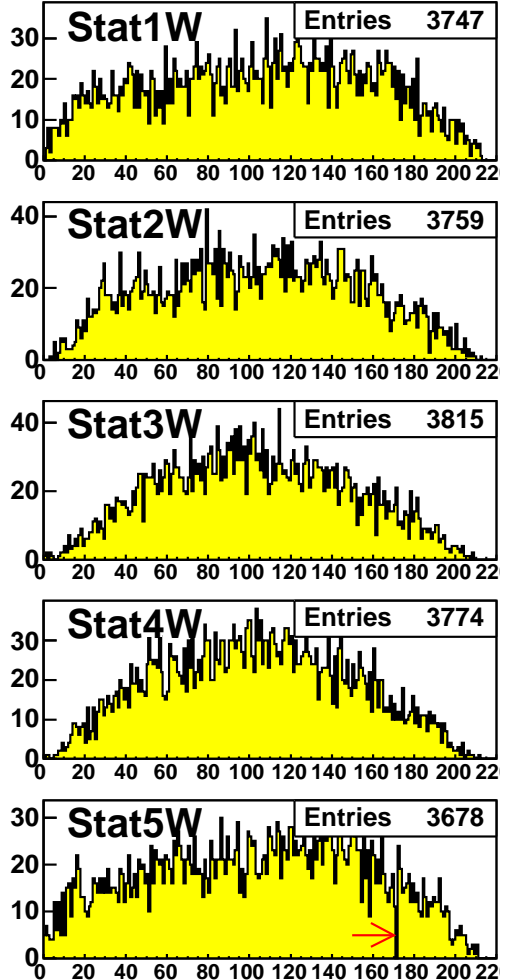

Figure 7.27: Hit fiber distributions for all the views measured at Tracker No.1 (top) and No.2

(bottom). Fibers connected with VLPC dead channels are indicated by the red arrows. 


\section{Tracker1}
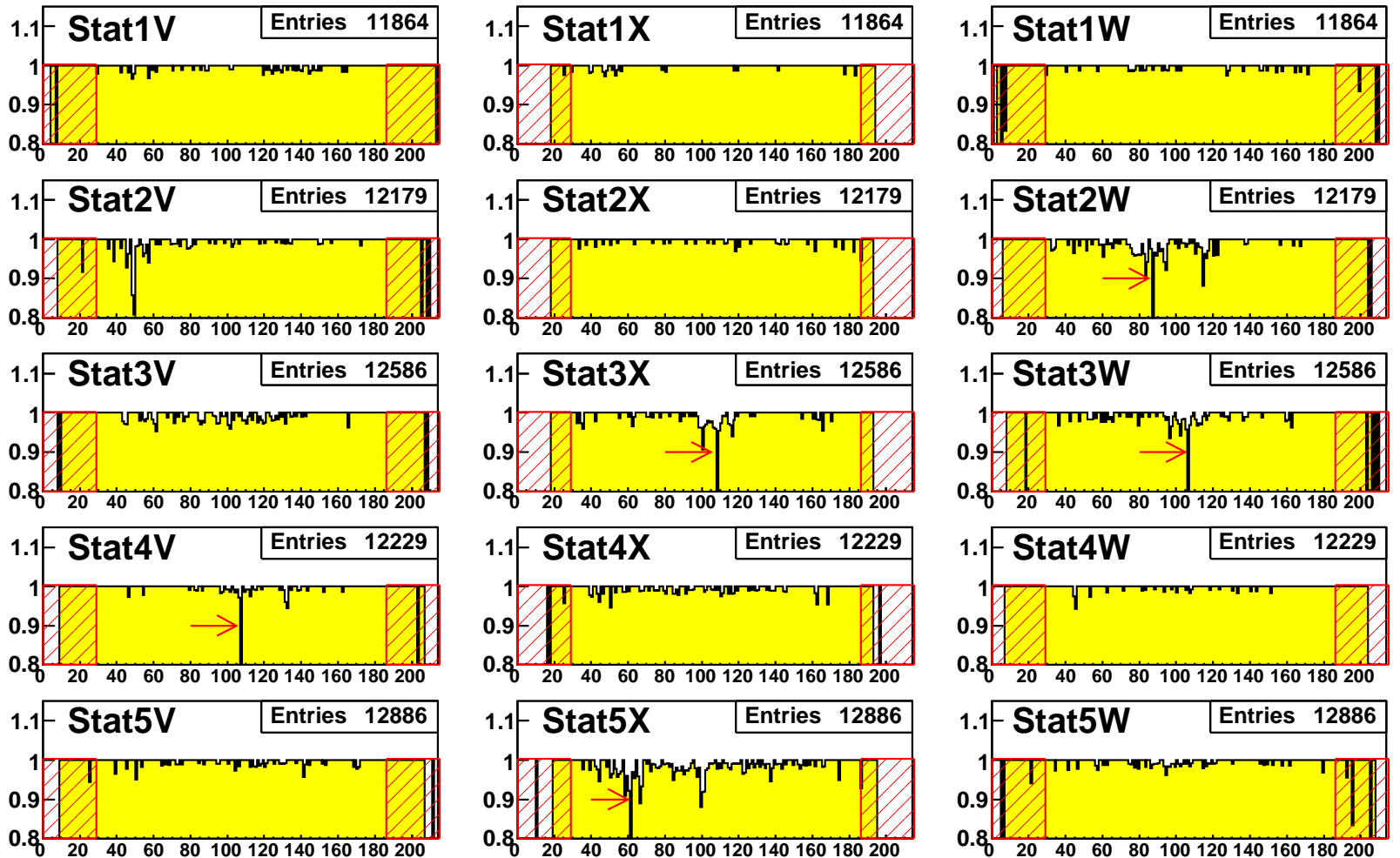

\section{Tracker2}
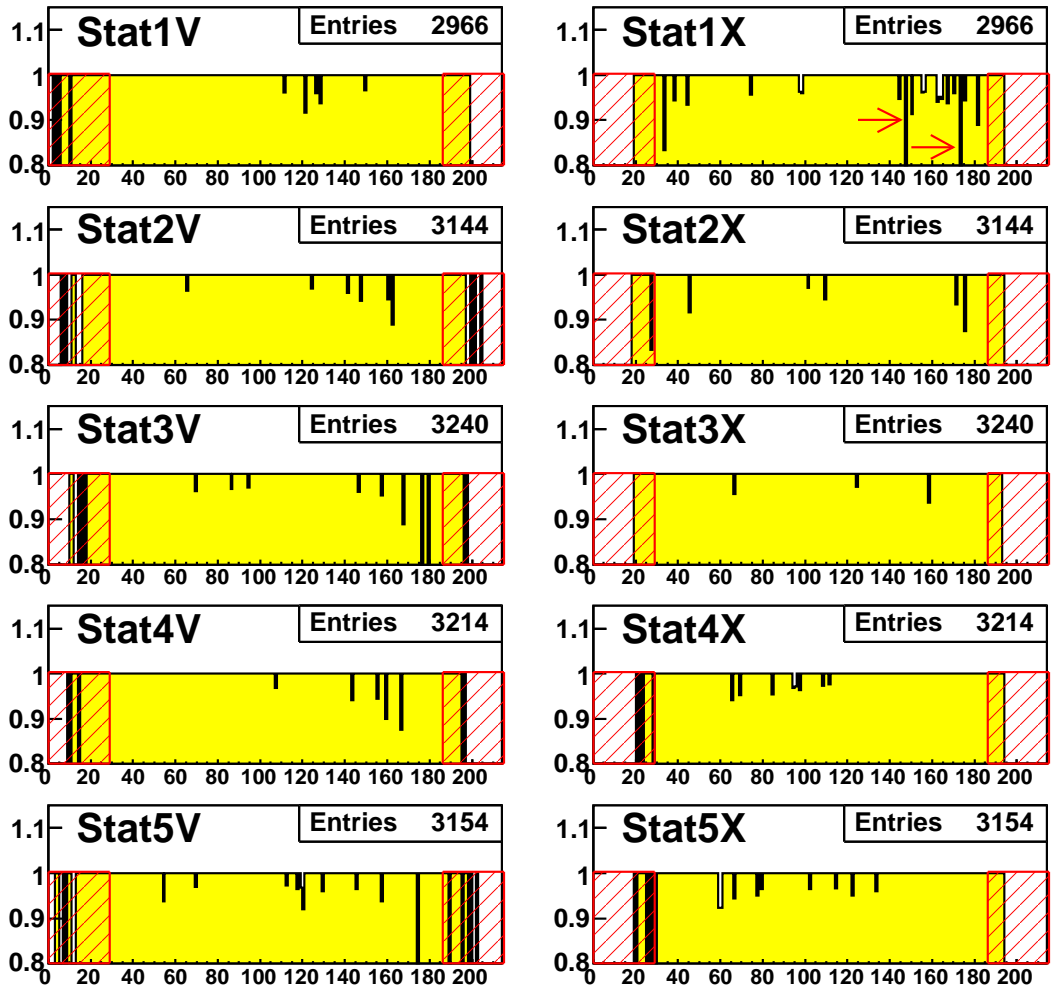
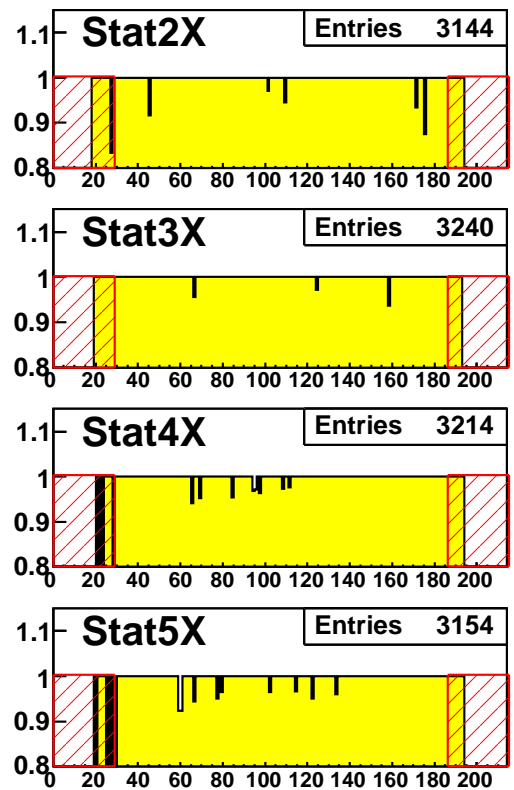
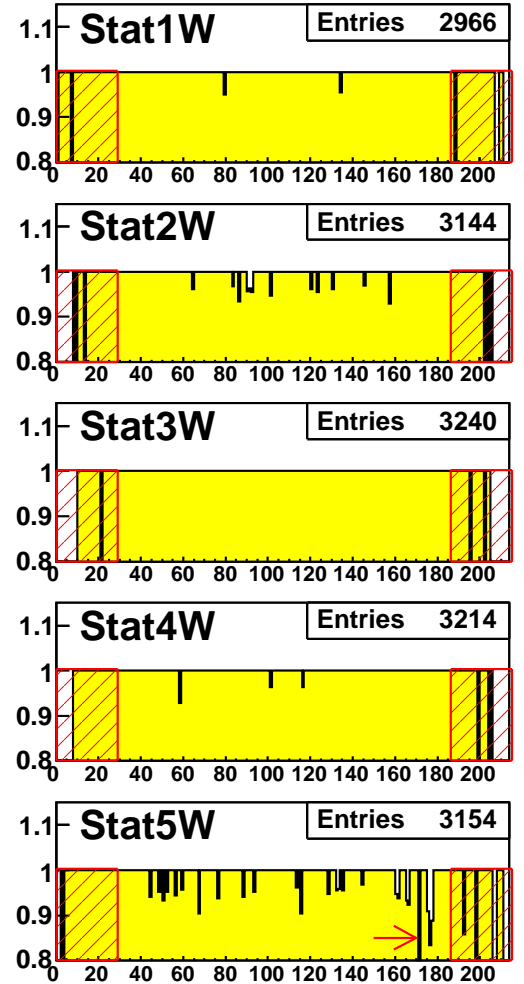

Figure 7.28: The distributions of hit efficiencies for all the views measured at Tracker No.1 (top) and No.2 (bottom). Fibers in hatched region are not included in the calculation of efficiency due to small statistics. Fibers connected with VLPC dead channels are indicated by the red arrows. 


\section{Tracker1}
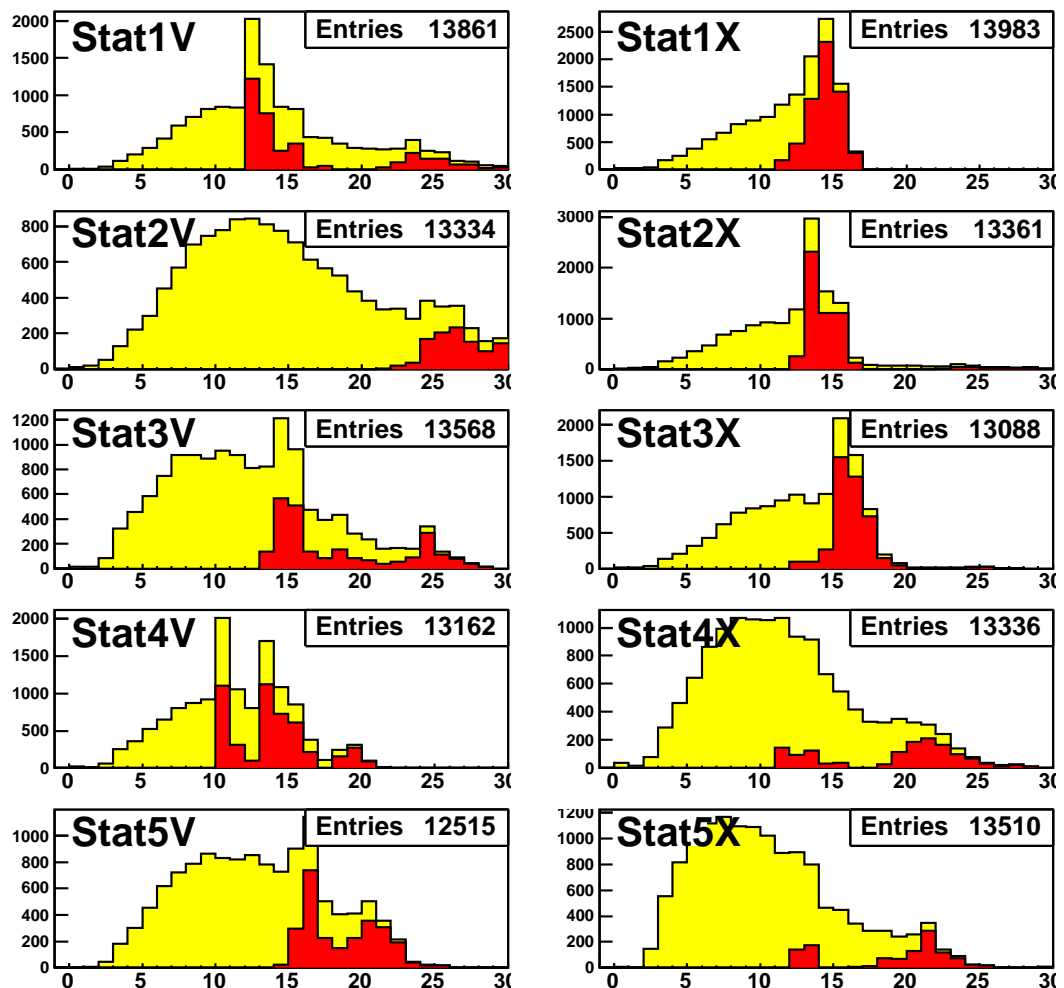

Tracker2
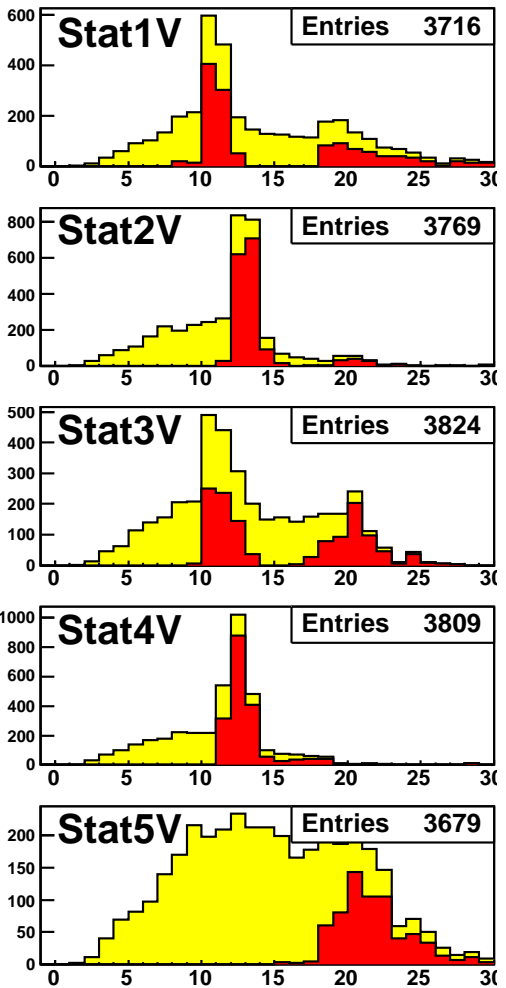
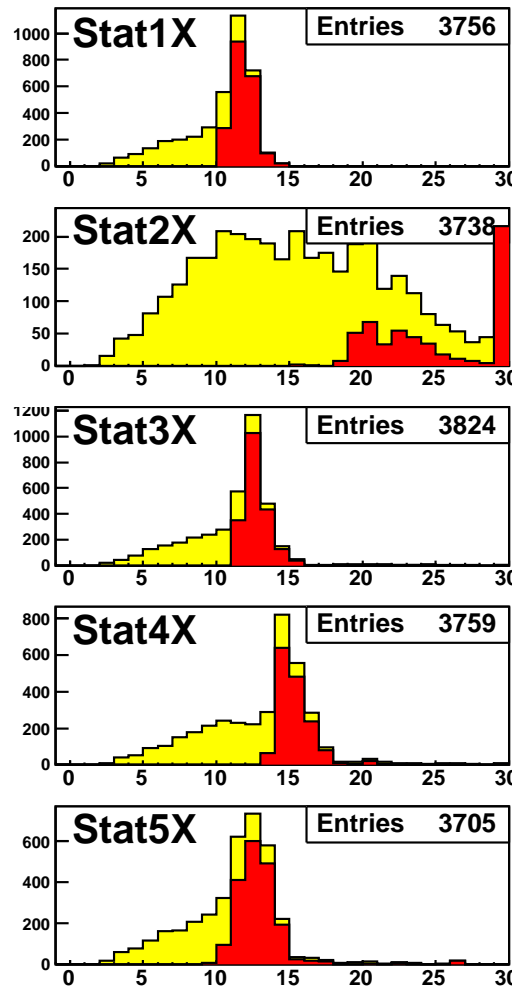
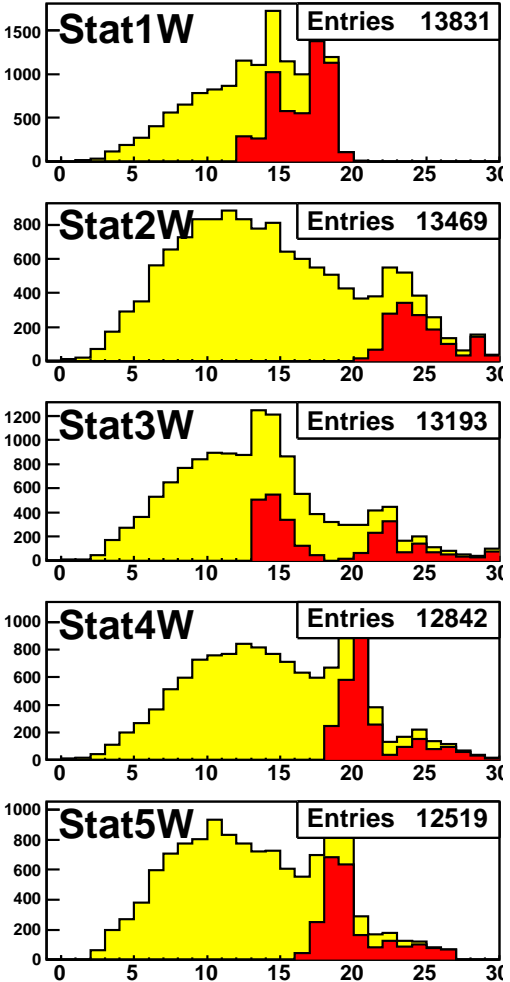
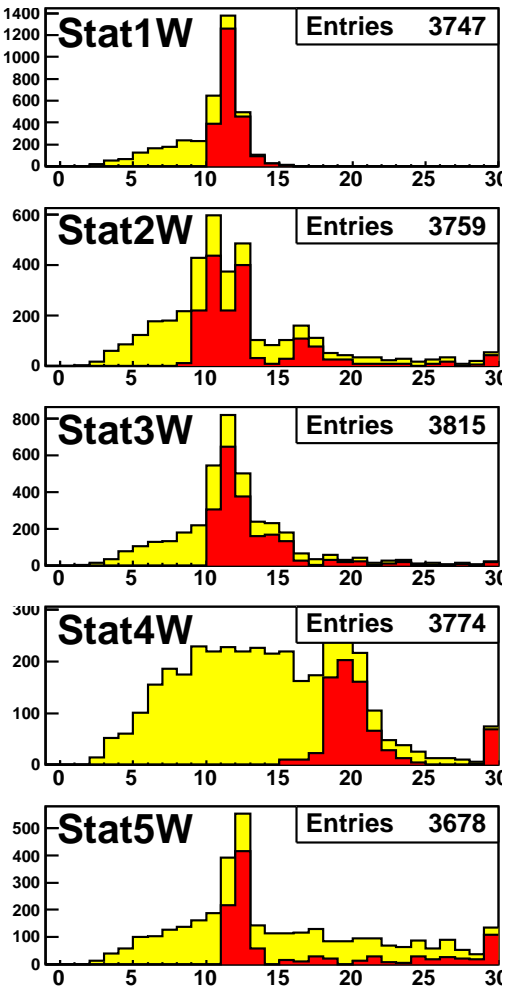

Figure 7.29: Light yields distributions of hit fibers for all the views measured at Tracker No.1 (top) and No.2 (bottom). The red histograms correspond to the ADC-saturated events. 


\section{Tracker1}
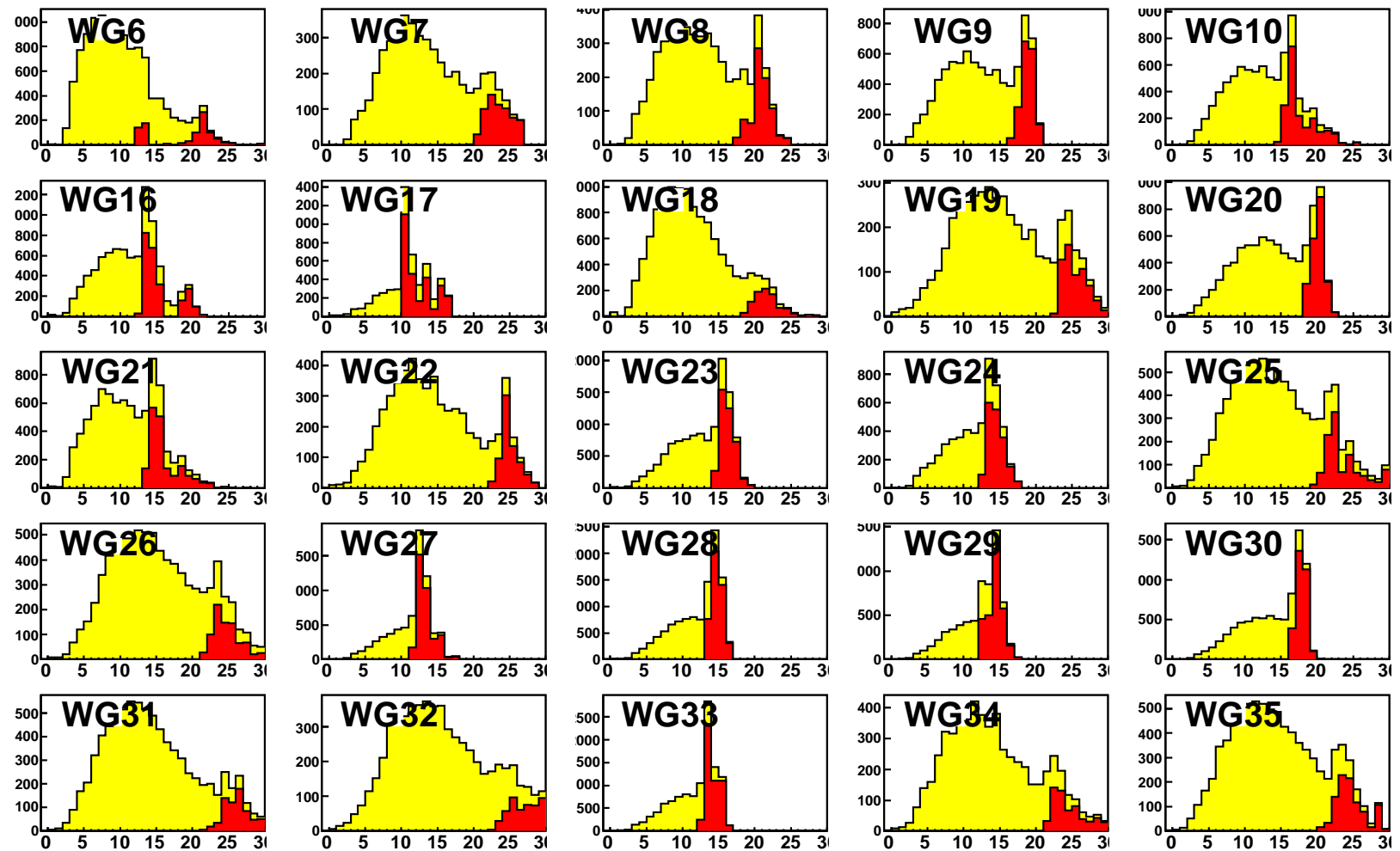

\section{Tracker2}
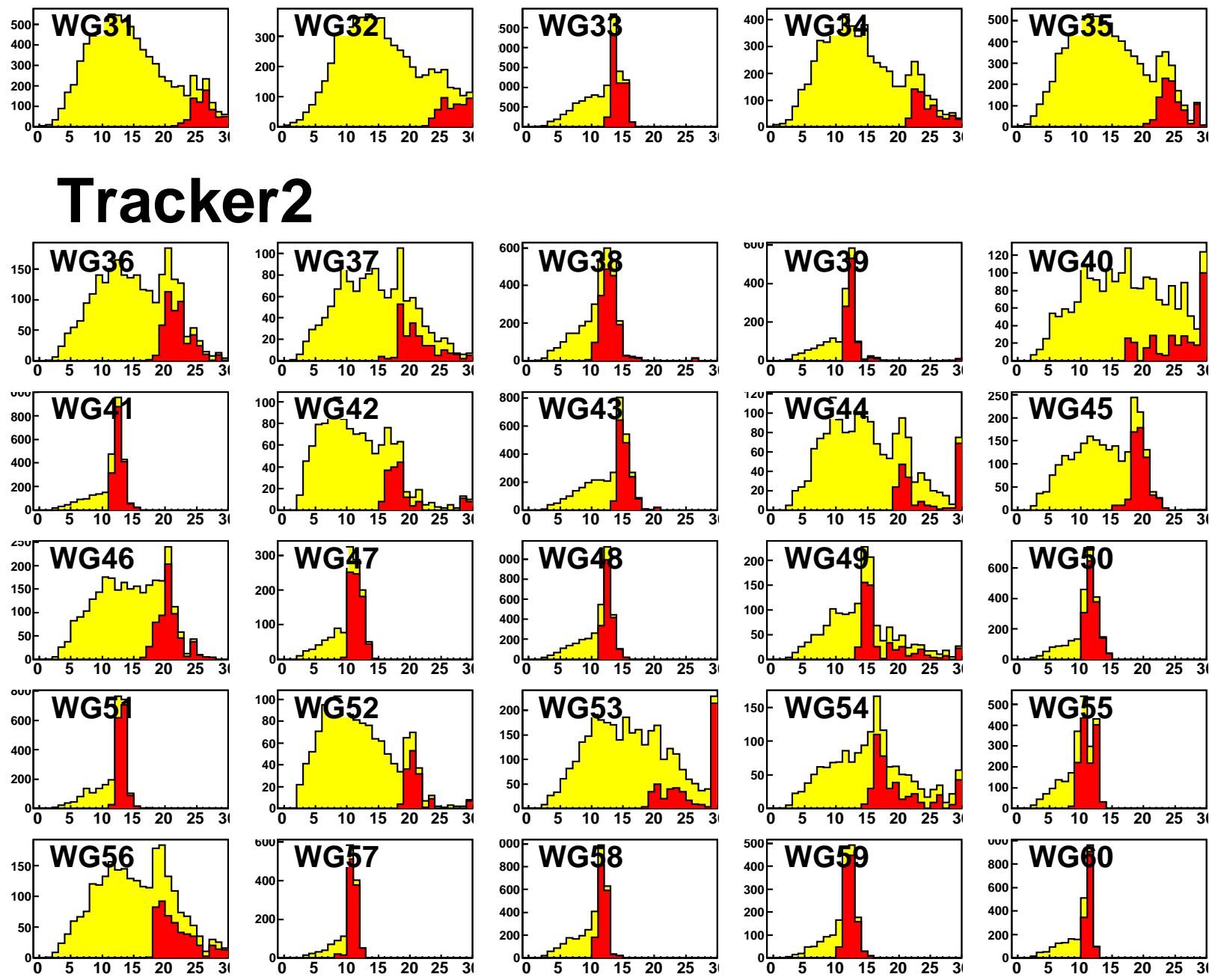

Figure 7.30: Light-yield distributions of hit fibers in terms of the light guide numbers used for Tracker No.1 (top) and No.2 (bottom). Red histograms correspond to the ADC-saturated events. 

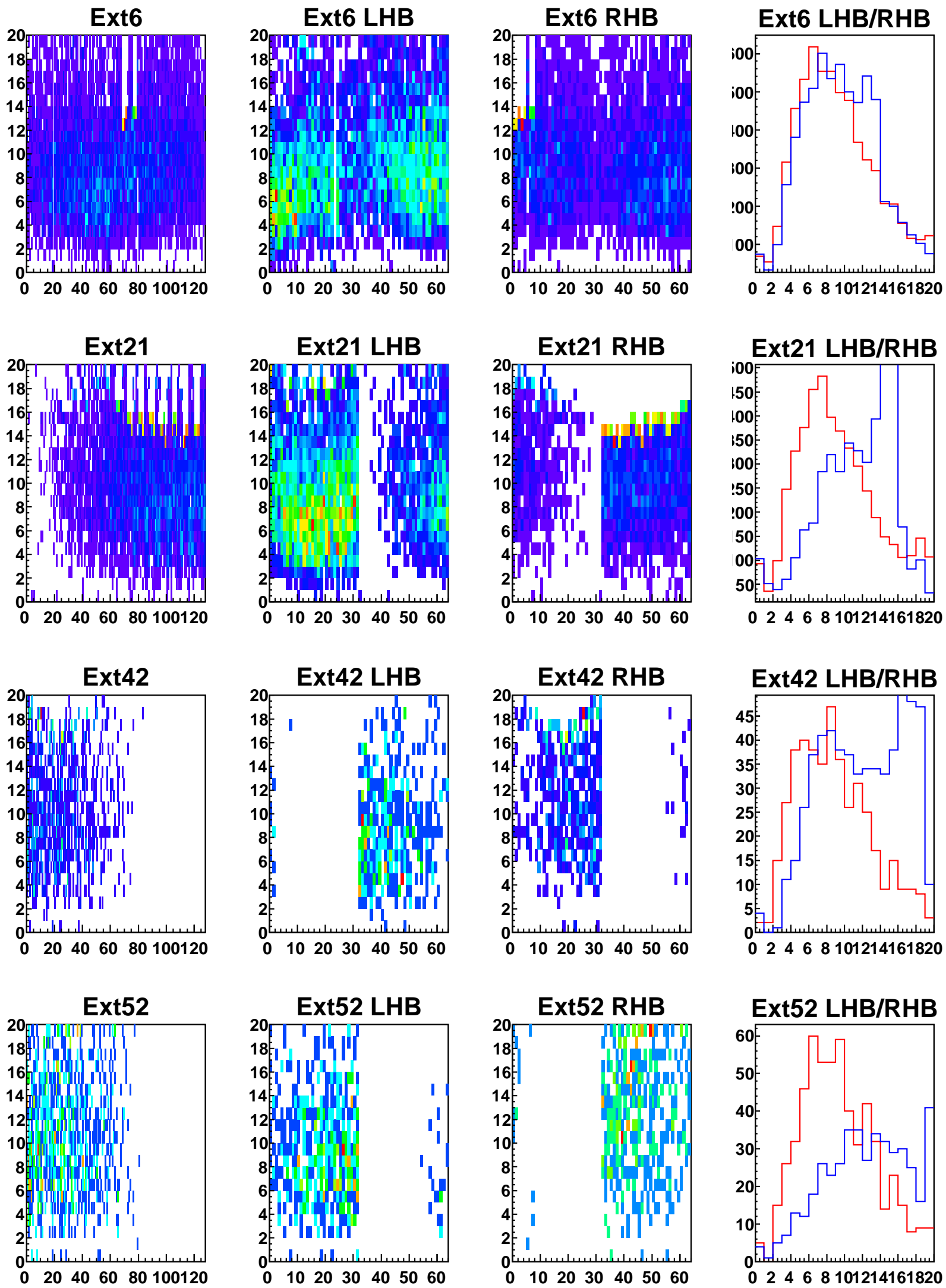

Figure 7.31: Light-yield distributions of hit fibers in terms of the light guide numbers used for Tracker No.1 (top) and No.2 (bottom). Red histograms correspond to the ADC-saturated events. 


\begin{tabular}{cccc}
\hline Light-guide number & Tracker & Station & View \\
\hline 6 & 1 & 5 & $\mathrm{X}$ \\
21 & 1 & 3 & $\mathrm{~V}$ \\
42 & 2 & 4 & $\mathrm{~V}$ \\
52 & 2 & 2 & $\mathrm{~V}$ \\
\hline
\end{tabular}

Table 7.6: Connection of light-guide number to view where small light-yields are found. 


\section{Chapter 8}

\section{Discussion}

\subsection{Effects on misalignments}

Effects on emittance measurements due to misalignments are studied using muon beams of average energy $200 \mathrm{MeV} / c$. Initial emittance of about $5 \mathrm{~mm}$-pi are generated by passing through a lead plate of $15 \mathrm{~mm}$ thickness. Emittance is calculated by reconstructing $100 \mathrm{k}$ muons in the method described in the section 5.4.4.

Firstly, effects are investigated with trackers that have the same amount of misalignments found at the Tracker No.1 or No.2. Differences of reconstructed emittance with and without applying these misalignments are calculated. As the result, the differences becomes about $1 \%(0.6 \%)$ at the Tracker No.1 (No.2), which exceeds the requirement of the emittance measurement. Therefore, the alignment calibrations described in chapter 7 are important for measuring emittance with precisions required at the MICE.

Finally, effects of small misalignments that could be remained after the correction are examined. As misalignment, three kinds of components are considered as as follows.

1. Translation by $50 \mu \mathrm{m}$ in $\mathrm{x}$-axis

2. Rotation by 0.1 degrees in $x-y$ space

3. Angle of z-axis between a tracker and a magnetic-filed by 0.05 degrees

Using these components, 16 patterns are examined as follows.

\begin{tabular}{|c|c|c|c|c|c|c|c|c|c|c|c|c|c|c|c|c|c|}
\hline Item & 0 & 1 & 2 & 3 & 4 & 5 & 6 & 7 & 8 & 9 & 10 & 11 & 12 & 13 & 14 & 15 & 16 \\
\hline \hline Station 1 & & $\mathrm{T}$ & & & & & $\mathrm{T}$ & $\mathrm{T}$ & $\mathrm{A}$ & & & & & $\mathrm{A}$ & $\mathrm{A}$ & $\mathrm{Z}$ & $\mathrm{Z}$ \\
\hline Station 2 & & & $\mathrm{T}$ & & & & & & & $\mathrm{A}$ & & & & & & & \\
\hline Station 3 & & & $\mathrm{T}$ & & & & & & & $\mathrm{A}$ & & & & & & \\
\hline Station 4 & & & & $\mathrm{T}$ & & & & & & & $\mathrm{A}$ & & & & & \\
\hline Station 5 & & & & & & $\mathrm{T}$ & $\mathrm{T}$ & $-\mathrm{T}$ & & & & & $\mathrm{A}$ & $\mathrm{A}$ & $-\mathrm{A}$ & $\mathrm{Z}$ & $-\mathrm{Z}$ \\
\hline
\end{tabular}

Table 8.1: Possible misalignmets after the correction. T(-T) denotes a translation of a station by $50(-50) \mu \mathrm{m}$ in the x-axis. A(-A) denotes an angular misalignment of a station by $0.1^{\circ}\left(-0.1^{\circ}\right)$ in the $\mathrm{x}-\mathrm{y}$ space. $\mathrm{Z}(-\mathrm{Z})$ denotes an angular misalignment of the $\mathrm{z}$-axis between a tracker and a magnetic-field by $0.05^{\circ}$. 
Emittance shifts found at each items are shown in Figure 8.1. For all of the items, effects on the emittance measurements are less than $0.05 \%$.

Mean and RMS of position and momentum residuals at each items are shown in Figure 8.2. The mis-alignments of a tracker z-axis tilting by 0.1 degrees (15 and 16) could be observable as shown in Figure 8.1, therefore this effects can be reduced by adjusting tracker axis in software.

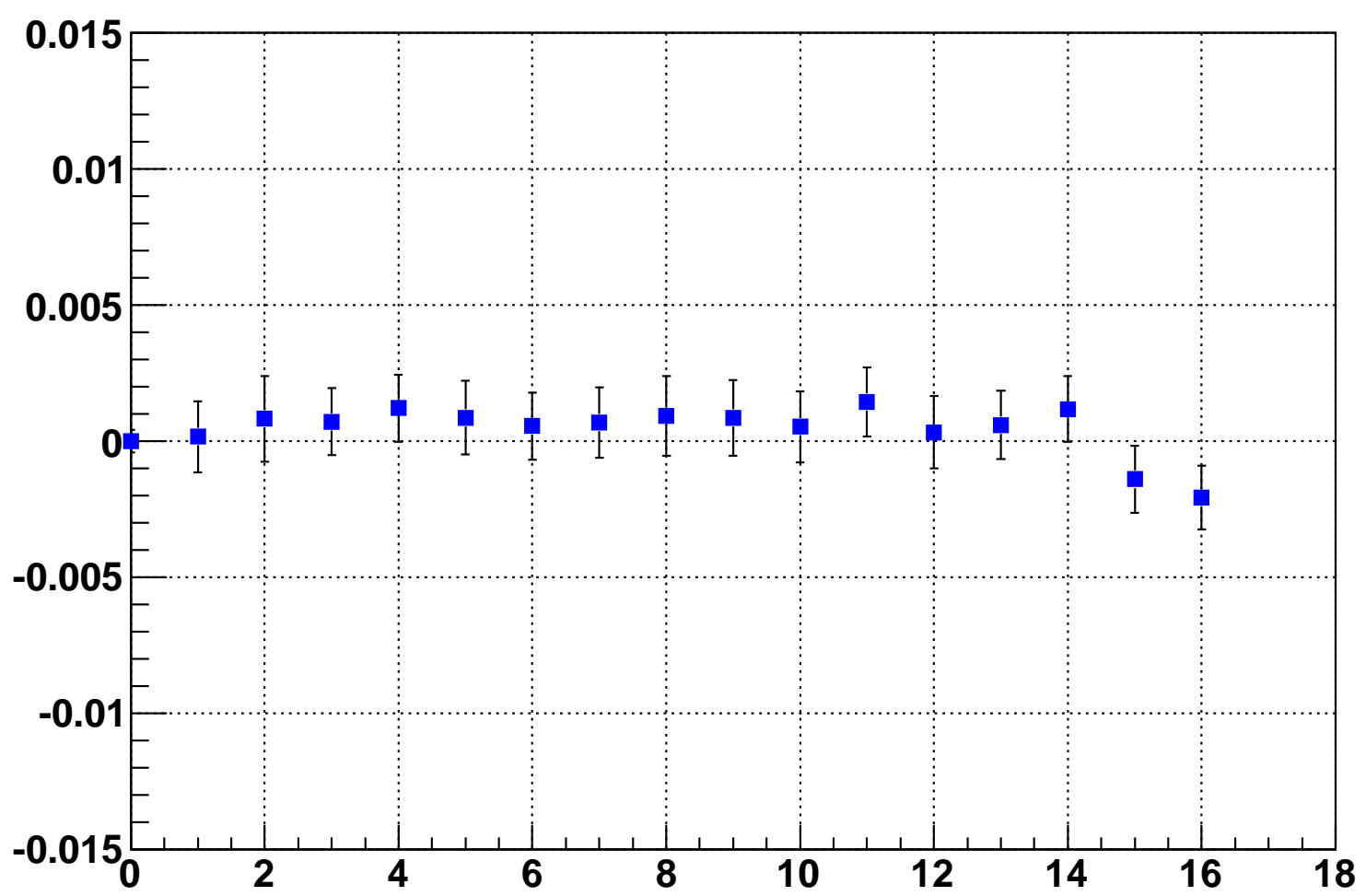

Figure 8.1: Emittance differences examined at several sets up of misalignments using four of $100 \mathrm{k}$ muons samples. Horizontal axis shows item numbers indicating different kinds of misalignments. Item number 0 indicates the result of perfectly aligned tracker. Descriptions of the item numbers are explained in Table 8.1. Vertical axis shows the ratio of the emittance changes at each case to that perfectly aligned case. Emittance changes are expected to be less than $0.5 \%$ for the possible misalignments

\subsection{Status and schedule of MICE SciFi Tracker}

As of January 2010, MICE SciFi tracker No.1 and No.2 are located at the Lab7 in the RAL to be installed to the spectrometer magnets. The magnets are being tested for their cooling performances which is expected to take few months (Figure 8.4). Until the time of the installation, two trackers will be tested to integrate DAQ for simultaneous readout of tracker No.1 and No.2. 
Mean
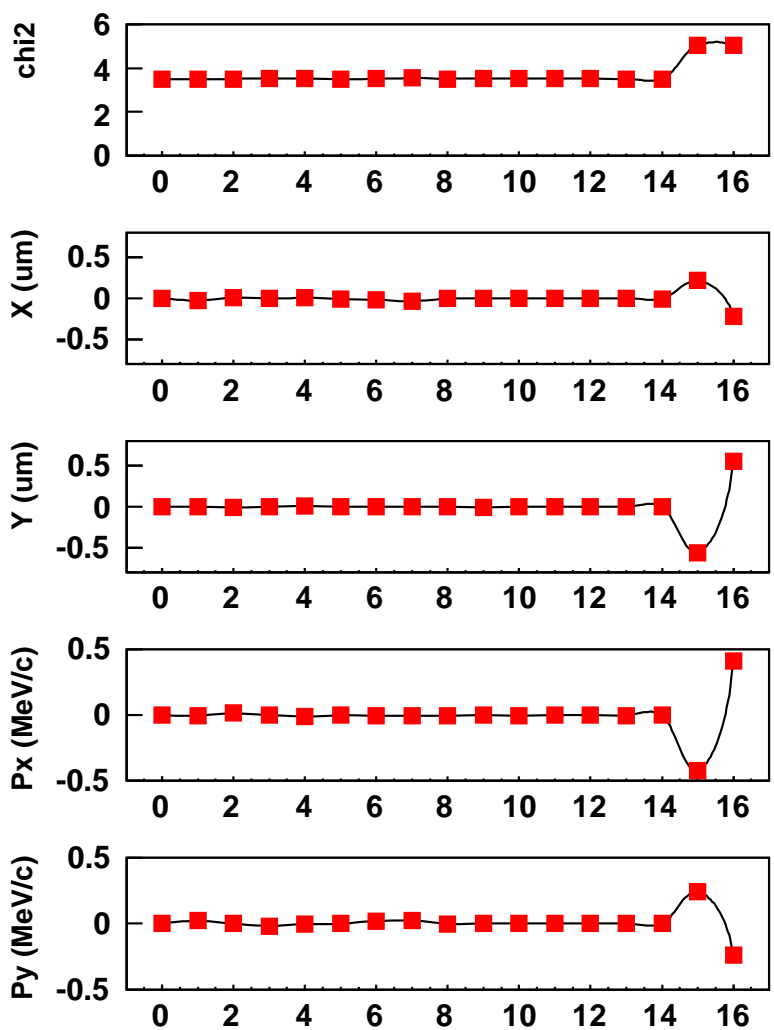

RMS
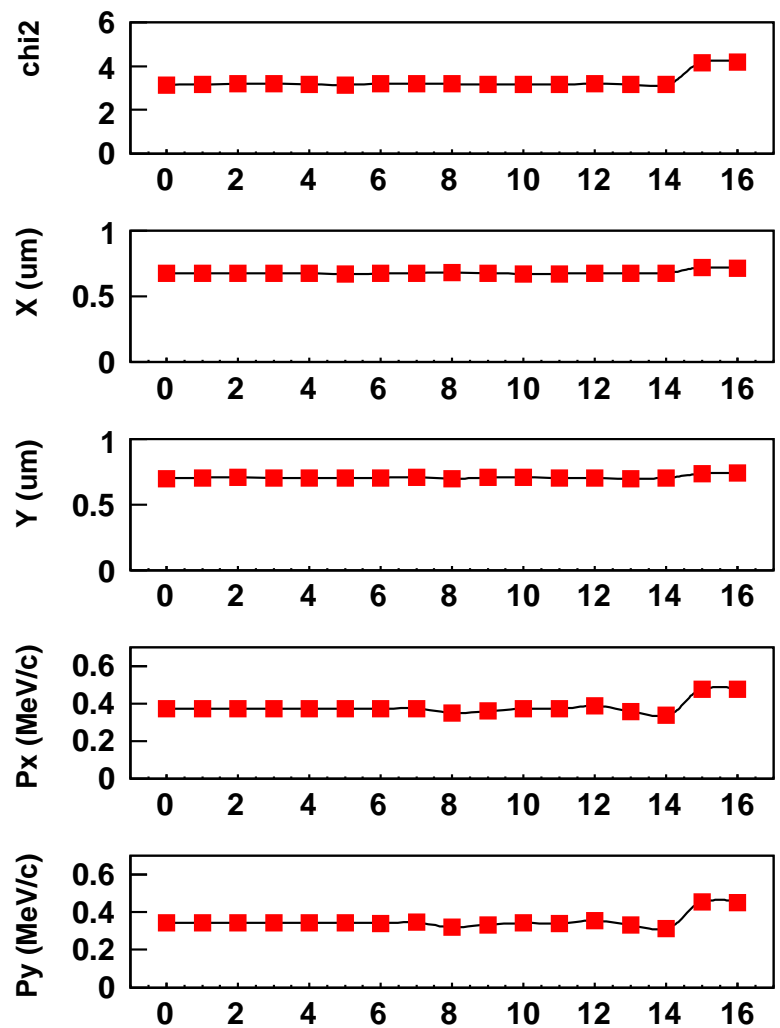

Figure 8.2: Plots showing the effects of misalignments with respect to chi-square (top), $\mathrm{x}, \mathrm{y}$ positions (second, third rows), and momentums (fourth, fifth rows). Left columns shows the mean and right columns shows the RMS of each components.

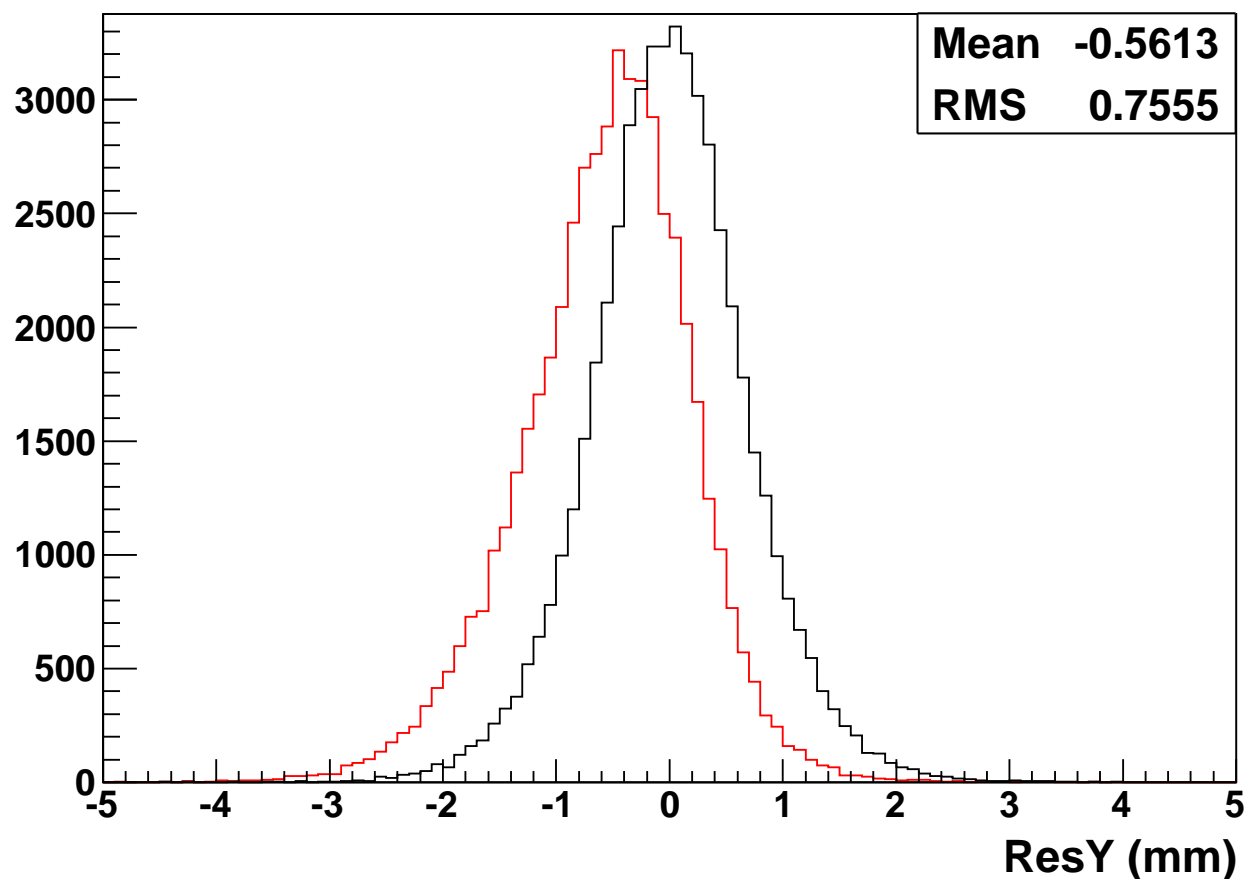

Figure 8.3: The Y residuals for misaligned of magnet axis by 0.1 degrees with respect to the tracker axis (red histogram, correspond to the item No.15) is superimposed on that with good aligned case (black histogram). 


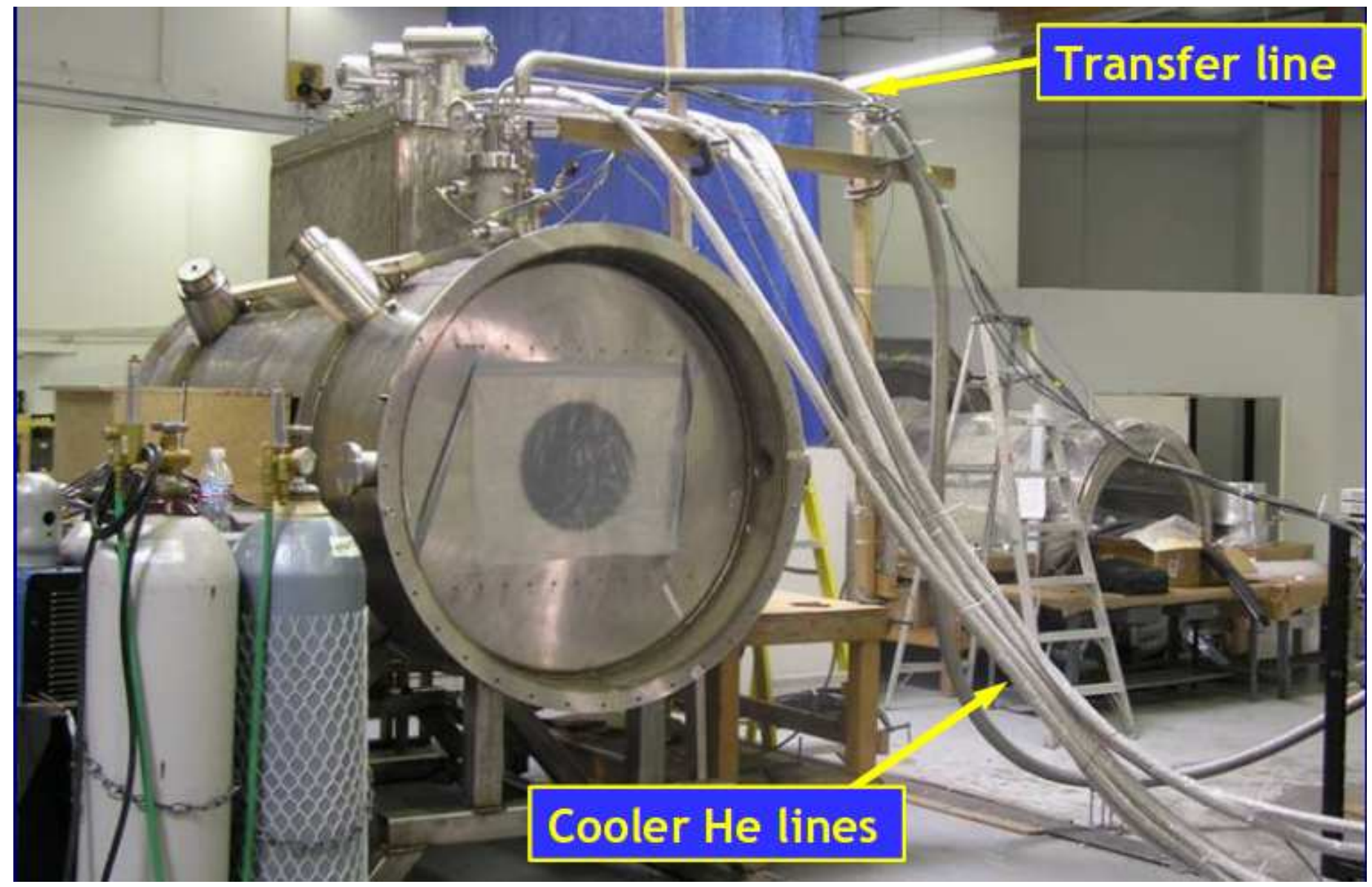

Figure 8.4: Photograph showing that spectrometer magnets attached with cooling He tubes for testing at the LBNL. 


\section{Chapter 9}

\section{Conclusion}

Scintillating fiber tracker based on $350 \mu \mathrm{m}$ diameter scintillating fibers have been developed for use in MICE. This is the very challenging task and some techniques are needed to realize since there are no trackers made with such a small diameter of scintillating fibers in the world. Upstream and downstream SciFi trackers have been successfully constructed with the international collaboration of UK, US and Japan by 2008. Both of the trackers have been tested with cosmic-rays at the RAL by 2009, at which high tracking efficiencies more than $90 \%$ are measured for both trackers. It is also confirmed that by collecting the misalignments found in both trackers, the requirement on the emittance measurement is achieved. 


\section{Acknowledgement}

I would like to thank Prof. Y. Kuno for giving me constructive comments and advises to finish the thesis. I have greatly benefied from Doc. M. Yoshida for continuous helps for works for a long time from the start of the MICE. Much of analysis can be proceeded through a plenty of discussion to him. I want thank Prof. K. Long and Prof. A. Bross for supporting and helping me for various works such as the construction and cosmic-ray test of the trackers during the stay at Imperial College, the RAL and the Fermilab. I also thank to Doc. M. Ellis, A. Fish, K. Walaron and C.Macwaters whose help me for working on the cosmic-ray test at the RAL and FNAL and KEK beam test. I would like to thank to P. Rubinov, K. Bowie and T. Hart for giving me great advices about the AFEII and VLSB boards to finish the implementation of the DAQ. I thank to Doc. T. Matsushita and for giving great helps on works such as station QA during the stay in IC. I want to thank Doc. J. Leaver for helping the test of slow control at the cosmic-ray test for Tracker 1 and works on the beam monitor for the MICE beam line. Great helps on the construction of the tracker are given from G. Barbar and Roger and ladies in IC. We cannot construct trackers successfully without their helps. I thank to M. Goto and ladies in G-tech for making light-guides from more than 10k of clear fibers with very good qualities. The KEK beam test can be achieved by the strong support from A. Horikoshi, K. Sakai K. Yamada and A. Sato, M. Aoki and K. Yoshimura in KEK. I would like to thank to S. Ishimoto, specialist of cryogenics during the stay in the FNAL and collaboration meetings. Many technical advises on fibers was given from A. Arita and O. Shinji in Kuraray CO., LTD.. Finally, I am appreciate for Prof. Y. Kuno for giving me a chance to take part in the MICE from the first period of the experiment. 


\section{Bibliography}

[1] Proposal to the Rutherford Appleton Laboratory, An International Muon Ionization Cooling Experiment, 2003.

[2] While 44 or $88-\mathrm{MHz}$ cavities have certain advantages, compared to 201-MHz cavities they occupy a lager volume, provide less gradient, and require more power. Moreover, while 201$\mathrm{MHz}$ power-supply components are available from CERN, 44- or 88- $\mathrm{MHz}$ supplies would need to be newly purchased or fabricated.

[3] M. Adiolfi et al.(KLOE Collaboration), Nucl.Instrum.Meth.A 478 (2002) 138

[4] M.Adinolifi et al., Nucl.Instrum.Meth.A482 (2002) 364

[5] Alan D. Bross and Anna Pla-Dalmau, 'Radiation Effects in Intrinsic 3HF Scintillator', Fermi National Accelerator Laboratory

[6] C.Albright et al., PysicsataNeutrinoFactory, Fermilab-FN-692, May 10, 2000, http://arXiv.org/ps/hep-ex/0008064

[7] M.Aleksa et al, Beam Dynamics Study of a Muon Ionization Cooling Experiment, Neutrino Factory Note 108 (2002), http://nicewww.cern.ch/ molat/neutrino/nf108.pdf

[8] C.Altunbas et al., Nucl.Instrum.Meth.A 490 (2002) 177

[9] C.Ankenbrandt et al., Phys.Rev.ST Accel.Beams 2, 081001 (1999)

[10] Antonelli et al., Nucl.Instrum.Meth.A354 (1995) 352

[11] M.Apollonio et al, OscillationPhysicswithaNeutrinoFactory, hep-ph/0210192 October 2002 (ed.M.Campanelli) http://arXiv.org/ps/hep-ph/0210192

[12] P.Aprili et al., ICARUS Coll., CERN-SPSC-2002-027, CERN-SPSC-P-323, Aug 2002

[13] ASME Boiler and Pressure Vessel Code, ANSI/ASME BPV-VIII-1 (American Society of Mechanical Enginners, New York, 1980), part UG-32.

[14] http://europa.eu.int/comm/enterprise/atex/

[15] B.Autin, A.Blondel and J. Ellis eds, CERN yellow report CERN-99-02, ECFA 99-197

[16] J.Aysto et al: Physcis with Low-Energy Muons at a Neutrino Factory Complex, hep$\mathrm{ph} / 0109217$

[17] S.Bachmann et al., Nucl.Instrum.Meth. A 478 (2002) 104

[18] V. Balbekov et al., The Single Field Flip Cooling Channel for a Neutrino Factory, Note 125 (2000) and The Double Flip Cooling Channel (Study II version), MUCOOL 203 (2001), both available from http://www-mucool.fnal.gov/notes/notes.html

[19] R. D. Ball, D. A. Harris, K. S. McFarland, hep-ph/0009223.

[20] V. Barger, S. Geer, R. Raja, K. Whisnant, Phys. Rev. D63 (2001) 033002.

[21] D. Bartlett et al., Nucl. Instrum. Meth. A260 (1987) 55.

[22] V. D. Elvira, P. Lebrun, P. Spentzouris, http://www-cpd.fnal.gov/geant4/G4BeamTools/

[23] BESS Collaboration, Nucl. Instrum. Meth. A455 (2000) 596; S. Geier et al., Proceedings of the 26th ICRC, Salt Lake City, 1999, 3, 117.

[24] A. Blondel et al., hep-ph-0105297. 
[25] S. J. Blundell, Contemp. Phys. 40, 175 (1999).

[26] A. Bressan et al., Nucl. Instrum. Meth. A 423 (1998) 119

[27] G.I. Budker, in Proc. of the 7th Int. Conf. on High Energy Accelerators, Yerevan, 1969.

[28] J. Burguet-Castell et al., hep-ph/0207080.

[29] G. Catanesi, Hit reconstruction, pattern identification and track measurements in the MICETPG, http://gabri.home.cern.ch/gabri/MICE/

[30] L. Chevalier, B0 Magnetic field measurement, Saclay Muon Software Group, internal report

[31] J. Corlett et al., in Proceedings of the 2001 Particle Accelerator Conference, Chicago, Editors P. Lucas, S. Webber (IEEE, Piscataway, NJ, 2001), p 500

[32] S. F. J. Cox, J. Phys. C20, 3187 (1987).

[33] M. A. Cummings et al., Current LH2-Absorber R\&D in MUCOOL, to appear in Proc. Nufact '02 Workshop.

[34] S. Abachi et al. The D0 Upgrade, Nucl. Instrum. and Meth., A408, pg. 103-109,1998; A. Bross et. al., Characterization and Performance of Visible Light Photon Counters (VLPCS) For The Upgraded D0 Detectgor at The FERMILAB Tevatron, Nucl. Instrum. and Meth., A477, pg. 172-178,2002.

[35] P. Dalmas de Reotier and A. Yaouanc, J. Phys. C9, 9113 (1997).

[36] A. Donini, D. Meloni and P. Migliozzi, hep-ph/0206034.

[37] C. Wyss et al., http://muonstoragerings.web.cern.ch/muonstoragerings/emcog/workingplan.doc

[38] S. Farinon and P. Fabbricatore, First Thoughts about the Design of the Spectrometer Solenoids for the MICE Experiment, a Power Point presentation by S. Farinon at the MICE workshop at LBNL, 23 to 25 October 2002

[39] R. Fernow, ICOOL, A Simulation Code for Ionization Cooling of Muons Beams, Proc. 1999 Particle Accelerator Conference, A. Lucio and W. MacKay, eds. (IEEE, Piscataway, NJ, 1999), p. 3020.

[40] G. Franchetti et al., Muon phase rotation and cooling: simultion work at CERN, CERN/PS 2002-076(AP) CERN NUFACT Note 119 (2002) to appear in the proceedings of NUFACT02.

[41] Y. Fukuda et al. (SuperKamiokande Collab.), Phys. Lett. B433, 9 (1998); Phys. Lett. B436, 33 (1998); Phys. Rev. Lett. 81, 1562 (1998); Phys. Rev. Lett. 82, 2644 (1999)

[42] U. Gastaldi, TPG, a cylindrical imaging gas detector with high transparency in the axial direction, INFN-LNL MICE-Legnaro Note01/02 May 2002, http://proj-bdlnice.web.cern.ch/projbdl-nice/cool/gastalditpgdraft.pdf and http://hep04.phys.iit.edu/cooldemo/gastaldi32002.pdf

[43] The CERN Gas Detector Development group page: http://gdd.web.cern.ch/GDD

[44] The Geant4 Tool Kit is available at http://wwwinfo.cern.ch/asd/geant4/geant4.html

[45] S. Geer, PRD 57, 6989 (1998)

[46] P. Gorodetzky et al., HELLAZ Coll., Nucl.Instrum.Meth.A471:131-135,2000.

[47] M. A. Green, J. Y. Chen and S. T. Wang, The Design, and Construction of a Gradient Solenoid for the High Power RF Cavity Experiment for the Muon Collider, Institute of Physics Conference Series 167, p 1199, (2000),

[48] M. A. Green and J. M. Rey, Superconducting Solenoids for an International Muon Cooling Experiment, to be published in IEEE Transactions on Applied Superconductivity 13, No. 2

[49] M. A. Green, The Cryogenic Refrigeration System for MICE, LBNL Report LBNL-51751, November 2002

[50] The Study of a European Neutrino Factory Complex, P. Gruber ed., Neutrino Factory Note 103(2002) CERN/PS/2002-080(PP) http://tilde-pgruber.home.cern.ch/ pgruber/docs/nf_report/nf_report_draft4.doc 
[51] M. Guler et al., OPERA Coll., CERN-SPSC-2001-025, CERN-SPSC-M-668, LNGS-EXP-302001-ADD-1, Aug 2001.

[52] K. Hanke, Muon Front-End without Cooling, NuFact Note 59 (2000).

[53] http://harp.web.cern.ch/harp/Classified/Sub_detectors/TPC/index.html

[54] SYLP0 by Quanta Systems srl, Milano, Italy

[55] G. Barichello et al., The HARP TOF Wall Construction and Test, INFN/AE-02/02.

[56] E. B. Holzer: Figure of merit for muon cooling, CERN Nufact Note 111 (2003)

[57] E. B. Holzer: Simulation of the $88 \mathrm{MHz}$ cooling experiment with ICOOL, CERN Nufact Note $124(2003)$

[58] P. Janot, Workshop on an International Muon Ionization Cooling Experiment, CERN, October 2001, http://muonstoragerings.cern.ch/October01WS/oct01ws.html

[59] A Feasibility Study of A Neutrino Factory in Japan, Y. Kuno, ed., http://wwwprism.kek.jp/nufactj/index.html

[60] Japan Proton Accelerator Research Complex J-PARC http://jkj.tokai.jaeri.go.jp/

[61] K. Eguchi et al., KamLAND Coll., First Results from KamLAND: Evidence for Reactor AntiNeutrino Disappearance, submitted to Phys. Rev. Lett. (6 Dec. 2002). 134

[62] D. M. Kaplan, Introduction to Muon Cooling, in Proc. APS/DPF/DPB Summer Study on the Future of Particle Physics (Snowmass 2001), N. Graf, ed. (2002), physics/0109061.

[63] D. M. Kaplan et al., Progress in Absorber R\&D for Muon Cooling, to appear in Proc. 3rd International Workshop on Neutrino Factory based on Muon Storage Rings (NuFACT'01), Tsukuba, Japan, 24?30 May 2001, arXiv:physics/0108027.

[64] D. M. Kaplan et al., Progress in Absorber R\&D 2: Windows, in Proc. 2001 Particle Accelerator Conference, P. Lucas and S. Webber, eds. (IEEE, Piscataway, NJ, 2001), p 3888 (arXiv:physics/0108028).

[65] The KLOE Collaboration, The KLOE Detector Technical Proposal, LNF-92/019 (1992)

[66] D. Kubik et al., to be submitted to Nucl. Instrum. Meth.

[67] K. Lang et al., MINOS Coll., Nucl. Instrum. Meth. A461, 290 (2001).

[68] P. M. Lapostolle, IEEE Trans. Nucl. Sci. NS-18, No 3, 1101 (1971)

[69] Acerbi et al., INFN/TC-92/23, October 1992, M. Bonesini et al., INFN/AE-94/14, May 1994

[70] M. Bonesini et al., Construction of a Fast Laser-based Calibration System for the HARP TOF Counters Wall, INFN/AE-02/02.

[71] T. Lasserre et al, LENS Coll., Prog. Part. Nucl. Phys. 48, 231 (2002).

[72] M. Lindner, The physics potential of future long baseline neutrino oscillation experiments, to appear in Neutrino Mass, Springer Tracts in Modern Physics, ed. G. Altarelli and K. Winter, hep-ph/0209083 (2002)

[73] A 40-80 MHz system for phase rotation and cooling, NUFACT Note 20 (2001), available from http://molat.home.cern.ch/molat/neutrino/nfnotes.html

[74] M. L. Mangano et al., CERN-TH/2001-131 (hep-ph/0105155).

[75] J. M. Meek and J. D. Craggs, Electrical Breakdown in Gases, John Wiley \& Sons, 1978, p. 557.

[76] MICE Letter of Intent to the Paul Scherrer Institute and Rutherford Appleton Laboratory, available from http://hep04.phys.iit.edu/cooldemo/micepsiloi.pdf

[77] Joint CLRC/PPARC Panel to Review the MICE Proposal (A. Astbury et al.), Report of The 1st Panel Meeting, 25 March 2002, available from http://hep04.phys.iit.edu/cooldemo/micenotes/public/doc/MICE0002/MICE0002.doc

[78] See the MICE web site http://hep04.phys.iit.edu/cooldemo 
[79] Mikhail, E. M., Bethel, J. S., and McGlone, J. C., 2001, Introduction to Modern Photogrammetry, New York, John Wiley \& Sons, Inc.

[80] MUCOOL expt. (Muon Ionization Cooling R\&D), FNAL Proposal P904 http://www.fnal.gov/projects/muon_collider/cool/cool.html

[81] H. Murayama, Int. J. Mod. Phys. A17, 3403 (2002).

[82] H. Edwards et al., http://www.cap.bnl.gov/mumu/polit/Mutac_Report_2001.pdf.

[83] NA49 Collaboration, Nucl. Instrum. Meth. A430 (1999) 210; Nucl. Instrum. Meth. A451 (2000) 406; NA52 Collaboration, Nucl. Phys. A 590 (1995) 347C; PAMELA Collaboration, 27th International Cosmic Rays Conference, OG

[84] D. Neuffer, Part. Acc. 14, 75 (1983);

[85] D. Neuffer, in Advanced Accelerator Concepts, F. E. Mills, ed., AIP Conf. Proc. 156 (American Institute of Physics, New York, 1987), p. 201; R. C. Fernow, J. C. Gallardo, Phys.Rev. E 52, 1039 (1995).

[86] D. Neuffer, mu+ mu- Colliders, CERN Yellow Report 99-12 (1999).

[87] J. Norem, A. Moretti and M. Popovic, Nucl. Instr. and Meth in Phys. Res. A 472 (2001) 600

[88] J. Norem, V. Wu, A. Moretti, M. Popovic, Z. Qian, L. Ducas, Y. Torun, and N. Solomey, Dark Current, Breakdown and B Field effects in a Multicell, $805 \mathrm{MHz}$ Cavity, Submitted to Phys. Rev. ST Accel. Beams, MUCOOL Note \#235.

[89] S. Geer, PRD 57, 6989 (1998); B. Autin, A. Blondel and J. Ellis eds, CERN yellow report CERN 99-02, ECFA 99-197; C. M. Ankenbrandt et al., Phys. Rev. ST Accel. Beams 2, 081001 (1999); A. Blondel et al., Nucl. Instrum. Methods Phys. Res., A 451 (2000) 102; C. Albright et al., FERMILAB-FN-692, hep-ex/0008064; D. Harris et al., Snowmass 2001 Summary, hepph/0111030; A. Cervera et al., Nucl. Phys. B579, 17 (2000), Erratumibid. B593:731-732,2001; M. Koike and J. Sato, Phys. Rev. D62 (2000) 073006.

[90] http://www-off-axis.fnal.gov/loi.ps, Letter of intent to build an off-axis detector to study $\mu_{\mu} \rightarrow$ $\mu_{e}$ oscillations with the NuMI neutrino beam, August 2002.

[91] See e.g. R. B. Palmer, Ring Coolers: Status and prospects, presented at NuFact '02 Workshop, London, England, 1-6 July 2002, available from http://www.hep.ph.ic.ac.uk/NuFact02/Scientificprogramme/files/Friday/plenary/A09_palmer.ps

[92] R. B. Palmer, and R. Fernow, $200 \mathrm{MHz}$ Cooling Experiment Design Version 5, MICE Note 18, November 20, 2002, available from http://hep04.phys.iit.edu/cooldemo/notes/notes.html.

[93] A. Perrin, PATH reference manual, unpublished.

[94] K. Hagiwara et al., Review of Particle Properties, Phys. Rev. D66, 010001 (2002), also available at http://pdg.lbl.gov

[95] Pressure Equipment Directive 97/23/EC.

[96] E. A. Perevedentsev, A. N. Skrinsky, Proc. 12th Int. Conf. on High Energy Accelerators, F. T. Cole, R. Donaldson, eds. (Fermilab, 1984), p. 485.

[97] A. Pilaftsis, Int. J. Mod. Phys. A14, (1999)

[98] Reference Manual for the POISSON/SUPERFISH Codes, report LA-UR-87-115.

[99] http://village.flashnet.it/users/polhitec/index.htm

[100] Richard E. Prael and Henry Lichtenstein, User Guide to LCS: The LAHET Code System, Los alamos National Laboratory report LA-UR-89-3014, Revised (September 15, 1989).

[101] Design of the PSI muon beam lines with solenoid decay channel are described in http://www.psi.ch

[102] E. Radicioni, TPG readout, http://proj-bdl-nice.web.cern.ch/proj-bdlnice/cool/tpgemiliomarch2002.pdf 
[103] RAL Health and Safety Notice No. 20, Protection Against Ionizing Radiation.

[104] RAL Health and Safety Notice No. 8, New Regulations on Pressure Systems and Transportable Gas Containers.

[105] RAL Health and Safety Notice No. 9, The Biological Effects of Magnetic Fields.

[106] Rutherford Appleton Laboratory safety Code No. 1, Hydrogen and Deuterium.

[107] Rutherford Appleton Laboratory safety Code No. 6, Precautions to Avoid Ignition of Flammable Gases by Static Electricity.

[108] The RIKEN-RAL pulsed muon facility, T. Matsuzaki, K. Ishida, K. Nagamine, I. Watanabe, G. H. Eaton, W. G. Williams, Nucl. Instrum. Meth. A465, 65 (2001).

[109] A. De Rujula, M.B. Gavela, P. Hernandez, Nucl. Phys. B547 (1999) 21, arXiv: hep$\mathrm{ph} / 9811390$.

[110] D. Sanders et al., IEEE Trans. Nucl. Sci. 49 (2002) 1834.

[111] F. Sauli, Nucl. Instrum. Meth. A461 (2001) 47

[112] F. Sauli, Nucl. Instrum. Meth. A 461 (1997) 531

[113] See http://www.shef.ac.uk/uni/academic/N-Q/phys/research/pa/SIREN.html.

[114] A. N. Skrinsky, V. V. Parkhomchuk, Sov. J. Part. Nucl. 12, 223 (1981); D. Neuffer, Part. Acc. 14, 75 (1983); E. A. Perevedentsev, A. N. Skrinsky, in Proc. 12th Int. Conf. on High Energy Accelerators, F. T. Cole, R. Donaldson, eds. (Fermilab, 1984), p. 485.

[115] Q. R. Ahmad et al., SNO Coll, Phys. Rev. Lett. 87, 071301 (2001)

[116] Q. R. Ahmad et al., SNO Coll., nucl-ex/0204008.

[117] Feasibility Study on a Neutrino Source Based on a Muon Storage Ring, D.Finley, N.Holtkamp, eds. (2000), http://www.fnal.gov/projects/muon_collider/reports.html

[118] Feasibility Study-II of a Muon-Based Neutrino Source, S. Ozaki, R. Palmer, M. Zisman, and J. Gallardo, eds. BNL-52623, June 2001, available at http://www.cap.bnl.gov/mumu/studyii/FS2-report.html.

[119] R. Suda et al. (Belle), Nucl. Instrum. Meth. A406 (1998) 213.

[120] http://www.lnf.infn.it/acceleratori/btf/

[121] The TRIUMF muon beam lines, http://musr.triumf.ca/equip/mubeamlines/node3.html

[122] M. S. Turner et al., Rev. Mod. Phys. 71, S145 (1999)

[123] W. T. Welford, R. Winston, The optics of non-imaging concentrators, Academic Press, New York, 1978, H. Hinterberger, R. Winston, Rev. Sci. Instr. 37 (1966) 1094

[124] Mini-Workshop on Beam Instrumentation, CERN, 13-14 July 2000; 2nd Workshop on Instrumentation for Muon Cooling Studies, IIT Chicago, Nov. 10-11, 2000; Workshop on Instrumentation for Muon Cooling Studies, Blackett Laboratory, Imperial College, 23-24 February 2001; Workshop on a Muon Ionization Cooling Experiment, 25-27 October 2001, CERN. Talks presented at these workshops can be accessed through the CERN muonstoragerings web page: http://muonstoragerings.cern.ch+ 